\title{
Biophysical Characterization of SNARE Complex Disassembly Catalyzed by NSF and $\alpha$ SNAP
}

\author{
Dissertation \\ zur Erlangung des Doktorgrades der \\ Mathematisch-Naturwissenschaftlichen Fakultäten \\ der Georg-August-Universität zu Göttingen
}

\author{
vorgelegt von \\ Ulrike Winter \\ aus Kiel
}

Göttingen 2008 
D 7

Referent: Prof. Dr. Reinhard Jahn

Koreferent: Prof. Dr. Ralf Ficner

Tag der mündlichen Prüfung: 


\section{Contents}

1 Introduction $\quad 1$

1.1 Function of the SNARE Proteins . . . . . . . . . . . . . . 2

1.2 A Closer Look at SNARE Assembly . . . . . . . . . . . . . . . . . . 2

1.2.1 The 'Zipper'-Model—How Does SNARE Assembly Proceed? . 2

1.2.2 The Driving Force - Spontaneous Formation of Extremely Stable Complexes . . . . . . . . . . . . . . . 3

1.2.3 The Logistics - SNAREs Switch from 'trans' to 'cis' During Membrane Fusion . . . . . . . . . . . . . . . . 4

1.3 Postfusion-Time for Recycling of SNARE Complexes . . . . . . . . . 6

1.3.1 NSF and the SNAPs - The Disassembly Machinery . . . . . . 6

1.3.2 How Does This Transition of Energy Take Place? . . . . . . . 7

1.3.3 $\alpha$-, $\beta$ - and $\gamma$-SNAP - Specialized or Redundant Isoforms? . . . 8

1.3.4 Input and Output of the Machinery - Which Other Substrates or Products are Feasible? . . . . . . . . . . . . . . . 9

1.3.5 Potential Regulatory Mechanisms of SNARE Disassembly . . 9

1.3.6 The Yeast Homologous Protein Family - How Conserved is the Disassembly Mechanism? . . . . . . . . . . . . . 12

1.3 .7 Summary . . . . . . . . . . . . . . . . 13

1.4 Aim of This Study . . . . . . . . . . . . . . . . . . . . . 13

2 Materials and Methods $\quad \mathbf{1 5}$

2.1 Chemicals . . . . . . . . . . . . . . . . . . . 15

2.2 Chemical solutions . . . . . . . . . . . . . . . . . 16

2.3 Culture media . . . . . . . . . . . . . . . . . . 16

2.4 Bacterial strains . . . . . . . . . . . . . . . . . . . 17

2.5 Enzymes . . . . . . . . . . . . . . . . . . . . 17

2.6 Fluorescent labels . . . . . . . . . . . . . . . . . . . . . . 18

2.7 Instruments . . . . . . . . . . . . . . . . . . . . . . . . . . . . 18

2.8 Constructs . . . . . . . . . . . . . . . . . . . . . . . . . . 18

3 Methods $\quad 21$

3.1 E.coli competent cells . . . . . . . . . . . . . . . . . . 21

3.2 Transformation . . . . . . . . . . . . . . . . 21

3.2.1 Electroporation . . . . . . . . . . . . . . . . . . . 21 
3.2 .2 Heat-shock . . . . . . . . . . . . . . . . 22

3.3 Cloning of the $\alpha$ delSNAP mutant . . . . . . . . . . . . . . 22

3.4 Protein expression and purification . . . . . . . . . . . . . . 22

3.5 Gel Electrophoresis . . . . . . . . . . . . . . . . . . . . . 24

3.6 Gel based disassembly . . . . . . . . . . . . . . . . . . . . . 24

3.6.1 Disassembly of the minimal core complex . . . . . . . . . . . 24

3.7 Protein labeling . . . . . . . . . . . . . . . . . . . . 24

3.8 Fluorescence measurements . . . . . . . . . . . . . . . . . . 25

3.9 Fluorescence anisotropy . . . . . . . . . . . . . . . . . . . 25

3.10 FRET . . . . . . . . . . . . . . . . . . . 25

3.11 Preparation of proteoliposomes . . . . . . . . . . . . 25

3.12 Fluorescence spectroscopy on liposomes . . . . . . . . . . . . . . . . . 26

3.13 Monoclonal antibodies . . . . . . . . . . . . . . . . . . . . 26

3.14 Western Blotting . . . . . . . . . . . . . . . . 26

3.15 Cell Culture . . . . . . . . . . . . . . . . . . 27

3.16 Disassembly on membrane sheets . . . . . . . . . . . . . . . . . . . . . . . . . . 28

3.17 Isothermal titration calorimetry . . . . . . . . . . . . . . . 28

4 Results $\quad 29$

4.1 Basic In Vitro Characterization of the Disassembly Reaction . . . . . 29

4.1.1 Purification of Active, Hexameric NSF . . . . . . . . . . . . . 29

4.1.2 Monitoring Disassembly Via SDS-PAGE . . . . . . . . . . . 30

4.1.3 Online Monitoring of Disassembly Via Fluorescence Spectroscopy 30

4.1 .4 The FRET Assay . . . . . . . . . . . . . . . . . . . . . . . . . 31

4.1.5 Fluorescence Anisotropy as a Readout for Disassembly . . . . 33

4.1.6 Optimizing Protein Amounts for Fast Disassembly Kinetics . . 33

4.2 How robust is the reaction? . . . . . . . . . . . . . 36

4.2.1 Inhibition of the Enzyme . . . . . . . . . . . . . . 37

4.2.2 Influencing the Reaction on the Target Level . . . . . . . . . . 40

4.2.3 Evidence for a Robust Target Recognition of the Disassembly

Machinery . . . . . . . . . . . . . . . . 47

4.2.4 Inhibition on the SNAP Level? . . . . . . . . . . . . . . 51

4.3 Why So Much $\alpha$ SNAP? . . . . . . . . . . . . . . . . . 52

4.3.1 $\alpha$ SNAP Binding to the Complex Monitored Calorimetrically . 52

4.3.2 $\alpha$ SNAP Dependence of SNARE Disassembly on 'Membrane

Sheets' ......................... 54

4.3.3 Disassembly of SNARE Complexes Incorporated into Liposomes 57

4.3.4 NSF Performance is Unchanged on Liposomes . . . . . . . . . 62

4.4 Membrane Anchorage of SNAREs Potentiates the Power of $\alpha$ SNAP . 65

4.4.1 The Transmembrane Domain of Synaptobrevin is not Essential for $\alpha$ SNAP Potentiation . . . . . . . . . . . 65

4.4.2 The Potentiation of $\alpha$ SNAP Efficacy is Independent of the Lipid Composition . . . . . . . . . . . . . . 68 
4.4.3 Disassembly on Partially Solubilized Liposome Populations (Mixed Micelles) . . . . . . . . . . . . . . . . 69

4.4.4 An $\alpha$ SNAP Mutant Lacking the Putative Membrane Interaction 72

4.4.5 Potentiation Through Membrane Anchorage - General Feature of SNAPs or $\alpha$ SNAP-Specific Phenomenon? . . . . . . . . 76

4.5 How is the Reaction Being Regulated? . . . . . . . . . . . . . . . . . 80

4.5.1 Influence of Complexin1 on Core-Complex Disassembly . . . . 80

4.5.2 Does Phosphorylation of NSF Have an Impact on Function? . 84

4.6 How Conserved is the Reaction Mechanism? . . . . . . . . . . . . . . 88

4.6.1 SNARE Disassembly Using Yeast Homologues . . . . . . . . . 88

4.6.2 Disassembly of Neuronal Complexes Using the Yeast Machinery 88

4.6.3 Disassembling the Yeast SNARE Complex . . . . . . . . . . . 90

5 Discussion

5.1 Part 1 - Characterization of the SNARE Reaction Using Fluorescence Spectroscopy . . . . . . . . . . . . . . . 9 96

5.1.1 The Membrane is a SNAP Receptor . . . . . . . . . . . . 100

5.1.2 Summary - Advantages of the Fluorescence-Based Experiments over Conventional Disassembly Assays . . . . . . . . . . 107

5.2 Part 2 - Modulating the Reaction . . . . . . . . . . . . . . . . . 108

5.2.1 Robustness of the Reaction - Bottlenecks and Putative Regulatory Targets . . . . . . . . . . . . . . . . . . . . 108

5.2 .2 Inhibition on the $\alpha$ SNAP Level . . . . . . . . . . . . . . 113

5.2.3 Phosphorylation of NSF as a Regulatory Mechanism? . . . . . 118

5.2.4 Conservation of the Reaction Mechanism in Yeast . . . . . . . 120

6

6.1 Summary . . . . . . . . . . . . . . . . . . . . . 125

6.2 Zusammenfassung . . . . . . . . . . . . . . . . . 127

$\begin{array}{llr}7 & \text { Appendix } & 137\end{array}$

7.1 Abbreviations and Symbols . . . . . . . . . . . . . . . 139

7.2 Eidesstattliche Erklärung . . . . . . . . . . . . . . . . . . . . . 141

7.3 Acknowledgements . . . . . . . . . . . . . . . . 145 


\section{Chapter 1}

\section{Introduction}

To fulfill their specific function most if not all proteins interact with other proteins in some way or another. The assembly of protein complexes is therefore nothing unusual and one might ask why the mechanism of SNARE assembly should be interesting enough to be explained in great detail. The assembly of SNARE complexes is said to mediate fusion of biological membranes. But why is that so fascinating? How does the cell succeed to fuse membranes? How the right ones, at a sufficient speed, at the right times? Which are the key players, what is their mode of action and how are they being regulated? The main purpose of intracellular membranes being the enclosure and thereby division of different subcellular environments, they are usually spatially separated from each other which keeps them from interacting spontaneously. Furthermore, even if two membranes are in close apposition, the repulsion of charges between them still prevents fusion of different compartments from occurring - as long as no additional input of energy is provided. Therefore a reaction bearing the potential to drive membrane fusion obviously needs to fulfill at least two critical criteria: First, it needs to be able to mechanically draw the membranes close to each other, second it needs to generate enough energy to overcome the energetic barrier. These preconditions given, an additional level of complexity is added when we start to think of regulatory functions providing specificity. Tight regulation concerning the amount, speed and type of vesicles prone to fuse is also required to add to the quality of the fusion reaction. Nevertheless these regulatory mechanisms do not necessarily have to be inherent to the basal fusion machinery but could theoretically also be provided by other factors. Keeping these prerequisites in mind the underlying mechanisms bear some fascination. If the two main criteria, pulling membranes together and overcoming the energetic barrier were to be met by proteins - how should these proteins be structured, how would they interact? Since, as stated in the beginning, the SNAREs actually represent a family of proteins capable to fulfill this difficult task, studying their structure and mechanisms of interaction has helped to gain insights into membrane fusion during the last decades 
and probably will continue to do so in the future.

\subsection{Function of the SNARE Proteins}

All intracellular transport processes ranging from secretion in yeast to neurotransmitter release in the brain, depend on the ability of membranes to fuse with each other. Due to the high variability regarding time-scale and function of these various processes, the group of proteins mediating membrane fusion must on the one hand show common characteristics which enable them to execute the event of fusion. On the other hand however, there must be diversities allowing for tight spatial and temporal regulation. SNAREs can be found in all eukaryotic organisms and their involvement in many steps of intracellular transport has been proven using various experimental systems. It is now commonly believed that they are involved in all vesicular fusion events, with different sets of SNAREs being responsible for different trafficking steps [1]. The SNAREs are usually short and C-terminally attached to the membrane by either a transmembrane region or a membrane anchor. One set of SNAREs typically consists of four proteins, which when combined in appropriate solutions spontaneously form extremely stable complexes via a region common to all SNAREs called the 'SNARE motif' or 'core-domain'. The association takes place along a highly exothermic reaction pathway which already early in SNARE research made it tempting to speculate that the assembly of SNARE complexes provides the driving force for membrane fusion $[2,3,4]$. Indeed, later they were shown to be able to autonomously catalyze liposome fusion in vitro without any additional factors [5].

\subsection{A Closer Look at SNARE Assembly}

But how can the assembly of proteins lead to the merger of two membranes? Even though the complete answer to this question has not been conclusively found, some by now well understood basic mechanistic principles of SNARE assembly have been of help to establish different models. One of the concepts of how SNARE assembly actually proceeds, the so called 'zipper'-mechanism, has gained more and more support over the years.

\subsubsection{The 'Zipper'-Model-How Does SNARE Assembly Pro- ceed?}

The zipper model proposes that SNARE proteins residing on opposing membranes first interact at their membrane distal termini to form a loose complex. Starting from this point of interaction they consequently wind up towards their C-terminal 
membrane anchors in a zipper-like fashion to form the four-helix-bundle, bringing the membranes into close proximity (illustrated in figure 1.1). The amounts of energy generated during assembly eventually suffice to allow for the membrane merger. In other words, SNAREs directly function as fusion catalysts $[6,7,8,9]$.

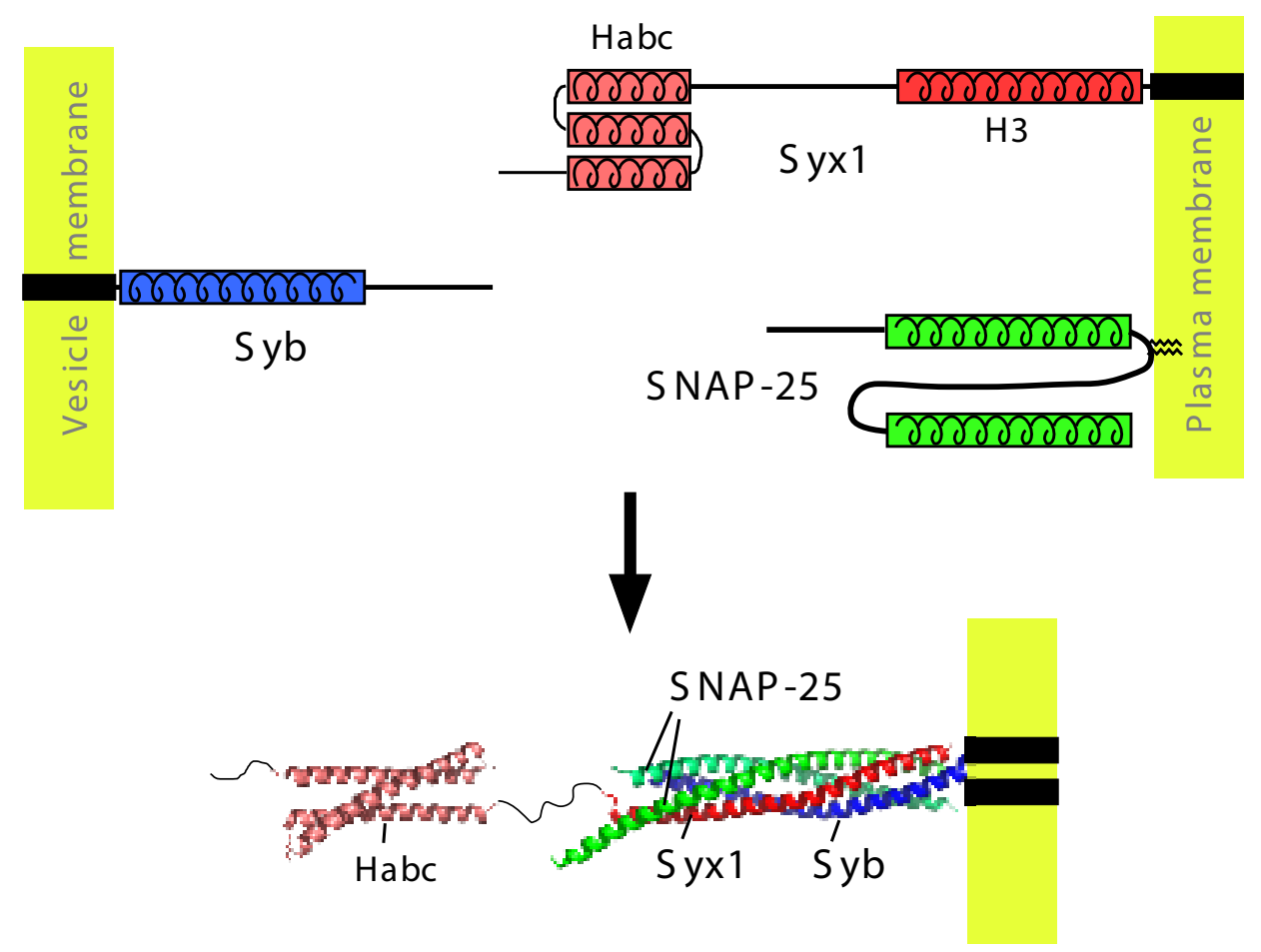

Figure 1.1: SNAREs switch from 'trans' to 'cis' during fusion. The figure exemplary shows the distribution of the neuronal SNAREs before and after fusion. Before fusion, the Q-SNAREs reside on the plasma membrane whereas Synaptobrevin (Syb) is found on the vesicle. After fusion of the two membranes into one, all three SNAREs are depicted in complex, indicating that the complex has assembled during fusion. As described in the text, assembly does not only temporally coincide with but rather is instrumental to drive the fusion reaction. The driving force results from conformational changes which are schematically shown: Before fusion the SNAREs are mostly unstructured whereas during fusion a tight helical bundle is formed.

\subsubsection{The Driving Force-Spontaneous Formation of Ex- tremely Stable Complexes}

Evidence for this model comes from various kinds of experimental systems. The first mechanistic insights resulted from experiments with recombinant neuronal SNAREs in solution. Most of the early experiments were performed using only the SNARE core domains, which were found to be sufficient to mediate SNARE assembly displaying similar biophysical characteristics as the full-length SNARE complex. A 
common characteristic of these motifs is a lack of secondary structure as long as they are in a monomeric state $[3,4,6,7,10,11]$. Only upon mixing with other SNAREs do certain combinations lead to the spontaneous association into complexes accompanied by major conformational and free-energy changes. Even though the two neuronal SNAREs Syntaxin1 and SNAP-25 (synaptosome-associated protein of $25 \mathrm{kDa}$ ) alone form complexes displaying high $\alpha$-helical content, combining all three neuronal SNAREs produces amongst other side-products one uniquely stable outcome, which consists of all three neuronal SNAREs in a 1:1:1 stoichiometry. Not only is it resistant to higher amounts of SDS than all other resulting complexes and extremely high temperature - it also shows a pronounced hysteresis meaning that there is basically no spontaneous dissociation under assembly conditions [12]. Since all other side products are in equilibrium with the reactants, the degree of this respective complex increases with reaction time. Furthermore this ternary SNARE complex can be isolated from brain extracts indicating its existence in vivo [13] making it the most probable end product of the assembly pathway.

\subsubsection{The Logistics - SNAREs Switch from 'trans' to 'cis' During Membrane Fusion}

The SNAREs are in a 'trans'-state as long as fusion is incomplete (residing on opposite membranes). As soon as the membrane merger has taken place and all helices are anchored in one membrane the complex is considered to be in a 'cis'configuration (figure 1.1). Originally the SNAREs were classified into vesicle (v-) and target ( $\mathrm{t}$-)SNAREs, according to their location on the membrane before fusion [14]. The drawback of that nomenclature is however that the functional distribution of some SNARE sets has not been characterized sufficiently to assign vesicle- or target-function and especially when it comes to homotypic membrane fusion this classification meets its limits. By comparison of the neuronal ternary complex crystal-structure to those of two other, only distantly related (endosomal) SNARE complexes, it became evident that all three complexes resemble an intertwined $\alpha$-helical bundle of parallel coiled coil domains consisting of four helices per complex $[15,16,17]$. The parallel alignment of SNAREs in the ternary complex mentioned in the context of the crystal structure was originally observed with the help of FRET- as well as EM-experiments $[8,18]$. These SNARE complexes show a remarkable degree of conservation which led to a reclassification of SNAREs based on a structural property: The centre of the bundle contains 16 stacked 'layers' of interacting side chains which are largely hydrophobic, except for the very central one which contains three highly conserved glutamine (Q) residues and one highly conserved arginine $(\mathrm{R})$ residue. Hence the SNAREs with a glutamine in this so called '0'-layer were grouped Q- and the ones with arginines R-SNAREs. The Q- 
SNAREs can further be divided into Qa-, Qb- and Qc-SNAREs, each of these classes contributing one helix to the four helix bundle [6]. The neuronal SNAREs belong to a unique subset of SNAREs in the sense that rather than being part of two independent proteins, the Qb and Qc-SNARE motifs are accommodated in one SNARE called SNAP-25 (synaptosome-associated protein of $25 \mathrm{kDa}$ ). It is tempting to speculate that it represents a fusion product of two reactants of the assembly reaction which has evolved because it may have led to a kinetic advantage over a fourcomponent reaction. Thinking along these lines one can imagine that neuroexocytic events profit from this advantage possibly contributing to the high speed of fast firing neurons. Syntaxin and Synaptobrevin represent the second neuronal Q-SNARE and the R-SNARE, respectively. The neuronal SNAREs are distributed such that the R-SNARE is primarily located on the vesicle whereas the Q-SNAREs are predominantly located on the cell membrane. If the structural arrangement is a general feature of all SNARE complexes, their localization to the different membranes might also be conserved, but since characterization of different SNARE topologies has not come to an end yet, this at the moment is mere speculation.

According to this hypothesis one membrane would typically contribute three SNARE motifs whereas the second would contribute the fourth helix. On the one hand experiments on neuronal SNAREs and in yeast have led to this hypothesis. On the contrary homotypic in vitro-fusion of liposomes mediated by early-endosomal SNAREs has been shown to proceed with different SNARE topologies regardless of whether all Q-SNAREs are contributed by the same membrane or not [17]. Whether a 3Q/1R-rule holds true for all SNARE sets in a physiological context therefore remains a matter of debate and yet needs to be elucidated. Taken together, these findings are all in accordance with the zipper model: First of all the assembly reaction is exogenous and hence a source of energy, which spontaneously proceeds without additional factors. Second, the SNAREs reside on opposing membranes before, whereas they are found on the same membrane after fusion. Third, the helices are aligned in parallel after assembly, thus the N-termini interact with the $\mathrm{N}$ - whereas the C-termini interact with the C-termini of the partner SNAREs. 


\subsection{Postfusion-Time for Recycling of SNARE Com- plexes}

After assembly, the complexed R- and Q-SNAREs reside in the same membrane and are no longer free to act in further rounds of fusion. As much as the above described characteristics like pronounced hysteresis, exothermal driving force of the reaction and the high level of complex-stability push the reaction into the direction of assembly they of course prevent the complex from voluntary disassembly under physiological conditions. Hence basically no spontaneous dissociation occurs. If there were no counteracting mechanism, all fusion events would come to an end as soon as all free SNAREs were used once. Of course the cell could constantly dispose of the fully assembled SNARE complexes and synthesize new free SNAREs in a single-use fashion, but keeping in mind that during assembly the SNAREs only undergo structural but no degradational changes, one can envision a faster and probably energetically more favorable solution to this problem: A factor which tears the complexes apart despite their apparent stability, e.g., by lowering the activation energy of this thermodynamically unfavorable reaction. The cell has exploited a family of ATPases which due to their functional variability have been assigned 'ATPases associated with various cellular activities (AAA ATPases)', to lower the activation energies of various reactions, mostly connected to folding or unfolding of proteins. One of these ATPases called NSF (N-ethylmaleimide sensitive fusion protein), with the help of its co-factors, the so called SNAPs (soluble NSFattachment proteins), mediates the disassembly of the stable SNARE complexes and thereby recycles the fusion machinery [19]. To do so NSF manages to couple ATP hydrolysis to the highly endothermic dissociation of the 4-helix bundle.

\subsubsection{NSF and the SNAPs - The Disassembly Machinery}

NSF is ubiquitously expressed and therefore most likely capable of disassembling all existing SNARE complexes of a certain species [19]. Its universal mechanism is also underlined by the fact that to a certain extent functionality even between different species seems to be conserved. As an example the NSF yeast homologue Sec18p has been shown to stimulate exocytosis in permeabilized adrenal chromaffin cells [20].

NSF consists of three domains, two of which, termed the D1- and D2-domain, can bind ATP [21]. At one of these binding sites ATP is actively being hydrolyzed to provide energy during disassembly, whereas ATP-binding to the second site functions in oligomerization of the protein $[22,21]$, which is largely hexameric under equilibrium conditions [23].

SNARE complexes do not display any direct binding sites for NSF. In order for it to disassemble the complex, SNAPs are required which provide high affinity 
binding sites for both, the enzyme as well as its substrate [24]. SNAPs therefore serve as connectors between the SNARE complex and NSF which can disassemble the SNARE complex in the presence of $\mathrm{Mg}^{2+} \mathrm{ATP}$ as soon as one or more, most likely three, SNAPs have bound (figure 1.2).

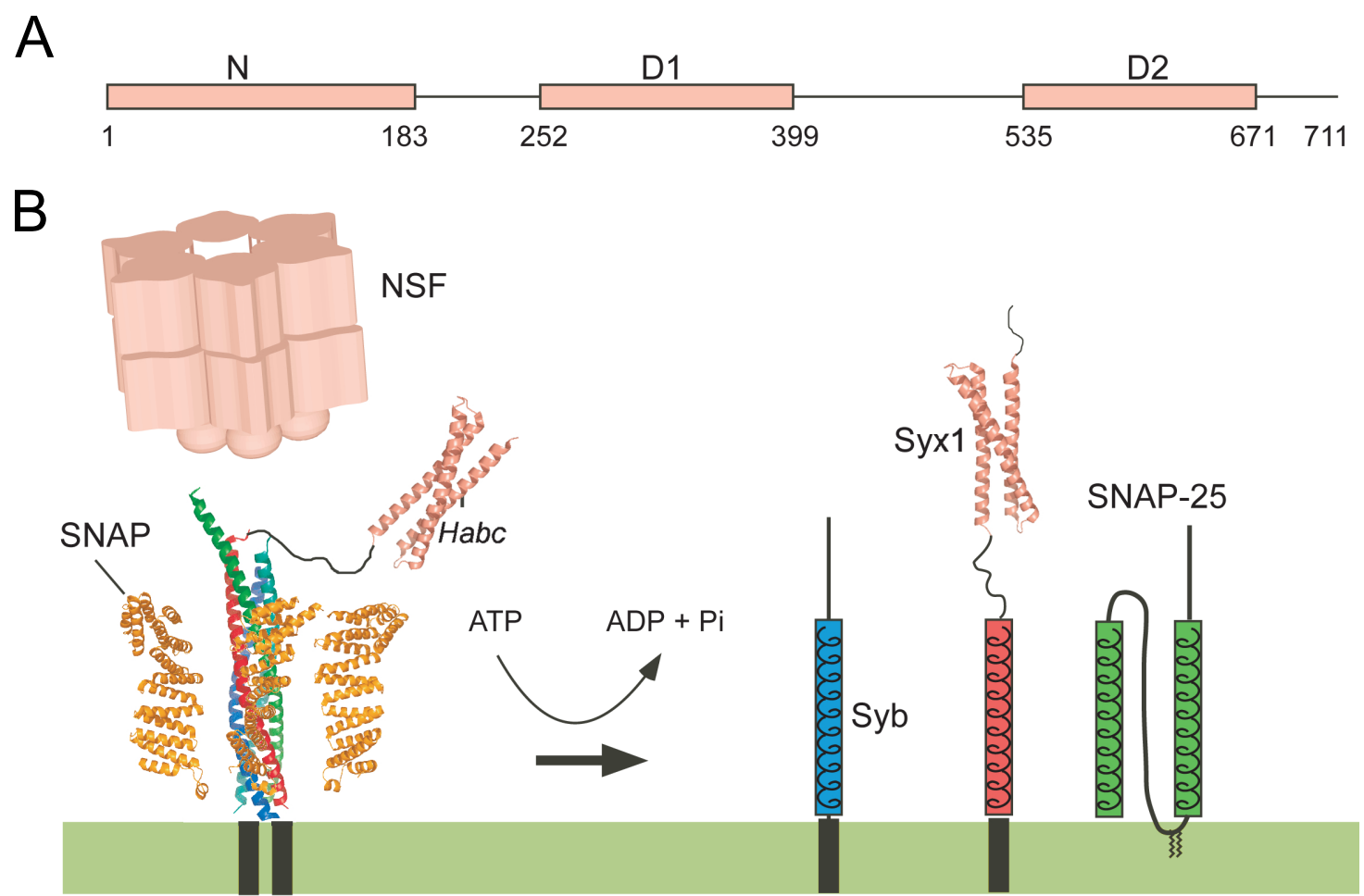

Figure 1.2: Disassembly of SNARE complexes. (A) The NSF-molecule consists of three subdomains which are schematically depicted. The N-domain includes the binding region for $\alpha$ SNAP, the D1 domain actively hydrolyses ATP during disassembly and the D2 domain plays a role in oligomerization of the molecule, which is mostly hexameric under equilibrium conditions.

(B) Most likely three $\alpha$ SNAPs bind to one SNARE complex to form a recognition site for one hexamer of NSF. Since no high resolution structure of NSF in its hexameric state has been solved it is shown schematically. In the presence of $\mathrm{Mg}^{2+} \mathrm{NSF}$ then disassembles the SNARE complex, a process which is fuelled by hydrolysis of ATP into ADP $+\mathrm{P}_{\mathrm{i}}$.

\subsubsection{How Does This Transition of Energy Take Place?}

Compared to the assembly reaction the molecular details of disassembly are much less understood. Electron microscopic images of NSF gave some insight into the mechanism in the sense that clearly distinguishable structures were visible, depending on the respective nucleotide bound [8]. These pictures suggest that hydrolyzing ATP to $\mathrm{ADP}+\mathrm{P}_{\mathrm{i}}$ leads to conformational changes in the whole NSF-hexamer which might exert mechanical force onto the complex it is bound to. 
Hexamers have a donut-like shape, which gives rise to the speculation that the hole in the middle might fulfill some kind of function, especially since other likewise hexameric AAA ATPases are known to use their holes to pull interaction partners through $[25,26]$. On the one hand, it is well imaginable that NSF drags one of the SNAREs through its center pore during disassembly, thereby separating it from the other SNAREs. On the other hand the size of the pore in an NSF-hexamer would theoretically be large enough to accommodate an unfolded protein, like e.g. monomeric synaptobrevin, but according to EM-data meet its limitations as soon as folded domains were to fit through [8]. In some sets of SNAREs each of the four proteins includes one domain with inherent tertiary structure which makes this model improbable unless drastic conformational changes facilitating pore enlargement take place during ATP-hydrolysis.

Alternatively, the NSF-hexamer might, while sitting on the scaffold provided by SNAPs, create a rotational force through conformational changes which moves the SNAPs in a circular manner. The SNAPs being connected to the SNARE complex would insert the same rotational force onto the complex. Since this again is rigidly attached to the membrane it is not difficult to picture that as a consequence the complex would be unwound into its individual SNAREs, like operating a mandrel in the opposite direction would unwind a thread into its individual fibers.

Originally it was widely believed that disassembly proceeds in a 'symmetric' fashion, all six NSF-molecules synchronously hydrolyzing ATP and only then leading to conformational changes in the ring-like structure which consequently enable dismantling of the SNARE complex. More recently an alternative possibility has been raised, which is based on studies undertaken on another AAA-protein called ClpX [27]. Here the authors could show that the single subunits of one ClpXhexamer were capable to exert their catalytic function independently from each other, whereas a concerted action of several subunits apparently increased the enzymatic efficiency in a proportional dependence. If a comparable mechanism were to be utilized by NSF, it would also be conceivable that, depending on the number of NSF-subunits participating in a specific reaction, the amount of $\alpha$ SNAPs per disassembly event is similarly flexible. Nevertheless no attempts have been made to investigate whether NSF-activity also works in an asymmetric manner, or whether all six subunits need to bind and hydrolyze ATP in concerted fashion.

\subsection{3 $\alpha-, \beta$ - and $\gamma$-SNAP - Specialized or Redundant Iso- forms?}

Besides $\alpha$ SNAP two more SNAP-isoforms called $\beta$ SNAP and $\gamma$ SNAP are known [28]. Of all three mammalian SNAPs, the structure has only been solved for $\gamma$ SNAP using crystallographer [29]. Despite limited sequence homology, the $\gamma$ SNAP structure 
strongly resembles that previously solved for the SNAP homolog in yeast, Sec17 [30]. Even though $\alpha \mathrm{SNAP}$ alone is sufficient to serve as a co-factor for disassembly in in vitro experiments, it remains unclear whether the other known SNAP isoforms $\beta$ and $\gamma$-SNAP function as positive or negative regulators or simply provide redundancy despite relatively low sequence homology [31, 32, 33, 34].

In fact so little is known about the mechanism that the number of $\alpha$ SNAPs required is still debated. Three might be needed, but alternatively one or two might also suffice. In any case participation of more than three can be excluded. It still remains unclear how exactly one SNARE complex, one to three $\alpha$ SNAPs, but six NSF molecules (meaning one hexamer) can reasonably be put together. No structure of the complete disassembly machinery, also termed the '20S'-complex [24], has been solved leaving room for speculations.

\subsubsection{Input and Output of the Machinery - Which Other Substrates or Products are Feasible?}

Neither all substrates nor products of the disassembly-reaction are known. For instance SNAREs could theoretically be fully disassembled into monomers but alternatively might also only be partially disassembled up to an intermediate stage. SNAREs contributed by one membrane could for example be kept together in order to decrease the complexity of the subsequent fusion reaction.

Likewise even though there is no doubt that the ternary SNARE complex is a target of NSF, it cannot be excluded that other complexes e.g. assembly intermediates or unwanted dead-end-complexes also serve as substrates for NSF-mediated disassembly. If this were to be the case, NSF might in addition to its indirect effect on SNARE assembly, in terms of SNARE recycling, also directly regulate complex assembly by influencing the steady-state concentrations of complex intermediates.

\subsubsection{Potential Regulatory Mechanisms of SNARE Disas- sembly}

Even though rapid SNARE-complex disassembly is essential for the cell in order to provide sufficient amounts of free SNAREs to readily replenish SNARE pools after fusion, it is nevertheless of high importance that SNARE disassembly, like all vital physiological processes, is tightly regulated. It should for example be granted that uncontrolled disassembly does not deprive the cell of factors which at the same time are needed for other vital processes. $\alpha$ SNAP as well as NSF have been reported to be involved in various other cellular functions independently of each other, making it feasible that there are mechanisms which inhibit disassembly as soon as one of them becomes limiting. Likewise, since ATP is a fuel needed for a large variety of other 
vital cellular processes as well, NSF activity might be restricted under conditions in which ATP levels are low. Furthermore, disassembly of SNARE complexes prior to completion of assembly would, according to current knowledge, prevent final membrane merger and should hence also be regulated by the cell.

SNARE-complex disassembly being mediated by the combined efforts of two proteins brings about the question of whether regulatory mechanisms act on the level of the enzyme, the adaptor or either of them. Finally the SNARE target might also be the targets of regulation. Up to date, several groups found potentially regulating factors, covering all three of these possibilities.

\section{The enzyme - Regulation of NSF activity}

For NSF, nitrosylation and phosphorylation, both having an apparent inhibitory effect on its function, have been reported. Phosphorylation at residue Ser-237 by a Serine/Threonine-kinase supposedly results in a hexameric form which does not bind SNAP/SNARE complexes [35]. Likewise, phosphorylation at the tyrosineresidue 83 has been suggested to lead to reduced binding of $\alpha$ SNAP [36]. In vitro phosphorylation has also been witnessed for $\alpha$ SNAP where it apparently led to a ten-fold reduction in SNARE-complex affinity [37].

\section{The adaptor - Regulation on the level of the SNAP}

Additionally, proteins able to compete with $\alpha$ SNAP for SNARE complex binding have been described. Amongst these are the so called Complexins (also known as Synaphins) which were originally identified by their interaction with the neuronal SNARE core complex [38, 39].

Complexins The Complexins represent a family of small proteins without folding similarities to any other protein family and are present in all multicellular eukaryotes. Complexins have originally been reported to displace $\alpha$ SNAP from SNARE complex when added in excessive amounts, leading to speculations that they might have inhibitory properties with respect to SNARE disassembly [39]. Notwithstanding these findings, in vitro assays directly monitoring SNARE disassembly do not show any negative influence of Complexin 1 or 2 on SNARE-complex disassembly [40]. However, due to a time resolution in the range of several minutes, these experiments are preferably suited for comparing 'all-or-nothing' disassembly defects rather than minor kinetic differences which could hence not be ruled out. Meanwhile the mode of binding between Complexin and the SNARE core complex has been investigated. An $\alpha$-helical central stretch consisting of 58 amino acids was found to bind the SNARE complex in an anti-parallel fashion [41], and the structure of complexin bound to the neuronal SNARE complex has been solved [42]. Seemingly contradictory outcomes 
of knock-out experiments and over-expression studies in various animals and celltypes have complicated the determination of complexin function, leaving a range of proposed models in the field up to now. Most of these studies report a reduction of exocytosis in presence of excess Complexin which would be in line with a negative impact on fusion. However, at the same time the absence of complexin appears to result in impaired exocytosis as well. Recently, three different studies resulted in findings which led to a similar interpretation, according to which Complexin might act as a so called fusion clamp binding the SNARE complex in a partially zippered state prior to complete fusion and thereby 'clamping' it until the signal for the final fusion step is received [43, 44, 45]. Even though this scenario primarily envisions Complexin to function as an inhibitor of the last step of SNARE assembly, it does not exclude the possibility that the 'Complexin clamp' serves as a protection against unwanted disassembly at the same time. Altogether the apparently contradictory results could in principle also be explained by a fine-tuning capability which for instance might depend on a physiological Complexin concentration. If this were to be the case, too little as well as too much Complexin would harm the balanced fusion process and might lead to similar phenotypes. Complexins' probably complex role and possible implications on SNARE disassembly hence require further elucidation.

\section{The membrane - Another check-point of SNARE regulation?}

Even though SNARE complexes readily assemble and with the help of NSF and $\alpha$ SNAP disassemble in solution, they are naturally located on membranes, which might also influence their mechanism as well as speed of assembly or disassembly. SNAREs have been inserted into membranes but only little has been done to dissect the process on membranes in detail. What has been done up to now has led to contradictory results. EPR-measurements have attributed a possible role of the membrane to control the capability of SNARE motifs to enter SNARE complexes. They indicated that a short membrane proximal region of Synaptobrevin-2 is dipped into the membrane and thereby inhibits the formation of SNARE complexes [46], a regulatory mechanism that was called 'Synaptobrevin restriction' which could in this study be overcome by mutation of two membrane proximal tryptophan residues. This scenario would to some extent contradict the results mentioned above, which suggested that SNARE zippering proceeds in an N- to C-terminal direction. Notwithstanding these findings, other groups have witnessed fusion of native Synaptobrevin membranes and Synaptobrevin has recently been shown to be constitutively active, regardless of whether incorporated into membranes or not [47]. Here Synaptobrevin could be driven into SNARE complexes both in isolated synaptic vesicles and in proteoliposomes. Even though the membrane thus does not seem to regulate SNARE assembly as such, it might influence one of the other steps of the SNARE 
cycle. Recently, Munc-13 was found to interact with SNARE complexes when these are incorporated into liposomes, whereas they hardly interacted in solution [48].

Likewise, SNARE disassembly might be affected by the membrane. $\alpha$ SNAP and its yeast homologue Sec17 have for instance been suggested to directly interact with membranes independently of the well established interaction with membrane bound SNARE complexes, which of course also confines them to the membrane $[49,50]$. This is in line with the observation that $\alpha \mathrm{SNAP}$ is an amphipathic proteins which binds to plastic surfaces [51]. Steel et al. report that the stimulation of the NSF ATPase-activity by $\alpha$ SNAP is more pronounced in the presence of lipids [50]. Lastly, NSF is released inefficiently from many cellular membranes under conditions which allow for 20S-complex disassembly. This might be an evidence for NSF-binding to lipids as well. Nevertheless, no experiments directly assessing the impact of membranes on SNARE disassembly have been performed so far, leaving room for further investigations.

\subsubsection{The Yeast Homologous Protein Family - How Con- served is the Disassembly Mechanism?}

Like all other SNARE-complex structures which have been solved so far, the crystal structure of the yeast plasma membrane complex is very similar to that of the known neuronal and endosomal SNARE complexes [52]. The yeast SNAREs constituting the complex are Sso1 (Syntaxin-homologue), Sec-9 (SNAP25-homologue) and Snc1 (Synaptobrevin homologue). Interestingly, the structures of the neuronal and the $S$. cerevisiae complex, both of which are involved in plasma membrane fusion, are even more similar to each other than they are to the endosomal SNARE complexes. As opposed to the neuronal SNARE complex, the yeast SNARE complex seems to be less stable. It neither is SDS resistant nor, with a melting temperature of $55 \mathrm{C}$, is it as heat resistant as the neuronal SNARE complex, which only melts at $\sim 90 \mathrm{C}$.

The homologues of $\alpha$ SNAP and NSF in yeast are Sec17 and Sec18, respectively. The Sec17 structure as well as that of the Sec18 N-terminal have been solved crystallographically [30]. In analogy to the neuronal SNARE complex, in vitro binding studies of Sec17 yeast complex coupled to GST-beads revealed that the stoichiometry of Sec17 to SNARE complex is 3:1 [53]. The crystal structures of the NSF and Sec18 N-terminal domains reveal a remarkable degree of similarity $[54,55,56]$, suggesting that the mode of interaction with their respective SNAP adaptors might also be conserved. As a matter of fact this structural similarity extends to function in the sense that Sec18 can efficiently replace NSF in biochemical and membrane trafficking assays $[57,58,20]$. 


\subsubsection{Summary}

To sum it up, the molecular details of SNARE disassembly remain poorly understood even twenty years after NSF discovery. This is rather astounding, since even though the original hypothesis that $\alpha \mathrm{SNAP} / \mathrm{NSF}$ might be the factors actually mediating the last step of membrane merger has meanwhile been proven wrong by various convincing findings, their nowadays widely acknowledged function as the mediators of SNARE recycling, does not weaken their fundamental importance for intracellular membrane fusion. On the contrary, keeping in mind that the disassembly reaction actually fuels membrane fusion by means of energy transfer into SNAREs one might still consider it as the motor of membrane fusion. It is also conceivable that NSF acts at several steps of the SNARE cycle, on the one hand disassembling SNARE complexes post fusion, on the other hand disassembling complexed SNAREs either not wanted or not able to form fusion competent complexes. Nonetheless it is clear that this motor does not act at the fusion step itself but is temporally uncoupled from actual membrane merger strongly suggesting that there are mechanisms to prevent its action at either the wrong time or on the wrong substrate. Furthermore, since the reaction uses parts of the cellular energy storage, there most likely exist regulatory factors ensuring balanced energy levels at all times. Such modulatory proteins or conditions might either completely abolish or, in other cases, only decrease or enhance the disassembling activity of NSF and/or the SNAPs. Insights into how exactly this might happen may be reached in studies directly investigating the requirements for optimal SNARE disassembly. Comparison of disassembly kinetics under various conditions and in presence or absence of putative regulatory factors might help to shed light on the mechanistic details of the disassembly reaction.

\subsection{Aim of This Study}

The aim of this study was to investigate the molecular basis of SNARE disassembly including SNAP homologues, putative regulatory factors and the degree of functional conservation. Obviously, fine-tuning activities would best be observed using dynamic assays with a high time resolution. Secondly, experiments should be easy to manipulate in a defined manner. Additionally, NSF and $\alpha$ SNAP have been reported to promiscuously interact with several other cellular factors as well, making it difficult to distinguish abnormalities in disassembly from other phenotypic effects possibly resulting from other NSF- and SNAP interactions. Therefore the experiments should be placed in a defined background. Many of the findings collected with regard to $\alpha$ SNAP and NSF so far are based on findings in living cells.

Others have used biochemical assays, which either determine the binding properties of the involved proteins or NSF activity by ATP consumption. Both of these 
biochemical approaches are indirect in the sense that they do not allow for conclusions concerning the actual dissociation of the SNARE complex.

Others have monitored SNARE disassembly in vitro using SDS page, which is a direct read-out, albeit with only limited time resolution [6]. A dynamic in vitro system allowing for direct monitoring of SNARE interaction would therefore be ideally suited for quantitative determination of disassembly kinetics and putative influencing factors. Since fluorescence spectroscopy of SNARE proteins is well established in our lab, it was the most obvious option to also employ fluorescence spectroscopy for the investigation of their disassembly.

Moreover, the experimental set-up should then be exploited to gain further insights regarding the disassembly mechanism, in particular on which parts or interactions are the bootlenecks of the reaction and thus might represent regulatory targets.

In order to be able to also assess regulatory mechanisms and factors which do not act in an 'all-or-nothing' but rather a modulatory fashion, the conditions during the experiment should be as close to nature as possible. For this it is e.g. desirable, that none of the proteins involved is required in unreasonably high amounts for optimal function. As a prerequisite to addressing regulatory and mechanistic questions on the level of all proteins involved, this in vitro system should thus be optimized in such a way that ATP, $\alpha$ SNAP and NSF can be applied in quantities one would expect according to stoichiometric (or enzymatic) considerations or, if known, endogenous concentrations.

Subsequently, the impact of some regulatory factors should be addressed. Finally, the degree of functional conservation of the disassembly machinery was to be investigated with the help of yeast SNARE proteins and their disassembly machinery. 


\section{Chapter 2}

\section{Materials and Methods}

\section{$2.1 \quad$ Chemicals}

Acrylamide/Bisacrylamide solution, Roth (Karlsruhe, Germany)

Agarose (low EEO), Applichem (Darmstadt, Germany)

Ammoniumpersulfate (APS), Sigma (Deisenhofen, Germany)

ATP

Bradford-Reagent, Biorad (Richmond, USA)

Calcium chloride dihydrate, Fluka (Switzerland)

Coomassie Brilliant Blue R-250, Serva (Heidelberg, Germany)

Dipotassium hydrogen phosphate, Merck (Darmstadt, Germany)

Disodium hydrogen phosphate, Merck (Darmstadt, Germany)

Dithiothreitol (DTT), Roth (Karlsruhe, Germany)

EDTA Titriplex III, Merck (Darmstadt, Germany)

Glycine, Sigma (Deisenhofen, Germany)

HEPES (N-(2-hydroxyethyl) piperazine - N-2-ethanesulphonicacid)

Imidazole, Sigma (Deisenhofen, Germany)

Isopropyl-D-thiogalactoside (IPTG), Roth (Karlsruhe, Germany)

Magnesium chloride, Sigma-Aldrich (St.Louis, USA)

MPD (2-methyl-2, 4 pentanediol), Fluka (Switzerland) 
PMSF (paramethyl sulphonyl fluoride), Roth (Karlsruhe, Germany)

Potassium dihydrogen phosphate, Merck (Darmstadt, Germany)

Sodium hydrogen carbonate, Merck (Darmstadt, Germany)

Sodium chloride, Merck (Darmstadt, Germany)

Sodium cholate, Sigma-Aldrich (St.Louis, USA)

Sodiumdodecylsulfate (SDS), Biorad (Richmond, USA)

TEMED (N, N, N, N- Tetramethylethylenediamine), Biorad (Richmond, USA)

Tricine, Applichem (Darmstadt, Germany)

Tris (hydroxymethyl)-aminomethane (Tris), Merck (Darmstadt, Germany)

Triton X-100, Merck (Darmstadt, Germany)

Tween - 20, DAKO (Carpinteria, USA)

Urea, Merck (Darmstadt, Germany)

\subsection{Chemical solutions}

Acetic acid, Merck (Darmstadt, Germany)

Hydrochloric acid, Merck (Darmstadt, Germany)

Isopropanol, Merck (Darmstadt, Germany)

Methanol, Merck (Darmstadt, Germany)

\subsection{Culture media}

Tryptone, Roth (Karlsruhe, Germany)

Yeast extract, Roth (Karlsruhe, Germany)

Agar-agar, Roth (Karlsruhe, Germany) 
LB (Luria Bertani) media: 10g tryptone, 5g yeast extract and $5 \mathrm{~g} \mathrm{NaCl}$, add 1 liter with distilled water added $12 \mathrm{~g}$ agar for solid media.

TB (Terrific broth) media: $13.3 \mathrm{~g}$ tryptone, $26.7 \mathrm{~g}$ yeast extract, $4.4 \mathrm{ml}$ glycerol.

TB salt: 0.17M KH2PO4, 0.72M K2HPO4.

Before inoculation, the media were autoclaved and brought to room temperature. In the case of TB media, after autoclaving, TB salt was added.

\subsection{Bacterial strains}

The following strains were obtained from (Stratagene, La Jolla, CA, USA):

Escherichia coli BL21 (DE3) B F- dcm ompT hsdS (rB- mB-) gal(DE3)

Escherichia coli XL-1-Blue recA1, endA1, gyrA96, thi-, hsdR17, (rk-, mk+), supE44, relA1, lac-, [F', traD36, proAB, lacIqZ_M15, Tn10 (tetr)]

Escherichia coli M15[pREP4] Escherichia coli TBK1 B F dcm ompT hsdS(rB mB) gal (DE3) [pTK Tetr]

\subsection{Enzymes}

Restriction endonucleases, New England Biolabs (Ipswich, USA) or MBI Fermentas (Ontario, Canada)

Lyzozyme, Applichem (Darmstadt, Germany)

DnaseI, Applichem (Darmstadt, Germany)

Thrombin, Merck (Darmstadt, Germany) 


\subsection{Fluorescent labels}

Oregon Green(r) 488 iodoacetamide, Invitrogen (USA)

Texas Red(r) C5 bromoacetamide, Invitrogen (USA)

Alexa Fluor(r) 594 C5 maleimide, Invitrogen (USA)

\subsection{Instruments}

AEKTA explorer, Amersham Pharmacia Biotech (Uppsala, Sweden)

Fluoromax-2, Jobin Yvon-Spex (Edison, NJ, USA)

Flurolog, Jobin Yvon-Spex (Edison, NJ, USA)

SMART chromatography sysyem, GE Healthcare VP-ITC, Microcal

UV-spectrophotometer, UV-2401 PC Shimadzu (Japan)

Luminescent image analyzer, LAS-1000 Fujifilm (Japan)

\subsection{Constructs}

The neuronal SNARE proteins used in the study were Syntaxin1a, SNAP-25A and Synaptobrevin2 and various deletion mutants as indicated in the results section. Norway rat (Rattus norvegicus) cDNAs, made from the transcript encoding these proteins, were used to make the expression constructs. The yeast SNARE proteins were soluble portion of Sec9, Sso1p, and Snc2 as well as transmembrane variants of Snc2 and Sso1. The SNAPs used in this study were $\alpha$ SNAP and mutants, $\beta$ SNAP and Sec17. The AAA ATPases used in this study were chinese hamster NSF, Sec18, NSFY83E.

The following expression constructs were used: 
- Syntaxin variants

- H3 domain of Syntaxin1a (residues 180-262),

- full-length soluble Syntaxin (1-262).

- H3 with the transmembrane region (residues 183-288),

- Syntaxin with transmembrane region (1-288),

- single-cysteine mutant (183-262 cys 225).

- SNAP25A variants

- cysteine-less variant of SNAP25A (residues 1-206),

- partial SNAP25A (residues 1-197),

- partial SNAP25A (residues 1-188),

- point-mutated SNAP25A (residues 1-206, M71A/I192A),

- single-cysteine mutant (residues 1-202 cys 130).

- Synaptobrevin variants

- partial Synaptobrevin (residues 1-96),

- partial Synaptobrevin (residues 1-70),

- partial Synaptobrevin (residues 1-116)

- single-cysteine mutants (residues 1-96 cys 28 and residues 1-96 cys 61).

- Sec9 variants

- partial Sec9 (residues 403-651),

- single-cysteine mutant Sec9 (residues 403-651, cys 587)

- Snc2 variants 
- partial Snc2 (residues 1-93) with and without TMD,

- single-cysteine mutant Snc2 (residues 1-93 cys 24).

- Sso1

- partial Sso1 (residues 179-264) with and without TMD.

- $\alpha$ SNAP variants

- partial $\alpha$ SNAP (residues 1-295),

- $\alpha$ SNAP L295A (residues 1-295),

- $\alpha$ delSNAP (residues 33-295) in pET28a vector (His tag).

- $\beta$ SNAP (residues 1-298) in the pGex-2T vector (GST tag).

- Sec17p (residues 1-292) in a pQE vector (His tag).

- NSF variants

- Chinese hamster ovary NSF (residues 1-744) in pET28a (His tag),

- Chinese hamster ovary NSF (residues 1-744, Y83E) in a pET vector (His $\operatorname{tag})$.

- Sec18p (residues 1- 758) in a pQE vector (His tag).

All the SNARE proteins were in his-tag pET-expression vectors (Novagen). 


\section{Chapter 3}

\section{Methods}

\subsection{E.coli competent cells}

The electrocompetent cells were made employing the following procedure; $10 \mathrm{ml}$ of overnight grown E.coli culture were added to $1 \mathrm{~L} \mathrm{LB}$ medium. The culture was grown at $37 \mathrm{C}$ in a shaker until an O.D. of about $0.5-0.7$ was reached. The cells were later pelleted and resuspended for two consecutive times in 1L and $500 \mathrm{ml}$ ice-cold HEPES buffer (1mM HEPES, pH-7.0), respectively. The cells were again pelleted and were resuspended in 20ml HEPES buffer with $10 \%$ (v/v) glycerol followed by pelleting and resuspension in $3 \mathrm{ml} 10 \%(\mathrm{v} / \mathrm{v})$ glycerol. They were frozen rapidly in liquid nitrogen. The cells were later stored at $-80 \mathrm{C}$. The heat-competent cells were made as described in [95].

\subsection{Transformation}

\subsubsection{Electroporation}

The electrocompetent cells were thawed on ice. Then 20ng of plasmid DNA were added and the reaction kept on ice for $10 \mathrm{~min}$. The cells were then transferred to a $0.2 \mathrm{~cm}$ electroporation cuvette (Bio-Rad). The electroporation was carried out with a gene pulser (Bio-Rad) with an $25 \mathrm{~F}, 1.8 \mathrm{kV}$, and 200 ohms pulse. After electroporation $800 \mu \mathrm{l} \mathrm{LB}$ medium were added to the cuvette. The cells were transferred to a microfuge tube and were grown at $37 \mathrm{C}$ for $40 \mathrm{~min}$ in a shaker. Then 50 microliter of the culture were plated on an LB agar plate that contains the appropriate antibiotic for selecting the transformed cells. The plates were kept overnight at $37 \mathrm{C}$ to get transformed colonies. 


\subsubsection{Heat-shock}

An aliquot of the heat-competent cells was thawed on ice and 30ng plasmid DNA were subsequently added. It was incubated for $20 \mathrm{~min}$ on ice. The cells were then subjected to heat shock by keeping them for $90 \mathrm{~s}$ in a water bath that was kept at $42 \mathrm{C}$. The cells were then kept on ice for 1 min until 500 microliter of LB medium were added. The cells were grown at $37 \mathrm{C}$ for $40 \mathrm{~min}$ in a shaker. 50 microliter of the cells were plated on LB agar plates with the appropriate antibiotic and the plates were kept overnight at $37 \mathrm{C}$ to get the transformed colonies.

\subsection{Cloning of the $\alpha$ delSNAP mutant}

The $\alpha$ SNAP mutant $\alpha$ delSNAP (residues 33-295 of $\alpha$ SNAP) was first amplified from $\alpha$ SNAP wild-type DNA using appropriate primers. Afterwards, the PCR product was cleaned up, cleaved with Nde1 and Xho1 and finally ligated into the Nde1 Xho1 cleaved empty pET28a vector (all according to the protocols available online at the Fermentas webpage). The sequence was confirmed by sequencing.

\subsection{Protein expression and purification}

All proteins were expressed as follows: LB medium was inoculated with the appropriate transformed colony of the E. Coli-respective strain. The cells were grown overnight in a shaker at 37C. For expression, $20 \mathrm{ml}$ of overnight grown culture were used to inoculate $1 \mathrm{~L}$ of appropriate antibiotic containing TB or LB medium, the cells were grown until they reached an OD600 of 0.9. They were then induced to express the protein by the addition of IPTG (60 $\mathrm{mg}(\mathrm{w} / \mathrm{v})$ per liter). The cells were grown for 3 hours at $37 \mathrm{C}$ to allow expression of the recombinant protein.

The cells were pelleted and resuspended in $20 \mathrm{ml}$ of resuspension buffer $(20 \mathrm{mM}$ Tris pH-7.4, 1mM EDTA, 500mM NaCl for the SNARES and the SNAPs, or HEPES pH-7.4, 500mM NaCl, 0.5mM ATP, $2 \mathrm{mM} \mathrm{MgCl}$, for NSF, $\mathrm{NSF}^{Y 83 E}$, phosphorylated $\mathrm{NSF}$ and Sec18). The cells were lysed with the combination of lyzozyme treatment and sonication. To reduce the viscosity of the solution DNAse was added; PMSF $(200 \mathrm{mM})$ was added to inhibit the activity of proteases. The lysate was centrifuged at $13,000 \mathrm{~g}$ to pellet the cell debris. The supernatant was used for the affinity purification. All the proteins were soluble and contained a cleavable His- or GST tag for affinity purification.

Affinity Purification Purification of His-tagged proteins ( $\alpha$ SNAP, sec17, all SNAREs, all triple A ATPases): In the case of His-tagged proteins Ni2+-nitrilotriacetic acid beads (Qiagen) (1ml/1l of expressed culture) were added for affinity purification. The beads were washed with excess of wash buffer $200 \mathrm{ml}$ (20mM Tris pH -7.4, $500 \mathrm{mM} \mathrm{NaCl}$, and $8 \mathrm{mM}$ imidazole (+ additionally $0.5 \mathrm{mMATP}, 2 \mathrm{mM} \mathrm{MgCl}_{2}$ in case of the ATPases). The protein was eluted using two bead volumes of elution buffer 
(20mM Tris PH-7.4, 500mM NaCl, 400mM imidazole (plus additionally 0.5mMATP and $2 \mathrm{mM} \mathrm{MgCl}_{2}$ in case of the ATPases)). The His-tag was always cleaved during the subsequent dialysis by the addition of thrombin (100U per 12 liter of culture medium during expression).

Purification of GST-tagged proteins ( $\beta$ SNAP): For $\beta$ SNAP, GST-beads (Qiagen, $2,5 \mathrm{ml} / 6 \mathrm{l}$ of expressed culture) were added for affinity purification. The beads were washed with excess of wash buffer $200 \mathrm{ml}(20 \mathrm{mM}$ Tris $\mathrm{pH}-7.4,500 \mathrm{mM} \mathrm{NaCl}, 1 \mathrm{mM}$ DTT and 0.5mM EDTA). Subsequently, the beads were transferred to a falcon tube using approx. $5 \mathrm{ml}$ of washing buffer and the protein was directly cleaved on the beads by addition of Thrombin (100u) and incubation o.n. Using two bead volumes of wash buffer the cleaved protein was seperated from the beads via a column the next morning.

The affinity purification was followed by a 2nd step of chromatography on an AEKTA system (Amersham Biosciences).

Second step of Chromatopraphy This second step of Chromatography was ion-exchange chromatography for all the SNAREs and all the SNAPs (isoforms, homologs and mutants) and gel-filtration for the ATPases (NSF, pNSF, and $\mathrm{NSF}^{Y 83 E}$ ). Prior to ion exchange chromatography the SNAPs and SNAREs were dialyzed against a buffer containing 20mM Tris pH-7.4, 1mM EDTA, 1mM DTT and 50mM $\mathrm{NaCl}$. The dialysis of the ATPases prior to gelfiltration contained 50mM HEPES, 0,5mM ATP, 2mM EDTA, 200mM NaCl, 2mM DTT and $10 \%$ glycerol. The same buffer was used for gelfiltration via a superdex200 column. Sec18 activity could not be reproducibly recovered after gelfiltration and Sec18 preparations were hence used directly after the Ni/NTA affinity procedure for experiments.

All the proteins obtained after these purification procedures were more than $95 \%$ pure as seen from the SDS-PAGE except from Sec17 and Sec18 (see results). All proteins were quantified either by their absorption at $280 \mathrm{~nm}$ or using the Bradford assay (ATP interferes with the absorption at 280nm).

The yeast SNARE proteins were kindly provided by Xiong Chen and the transmembrane SNARE proteins by Ursel Ries and Alexander Stein.

in vitro phosphorylation of NSF For in vitro phosphorylation of NSF a special E.Coli strain was used. This strain, E.Coli Tkb1, harbors a second plasmid which codes for a tyrosine kinase. NSF was first expressed like usual, except that tetracycline $(12,5 \mu \mathrm{g} / \mu \mathrm{l})$ was included in all bacterial media to select for the plasmid harboring the kinase. After three hours of expression, cells were peletted and resuspended in TK-induction medium as per the manufacterer's instructions (Stratagene) Cells were then grown to an optical density of 0.5 (Abs600) and harvested as described above.

Assembly and purification of SNARE complexes. For the formation of a ternary complex the proteins Syntaxin (or its H3 domain): SNAP25 (or its mutants) 
and Synaptobrevin (or its mutants) were assembled in a 1:1:1.5 molar ratio. They were incubated at room temperature for 2 hours before purification on a Mono-Q column to separate the unassembled monomers from the complex. The proteins were quantified using absorption at $280 \mathrm{~nm}$.

\subsection{Gel Electrophoresis}

For the SDS gel electrophoresis the protocol, as suggested by Laemmli.U.K [59] was used. After electrophoresis the stacking gel was discarded and the separation gel was fixed and stained for $\sim 20$ min in staining solution $(50 \%(\mathrm{v} / \mathrm{v})$ methanol, $10 \%$ $(\mathrm{v} / \mathrm{v})$ acetic acid and $0.2 \%(\mathrm{w} / \mathrm{v})$ Coomassie Brilliant Blue R-250) under agitation. The gel was destained for $\sim 20 \mathrm{~min}$ in Destain $1(20 \%(\mathrm{v} / \mathrm{v})$ isopropanol and $10 \%$ $(\mathrm{v} / \mathrm{v})$ acetic acid) for $\sim 20 \mathrm{~min}$ and then in Destain $2(5 \%(\mathrm{v} / \mathrm{v})$ methanol and 12. $5 \%(\mathrm{v} / \mathrm{v})$ acetic acid) until no background staining was visible. After scanning, the gel was dried in a gel dryer and preserved for further analysis.

\subsection{Gel based disassembly}

\subsubsection{Disassembly of the minimal core complex}

Purified minimal core complex (2.4M) was disassembled by addition of equal concentrations of NSF and a 14-fold excess of -SNAP, $2 \mathrm{mM} \mathrm{MgCl} 2$, and $2.5 \mathrm{mM}$ ATP in 50mM HEPES, pH 7.4, 120mMKGlu/20mM KAc, for 1, 2, 5 and 10 minutes at $37 \mathrm{C}$. The reaction was stopped by adding SDS sample buffer. As a control, the ATPase activity of NSF was abolished by replacing $\mathrm{MgCl}_{2}$ with $10 \mathrm{mM}$ EDTA. All samples were analyzed by SDS-PAGE and Coomassie Blue staining. Unless heated, the minimal core complex (MC) runs as a single band of $34 \mathrm{kDa}$ and thus can be separated from the monomeric SNARE components generated during disassembly.

\subsection{Protein labeling}

The single cysteine mutants were labeled using the sulphahydryl-reactive fluorophores Oregon Green 488 iodoacetamide, Texas Red C5 bromoacetamide, or Alexa Fluor 594 C5 maleimide (Molecular probes- Invitrogen). The proteins were labeled as per manufacturer's instructions. After labeling the labelled protein was separated from the dye by size exclusion chromatography on a Sephadex G-25 column (Amersham biosciences). The labelling efficiency was later calculated according to the manufacturer's instructions. 


\section{8 $\quad$ Fluorescence measurements}

The fluorescence measurements were carried out in the Flurolog-3 or Fluoromax -2 spectroflurometer (Jobin Yvon). A fluorescence cuvette with magnetic stirrer, with a path length of $10 \times 4 \mathrm{~mm}$ or with a pathlength $10 \times 10 \mathrm{~mm}$ (Hellma) was used in the experiments. If not stated otherwise in the figure legend, all the measurements were taken in buffer containing $\operatorname{HEPES}(50 \mathrm{mM}) / \mathrm{KGlu}(120 \mathrm{mM}) / \mathrm{KAc}(20 \mathrm{mM})$, and an additional $0,5 \%$ of chaps in case of the FRET experiments. Whenever disassembly was monitored, the buffers additionally included $2 \mathrm{mM}$ ATP and $5 \mathrm{mM} \mathrm{MgCl}_{2}$ if not stated otherwise.

\subsection{Fluorescence anisotropy}

The fluorescence anisotropy of proteins labelled with Texas red or Alexa594 was measured with excitation and emission wavelengths, $590 \mathrm{~nm}$ and $610 \mathrm{~nm}$ respectively. For an Oregon green labeled protein, excitation and emission wavelengths of 488nm and $520 \mathrm{~nm}$ were used. The $\mathrm{G}$ factor was calculated using $\mathrm{G}=\mathrm{IHV} / \mathrm{IHH}$. The anisotropy $(\mathrm{r})$ was calculated using $\mathrm{r}=(\mathrm{IVV}-\mathrm{G} \times \mathrm{IVH}) /(\mathrm{IVV}+2 \times \mathrm{G} \times \mathrm{IVH})$. Here,'I' refers to the fluorescence intensity, the first subscript is the direction of the exciting light and the second the direction of the emitted light.

\subsection{FRET}

Disassembly :Foerster resonance energy transfer (FRET) was monitored between proteins labelled with Oregon green and proteins labelled with Texas red. The labelled proteins were used at the concentrations indicated in the respective figure legends. The donor, Oregon green was excited at 488nm, the donor fluorescence and acceptor fluorescence (that of Texas red) were measured simultaneously at $520 \mathrm{~nm}$ and $610 \mathrm{~nm}$ respectively.

\subsection{Preparation of proteoliposomes}

Lipids (Avanti, Alabaster, AL) were mixed in chloroform to yield molar ratios as follows:

Standard liposomes: phosphatidylcholine(5),phosphatidylethanolamine(2),phosphatidylserine (1) and cholesterol (1). PE/PC-liposomes: PE(1) to PC(4) PC liposomes: PC only

After drying, the lipids were resuspended in $20 \mathrm{mM}$ HEPES/KOH pH 7.4100 $\mathrm{mM} \mathrm{KCl}, 5 \mathrm{mM}$ DTT, $5 \%$ (w/v) sodium cholate at a total lipid concentration of 13.5 mM. SNARE-proteins in 1\% chaps were added (lipid to protein ratio of 200:1 n/n); followed by size-exclusion chromatography on a PC 3.2/10 fast desalting column (GE Healthcare) with a sample/column volume ratio of $1: 15$, pre-equilibrated in 20 mM HEPES/KOH pH 7.4, 150 mM KCl, 1 mM DTT. 
For the preparation of NBD/Rhodamine labeled liposomes 1.5\% (n/n) 1,2-Dioleylsn-Glycero-3-Phosphoethanolamine-N-Lissamine Rhodamine B Sulfonyl, 1.5\% (n/n) 1,2-Dioleyl-sn-Glycero-3-Phosphoethanolamine-N-(7-nitro-2-1,3-benzoxadiazol-4-yl) and $17 \%$ (n/n) phosphatidylethanolamine were used instead of $20 \%$ (n/n) phosphatidylethanolamine.

\subsection{Fluorescence spectroscopy on liposomes}

Disassembly 10 to 20 microliter of labeled liposomes were diluted in a total volume of $1.0 \mathrm{ml}$ disassembly buffer $(\operatorname{HEPES}(50 \mathrm{mM}) / \mathrm{KGlu}(120 \mathrm{mM}) / \mathrm{KAc}(20 \mathrm{mM})$, $2 \mathrm{mM}$ ATP and $5 \mathrm{mM} \mathrm{MgCl}_{2}$ if not stated otherwise) resulting in final protein concentrations of approx. 35nM to $100 \mathrm{nM}$ (indicated in the respective figures). FRET or fluorescence anisotropy were measured and normalized as described above and at the respective figures.

Detergent titration into liposomes $20 \mu \mathrm{l}$ of Nbd/Rho liposomes were were diluted in a total volume of $1.0 \mathrm{ml}$ buffer $(\operatorname{HEPES}(50 \mathrm{mM}) / \mathrm{KGlu}(120 \mathrm{mM}) / \mathrm{KAc}(20 \mathrm{mM}) / 2 \mathrm{mM}$ ATP and $5 \mathrm{mM} \mathrm{MgCl}_{2}$ ). Dodecylmaltoside was then titrated to the reaction at steps of $62,5 \mathrm{nM}$ per addition and the emission spectra recorded. To excite the Nbd, an excitation wavelength of $460 \mathrm{~nm}$ was used and the emissions of Nbd and Rhodamin were recorded at $538 \mathrm{~nm}$ and $585 \mathrm{~nm}$, respectively.

\subsection{Monoclonal antibodies}

A monoclonal antibody was generated against the NSF N-terminal domain (residues 1-205). The rabbits were immunized with $300 \mathrm{~g}$ of protein emulsified in Freud's adjuvant. It was followed by booster injections that were given every three weeks for 3 to 4 months. Eventually, the antiserum was obtained from the ear veins. Selection of clones was done in cell culture. Finally, the three single clones used for the experiments $(141.1,141.2$ and 141.3) were amplified and concentrated using a bioreactor.

\subsection{Western Blotting}

For semi-dry western blotting the proteins were transferred to a nitrocellulose membrane after gel electrophoresis. The gel and the membrane were sandwiched between blotting papers (GB003 from Schleicher \& Schuell). Before blotting, the blotting papers and the membrane were soaked in transfer buffer $(25 \mathrm{mM}$ Tris/HCl pH-8.3, $193 \mathrm{mM}$ glycine, $20 \%$ (v/v) methanol, $0.1 \%(\mathrm{w} / \mathrm{v})$ SDS). The transfer was carried out at $50 \mathrm{~mA}$ constant current for 1 hour. After transfer, the membrane was either stored at $4 \mathrm{C}$ or it was directly proceeded with blocking. To the membrane, blocking solution $(5 \%(\mathrm{w} / \mathrm{v})$ dry milk powder in PBS) was added and kept in a shaker for 30 
min. The membrane was then washed three times, 10 min each, using the washing solution $(0.1 \%$ Tween-20, 200mM Tris- $\mathrm{HCl} \mathrm{pH} 7.7,0.15 \mathrm{M} \mathrm{NaCl})$. After washing, the primary antibody, which was diluted 1000 times in blocking solution, was added to the membrane. It was either left overnight in a shaker at $4 \mathrm{C}$ or kept in a shaker for 1 hour at room temperature. To remove unspecifically bound antibodies the membrane was washed three times, $10 \mathrm{~min}$ each, with washing solution. After washing, the secondary antibody that was diluted 2000 times in blocking solution was added. The membrane was kept in a shaker for $30 \mathrm{~min}$ at room temperature. The membrane was washed three times, 10 min each, with washing solution to remove the unbound secondary antibody. The secondary antibody was coupled to horseradish peroxidase which emits luminescence after the addition of chemiluminiscence reagent (Perkin Elmer). The luminescence was detected using the luminescent image analyzer.

\subsection{Cell Culture}

Rat pheochromocytoma (PC12, clone 251) cells were grown in $75 \mathrm{~cm} 2$ tissue culture flasks which contained $25 \mathrm{ml}$ of growth medium - DMEM with high $(4.5 \mathrm{~g} / \mathrm{l})$ glucose (Cambrex, New Jersey, USA). DMEM was supplemented with $10 \%$ horse serum (Biochrom, Berlin), $5 \%$ foetal calf serum Gold, $4 \mathrm{mM}$ Lglutamine, $60 \mathrm{U} / \mathrm{ml}$ penicillin, $60 \mathrm{~g} / \mathrm{ml}$ streptomycin (both from Cambrex) which all have been sterile filtered. For maintenance of a Mycoplasma-free cell culture, $6.25 \mathrm{~g} / \mathrm{ml}$ of Plasmocin (InvivoGen, San Diego, USA) was present in cell culture throughout entire period of its growth. Cells were grown at 37C in $10 \%$ CO2 at 90\% relative humidity. For passaging, one flask of confluent cells (approximately 240000 cells/cm2) was used. Growth medium was decanted followed by wash with $3 \mathrm{ml}$ of trypsin/EDTA solution (Cambrex). Cells were detached from their substrate with $3 \mathrm{ml}$ trypsin/EDTA. Trypsin activity on cells was blocked by addition of $27 \mathrm{ml}$ of growth medium. Suspension of cells was centrifuged at $235 \mathrm{x}$ gav for $5 \mathrm{~min}$ at 20C (Varifuge 3.0R, Heraeus-Kendro Sepatech, Langenselbold, Germany). Medium was removed and the resulting cell pellet was titurated in $10 \mathrm{ml}$ of fresh growth medium. Cells were diluted at 1:2 1:4, transferred into $75 \mathrm{~cm} 2$ uncoated tissue culture flasks (Sarstedt, Nuembrecht, Germany) and passaged every 48-96 hrs. To avoid any undesired age-dependent differentiation of culture, cells were used for only 20 passages after defrosting. For seeding on glass coverslips, cells were detached from their substrate as described above, titurated and diluted to give a final concentration of approximately 600,000 cells $/ \mathrm{ml}$. $500 \mathrm{l}$ of this suspension $(3 \times 104$ cells $/ \mathrm{cm} 2)$ were evenly plated onto each poly-L-lysine-coated coverslip. After plating, cells were transferred to incubator and allowed to settle onto the coverslips for at least $30 \mathrm{~min} .3 \mathrm{ml}$ of growth medium were then added. Cells were used for experiments 48-72 hrs after plating. 


\subsection{Disassembly on membrane sheets}

Adherent cells are cultured on cover slips and subsequently subjected to a gentle ultrasound pulse of $100 \mathrm{~ms}$, which 'unroofs' the cells. Fluorescent cis-complexes were generated by reacting membrane sheets with Alexa594-labelled synaptobrevin 2 (lacking its TMR) that forms complexes with membrane sheet associated syntaxin 1 and SNAP-25. Membranes were then directly fixed or fixed after incubation for the indicated times in presence of variable concentrations of NSF and excess of $\alpha-\operatorname{SNAP}(2 \mu \mathrm{M})$, or variable concentrations of $\alpha-\operatorname{SNAP}$ and excess of NSF (40nM) as indicated in the respective figures. Remaining fluorescence after disassembly was related to the directly fixed value. For each time point 3-9 independent experiments were performed. Values are given as mean SEM.

\subsection{Isothermal titration calorimetry}

ITC was performed on a VP-ITC instrument (Microcal) at 25C. Samples were dialysed against degassed HEPES buffer (20 mM HEPES, pH 7.4, $150 \mathrm{mM} \mathrm{NaCl,} 1$ mM DTT). Titrations were carried out by $10 \mathrm{ml}$ injections. The stoichiometry was determined using a single-site model and measured heat released on binding was integrated and analysed with Microcal Origin 7.0 using a seqential-site binding model, yielding the equilibrium association constant $\mathrm{Ka}$, the enthalpy of binding $\mathrm{DH}$, and the stoichiometry $n$. 


\section{Chapter 4}

\section{Results}

\subsection{Basic In Vitro Characterization of the Disas- sembly Reaction}

\subsubsection{Purification of Active, Hexameric NSF}

In order to investigate the disassembly reaction on a molecular level, at first all proteins involved had to be purified. As opposed to the neuronal SNARE proteins and $\alpha$ SNAP, whose purification was already well established in the lab, NSF turned out to be a challenging protein to purify in its active state. The original idea was to purify the histidin-tagged NSF via Ni/NTA- followed by anion exchange chromatography and finally separate the different homo-oligomers from monomers and one another.

Unfortunately, even though it expresses well, is soluble, eagerly binds to anion exchange materials and nicely elutes at a defined $\mathrm{NaCl}$-concentration, it soon became evident that a major amount of activity is irreversibly lost during anion exchange chromatography rendering reproducible data collection impossible. Therefore, to optimize purification conditions, in addition to size- and purity-control via SDS-gel electrophoresis, NSF activity was tested after each step of purification using the FRET assay explained in more detail in section 4.1.4.

This led to the insight that Ni/NTA purification yielded best amounts of nondegraded NSF if pursued in presence of $\mathrm{Mg}^{2+} / \mathrm{ATP}$, a condition that allows for constitutive disassembly. A fast procedure and frequent buffer-exchange are mandatory to minimize degradation and retain activity, probably because constitutive ATPase activity can lead to limiting amounts of ATP, which have been described to cause NSF to fall apart into its monomers [8]. Monomers are presumably more accessible to bacterial proteinases than hexamers, and subsequent to longer purification procedures, bands at the size of approximately $25 \mathrm{kDa}$ and a $60 \mathrm{kDa}$ can be witnessed after SDS-PAGE. These bands coincide with those of the D1D2- and the N-terminal domain respectively.

Furthermore, anion exchange chromatography turned out to be inappropriate 
for purification of NSF. The anion exchange step would have been advantageous because as a positive side-effect of purifying it is also suited to concentrate the sample. Instead, pure and hexameric NSF had to be purified via gel filtration chromatography (Superdex200) after dialysis following the elution from the nickel beads. Best results were obtained when the presence of $\mathrm{Mg}^{2+}$ and hence constitutive ATPase activity was kept as short as possible and ongoing degradation could be prevented by immediate dialysis against a buffer containing ATP and EDTA after elution from the Ni-column. Since NSF precipitates at low salt levels, $\mathrm{NaCl}$ concentration during purification should be kept above $175 \mathrm{mM}$.

\subsubsection{Monitoring Disassembly Via SDS-PAGE}

An easy way to monitor the disassembly reaction exploits the fact that the ternary SNARE core complex is stable in SDS sample buffer, unless heated, and therefore appears as one single band after SDS gel electrophoresis [12]. Incubation of purified SNARE complex with NSF and $\alpha$ SNAP under disassembly conditions prior to gel electrophoresis will lead to SNARE-complex disassembly which can then be seen as a loss of intensity of the complex band and the appearance of additional bands representing the single SNARE proteins. This is illustrated in figure 4.1, where the kinetics of NSF disassembly are compared to that of a phosphomimetic mutant (NSFY83E) which has been reported to show a defect in $\alpha$ SNAP binding.

Even though this readout allows to differentiate between samples which show a dramatically different degree of disassembly, it is only semi-quantitative in the sense that the efficiency of disassembly can only be estimated by the intensity of the Coomassie stain on the gel. Secondly, the time resolution is too low to detect subtle differences. Furthermore, the reaction can not be monitored online, making the observation of reversible changes improbable. For example, differences in speeds of re-assembly between different SNARE targets, which can lead to apparently different amounts of disassembly, would presumably be misinterpreted to represent differences in disassembly. Hence, the next step was to establish a quantitative method with high time resolution which also allows to monitor the reaction online.

\subsubsection{Online Monitoring of Disassembly Via Fluorescence Spectroscopy}

Since fluorescence spectroscopy using fluorophore-labelled SNARE proteins is well established in the lab, the idea was to also monitor the disassembly reaction this way. Two types of experiments, exploiting different properties of fluorescence were set up. The first, FRET spectroscopy, makes use of the fact that energy transfer can take place between certain pairs of fluorophores, provided that the emission spectra of one (donor) overlaps with the excitation spectra of the other (acceptor). The amount of this so called fluorescence resonance energy transfer (FRET) is proportional to the inverse sixth power of the distance between the fluorophores, a property which 


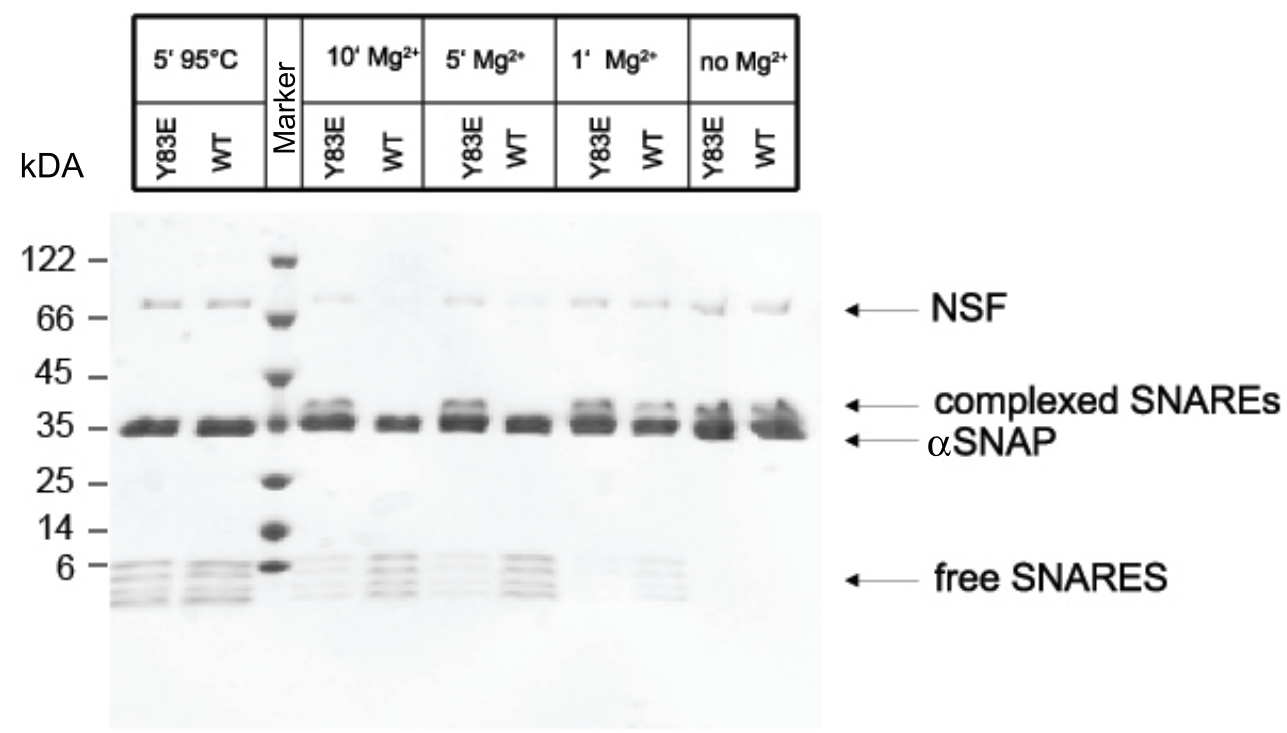

Figure 4.1: Monitoring disassembly on a gel. Purified 'mini'-complexes were incubated in buffer including $5 \mathrm{mM} \mathrm{Mg}^{2+} / \mathrm{ATP}, 35 \mu \mathrm{M} \alpha \mathrm{SNAP}$ and $1 \mu \mathrm{M}$ NSF or NSFY83E, respectively, for periods as indicated (1,5 or 10 minutes). As a comparison, one reaction mixture was heated to $95^{\circ} \mathrm{C}$ for 5 minutes to completely disassemble all complexes (first two lanes) prior to gel-electrophoresis. As a negative control one reaction did not contain $\mathrm{Mg}^{2+}$ (last two lanes).

allows for monitoring of protein interactions. If the fluorophores are attached to different, putatively interacting proteins under study, then upon interaction donor emission will decrease while acceptor emission will simultaneously increase.

The second, fluorescence anisotropy, depends on the influence of rotational motion on the fluorescence scattering of fluorophores in solution. It is proportional to the ratio of parallel to perpendicular scattered light with respect to the plane of the polarized excitation beam. Rotation of fluorophores shifts the plane of scattered emission and hence changes the anisotropy. Since small particles rotate faster than larger ones, the anisotropy can often be correlated to the size of the protein carrying the fluorophore, and conclusively serve as an indicator of protein binding or complex formation which slows down particle rotation.

To generate fluorescent substrates, maleimide-conjugated fluorophores can specifically be attached to the sulphur atom of cysteines via a covalent bondage. Various single-cysteine mutants of the neuronal SNARE proteins were already available (e.g. [60]), providing good candidates to function as suitable substrates during fluorescence based disassembly assays.

\subsubsection{The FRET Assay}

At first, to find a FRET pair suited for disassembly, FRET pairs known to lead to good signal changes during SNARE assembly were tested. An example is given 
in figure 4.2. Since here we are looking at complex dissociation, i.e. the distance between donor and acceptor increases, donor fluorescence will increase upon disassembly of the SNARE complex. Effectively, we therefore observe 'de-FRETTING' rather than 'FRETTING' during disassembly. For simplicity, only the donor spectra are shown in the following.

The SNARE complex used here was generated by incubation of stoichiometric amounts of SNAP25, the soluble part of Synaptobrevin and the H3 domain of Syntaxin1A. The donor dye (Oregon green) was attached to the 28th position of Synaptobrevin $\left(\mathrm{Sb}^{28 O G}\right)$ and the acceptor (Texas red) to the 130th position of SNAP25 (SNAP25130TR).
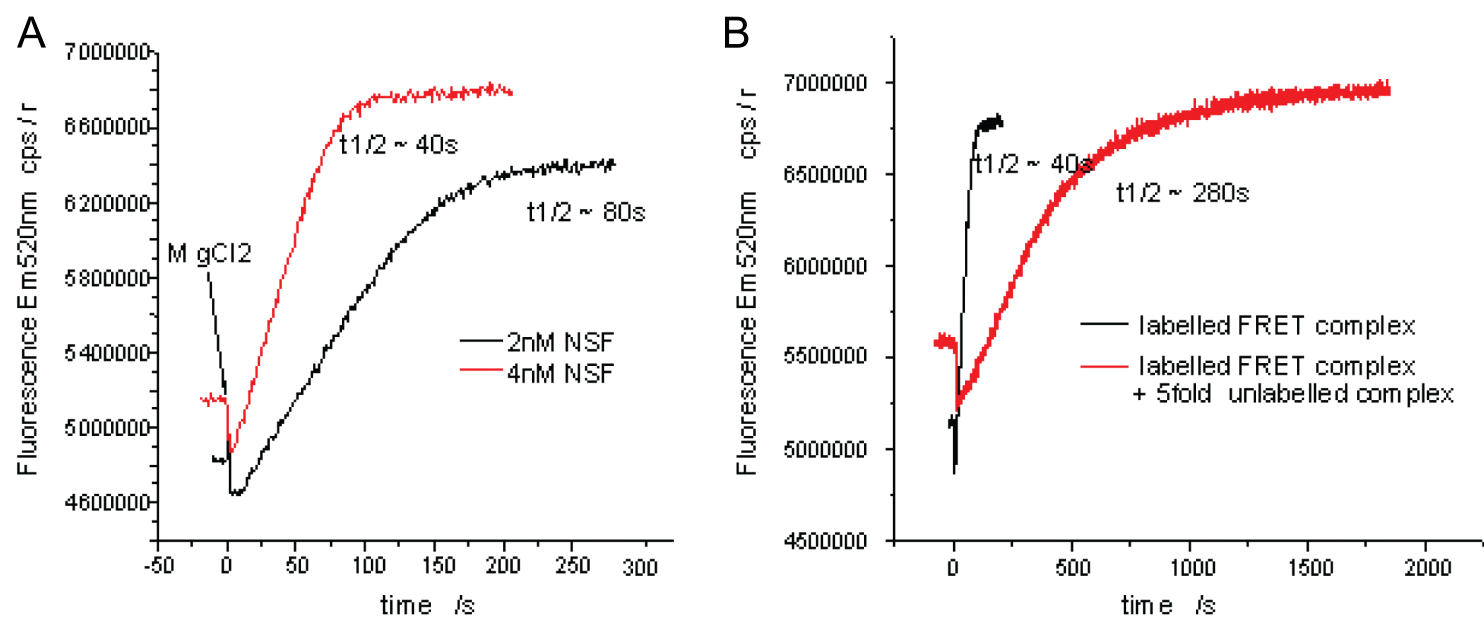

Figure 4.2: Monitoring disassembly of a core SNARE complex using FRET spectroscopy. Approximately 80nM of each FRET complex component (H3/SNAP25/Sb ${ }^{28 T R}$ ) were mixed with $1,8 \mu \mathrm{M}$ of $\alpha \mathrm{SNAP}, 2 \mathrm{mM}$ ATP and NSF as indicated, and the reaction was started by addition of $\mathrm{Mg}^{2+}$. (A) The half-time of the reaction correlates with the NSF concentration. (B) Addition of $\sim 400 \mathrm{nM}$ of unlabelled SNARE complex to the reaction results in a 5-fold decrease of apparent disassembly. This indicates that the labelled, 'visible' complex is disassembled at comparable speeds as the unlabelled (invisible) SNARE complex and hence disassembly is not impaired.

To ensure that the labelling of the SNARE complex does not influence disassembly kinetics of the wildtype SNARE complex, a five-fold excess of unlabelled SNARE complex was added to the reaction. Even though this unlabelled complex can not be observed in the spectrum, the apparent half-time of the labelled complex visible in the emission spectrum should increase by a factor of six if both complexes are disassembled equally well. The half-time indeed increased from 40 to 280 s, indicating that the labelled complex is disassembled at comparable speeds as the unlabelled and hence labelling does not influence disassembly. Knowing this, various buffering conditions were tested for optimal rates of disassembly, eventually leading to the conclusion that the reaction dramatically slows down in presence of $\mathrm{NaCl}$ or $\mathrm{KCl}$ concentrations higher than $120 \mathrm{mM}$, whereas a combination of KGlu/Kac at concen- 
trations of $120 \mathrm{mM}$ and $20 \mathrm{mM}$ respectively still allows for efficient disassembly. The chelating agent EDTA, which depletes the reaction of $\mathrm{Mg}^{2+}$, can be used to stop disassembly. If the concentration of free SNAREs is high enough at this point, reassembly takes place. Furthermore the reaction is inhibited by high amounts of ADP and can be irreversibly stopped by addition of $\mathrm{AlF}_{3}$, which supposedly freezes NSF in a transition state. Likewise, NEM (N-ethyl-maleimide) blocks the disassembly reaction in this assay.

\subsubsection{Fluorescence Anisotropy as a Readout for Disassem- bly}

As for the FRET assay, different labelling positions were tested for their suitability to serve as the target in fluorescence anisotropy measurements. Since here the readout depends on the flexibility of the dye, which may or may not change upon interaction with other proteins, it was less predictable, which positions would be useful and which would not. Furthermore, as opposed to the FRET assay, interaction of the dye with components of the disassembly machinery might also lead to anisotropy changes undifferentiable from those caused by SNARE interactions. Setting up an anisotropy measurement, therefore requires a close look at stepwise anisotropy changes after addition of each respective component to understand which signal changes are actually caused by which interaction. As an example, the spectra of a SNARE complex labelled with Oregon green at the 28th position of Syb2 ( $\left.\mathrm{Sb}^{28 O G}\right)$ during addition of $\alpha \mathrm{SNAP}$ and NSF as well as disassembly are shown in figure 4.3.

Note that adding $\alpha$ SNAP causes a significant increase of fluorescence anisotropy before the reaction is even started, which can also be seen when an $\alpha$ SNAP mutant known to be able to bind but not disassemble the SNARE complex, is added. The addition of NSF does not lead to a signal change, which is reasonable if one considers that enzymatic amounts of NSF are used. Upon triggering disassembly, the reaction including wt $\alpha \mathrm{SNAP}$, but not the one including mutant $\alpha \mathrm{SNAP}$, efficiently proceeds as can be told from a gradual decrease of fluorescence anisotropy.

\subsubsection{Optimizing Protein Amounts for Fast Disassembly Ki- netics}

Having optimized purification- and buffer-conditions which lead to best NSF performance in the in vitro fluorescence assays, I subsequently determined how much of the components of the disassembly machinery are needed for optimal disassembly. From the stoichiometry of $20 \mathrm{~S}$ complexes [61] one would assume that a molar ratio of NSF monomer to $\alpha$ SNAP to SNARE complex of 6:3:1 should be used for the disassembly reactions. Anyhow, considering that NSF is an enzyme, substoichiometric amounts for NSF are probably more reasonable in a functional assay. For NSF it should hence be possible to find a range in which the amount of enzyme correlates with functional efficiency in a linear fashion. Similarly, $\alpha$ SNAP could be sufficient 


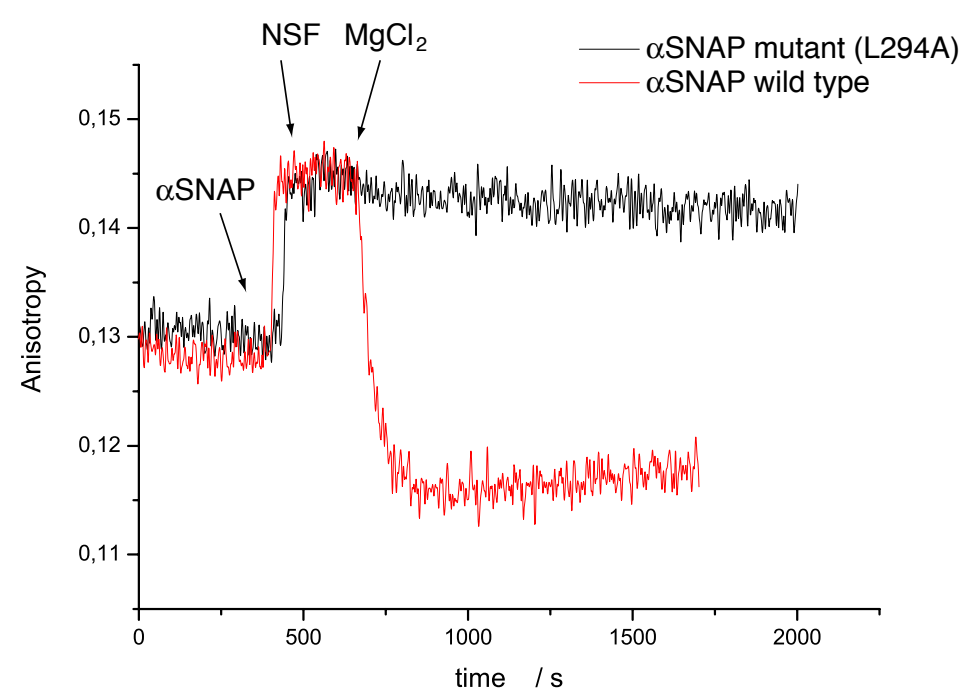

Figure 4.3: Monitoring $\alpha$ SNAP binding and disassembly of a purified core complex using fluorescence anisotropy. 150nM of purified SNARE complex labelled at Sb position $28\left(\mathrm{Sb}^{28 T R} / \mathrm{H} 3 / \mathrm{SNAP} 25\right)$ are disassembled in presence of $2 \mathrm{mM}$ ATP and $3,6 \mu \mathrm{M}$ of $\alpha \mathrm{SNAP}$. The addition of $1,2 \mu \mathrm{M} \alpha \mathrm{SNAP}$ or $\alpha \mathrm{SNAP}^{L 294 A}$, respectively, at the time point indicated leads to an increase of fluorescence anisotropy, whereas NSF addition at $\mathrm{t}=500 \mathrm{~s}$ does not significantly influence the spectrum. Finally, adding $\mathrm{MgCl}_{2}$ at $\mathrm{t}=540 \mathrm{~s}$ leads to SNARE disassembly in the $\alpha \mathrm{SNAP}$ wt-reaction, which can be observed as a gradual decrease of fluorescence anisotropy.

in enzymatic amounts, but might as well be required in a 3-fold excess with respect to the substrate, to saturate all complexes and thereby always keep the possible NSF acceptor-sites at a maximum. The reactions shown in figures 4.1.4 and 4.1.5 were conducted using excess amounts of ATP and $\alpha$ SNAP to ensure that NSF performance would not be underestimated due to limitations brought about by other components of the disassembly machinery. To open up possibilities to also employ the fluorescence assays for experiments investigating the reaction on the fuel- as well as the adaptor-level, it nevertheless is important to know as well, how much of these factors is required. Hence, NSF-, ATP- and $\alpha$ SNAP-dependence of SNARE disassembly were investigated under the as yet optimized buffer conditions.

\section{The enzyme - NSF dependence of core complex disassembly in solution}

In order to determine the concentration range of NSF at which it is able to efficiently disassemble SNARE complexes under the conditions used in the fluorescence assays, disassembly kinetics of approximately 130nM labelled SNARE complex using increasing amounts of NSF in presence of excess $\alpha \operatorname{SNAP}(3,6 \mu \mathrm{M})$ and ATP $(2 \mathrm{mM})$ were recorded. As shown in figure 4.4, as little as $1 \mathrm{nM}$ of NSF is sufficient 


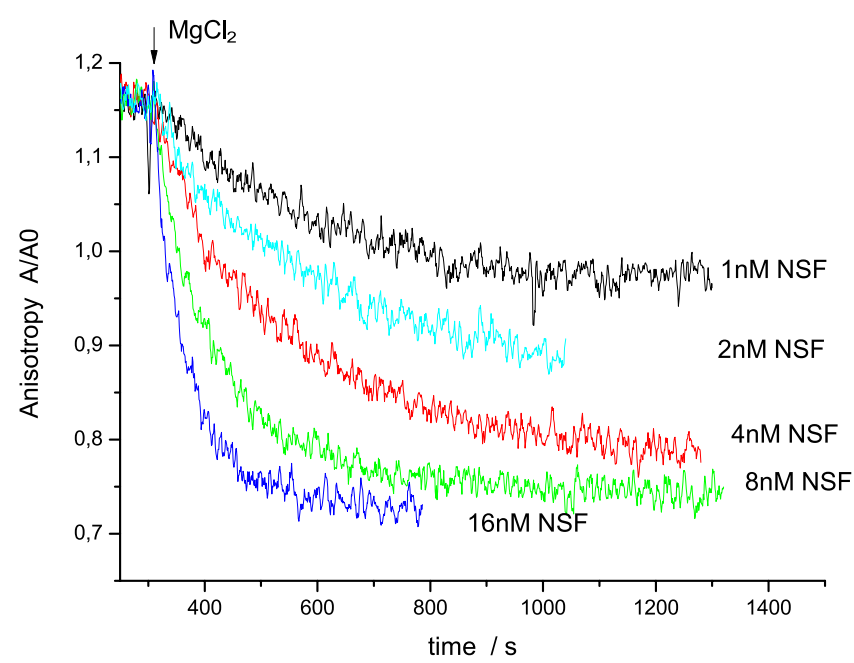

Figure 4.4: NSF dependence of core complex disassembly in solution. Disassembly kinetics of 130nM SNARE complex (H3/Sb28 $\left.{ }^{T R} / \mathrm{SNAP} 25\right)$ using increasing amounts of NSF in presence of excess $\alpha$ SNAP $(3,6 \mu \mathrm{M})$ and ATP $(2 \mathrm{mM})$ were recorded. The arrow indicates the start of the reaction by addition of $\mathrm{MgCl}_{2}$.

for optimal NSF performance. Up to $16 \mathrm{nM}$ enzyme, NSF-dependence is in a linear range, with speeds of disassembly of $\sim 15$ pmoles of SNARE complex per $\mu \mathrm{g}$ NSF per minute as calculated by the respective half-times. Due to ongoing re-assembly at later time points of the reaction caused by the increasing concentrations of free SNAREs, these rates are probably underestimates.

\section{The fuel - How much ATP?}

Subsequently, ATP-requirements for efficient SNARE disassembly under the conditions used in the fluorescence assay were investigated. To do so, disassembly kinetics of $75 \mathrm{nM}$ SNARE complex using increasing amounts of ATP in presence of excess $\alpha$ SNAP $(1,25 \mu \mathrm{M})$ and 10nM NSF were recorded. As shown in figure 4.5, the ATP concentration sufficient for optimal NSF performance is between 75 and $125 \mu \mathrm{M}$.

\section{The adaptor $-\alpha$ SNAP dependence of core complex disassembly in solu- tion.}

In order to determine the $\alpha \mathrm{SNAP}$ dependence under the conditions used in the fluorescence assays, disassembly experiments with $~ 90 \mathrm{nM}$ SNARE complex using increasing amounts of $\alpha$ SNAP in presence of excess ATP $(2 \mathrm{mM})$ and 10nM NSF were performed (figure 4.6). Notably, in line with published in vitro assays [62, 41], stoichiometric amounts of $\alpha \mathrm{SNAP}$ did not suffice to disassemble the SNARE 


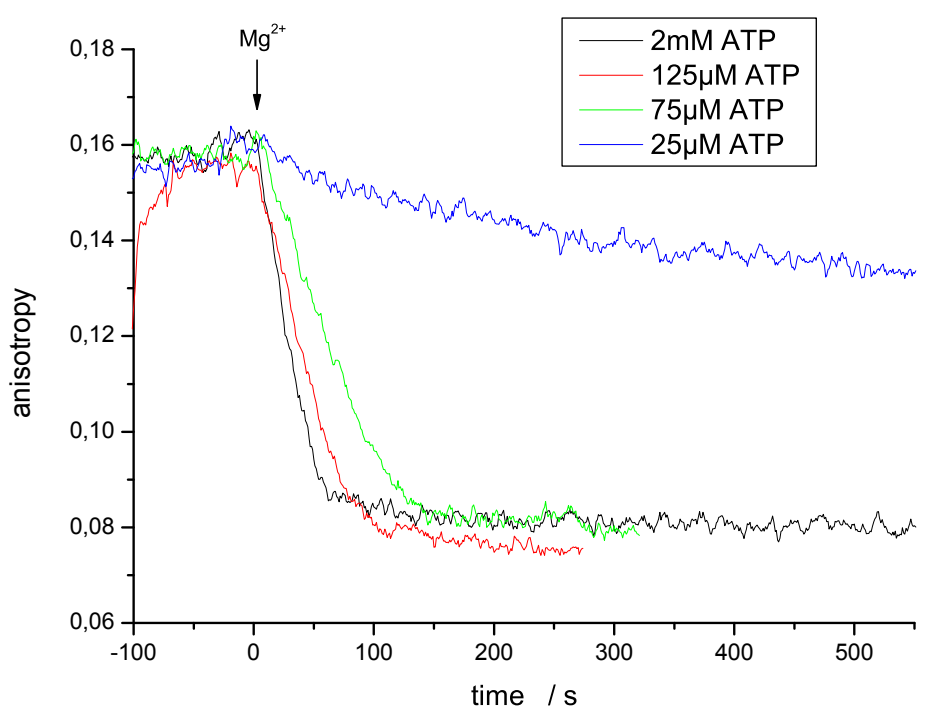

Figure 4.5: ATP dependence of the reaction.Disassembly kinetics of $\sim 75 \mathrm{nM}$ SNARE complex comparing increasing amounts of ATP in presence of excess $\alpha$ SNAP $(1,25 \mu \mathrm{M})$ and 10nM NSF were recorded. The arrow marks the start of the reaction by addition of $5 \mathrm{mM} \mathrm{MgCl}_{2}$.

complex. On the contrary, between $420 \mathrm{nM}$ and $1,25 \mu \mathrm{M}$ of $\alpha \mathrm{SNAP}$ are required for optimal speeds.

As will be seen in some later reactions, this may even be an underestimate due to the fact that at higher $\alpha$ SNAP concentrations the disassembly speeds reach the time resolution limit of the assay $(1,25 \mu \mathrm{M}$ and $3,75 \mu \mathrm{M}$ have half-times of $15 \mathrm{~s})$.

As mentioned in the introduction, these are not likely to represent physiological amounts, evoking the need to clarify the reasons for the apparent lack of $\alpha$ SNAP-efficiency before any quantitative comparisons on the adaptor-level can be conducted.

\subsection{How robust is the reaction?}

It as yet remains unclear, which parts of the SNARE complex are important for disassembly and, with respect to the assumed three sites of $\alpha$ SNAP/SNARE interaction and even six sites of NSF $/ \alpha$ SNAP interaction to which degree the machinery is robust in the sense that the specific interruption of single interaction sites only decreases reaction rates or leads to a complete abolishment of disassembly. If one found a way to abolish NSF/SNAP interaction by an alteration of either NSF or $\alpha$ SNAP, this would likely affect all SNAP/NSF interactions equally and consequently lead to a complete abolishment of SNARE disassembly. The interaction between SNAP and the SNARE-complex target however, is mediated by at least two and most likely 


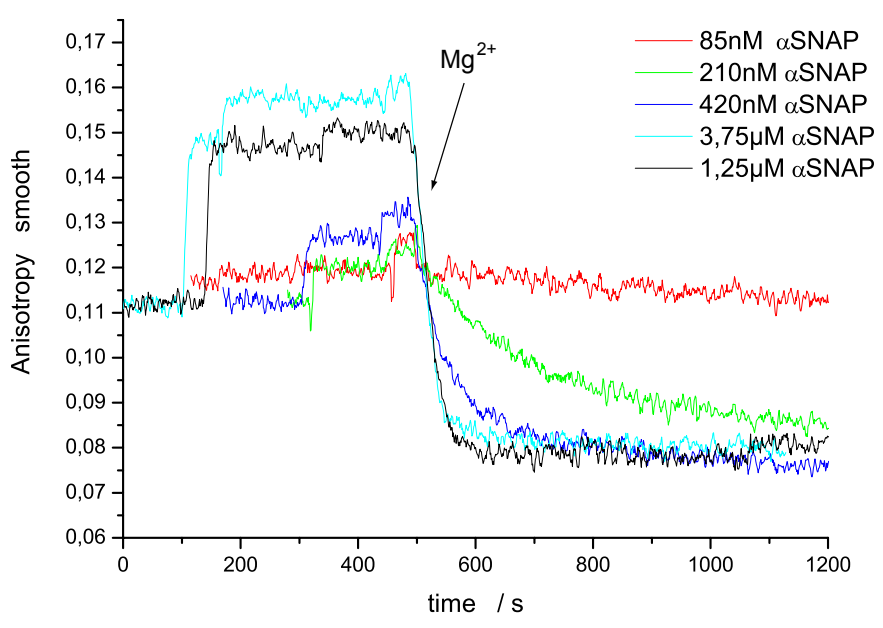

Figure 4.6: $\alpha$ SNAP dependence of core complex disassembly in solution. Disassembly kinetics of $75 \mathrm{nM}$ SNARE complex using increasing amounts of $\alpha$ SNAP in presence of $10 \mathrm{nM}$ NSF and excess of ATP $(2 \mathrm{mM})$ were recorded. The reaction was started at $\mathrm{t}=480 \mathrm{~s}$ by addition of $\mathrm{MgCl}_{2}$. Differences in fluorescence anisotropy prior to the start of the reaction result from different amounts of $\alpha$ SNAP bound to the complex (see figure 4.16). Note that more than $420 \mathrm{nM}$ of $\alpha \mathrm{SNAP}$ are needed for optimal disassembly.

three structurally different sites, whose 'loss-of-interaction' effects can be addressed independently. The minimal SNARE regions required for SNARE disassembly [63] as well as mutants which may or may not inhibit SNARE disassembly have been described $[64,62,65]$. In the following section, some of these findings are being re-investigated and additional SNARE-complex alterations assessed, exploiting the increased time resolution of the fluorescence assays as compared to the approaches used in the original publications.

\subsubsection{Inhibition of the Enzyme}

The N-terminal domain of NSF has been reported to be responsible for $\alpha$ SNAP binding [66]. An antibody against this domain might therefore abolish SNAP-binding and hence disassembly, if its epitope overlaps with the critical area.

\section{Generation of a functionally inhibitory antibody against the NSF N- terminal domain}

To generate an antibody which blocks NSF-function, the purified NSF N-terminal domain (residues 1-205) was used for immunization. After expression in E. Coli the protein was purified via Ni/NTA- followed by cation-exchange chromatography. The pure N-domain was then given to Michaela Hellwig who used it for immunization of rabbits and subsequently raised monoclonal antibodies from these. After selection, the supernatants of different clones were tested in the FRET assay, one of which 
turned out to block disassembly. This clone was then amplified and concentrated using a bioreactor and afterwards purified via ProteinA-sepharose by Michaela Hellwig. As shown in figure 4.7, 2,5 $\mu$ l of antibody after elution from the ProteinA column prevent 1,5nM of NSF from disassembling a soluble SNARE complex, which usually promote fast disassembly as shown in figure 4.4. Increasing the NSF concentration then allows to overcome the inhibition. The antibody could be shown to specifically recognize only NSF in rat brain cytosol as shown in figure 4.7 and none of the other proteins present in the disassembly assay. It can hence be concluded that binding of the anti-N antibody to NSF interferes with its function. 


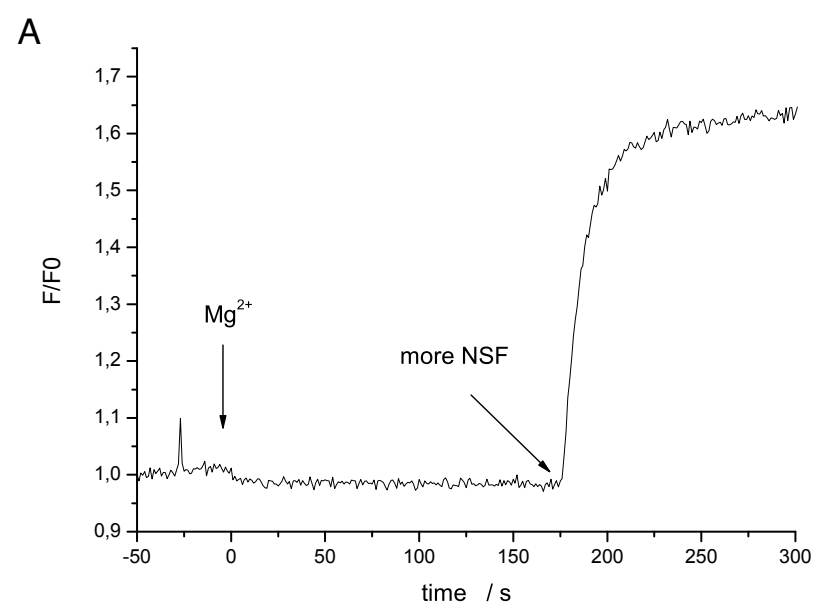

B
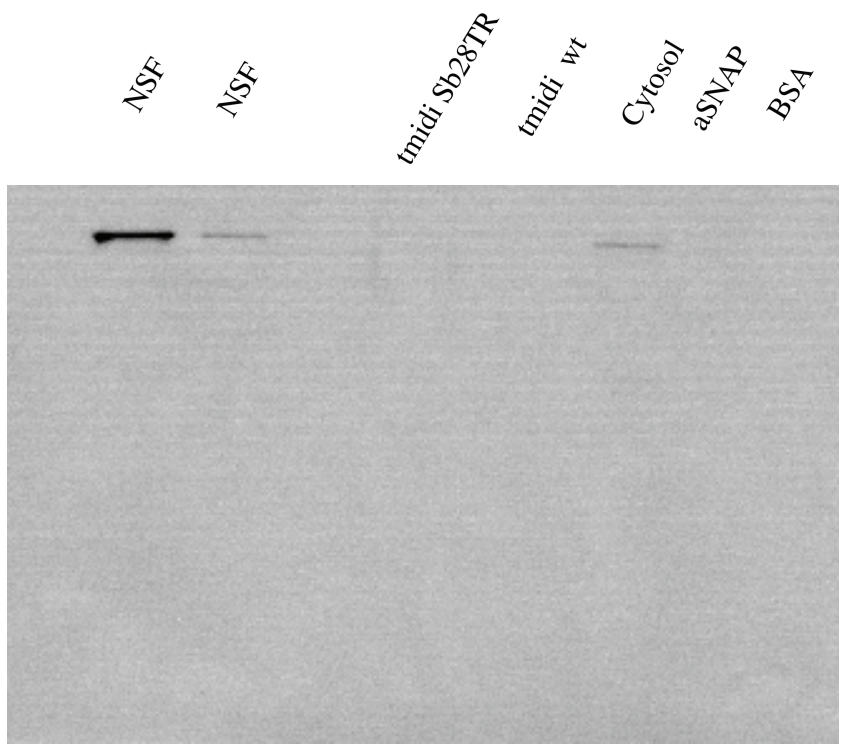

Figure 4.7: A monoclonal antibody directed against the NSF N-terminal domain inhibits SNARE-complex disassembly. (A) 2,5 $\mathrm{l}$ of anti-N domain antibody (clone 141-2) were pre-incubated with $3 \mathrm{nM}$ NSF and $\sim 40 \mathrm{nM}$ of FRET-SNARE complex (labels Syb ${ }^{28 T R}$ and $\mathrm{SNAP}^{130 O G}$ ) in reaction buffer. Subsequently, $1,5 \mu \mathrm{M}$ of $\alpha \mathrm{SNAP}$ were added and the reaction started by addition of $\mathrm{MgCl}_{2}$ at $\mathrm{t}=0$ seconds. Since no disassembly was observed, an additional $3 \mathrm{nM}$ of NSF were added after $175 \mathrm{~s}$, leading to the fast disassembly expected for 3nM NSF. (B) The indicated samples were blotted against clone 141-2 of the antibody to ensure specificity. 


\subsubsection{Influencing the Reaction on the Target Level}

\section{Full length soluble Syntaxin versus the Syntaxin H3 domain}

All the experiments described so far were performed with the full soluble domains of SNAP25 and Synaptobrevin-2, whereas of Syntaxin1A only the SNARE motif (H3 domain) was used. The large N-terminal domain of Syntaxin1A known to be involved in the regulation of SNARE-complex assembly, was deleted assuming that it does not influence disassembly kinetics. To prove this notion right, disassembly kinetics of a ternary complexes with either the full-length soluble portion or the H3 Domain of Syntaxin1A were compared. Complexes were assembled using equimolar amounts of SNAP25 $5^{130 T R}$, Synaptobrevin $280 G$ and Syntaxin or SyntaxinH3, respectively. After completion, disassembly was carried out as described above. Figure 4.8 illustrates that no major change in the disassembly rate was observed.

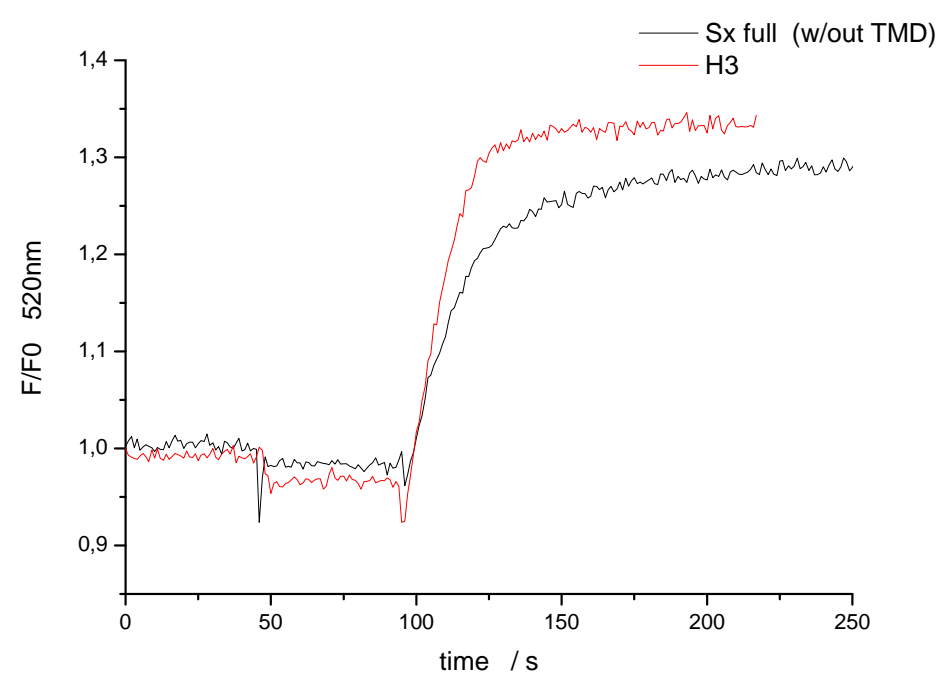

Figure 4.8: The Habc domain of Syntaxin1A is not required for SNARE complex disassembly. SNARE complexes either containing the full-length cytoplasmic part of Syntaxin1A or only the Syntaxin SNARE domain (H3) were subjected to disassembly by NSF and $\alpha$ SNAP as before. The reaction was started at $\mathrm{t}=90 \mathrm{~s}$, the time point at which the beginning of a the gradual increase can be observed in both graphs.

Various single or dual mutations in close vicinity impair SNARE disassembly only slightly, if at all

To elucidate whether certain regions of the SNARE complex are essential for disassembly, various SNARE complex mutants were to be investigated next. 


\section{Mutations in the SNARE-complex '0'-layer do not disturb SNARE dis- assembly}
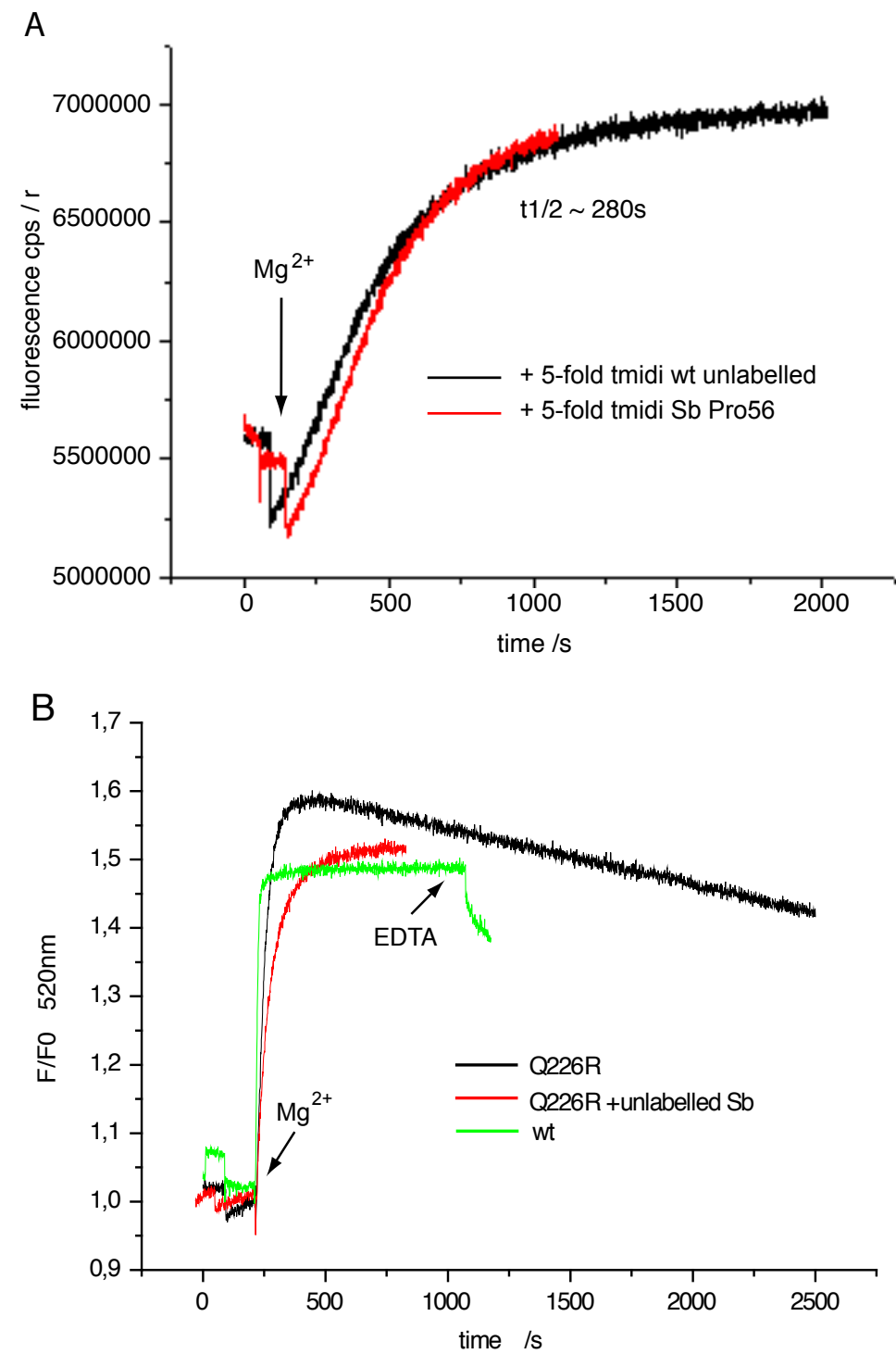

Figure 4.9: SNARE complexes with mutations in the '0'-layer are as prone to disassembly as wildtype complexes. (A) Pre-assembled FRET SNARE complexes (FRET pair $\mathrm{Sb}^{28 O G}$ and SNAP25 $5^{130 T R}$ (80nM of each component) were mixed with a five-fold excess of unlabelled purified complex containing either $\mathrm{Sb}^{\text {Pro56 }}$ or wildtype Sb. Subsequently $1,9 \mu \mathrm{M}$ $\alpha$ SNAP and $4 \mathrm{nM}$ NSF were added and finally $\mathrm{MgCl}_{2}$ at $\mathrm{t}=180 \mathrm{~s}$. (B) FRET-SNARE complexes assembled with either $\mathrm{H} 3$ or $\mathrm{H} 3^{Q 226 R}$ (FRET pair $\mathrm{Sb}^{28 O G}$ and SNAP25 ${ }^{130 T R}$, 80nM of each component) are disassembled by $1,9 \mu \mathrm{M} \alpha \mathrm{SNAP}$ and $4 \mathrm{nM}$ NSF. The sample represented by the red graph additionally contained $2 \mu \mathrm{M}$ unlabelled $\mathrm{Sb}$ to render re-assembly invisible. $\mathrm{MgCl}_{2}$ was used to trigger the reaction at $\mathrm{t}=220 \mathrm{~s}$. After 1100s EDTA was added to the wildtype reaction to show ongoing re-assembly. 
First, SNARE mutations in the '0'-layer were investigated. For Synaptobrevin this meant a mutation of the arginine residue 56 to proline $\left(\mathrm{Sb}^{R 56 P}\right)$, the '0'-layer glutamine ${ }^{226}$ of Syntaxin was mutated to arginine. In section 4.1.4, mixing unlabelled SNARE complex with $\mathrm{Sb}^{28 O G} / \mathrm{SNAP} 25^{130 T R} / \mathrm{H} 3$ FRET complex was used to demonstrate that the labelling does not impair disassembly. Now, the reaction from section 4.1.4 is shown in comparison to another one, which instead of wildtype complex included the same amount of $\mathrm{Sb}^{\text {Pro56 }}$-complex. Visible SNARE disassembly resulting from 'defretting' is as fast as for the wildtype reaction, indicating that both complexes are equally prone to disassembly and the $\mathrm{Sb}^{\text {Pro56 }}$ mutant conclusively does not impair disassembly.

To also re-investigate the Syntaxin '0'-layer mutant reported to cause a disassemblydefect in Scales et al. [64], H3 ${ }^{Q 226 R}$ was used for the second disassembly experiment illustrated in figure 4.9, where the disassembly kinetics of the mutation are compared to wildtype disassembly. Again, the reaction does not seem to be impaired, but what can be noted is some re-assembly very soon after disassembly is completed. This can not be seen in the wildtype reaction under these conditions, where EDTA needs to be applied to allow for visible re-assembly. In a third reaction, an excess of unlabelled $\mathrm{Sb}$ was added to the reaction to render re-assembly invisible. Here, the speed of disassembly seems slightly slower, which may be due to an increased invisible assembly-rate caused by the excess $\mathrm{Sb}$ which would increase target concentration and hence slow down the rate of visible disassembly towards the end of the reaction. Together, the slight kinetic differences observed do not support the notion that disassembly is changed significantly but might as well be explained by changes of one of the other interaction parameters, possibly an increased rate of re-assembly of the $\mathrm{Sx}^{Q 226 R}$-mutation. 


\section{C-terminal and layer-5 mutations of SNAP25 do not inhibit SNARE dis- assembly}

C-terminal deletions of SNAP25 and point mutations exchanging the two amino acids which SNAP25 contributes to the '5'-layer of the neuronal SNARE complex do not inhibit SNARE-complex disassembly. This was observed in experiments depicted in figure 4.10, which were carried out after the respective SNAP25 mutants were pre-assembled into complexes with a fluorescent dye attached to Synaptobrevin. In presence of $\alpha \mathrm{SNAP}, \mathrm{NSF}$ and ATP disassembly proceeds to the same extent at comparable speeds. To once more ensure that this is also true when compared to wildtype reactions labelled at a different position (which does not interfere with disassembly), the SNAP deletion mutants are shown in comparison to wildtype complex labelled with Texas red at Synaptobrevin ${ }^{\text {cys } 44}$. 
A

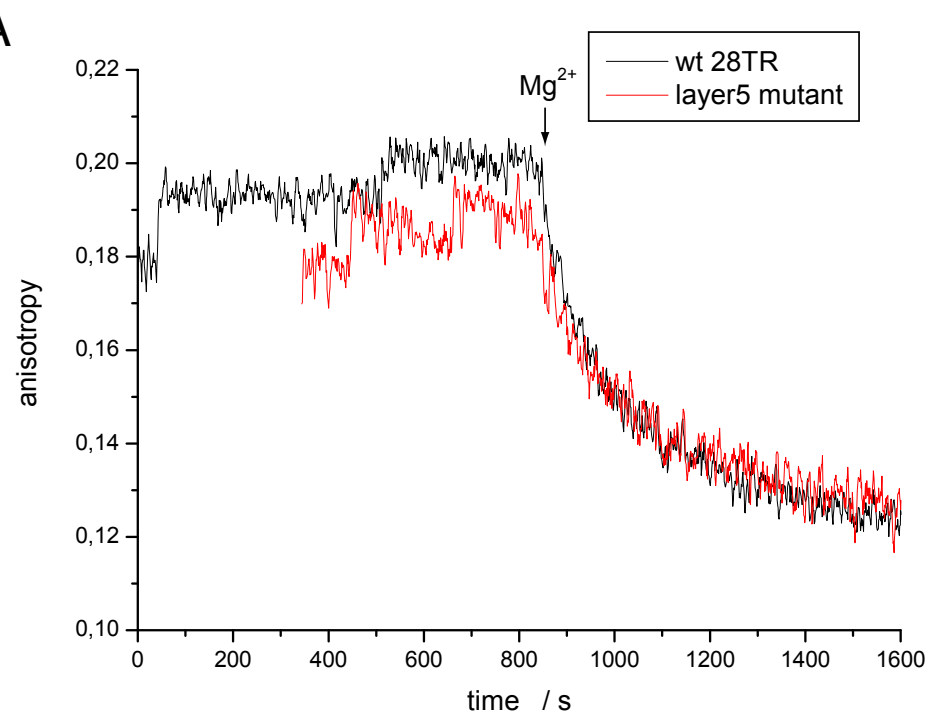

B

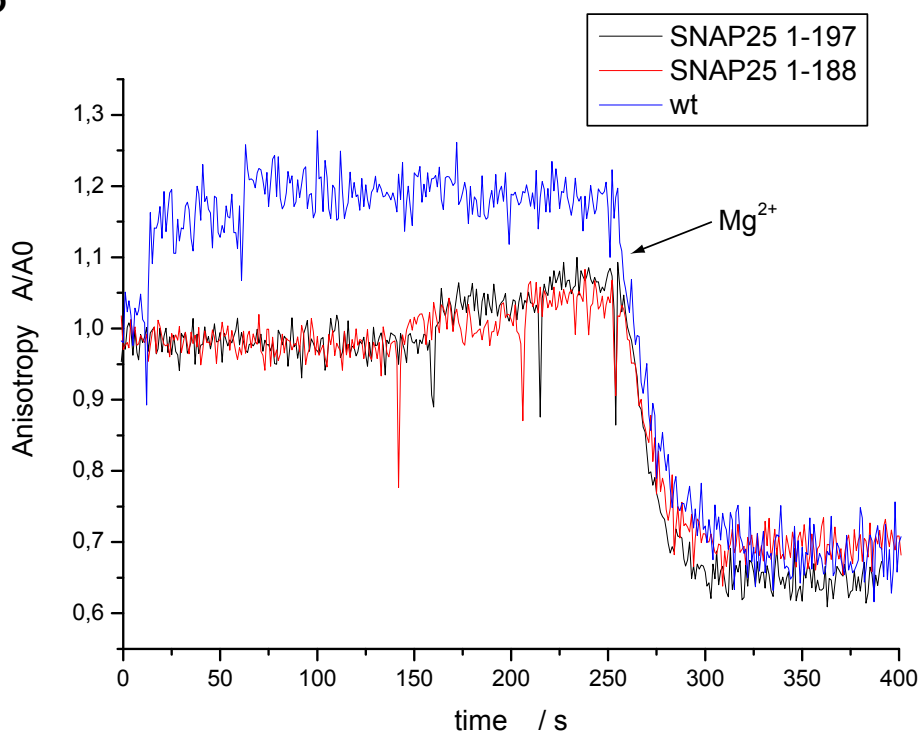

Figure 4.10: Neither the disassembly of complexes containing C-terminally deleted SNAP25 nor that of SNAP25 with two point mutations in the 5th hydrophobic layer is impaired. (A) Approximately 200nM of pre-assembled SNARE complex labelled at Syb $^{\text {cys } 28}$ containing either SNAP2 $5^{M 71 A, I 192 A}$ or wildtype SNAP25 were disassembled by $1,5 \mu \mathrm{M} \alpha \mathrm{SNAP}, 2 \mathrm{nM}$ of $\mathrm{NSF}$ and $2 \mathrm{mM}$ ATP. $\mathrm{MgCl}_{2}$ was used to trigger disassembly at $\mathrm{t}=850 \mathrm{~s}$. The reactions were performed in presence of soluble unlabelled Synaptobrevin $(2 \mu \mathrm{M})$ to prevent reformation of labelled complexes. (B) Disassembly of different pre-assembled SNAP25-mutant SNARE complexes $\left(\mathrm{Syb}^{28 T R} / \mathrm{H} 3 / \mathrm{SNAP} 25\right.$ mutant as indicated, 200nM of each component) compared to disassembly of wildtype complex ( $\mathrm{Syb}^{44 T R}$, $\mathrm{H} 3$ and SNAP25 wt). The reactions were performed in the presence of $1,6 \mu \mathrm{M} \alpha \mathrm{SNAP}$, $4 \mathrm{nM} \mathrm{NSF}$ and $2 \mathrm{mM}$ ATP and started by addition of $\mathrm{MgCl}_{2}$ at $\mathrm{t}=265 \mathrm{~s}$. All traces represent the changes in fluorescence anisotropy during the reaction. 


\section{C-terminal mutations of Sb, Sx or both do not or partially inhibit SNARE disassembly}

Next, C-terminal deletion mutants of SxH3 (183-240) and Sb(1-70) were scanned for disassembly defects. Sb deletion did not impair disassembly at all, whereas Sxdeletion led to a slight inhibition (see figure 4.11). The Sx mutant however did not reduce total amounts of disassembly, but only slightly impaired kinetics at the later stages of the reaction. Even when both, Sb and H3, were concertedly deleted, the inhibitory effect did not increase.

In summary, none of the various mutations examined in the last sections completely inhibited SNARE disassembly. Most deletions did not disturb disassembly at all. Partial impairment could only be seen when the C-terminal region of $\mathrm{H} 3$ was deleted, but even though the mutation slowed down disassembly kinetics towards the end of the reaction, all substrate present was sufficiently disassembled. Even the combination of two C-terminal SNARE deletion mutants (Sb and H3) did not increase the slight inhibition witnessed for $\mathrm{H} 3$ alone. One reason for this might be, that in all these cases only one SNAP/SNARE interaction site was disrupted. Hoping to finally abolish SNARE-complex disassembly completely, two non proximal regions were to be targeted simultaneously, as will be shown in the next section. 

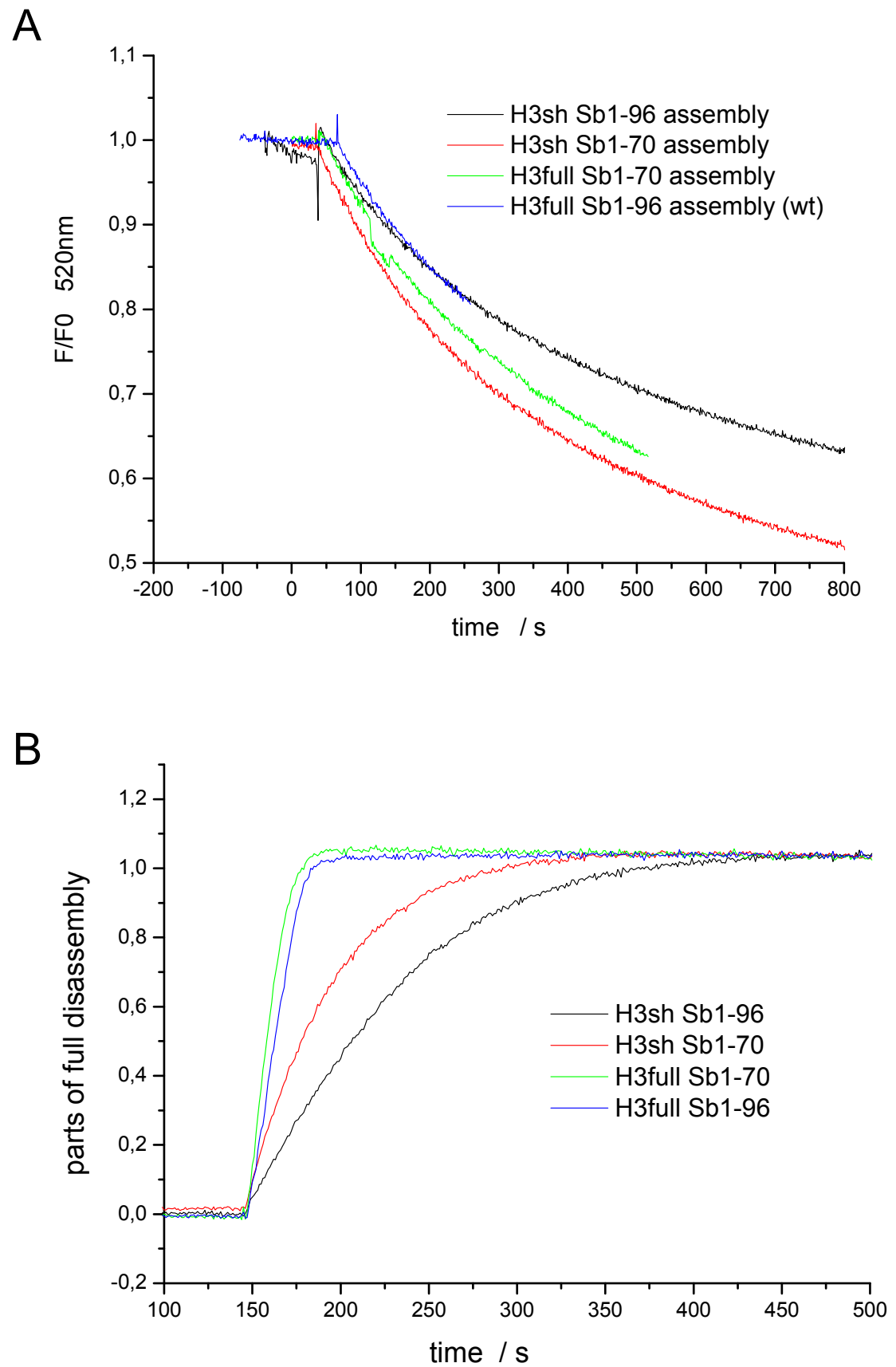

Figure 4.11: Disassembly of SNARE-complex deletion mutants. Different neuronal ternary FRET complexes, containing either an N-terminally shortened Synaptobrevin (1-70) or H3 (183-240) or both, were assembled (A) and subsequently disassembled in presence of $1,2 \mu \mathrm{M} \alpha \mathrm{SNAP}, 1,5 \mathrm{nM} \mathrm{NSF}$ and $2 \mathrm{mM}$ ATP by addition of $\mathrm{MgCl}_{2}$ at $\mathrm{t}=145 \mathrm{~s}$ (B). The 'parts of disassembly' are defined as the fraction of donor signal increase at a given point divided by the change of donor signal observed during full assembly of the com-

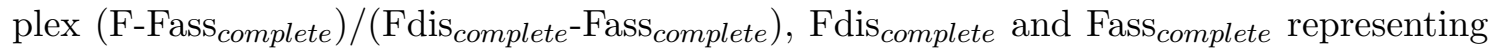
the donor fluorescence at complete disassembly or assembly, respectively. 


\subsubsection{Evidence for a Robust Target Recognition of the Dis- assembly Machinery}

\section{Inhibiting SNARE disassembly with the use of ternary complex-antibodies}

A different approach to investigate whether SNARE disassembly can be blocked on the target level, is not to delete parts of the complex but rather attach a factor, which either hides one or more interaction sites or sterically hinders conformational changes required for disassembly. Good candidates for proteins, whose attachment might influence function, are antibodies.

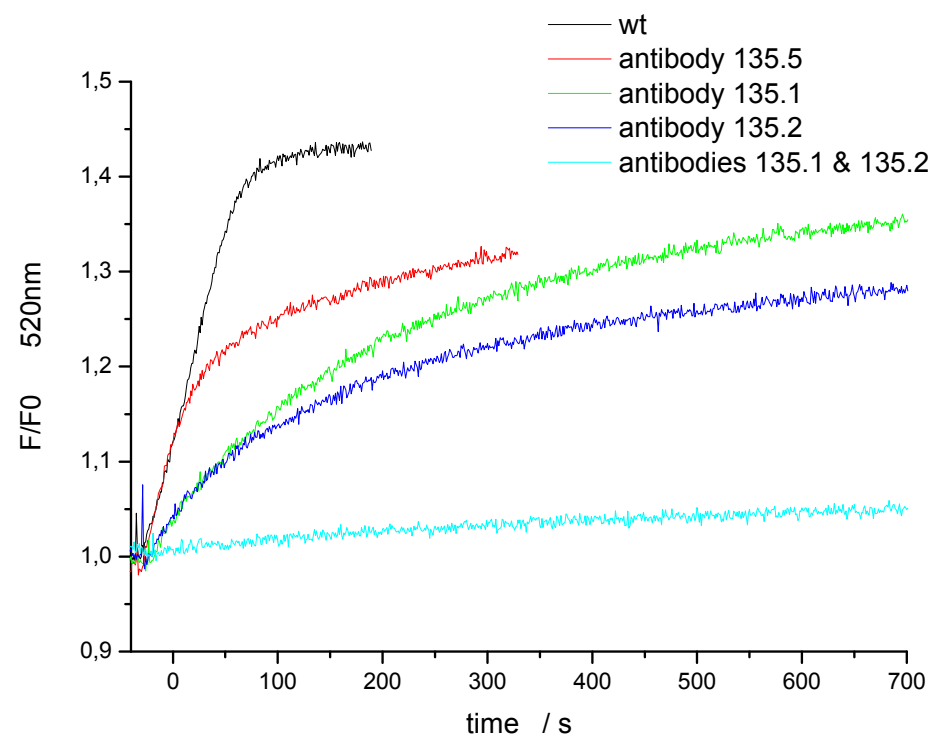

Figure 4.12: Two antibodies against the ternary SNARE complex act as synergistic inhibitors of SNARE disassembly. Approximately 70nM FRET-SNARE complex $\left(\mathrm{Sb}^{28 O G} / \mathrm{SNAP} 25^{130 T R} / \mathrm{H} 3\right)$ were incubated with the respective antibodies in disassembly buffer. Subsequently, NSF $(1,5 \mathrm{nM})$ and $\alpha$ SNAP $(1,2 \mu \mathrm{M})$ were added and the reaction started with $\mathrm{MgCl}_{2}$ at $\mathrm{t}=-40$ s.

Three different antibodies raised against the purified SNARE 'mini'-complex (Dirk Fasshauer \& Michaela Hellwig) had already been mapped to recognize different regions of the SNARE complex by Tabrez Siddiqui. ${ }^{1}$ Each of them alone as well as combinations of the antibodies were tested for their ability to inhibit SNARE disassembly using the FRET assay. As is shown in figure 4.12, all three antibodies partially impair disassembly, clones 131.1 and 131.2 having a slightly more pronounced effect than clone 131.5. Interestingly, if clones 131.1 and 131.2 are combined, they completely block disassembly. Considering that only half of the amounts used in the previous single-antibody experiments was applied in order to

\footnotetext{
${ }^{1}$ Tabrez Jamal Siddiqui, SNARE assembly and regulation on membranes, PhD thesis
} 
exclude a dosage dependent cause of the block, this finding indicates that the antibodies act synergistically, probably by interfering with the disassembly mechanism at two different sites of the complex.

\section{The combination of two non-inhibitory mutations can lead to inhibition of disassembly}

The last finding suggests that the NSF machinery might indeed be robust enough to function even though one of the interaction sites between substrate and machinery is weakened or blocked. Considering that the antibodies have been mapped to different regions of the complex, it is not likely that the two synergistic clones 131.1 and 131.2 interfere with the same site of interaction. This might indicate that even if one site is blocked, other sites might compensate for the defect and suffice to mediate disassembly. Similar to the observation made for the anti-complex antibodies, other structural alterations of the neuronal SNARE complex might also lead to inhibitions of function and maybe even show synergistic effects if they interfere with different sites of interaction. As will be shown in the next section, this synergy indeed turned out to be the case for two alterations, both of which did not exhibit any defect in disassembly on their own.

\section{Attachment of fluorescent dyes to $\mathrm{Sb}^{61}$ and $\mathrm{Sx}^{225}$ alone does not impair disassembly}

As pointed out in section 4.1.4, the FRET pair used throughout all experiments up to now $\left(\mathrm{Sb}^{28 O G} / \mathrm{SNAP} 25^{130 T R}\right)$ is equally prone to disassembly as wildtype ternary complex. This does not necessarily need to be true for all FRET pairs, since the attachment of labels at different positions might have a negative effect caused by steric hindrance or ablation of an important interface in any way. Hence, for every new FRET pair used, this possibility needs to be excluded first. Figure 4.13 therefore compares the speed of SNARE disassembly of a FRET pair which will be used in some of the following experiments $\left(\mathrm{Sb}^{61 O G} / \mathrm{H} 3^{225 T R}\right)$, to that of the one used hitherto. As can be seen, there is no significant difference between the disassemblykinetics of the two different FRET pairs.

\section{The FRET pair $\mathrm{Sb}^{61} /$ Syntaxin $^{225}$ combined with C-terminal SNAP25 deletions severely impairs disassembly}

Notwithstanding the fact that each of the two alterations alone discussed in sections 4.2.3 and 4.2.2 did not influence disassembly kinetics, the combination of both severely impairs disassembly as can be witnessed in figure 4.14. Here, the kinetics of SNAP25 C-terminally deleted SNARE complexes carrying the FRET pair $\mathrm{Sb}^{61 O G} / \mathrm{H}^{225 T R}$ are significantly slower than the wildtype reaction. Furthermore, of the SNAP25 $5^{\text {layer }}{ }_{\text {- }}$, SNAP25 $5^{1-197}$ - and SNAP25 $5^{1-188}$-complexes no more than $10 \%$, $20 \%$ or $30 \%$, respectively, are successfully disassembled, compared to $75 \%$ in the wildtype reaction. 


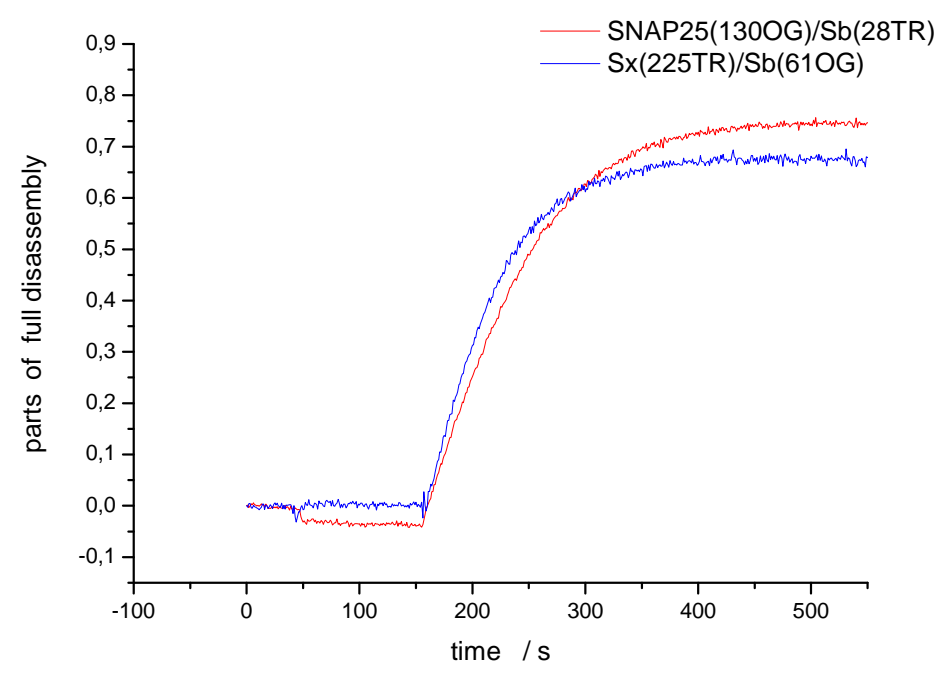

Figure 4.13: The two different FRET complexes $\mathrm{Sb}^{28 O G} / \mathrm{SNAP} 25^{130 T R} / \mathrm{H} 3$ and $\left.\mathrm{Sb}^{61 O G} / \mathrm{H}^{225 T R} / \mathrm{SNAP} 25\right)$ were disassembled in presence of $1,2 \mu \mathrm{M} \alpha \mathrm{SNAP}, 1,5 \mathrm{nM}$ NSF and $2 \mathrm{mM}$ ATP, triggered by addition of $\mathrm{MgCl}_{2}$ at $\mathrm{t}=160 \mathrm{~s}$. The parts of full disassembly are defined as the fraction of signal increase at a given point divided by the change of signal observed during full assembly of the complex (F-Fass complete $) /\left(\mathrm{Fdis}_{\text {complete }} \mathrm{Fass}_{\text {complete }}\right)$, Fdis complete $_{\text {and }}$ Fass $_{\text {complete }}$ representing the fluorescence at complete disassembly or assembly, respectively.

To exclude the possibility that the reactions with the SNAP25-mutations show a pronounced re-assembly, possibly due to slight inaccuracies with respect to the protein determination, the impact of an enhanced assembly and subsequent re-assembly was tested in further experiments, where the concentration of the respective SNAP25 was raised by a factor of four to promote faster assembly. As shown in figure 4.15, even though the acceleration has a subtle influence on disassembly, the differences between the mutant and the wildtype reaction remain much more prominent than those between the wildtype and the defective mutants. 
A

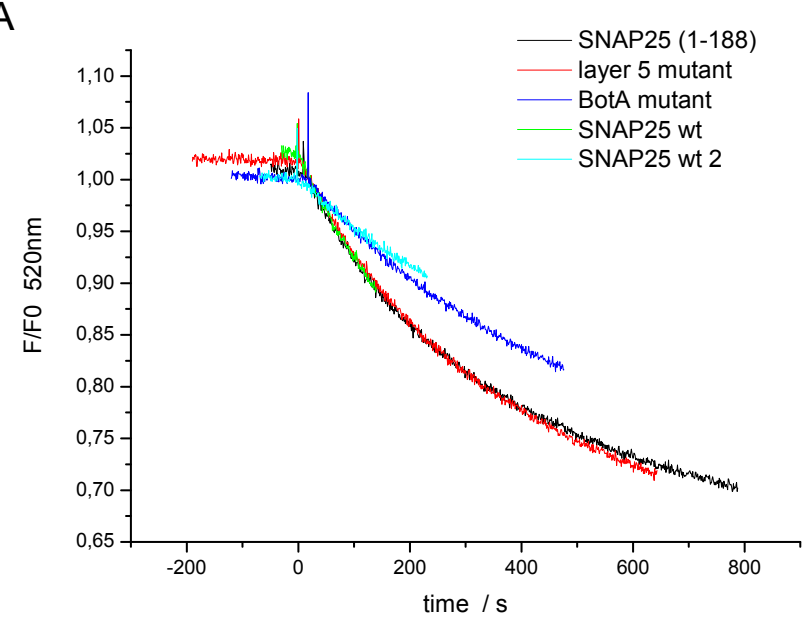

B

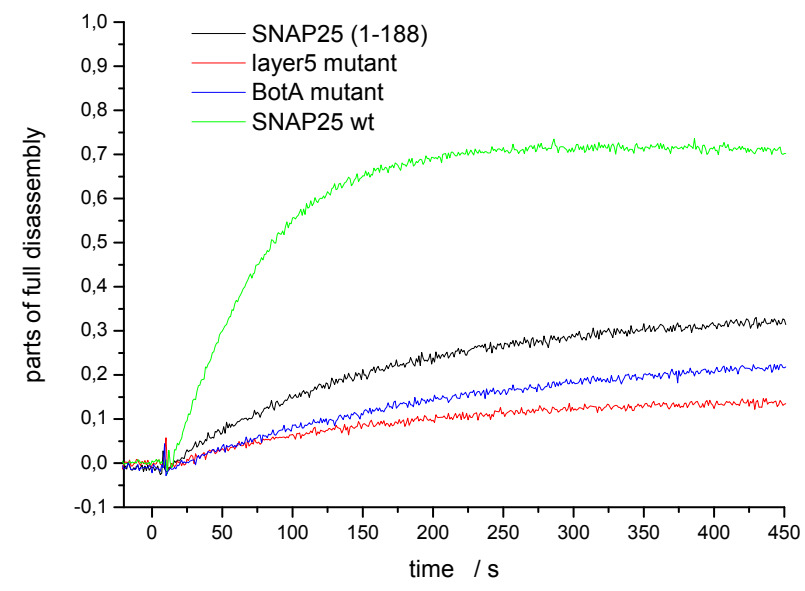

Figure 4.14: Two structural alterations of the SNARE complex show a pronounced synergy. Different SNAP25 mutants (BotA mutant: residues 1-197, layer- ${ }^{6} 5$ ' mutant: M71A/I192A) or wildtype SNAP were assembled with their cognate SNAREs to form complexes carrying the FRET pair $\left(\mathrm{Sb}^{61 O G} / \mathrm{H}^{225 T R}\right.$ introduced in section 4.2.3 (A) and subsequently disassembled in the presence of $1,2 \mu \mathrm{M} \alpha \mathrm{SNAP}, 1,5 \mathrm{nM} \mathrm{NSF}$ and $2 \mathrm{mM}$ ATP by addition of $\mathrm{MgCl}_{2}$ at $\mathrm{t}=0 \mathrm{~s}(\mathrm{~B})$. The 'parts of disassembly' are defined as the fraction of signal increase at a given point divided by the change of signal observed during full assembly of the complex (F-Fass complete $) /\left(\mathrm{Fdis}_{\text {complete }}-\mathrm{Fass}_{\text {complete }}\right), \mathrm{Fdis}_{\text {complete }}$ and Fass $\mathrm{Fmplete}_{\text {com }}$ representing the fluorescence at complete disassembly or assembly, respectively. 

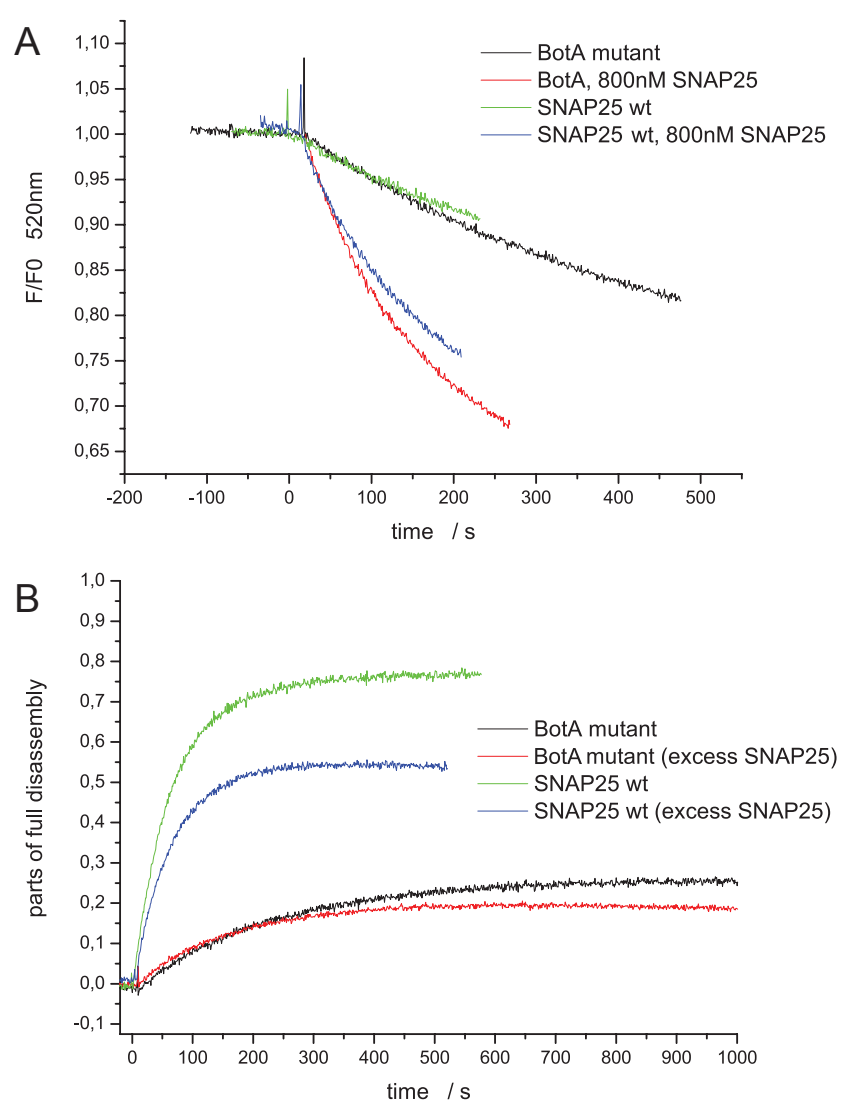

Figure 4.15: SNARE-complex assembly (A) and disassembly (B) were performed as in figure 4.14 except that a four-fold excess of SNAP25 $(800 \mathrm{nM})$ was used in two of the reactions to exclude ostensive differences in disassembly caused by slightly different reassembly kinetics.

\subsubsection{Inhibition on the SNAP Level?}

Having observed that certain structural changes on the substrate level can result not only in additive but even synergetic defects of disassembly, one may wonder how this can be explained on a mechanistic basis. One tempting speculation would be that these effects relate to the number of $\alpha$ SNAP bound to the complex during disassembly. Unfortunately, it is not possible to use stoichiometric - not to mention substoichiometric - amounts of $\alpha \mathrm{SNAP}$ at this stage, which would be needed to test this hypothesis, because as pointed out in section 4.1.6, optimal disassembly requires enormous amounts of $\alpha \mathrm{SNAP}$ in the fluorescence assays. It was therefore substantial to figure out first, how the efficiency of $\alpha$ SNAP could somehow be improved in our assays. 


\subsection{Why So Much $\alpha$ SNAP?}

In order to finally be able to address questions related to $\alpha$ SNAP action, the following sections will try to shed light on why the $\alpha$ SNAP-performance is so unexpectedly inefficient in the fluorescence assays and how this nuisance can be resolved. In principle the low $\alpha$ SNAP efficiency could be inherent to the recombinant $\alpha$ SNAP, possibly due to invisible degradation or a lack of posttranslational modifications in vivo, which do not occur when expressed in E. coli. Alternatively, it might be caused by non-optimal conditions in the fluorescence assays. To test, whether the recombinant $\alpha$ SNAP shows any intrinsic defects, different methods were employed.
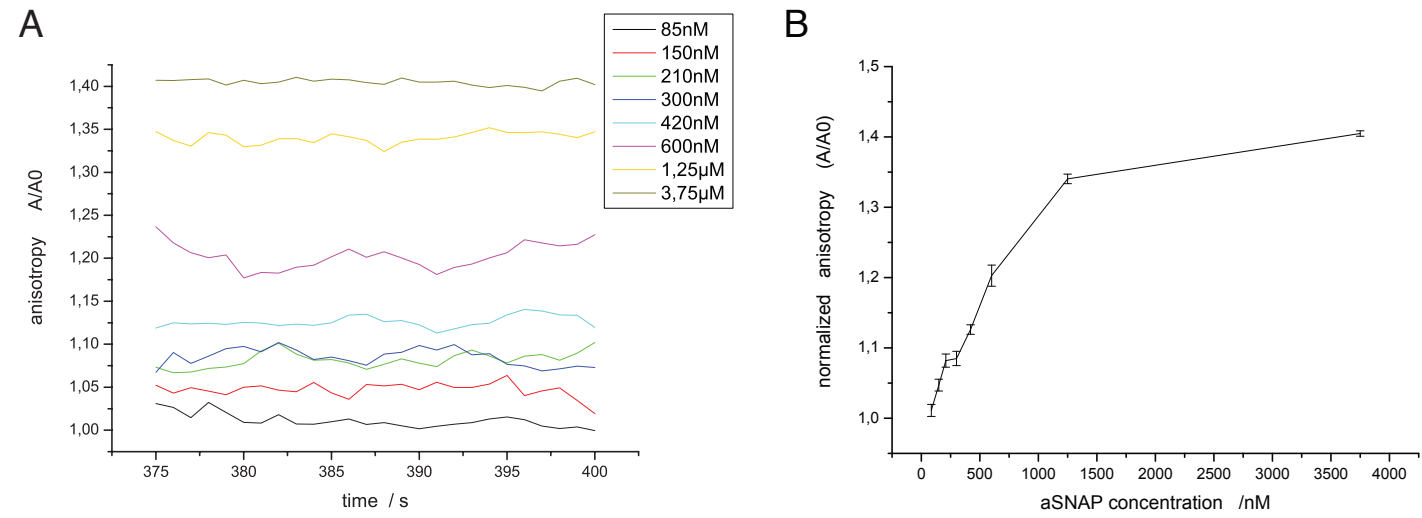

Figure 4.16: $\alpha$ SNAP binding to core complexes in solution. (A) Signal changes upon binding of increasing amounts of $\alpha$ SNAP to $\sim 75 \mathrm{nM}$ SNARE complex were recorded and are shown as multiples of the anisotropy before $\alpha$ SNAP addition. Note that even at $1,25 \mu \mathrm{M}$ of $\alpha \mathrm{SNAP}$, saturation is not reached. (B) Mean values of the graphs on the left are plotted against the $\alpha$ SNAP concentration.

As outlined above, fluorescence anisotropy also allows to monitor $\alpha$ SNAP-binding. Figure 4.16 illustrates that for $\alpha \mathrm{SNAP}$ binding the amounts needed to saturate the system are extremely high. Even at $1,25 \mu \mathrm{M} \alpha \mathrm{SNAP}$ the maximum signal change caused by $\alpha$ SNAP binding is not reached. The binding capacity saturates somewhere between $1,25 \mu \mathrm{M}$ and $3,75 \mu \mathrm{M}$ of added $\alpha \mathrm{SNAP}$ (here relating to a ratio of approximately seventeen $\alpha$ SNAPs to one complex and fifty $\alpha$ SNAPs to one complex, respectively).

\subsection{1 $\alpha$ SNAP Binding to the Complex Monitored Calori- metrically}

The fluorescence experiments in section 4.1.6, investigating $\alpha$ SNAP-binding, indicated that more than $17 \alpha$ SNAPs per complex were needed to achieve saturation. This finding dramatically differs from the 3:1 ratio one would expect and might be explained either by a reduced affinity or efficiency or alternatively by degradation, which renders a large portion $\alpha$ SNAP incompetent with respect to SNARE-complex binding. To check, whether the recombinant $\alpha$ SNAP binds the SNARE complex in 
a stoichiometric ratio of 3:1 when used at higher concentrations, isothermal titration calorimetry was performed. The calorimeter detects temperature changes with such high sensitivity that it can be used to quantify the small amounts of heat released or consumed during protein/protein interactions. One protein is titrated into the other in distinct steps in an adiabatic jacket, and enthalpy changes as well as the stoichiometric ratio can be directly measured.

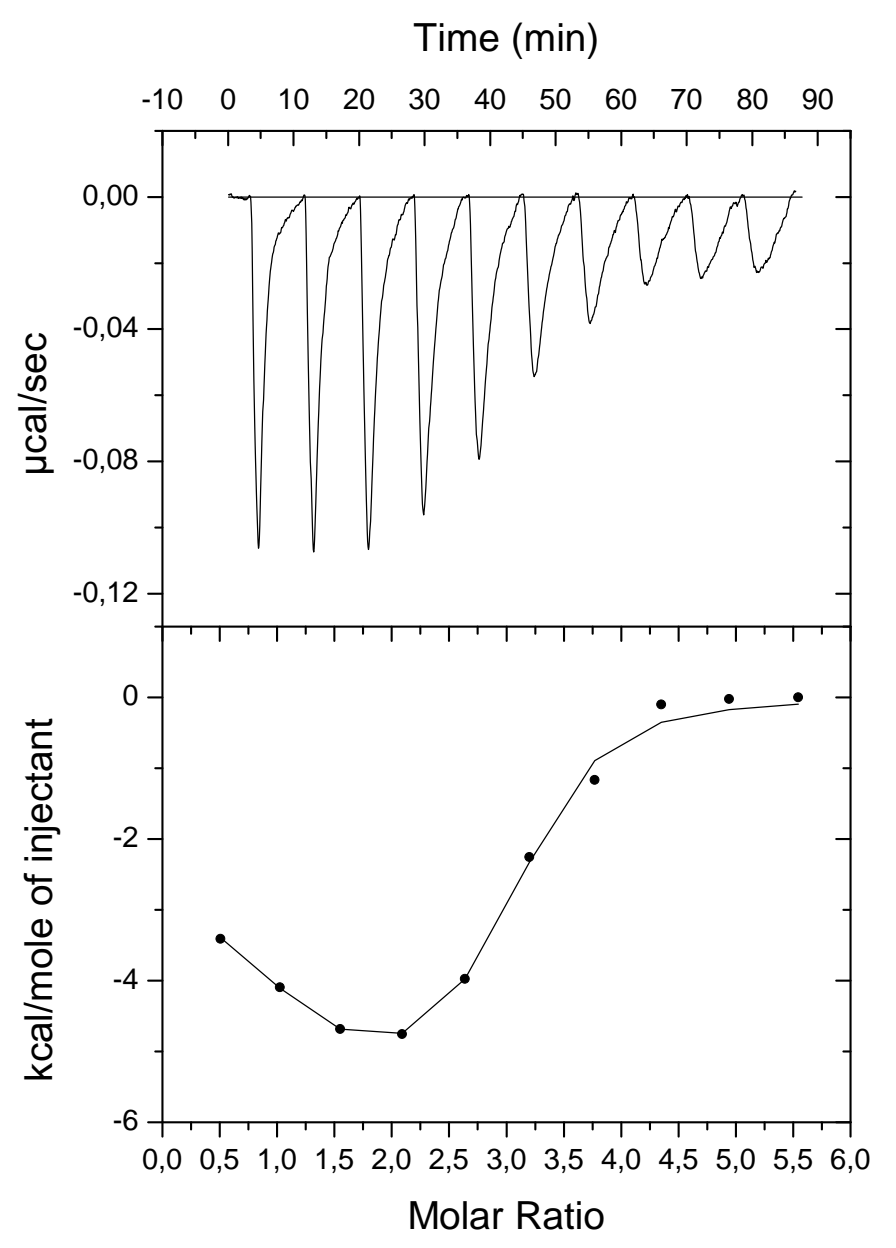

Figure 4.17: Isothermal titration calorimetry of $\alpha$ SNAP binding to the core complex. $\alpha \operatorname{SNAP}(70 \mu \mathrm{M})$ in the syringe was titrated in ten steps into purified ternary SNARE complex (H3/SNAP25/Sb, 2,5 $\mu \mathrm{M})$ in the cell. Shown are the enthalpy changes during the experiment on top, and the integrated areas normalized to the amount of $\alpha$ SNAP (kcal/mol) versus the molar ratio of $\alpha$ SNAP to the SNARE complex below. The solid line represents the best fit to the data for a three site sequential binding site model using a nonlinear least squares fit.

As can be seen in figure 4.17, the enthalpy changes are very low obscuring the possibilities to extract much thermodynamical information. The stoichiometry 'n' 
of the SNARE complex could nevertheless be determined to be $3 \alpha$ SNAPs per complex in these experiments. Notably, this is exactly what is expected and hence allows us to conclude, that the majority of the recombinant $\alpha \mathrm{SNAP}$ is able to bind SNARE complex, excluding the possibility that degradation is the reason for the low potency. Notwithstanding the low enthalpy values of the reaction, I still tried to fit the reaction in order to roughly estimate the $\alpha$ SNAP affinity. The thermodynamic data could not be properly fitted using a single site binding model, which assumes that $\alpha$ SNAP has the same affinity to all SNARE binding sites. Using a three-site sequential fit however, the curve could nicely be fitted (see figure 4.17). This suggests, that $\alpha$ SNAP binding to SNARE complexes is not equal to all three SNAP/SNARE interaction sites but the sites rather display different affinities. The affinities resulting from this fit were $10 \mathrm{nM}$ for the first, $50 \mathrm{nM}$ for the second and 380nM for the third binding site. Nevertheless, keeping in mind the low enthalpies and also considering that only a low number of data points per binding site were recorded, the affinities of $\alpha$ SNAP to the SNARE complex determined might not be very reliable.

\subsection{2 $\alpha$ SNAP Dependence of SNARE Disassembly on 'Mem- brane Sheets'}

Having established that $\alpha$ SNAP binds the SNARE complex at the expected ratio of 3:1, two possibilities remain: Either does the recombinant $\alpha$ SNAP have a reduced affinity as opposed to the endogenous protein, or a factor important for binding is missing in our fluorescence assay. Hence, the next step was to test the recombinant $\alpha$ SNAP's efficacy under the more physiological conditions of a different system, the so called membrane sheets. These membrane sheet experiments experiments were conducted together with Dana Bar-On.

Membrane sheets are generated by subjecting cultured, adherent PC12 cells to a gentle ultrapulse. The upper half of the cells is consequently ripped of, leaving behind an intact native membrane bilayer attached to a cover slip. The preparation provides the full set of membrane lipids and proteins in their native environment, including steric and conformational restriction, membrane fluidity, cytoskeletal components, lipid component diversity, and associated regulatory factors. 

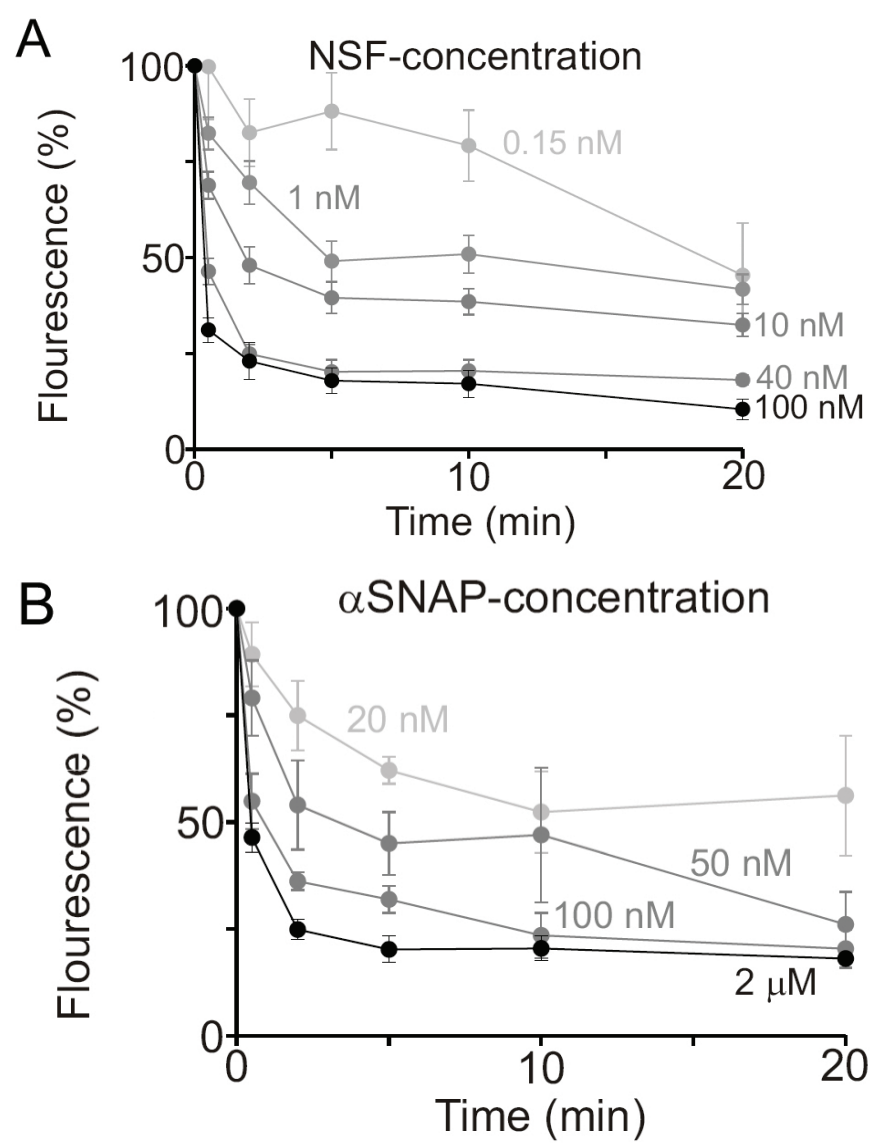

Figure 4.18: Disassembly of SNARE complexes on membrane sheets. After completion of complex formation, the membranes were incubated with increasing concentrations of NSF and $\alpha \mathrm{SNAP}$ in the presence of $\mathrm{Mg}^{2+} / \mathrm{ATP}$, allowing for disassembly of the formed SNARE complexes. Upon disassembly the soluble fluorescent synaptobrevin is released into the buffer making the observed loss of fluorescence from membrane sheets a direct read-out of the disassembly reaction (100\% represent total fluorescence before disassembly).

Cis-SNARE complexes in this experiment were generated by pre-incubation of membrane sheets with the fluorescent recombinant Synaptobrevin2 also used in the fluorescence assays. As in solution, the Synaptobrevin spontaneously assembles with its cognate t-SNAREs, here the endogenous Syntaxin and SNAP25 sitting on the PC12-cell membrane. After completion of complex formation, the membranes were incubated with increasing concentrations of NSF and $\alpha$ SNAP in the presence of $\mathrm{Mg}^{2+} / \operatorname{ATP}(5 \mathrm{mM})$, allowing for disassembly of the formed SNARE complexes. Upon disassembly, the soluble fluorescent synaptobrevin is released into the buffer making the observed loss of fluorescence from membrane sheets a direct read-out of the disassembly reaction. As illustrated in figure 4.18, fluorescence decreases with time in an NSF- and $\alpha$ SNAP-concentration dependent manner.

Surprisingly, under the conditions of the membrane sheet assay $\sim 100 \mathrm{nM}$ of $\alpha \mathrm{SNAP}$ are sufficient to mediate disassembly equally well as $2 \mu \mathrm{M}$ indicating saturation at $100 \mathrm{nM}$. Of course the amount of SNARE target in these assays is not 
known, not permitting a conclusion about the stoichiometry of $\alpha$ SNAP and SNARE complexes in this assay. Nevertheless, together with the ITC measurements which indicated a molar ratio of 3:1, these findings strongly suggest that a factor missing in the FRET and anisotropy experiments but present on membrane sheets is responsible for the higher $\alpha$ SNAP affinity. 


\subsubsection{Disassembly of SNARE Complexes Incorporated into Liposomes}

Having excluded that the recombinant $\alpha$ SNAP differs from the endogenous in a way that causes the low SNAP efficiency, the reason for the low power in the fluorescence assays was to be searched elsewhere. Since the same recombinant NSF and also identical buffer conditions to the ones used in the fluorescent assays were used in the membrane sheet assay, these two factors could also be neglected. Presumably, an additional factor or domain potentiating the $\alpha$ SNAP function had to exist, which was present in the membrane-sheet assay but as yet not in the cuvette.

Possible candidates were the SNARE transmembrane domains as well as the plasma membrane itself. To check, whether the presence of either of these improves $\alpha$ SNAP efficiency, SNARE complexes were incorporated into liposomes prior to $\alpha \mathrm{SNAP} / \mathrm{NSF}$ mediated dissociation. And indeed, in the presence of liposomes $300 \mathrm{nM}$ of $\alpha \mathrm{SNAP}$ suddenly sufficed to disassemble with the same kinetics as $1,5 \mu \mathrm{M}$ in solution in the FRET as well as the anisotropy assay (figure 4.19). Furthermore, it could be excluded that this was only due to low amounts of SNARE complex incorporated into the liposomes, because doubling the amount of complex as well as the amount of NSF still did not necessitate an increased $\alpha$ SNAP-concentration to finish disassembly in the same time-period as before. When disassembling liposomal complexes, $\alpha \mathrm{SNAP}$ is hence no longer limiting at $300 \mathrm{nM}$. 
A

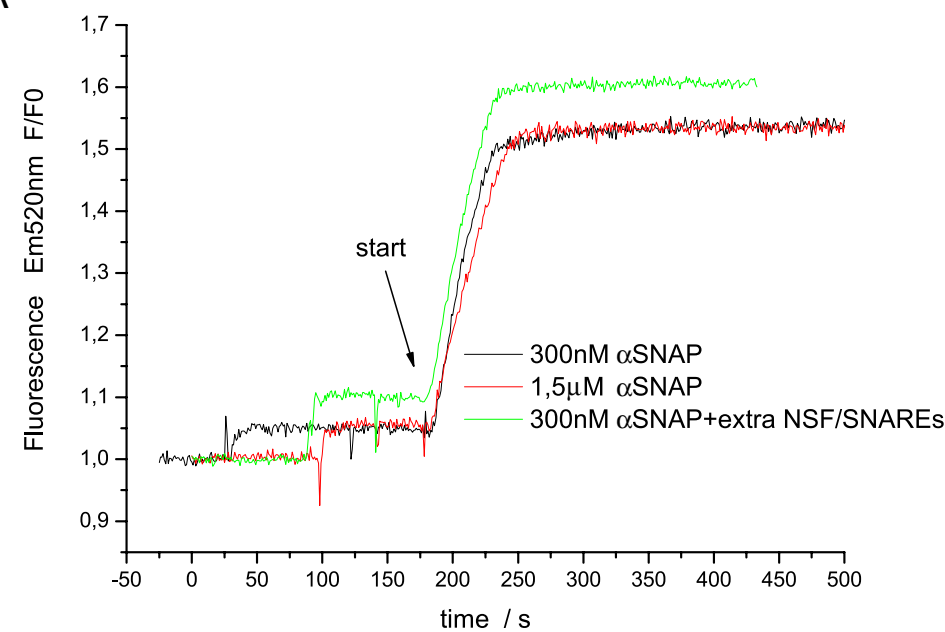

B

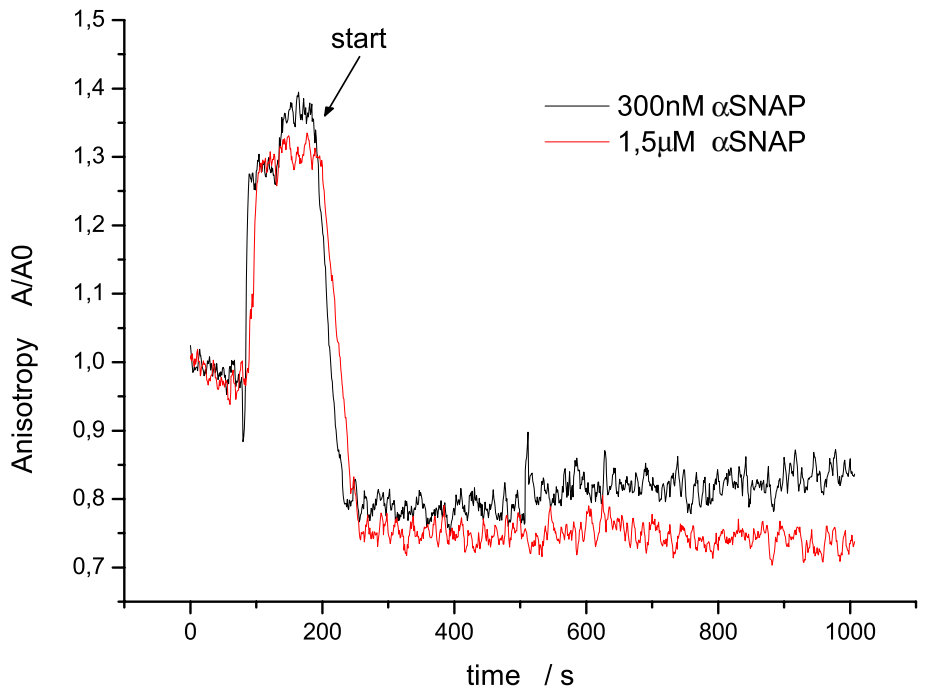

Figure 4.19: Liposomal SNARE complexes increase $\alpha$ SNAP efficacy. FRET spectroscopy (A) and fluorescence anisotropy (B) measurements of complexes incorporated into liposomes. Disassembly kinetics of SNARE complexes $\left(\mathrm{Sb}(1-116)^{T R 28} / \mathrm{H} 3 / \mathrm{SNAP} 25^{130 O G}\right.$ and $\mathrm{Sb}(1-116)^{T R 28} / \mathrm{H} 3 / \mathrm{SNAP} 25$, respectively) incorporated into liposomes were recorded. The reactions contained the $\alpha$ SNAP concentration as indicated, $6 \mathrm{nM}$ of NSF and $35 \mathrm{nM}$ complex. A third reaction shown in (A) also contained $300 \mathrm{nM}$ of $\alpha \mathrm{SNAP}$ but $12 \mathrm{nM} \mathrm{NSF}$ and $70 \mathrm{nM}$ complex. All reactions were started at $\mathrm{t}=190 \mathrm{~s}$ by addition of $\mathrm{MgCl}_{2}$. 

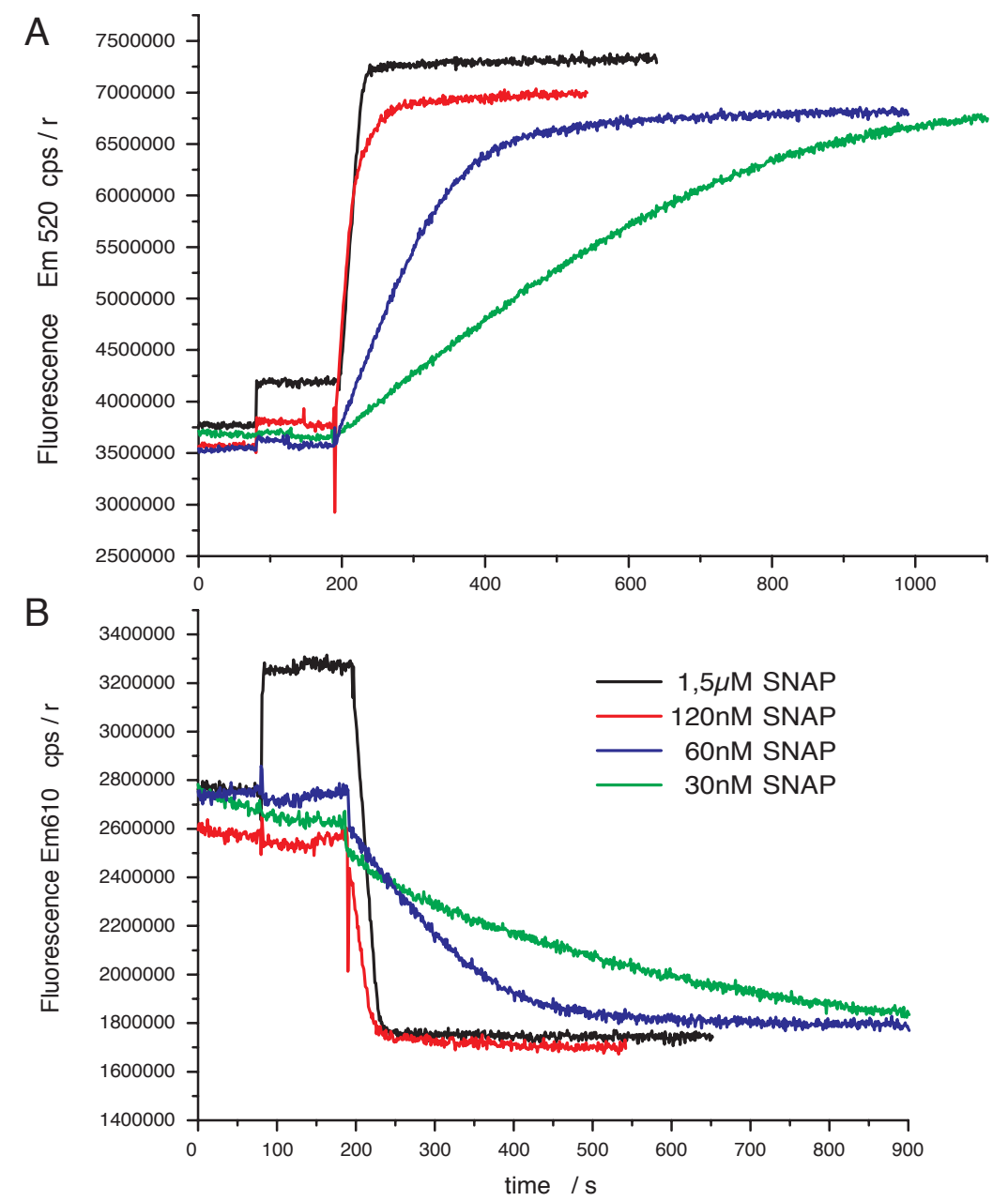

Figure 4.20: $\quad \alpha$ SNAP dependence of SNARE-complex disassembly on liposomes using FRET spectroscopy. Disassembly kinetics of $\sim 35 \mathrm{nM}$ FRET-SNARE complex $\left(\mathrm{SbTMD}^{28 T R} / \mathrm{SNAP} 25^{130 O G} / \mathrm{H} 3\right)$ with decreasing amounts of $\alpha$ SNAP in presence of $6 \mathrm{nM}$ NSF and excess of ATP (2mM) were recorded. Donor (A) and acceptor (B) fluorescence are shown. The reaction was started at $\mathrm{t}=180 \mathrm{~s}$ by addition of $\mathrm{MgCl}_{2}$. Note that $\alpha \mathrm{SNAP}$ binding leads to an increase of acceptor signal in a concentration dependent manner prior to the start of the reaction. Note that between 60 and $120 \mathrm{nM}$ of $\alpha$ SNAP suffice for optimal disassembly. 


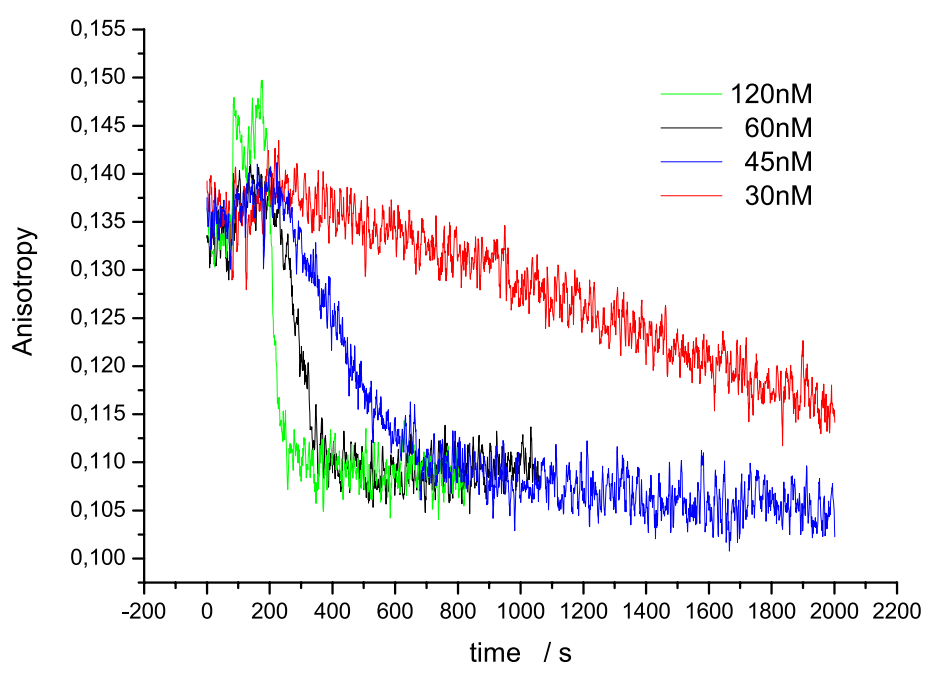

Figure 4.21: $\quad \alpha$ SNAP dependence of disassembly on liposomes using fluorescence anisotropy. Disassembly kinetics of $\sim 35 \mathrm{nM}$ SNARE complex $\left(\mathrm{SbTMD}^{28 T R} / \mathrm{SNAP} 25 / \mathrm{H} 3\right)$ using decreasing amounts of $\alpha$ SNAP in presence of $6 \mathrm{nM}$ NSF and excess of ATP $(2 \mathrm{mM})$ were recorded. The reaction was started at $\mathrm{t}=190 \mathrm{~s}$ by addition of $\mathrm{MgCl}_{2}$. Differences in fluorescence anisotropy prior to the start of the reaction result from different amounts of $\alpha$ SNAP bound to the complex (see figure 4.16). Note that again $120 \mathrm{nM}$ of $\alpha$ SNAP promote optimal disassembly.

To now determine the limiting amount for $\alpha$ SNAP under these improved conditions, decreasing amounts of $\alpha$ SNAP were used for disassembly until a concentration not capable of disassembling liposomal SNARE complexes was reached. This is illustrated in figure 4.20 using the FRET assay and in figure 4.21 using fluorescence anisotropy.

Together, these data show that $\alpha \mathrm{SNAP}$ is capable of efficiently disassembling SNARE complexes at a stoichiometric ratio of three to one or even less, even at such low concentrations. The affinity was therefore significantly increased by the incorporation of SNARE-complexes into liposomes via the Synaptobrevin transmembrane domain.

Figure 4.22 shows the comparison of disassembly kinetics in solution and on liposomes using limiting amounts of $\alpha \mathrm{SNAP}$ for the respective condition. Here one can see that $45 \mathrm{nM}$ of $\alpha \mathrm{SNAP}$ on liposomes are comparably efficient as $1,1 \mu \mathrm{M}$ in solution, leading to the conclusion that $\alpha$ SNAP is at least 20-fold more efficient on liposomes than in solution. 


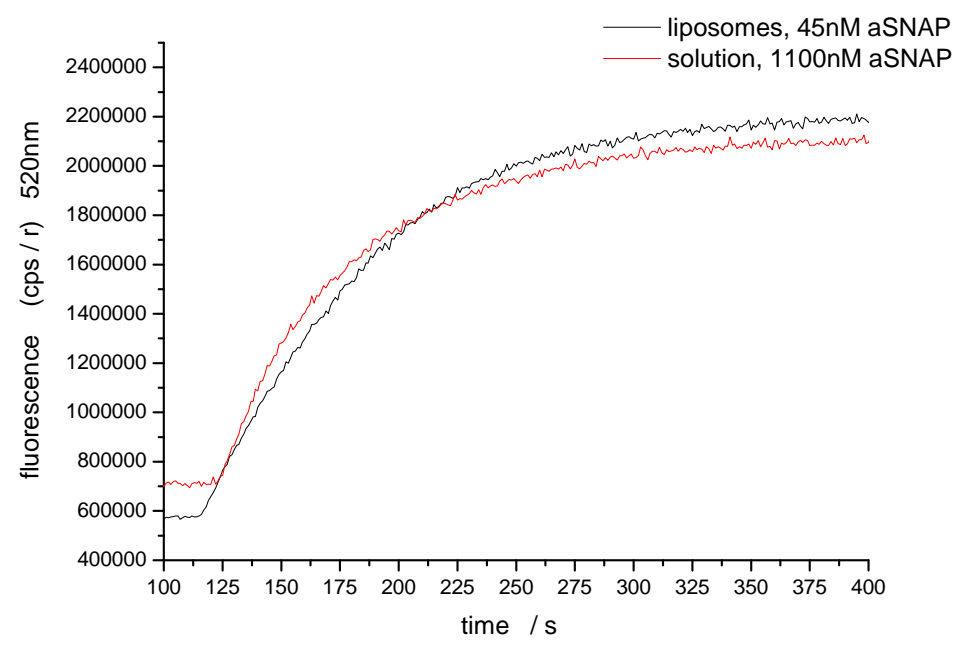

Figure 4.22: $\alpha$ SNAP is roughly 20 times more effective on liposomes than in solution. Disassembly kinetics of $\sim 70 \mathrm{nM}$ SNARE complex (H3/SNAP2 $5^{130 O G} / \mathrm{Sb}^{28 T R}$ ) either incorporated into liposomes via the Sb transmembrane domain or in solution were recorded. The reaction included $5 \mathrm{nM}$ NSF, $\alpha$ SNAP as indicated and excess of ATP $(2 \mathrm{mM})$. Disassembly was triggered after 120 s by addition of $\mathrm{MgCl}_{2}$. 
A

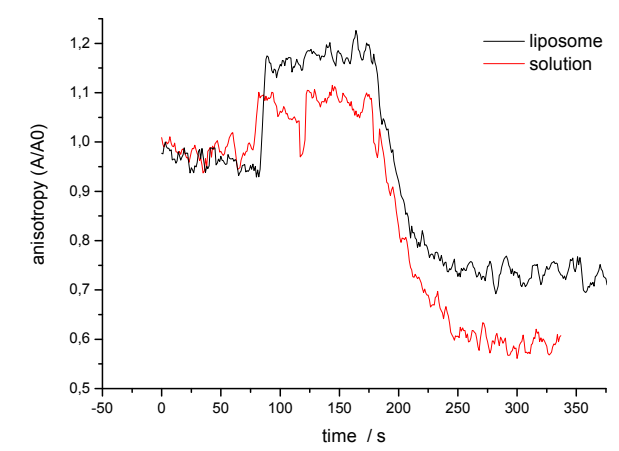

B

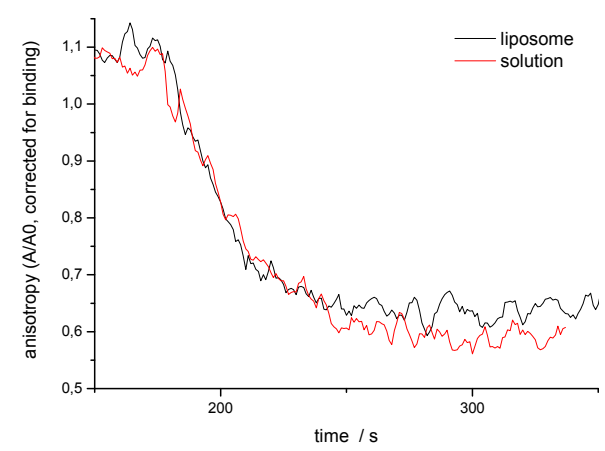

Figure 4.23: NSF is equally potent on liposomes as in solution. Liposomal and soluble complexes (H3TMD, SNAP2 $5^{130 O G} / \mathrm{Sb}$ ) were disassembled in presence of $1,25 \mu \mathrm{M} \alpha \mathrm{SNAP}$, $6 \mathrm{nM} \mathrm{NSF}$ and $2 \mathrm{mM}$ ATP by addition of $\mathrm{MgCl}_{2}$ at $\mathrm{t}=150 \mathrm{~s}$. (A) Traces represent the fluorescence anisotropy measured. (B) The solution signal has been shifted upwards to directly compare the kinetics of disassembly.

\subsubsection{NSF Performance is Unchanged on Liposomes}

To check whether NSF behaviour is changed on liposomes as well, disassembly at excess amounts of $\alpha$ SNAP $(1,5 \mu \mathrm{M})$ was compared between liposomes and solution at identical NSF-concentrations. Figure 4.23 shows that NSF disassembles at comparable rates, regardless of whether the SNARE complex is incorporated into liposomes or not, as long as $\alpha \mathrm{SNAP}$ is not limiting.

Furthermore, the same amount of anti-N-domain antibody (141.2) is needed to block NSF function on liposomes as in solution. This is illustrated in figure 4.24, where the anisotropy of liposomal SNARE complexes is recorded in presence or absence of 2,5 $\mathrm{l}$ of the ProteinA-eluted antibody 141.2. When NSF and the antibody have time to interact prior to $\alpha$ SNAP addition, disassembly is completely blocked. The block is nevertheless relieved upon addition of a further $3 \mathrm{nM}$ of NSF, identical to what was observed in solution in section 4.7. 


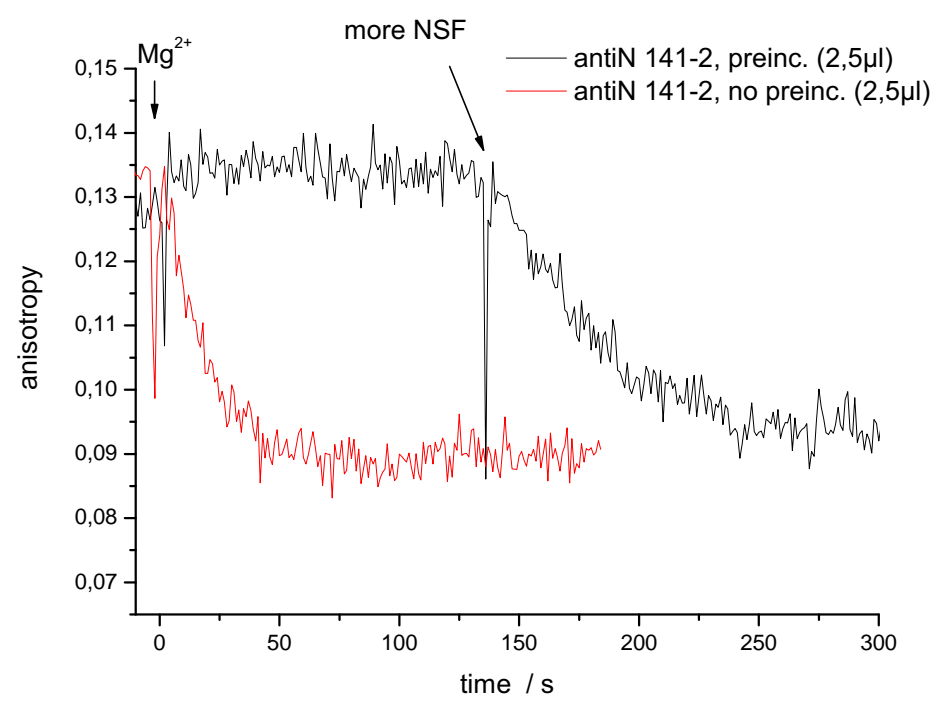

Figure 4.24: As in solution, 2,5 $\mu \mathrm{l}$ of the anti-N-domain antibody block 3nM of NSF. Black trace: Approximately 35nM of SNARE complex (H3/SNAP25/SbTMD ${ }^{28 T R}$ were mixed with $3 \mathrm{nM}$ of NSF and $2,5 \mu \mathrm{M}$ of clone 141.2 . After $50 \mathrm{~s}, 3 \mu \mathrm{M}$ of $\alpha$ SNAP were added and anisotropy recorded. The disassembly reaction was subsequently triggered by addition of $\mathrm{MgCl}_{2}$ at $\mathrm{t}=0$. Red trace: Everything as above except that $\alpha \mathrm{SNAP}$ and the antibody were added together, prior to NSF, to take away the antibodies advantage of being pre-incubated with NSF.

\section{Monitoring NSF-binding using stoichiometric amounts}
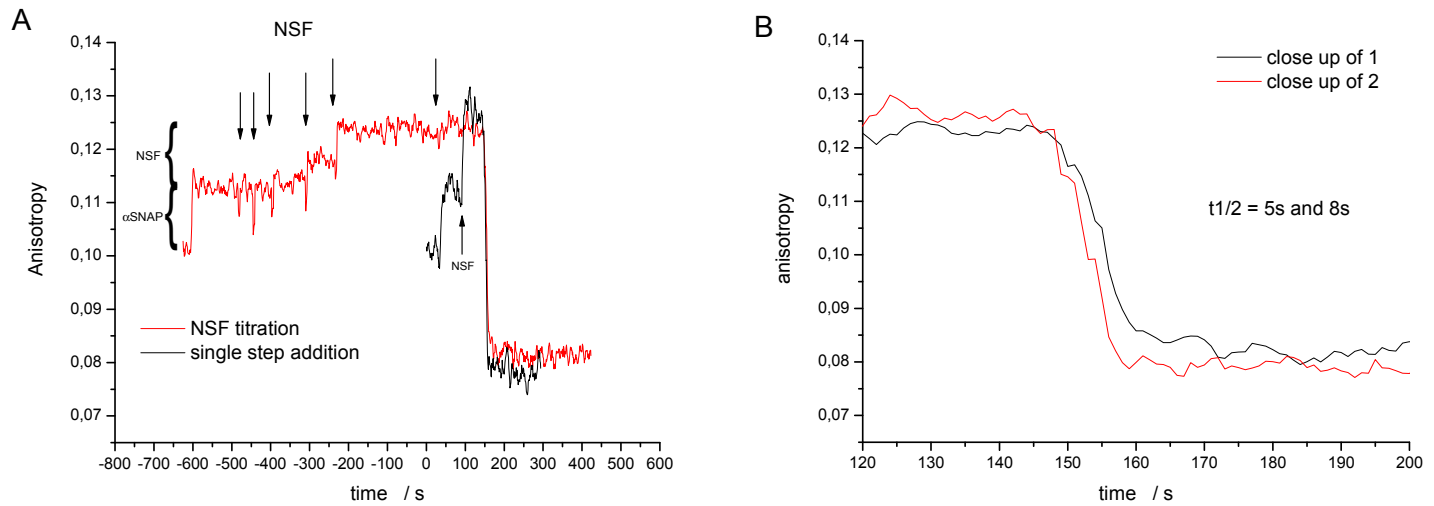

Figure 4.25: NSF-binding and speed of the reaction using stoichiometric amounts of NSF. (A) Red graph: $150 \mathrm{nM} \alpha$ SNAP were added to $\sim 35 \mathrm{nM}$ of liposomal SNARE complex $\left(\mathrm{SbTMD}^{28 T R} / \mathrm{H} 3 / \mathrm{SNAP} 25\right)$ at $\mathrm{t}=-600$ s. Subsequently NSF was added in a stepwise manner as indicated by black arrows. Finally, the reaction was started at 150 s by adding $\mathrm{MgCl}_{2}$. Black graph: Everything was performed as for the red graph, except that $\alpha \mathrm{SNAP}$ was added at $20 \mathrm{~s}$ and $15 \mathrm{nM}$ of NSF were added in a single step at $80 \mathrm{~s}$. (B) Close-up of the disassembly time interval between 120 and 200s shown on the left to illustrate the high speed of the reaction. 
To see, whether the binding of NSF to the SNARE-complex can be noted in the spectrum as well if stoichiometric instead of enzymatic amounts of NSF are added, increasing amounts of NSF were titrated to a complex already saturated with $\alpha$ SNAP $(150 \mathrm{nM})$. This is illustrated in figure $4.25(\mathrm{~A})$, where each addition of NSF is indicated by a black arrow. An increase of anisotropy is observable using such high amounts of NSF, but it is not large enough to assess stoichiometry. As can be seen on panel (B), at higher NSF concentrations the rate of disassembly soon becomes too fast to be resolved with this experimental set-up. The apparent half-times of disassembly here are 5 and 8 seconds, which probably is an underestimate since this time period is in the range of the time required for efficient mixing of the reaction solution.

A time resolution high enough to obtain a spectrum of one disassembly cycle using stoichiometric amounts of the SNAREs, SNAPs and NSF might be achieved using a stopped-flow apparatus. Some preliminary experiments have already been performed using such a set-up, pointing to a speed of less than four seconds per cycle at room temperature (data not shown). Since NSF has been observed to disassemble significantly faster at $37^{\circ} \mathrm{C}$, an even faster rate of disassembly can be expected at that temperature. Here, it should nevertheless be stated again that these in vitro experiments cannot provide absolute numbers with respect to physiological processes but only serve as a platform to pursue comparative investigations. 


\subsection{Membrane Anchorage of SNAREs Potenti- ates the Power of $\alpha$ SNAP}

Looking at the experiments explained in the last section it became evident that much less $\alpha$ SNAP is needed for efficient disassembly using complexes incorporated into liposomes via the transmembrane domain (TMD) of Synaptobrevin. Nevertheless it is as yet still unclear, whether the liposomes or the TMD of Synaptobrevin are the factor causing the improved function.

\subsubsection{The Transmembrane Domain of Synaptobrevin is not Essential for $\alpha$ SNAP Potentiation}

To test whether the Synaptobrevin transmembrane domain increases $\alpha$ SNAP function, SNARE complexes lacking the Synaptobrevin transmembrane domain were incorporated into liposomes via the Syntaxin transmembrane domain. As judged from my previous findings, 300nM $\alpha$ SNAP are not capable of mediating sufficient disassembly in solution but are sufficient for efficient disassembly on liposomal complexes incorporated via the Sb-transmembrane domain.

To now determine, whether liposomal complexes incorporated via the Syntaxinand hence missing the Sb-transmembrane domain mediate disassembly at 300nM $\alpha$ SNAP, populations of complexes with a TMD of either H3 or full length Syntaxin were disassembled by two different concentrations of $\alpha \mathrm{SNAP}(300 \mathrm{nM}$ and $1,5 \mu \mathrm{M}$, respectively).

Notably, as shown in figure $4.26,300 \mathrm{nM} \alpha$ SNAP were not limiting during complex disassembly. This can be concluded from the fact that the speed of disassembly at the two concentrations used was identical. Furthermore, the binding of both amounts of $\alpha$ SNAP results in a comparable increase of fluorescence anisotropy, once more indicating that the system is saturated at $300 \mathrm{nM} \alpha$ SNAP. Moreover, the disassembly kinetics were still identical when the NSF concentration was raised from $1,5 \mathrm{nM}$ to $6 \mathrm{nM}$, indicating that even at higher NSF concentrations $300 \mathrm{nM}$ of $\alpha \mathrm{SNAP}$ are not limiting as opposed to what would be expected in solution.

Indeed, performing the same experiment in solution (figure 4.27) confirmed that the lower $\alpha$ SNAP concentration here only allows for slow disassembly of the soluble complexes. The slow disassembly may not even be improved by raising the NSF concentration. Disassembly is hence improved on liposomes at low $\alpha$ SNAP levels even in the absence of the Synaptobrevin TMD, showing that it is not the TMD of Synaptobrevin which leads to the observed increase of $\alpha$ SNAP affinity on liposomes. 

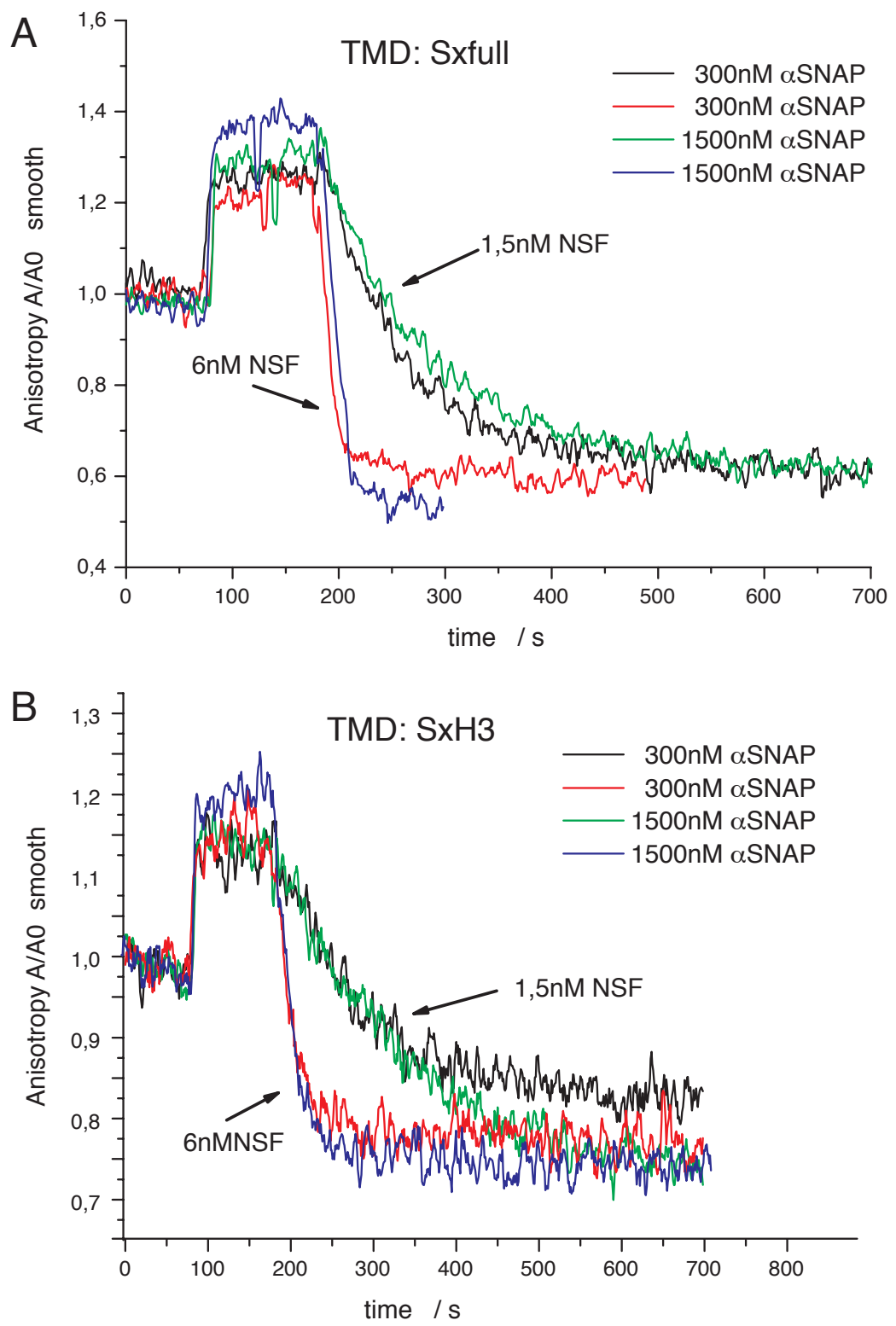

Figure 4.26: $300 \mathrm{nM} \quad \alpha \mathrm{SNAP}$ are not limiting even in the absence of the Synaptobrevin transmembrane domain. (A) Full-length Syntaxin complexes (SxTMD/SNAP25 $\left.5^{130 O G} / \mathrm{Sb}\right)$ were pre-assembled and incorporated into liposomes via the Syntaxin transmembrane domain. (B) Complexes containing Syntaxin without Habc domain (H3TMD/SNAP25 $5^{130 O G} / \mathrm{Sb}$ ) were pre-assembled and incorporated into liposomes via the Syntaxin transmembrane domain. The $35 \mathrm{nM}$ complex per reaction were dissociated using $1,5 \mathrm{nM}$ or $6 \mathrm{nM}$ of NSF and $300 \mathrm{nM}$ or $1,5 \mu \mathrm{M}$ of $\alpha \mathrm{SNAP}$ and started at $\mathrm{t}=190 \mathrm{~s}$ by addition of $\mathrm{MgCl}_{2}$. Notably, the binding of both amounts of $\alpha$ SNAP results in a comparable increase of fluorescence anisotropy $(\mathrm{t}=50 \mathrm{~s})$. 


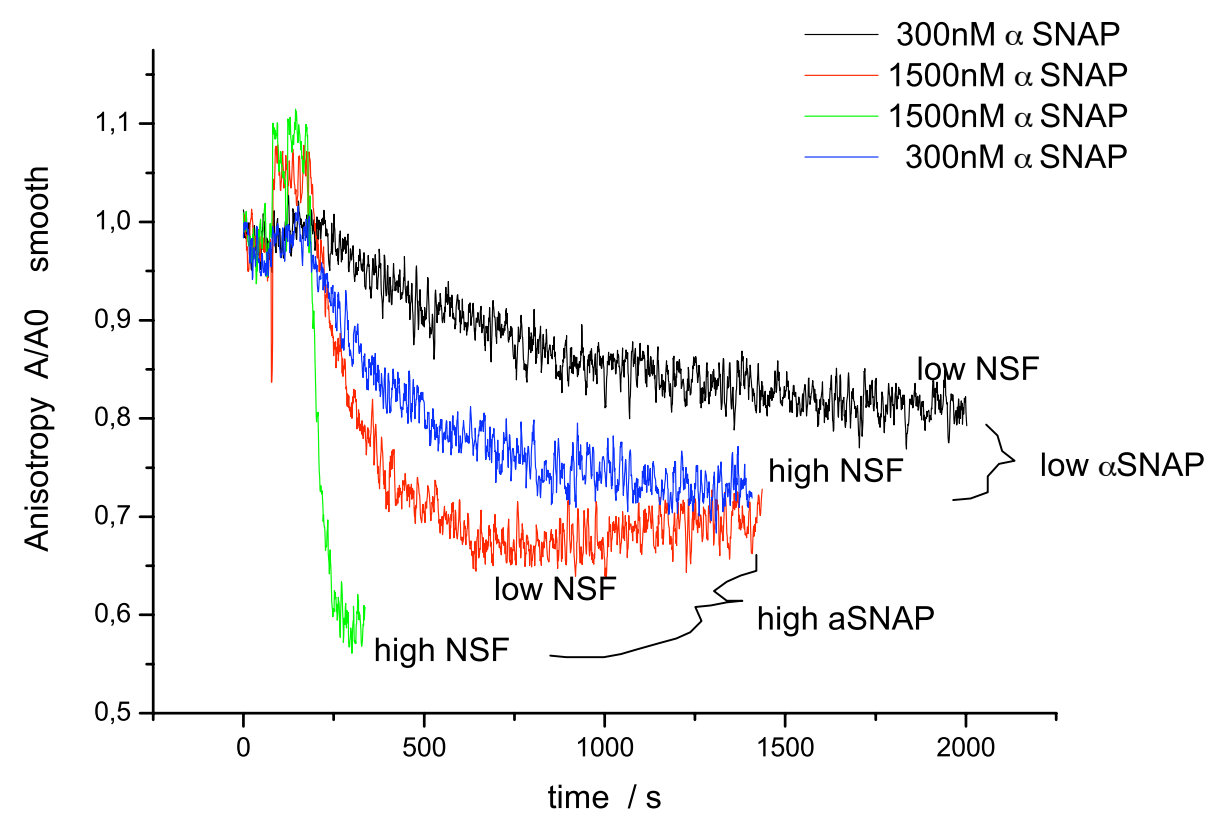

Figure 4.27: $300 \mathrm{nM}$ of $\alpha \mathrm{SNAP}$ are limiting using the same amount of soluble complexes. The procedure and conditions were identical to figure 4.26, except that soluble complex (H3/Syb/SNAP25 ${ }^{130 O G}$ ) not incorporated into liposomes was disassembled. The $35 \mathrm{nM}$ complex per reaction were dissociated using $1,5 \mathrm{nM}$ or $6 \mathrm{nM}$ of NSF and $300 \mathrm{nM}$ or $1,5 \mu \mathrm{M}$ of $\alpha$ SNAP and started at $\mathrm{t}=190 \mathrm{~s}$ by addition of $\mathrm{MgCl}_{2}$. Note that the anisotropy shift caused by $\alpha$ SNAP binding (at $\mathrm{t}=30 \mathrm{~s}$ ) is far more pronounced for the binding of $1,5 \mu \mathrm{M}$ than for that of $300 \mathrm{nM} \alpha$ SNAP. 


\subsubsection{The Potentiation of $\alpha$ SNAP Efficacy is Independent of the Lipid Composition}

Now knowing that liposomes increase $\alpha$ SNAP efficacy, it remained to be elucidated whether this effect is caused by a specific lipid component. The liposomes up to this stage were constituted of phosphatidylcholine (PC), phosphatidylethanolamine (PE), cholesterol (Chol), phosphatidylserine (PS), phosphatidylinositol (PI) in order to closely resemble the lipid composition of the brain. To repeat the experiments with a simplified lipid composition, the liposomes used in the following experiment were constituted of PC and PE only. Even this simple lipid composition did not alter the efficiency of $\alpha \mathrm{SNAP}$ (figure 4.28). As shown here, $60 \mathrm{nM}$ of $\alpha \mathrm{SNAP}$ were sufficient to drive the reaction, which is comparable to the findings for the more complex liposomal preparations used in the previous experiments. It turned out that even pure phosphatidylcholine liposomes (data not shown) suffice to support the increased potency of $\alpha \mathrm{SNAP}$. The effect is hence caused not by any special lipid but rather independent of the composition of lipids.

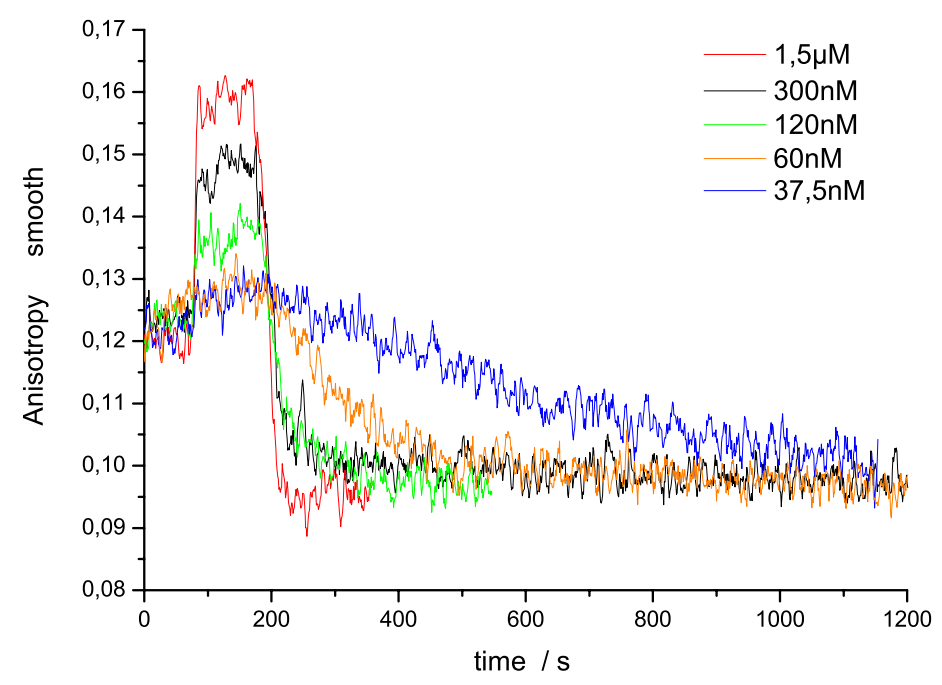

Figure 4.28: Liposomes consisting of PC and PE only are sufficient to bring about the observed higher efficacy. The experiment was performed as in figure 4.21 except that cholesterol, PI and PS were omitted from the lipid mixes used to generate the liposomes. The reactions were started at $\mathrm{t}=190 \mathrm{~s}$. 


\subsubsection{Disassembly on Partially Solubilized Liposome Popu- lations (Mixed Micelles)}

To ensure that it is not the mere presence of lipids in the environment (which e.g. might stabilize fragile regions of $\alpha \mathrm{SNAP}$ and thereby promote a positive effect), but indeed the anchorage of the complex to the membrane, the following strategy was employed: First, it should be investigated how much of a respective detergent is needed to solubilize only a fraction of the liposomes, leaving the rest intact. Afterwards, disassembly was to be tested at different ratios of micelles to liposomes caused by different amounts of detergent. Under such conditions, the amount of disassembly in presence of low $\alpha \mathrm{SNAP}$ should correlate with the fraction of complexes still incorporated into liposomes, whereas dissociation of the remaining complexes should only proceed when the $\alpha$ SNAP concentration would be increased to levels sufficient for disassembly in solution.

\section{Disassembly in Presence of Dodecylmaltoside}

To determine the detergent concentration required for partial solubilization, detergent was titrated into liposomes that contained 1,5\% NBD (Nitrobenzoxadiazole)and 1,5\% Rhodamine-labelled lipids, respectively, two dyes which can function as a FRET pair. With increasing detergent concentrations, FRET efficiency between the lipids will at first be slightly perturbed by insertion of detergent molecules into liposomes and at some point dramatically decrease due to solubilization of the liposomes, which obviously leads to an increased distance between the lipids. Several detergents were tried, the biggest difficulty being the circumstance that most detergents, when used at concentrations that solubilize liposomes, harm the efficiency of the disassembly reaction as such. These are consequently not suited for experiments comparing disassembly at different detergent concentrations. Dodecylmaltoside (DOM) however, a detergent which due to its low critical micelle concentration (cmc) succeeds to solubilize liposomes already at low concentrations, turned out not to have a negative impact on disassembly activity in the respective concentration range.

In figure 4.29 the increase of donor fluorescence during DOM-titration into labelled liposomes is shown and the fraction of solubilized liposomes estimated. Below, disassembly is performed on mixed micelles, representing a mixture of liposomeattached and detergent-solubilized FRET-labelled SNARE complexes at the respective detergent concentrations. For the first step of disassembly, enough $\alpha$ SNAP (i.e. $120 \mathrm{nM}$ ) to disassembly liposomal but not soluble complexes according to what we learned in section 4.3.3 was applied. After stabilization of the signal subsequent to this first part of disassembly, a high concentration of $\alpha$ SNAP suited to disassemble complexes in solution was added and the remainder of disassembly proceeded. Figure 4.30 lists the correlation between the fraction of liposomes expected to exist and the amount of disassembly proceeding at the low $\alpha$ SNAP concentration under the respective concentration of detergent. 

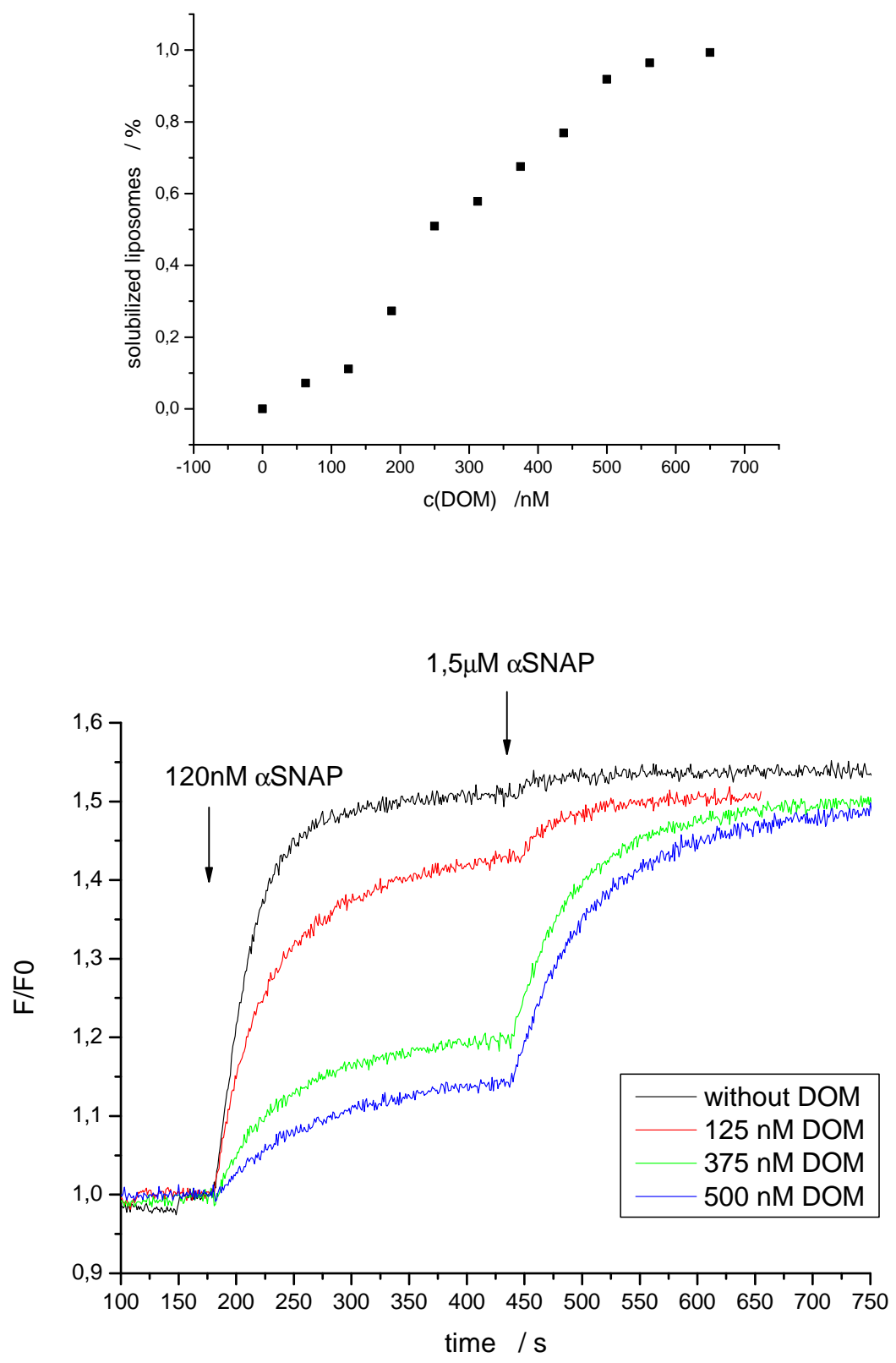

Figure 4.29: The percentage of disassembly requiring low $\alpha$ SNAP correlates with the fraction of liposomal complexes in mixed micelle solutions. (A) The donor fluorescence during titration of DOM into Nbd/Rho-labelled liposomes was recorded, used to estimate the fraction of solubilized liposomes (\% solubilized liposomes $\left.=\frac{F-F_{\text {start }}}{F_{\text {end }}-F_{\text {start }}}, F_{\text {start }}\right)$ and plotted against the concentration of DOM. (B) Disassembly is performed on mixed micelles at DOM concentrations of 125,375 and $500 \mathrm{nM}$, respectively. After $160 \mathrm{~s}, 120 \mathrm{nM} \alpha \mathrm{SNAP}$ were added to allow for disassembly of liposomal SNAREs. At $\mathrm{t}=440 \mathrm{~s}$, a further $1,5 \mu \mathrm{M}$ of $\alpha$ SNAP were added to also allow for disassembly of the soluble SNARE complexes. 


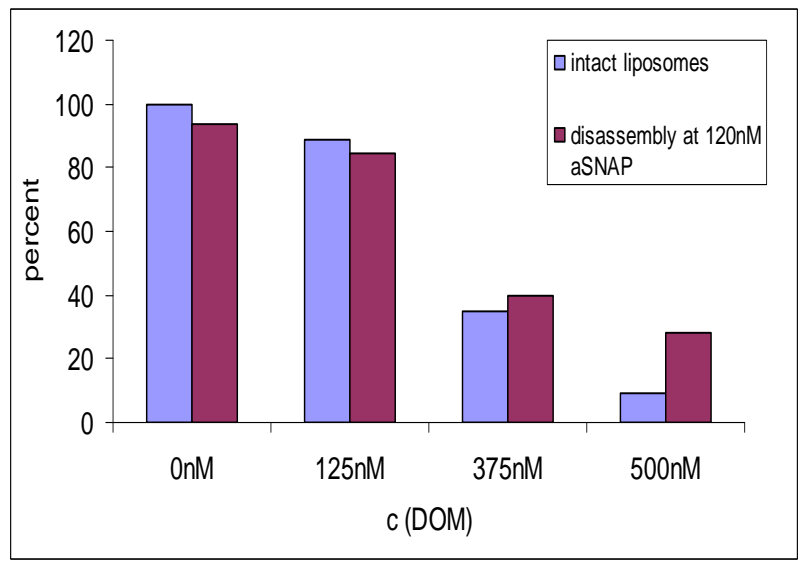

Figure 4.30: The percentage of disassembly requiring low $\alpha$ SNAP correlates with the fraction of liposomal complexes in mixed micelle solutions. 


\subsubsection{An $\alpha$ SNAP Mutant Lacking the Putative Membrane Interaction}

The data collected so far made it tempting to speculate that a direct interaction between $\alpha$ SNAP and the membrane lipids is responsible for the increased efficacy on liposomes. If this were true, the lipid binding property might possible be mapped to a certain region of $\alpha$ SNAP. No crystal structure has been solved for $\alpha$ SNAP so far, but the structure of the $\alpha$ SNAP isoform $\gamma$ SNAP as well as the yeast $\alpha$ SNAP homolog Sec17 have been solved [30]. Based on the Sec17-structure and $\alpha$ SNAP/SNARE-complex interaction studies using various point-mutated $\alpha$ SNAPs, a model of $\alpha$ SNAP bound to the SNARE complex [67] has been proposed by Marz et al.. Looking closely at the model one can observe that the very N-terminal 32 residues form an arm-like structure pointing away from the complex, the most farout region of which includes a strand of mostly hydrophobic amino acids. If this region were the interaction site of $\alpha \mathrm{SNAP}$ with the membrane, its deletion should abolish membrane binding and hence the higher $\alpha$ SNAP efficiency on membranes.

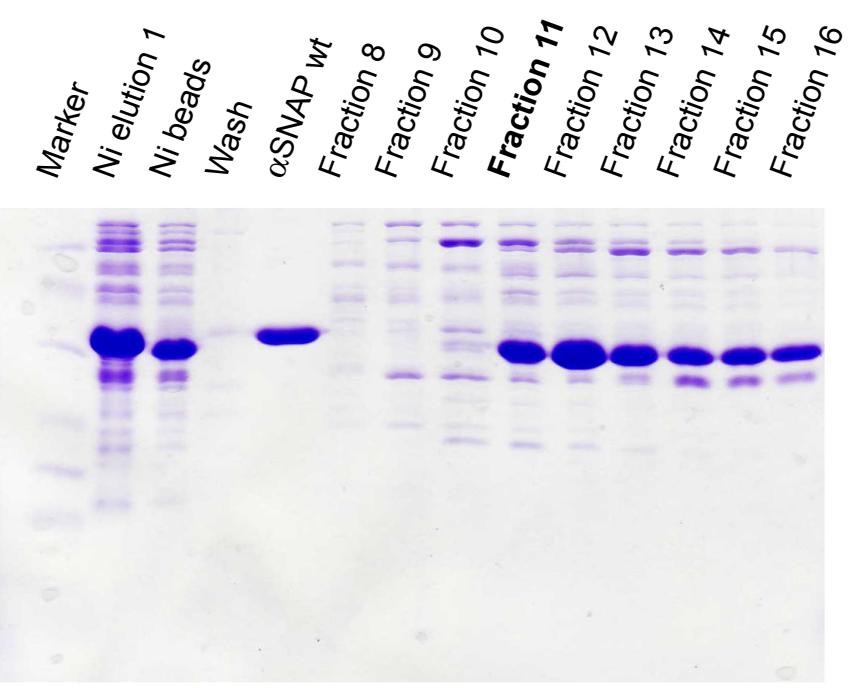

Figure 4.31: Purification of the $\alpha$ SNAP mutant ' $\alpha$ delSNAP'. A sample after elution from the Ni column, $\alpha$ SNAP wildtype for size comparison as well as the elution fractions after anion-exchange chromatography are shown. Fraction 11 was used for all of the functional experiments.

A deletion mutant comprising aa 33-295 of $\alpha$ SNAP was hence cloned into the pET28a vector and subsequently expressed and purified. It will be referred to as ' $\alpha$ delSNAP' from now on. The purification of the His-tagged protein was carried out by Wolfgang Berning-Koch via Ni/NTA- followed by anion-exchange chromatography. It worked according to the standard protocol used for $\alpha$ SNAP 4.31 .

First, I tested whether $\alpha$ delSNAP is able to disassemble SNARE complexes using the previously described FRET assay. Indeed, $\alpha$ delSNAP is able to disassemble - 


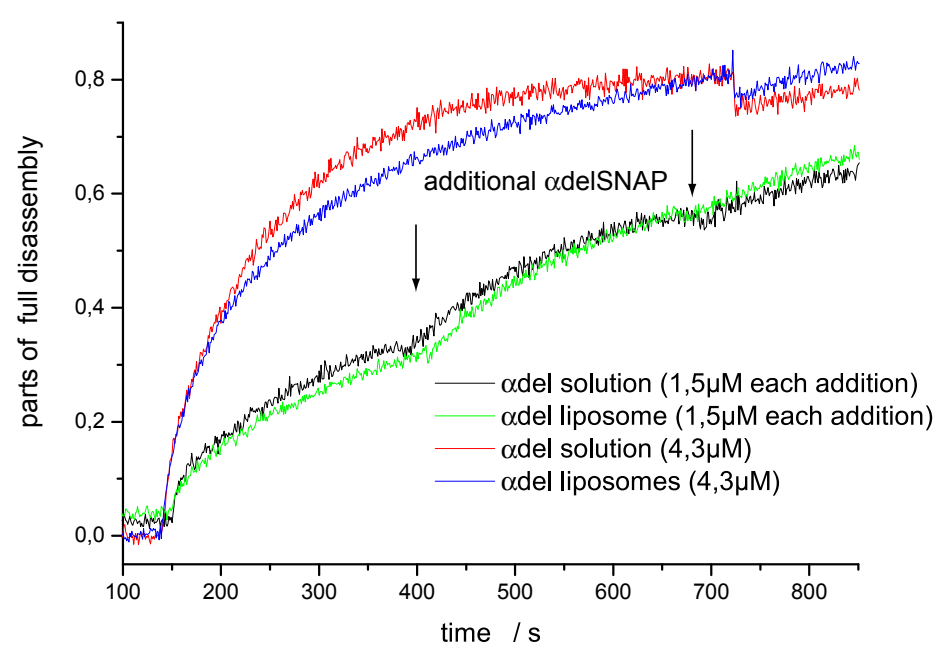

Figure 4.32: $\alpha$ delSNAP efficiency is comparable on liposomes and in solution. Disassembly is shown $\sim 70 \mathrm{nM}$ of FRET complexes (H3/SNAP2 $5^{130 O G} / \mathrm{Sb}^{28 T R}$ ) either in solution or incorporated into liposomes via the TMD of Sb. At the start of the reaction the samples contained the amount of $\alpha$ del indicated. The reactions were triggered after $140 \mathrm{~s} .1,5 \mu \mathrm{M}$ of $\alpha$ delSNAP were added twice more to the reactions represented in black and green, at 400 s and 680s.

albeit at lower efficiency than $\alpha$ SNAP. At an $\alpha$ delSNAP concentration of $1,5 \mu \mathrm{M}$, disassembly was slow and only a third of the substrate was disassembled. In order to improve the rate of disassembly, more $\alpha$ delSNAP had to be added. $4,4 \mu \mathrm{M}$ succeeded to efficiently disassemble most of the complex (figure 4.32).

Next, to address the question of whether the membrane boost observed for wt$\alpha \mathrm{SNAP}$ in the previous sections is indeed abolished in the mutant, the kinetics of $\alpha$ delSNAP mediated disassembly in solution and on liposomes were compared. As illustrated in figure 4.32, for all concentrations of $\alpha$ delSNAP tested the kinetics in solution are identical to the kinetics on liposomes. This result indicates that the membrane mediated $\alpha$ SNAP-potentiation was indeed abolished by deletion of its N-terminal region.

To strengthen the finding that $\alpha$ delSNAP does not differentiate between liposomal and soluble complexes with respect to disassembly efficiency, the anisotropy read-out was employed next and $\alpha$ delSNAP directly compared to wildtype $\alpha$ SNAP. Since in the FRET-experiment it already became obvious that $\alpha$ delSNAP is less effective than wildtype $\alpha \mathrm{SNAP}$ in general, the extent of this difference was determined at first. To do so, various amounts of $\alpha$ delSNAP and $\alpha$ SNAP were compared with respect to their ability to disassemble SNARE complexes in solution. As shown in figure $4.33,3,6 \mu \mathrm{M}$ of $\alpha$ delSNAP disassemble complexes with the same kinetics as $600 \mathrm{nM} \alpha$ SNAP. Likewise, $1,8 \mu \mathrm{M}$ of $\alpha$ delSNAP are as efficient as $300 \mathrm{nM} \alpha \mathrm{SNAP}$. It can hence be concluded that $\alpha$ delSNAP is six times less efficient than $\alpha$ SNAP in solution (figure 4.33). 


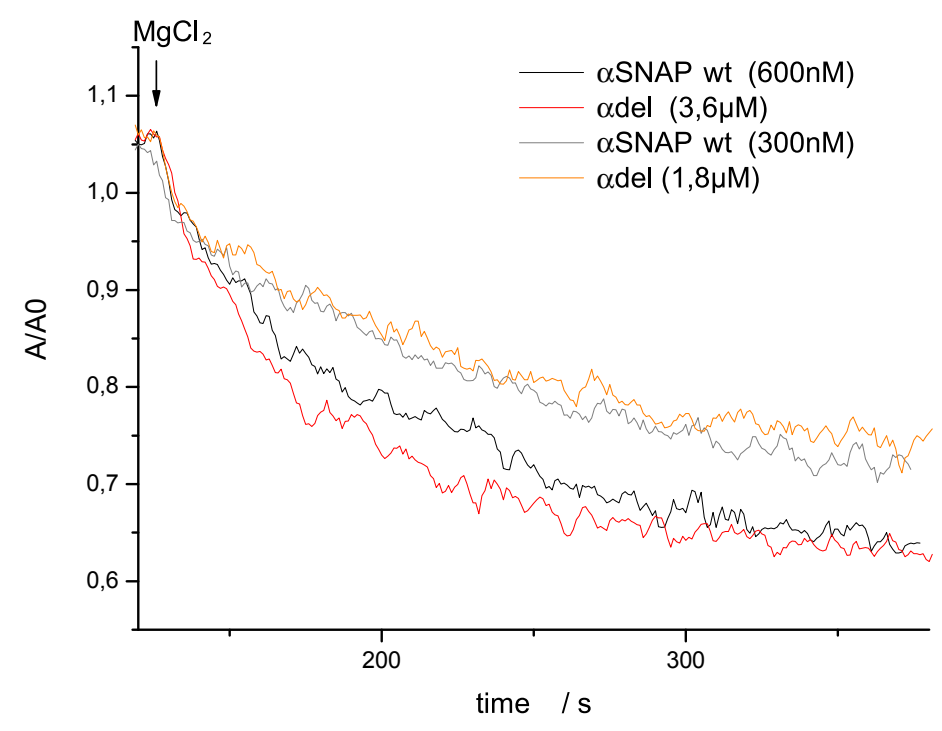

Figure 4.33: $\alpha$ delSNAP is less efficient than $\alpha$ SNAP in solution. The fluorescence anisotropy changes upon disassembly of purified SNARE complexes (H3/SNAP25/Sb $\mathrm{Sb}^{28 T}$ ) with either $\alpha$ SNAP or $\alpha$ delSNAP as the NSF/SNARE-adaptor protein are shown. The signal at a given time is divided by the signal prior to the adaptor-addition $\left(\mathrm{A} / \mathrm{A}_{0}\right)$. The reaction was triggered after 120 s as indicated.

Subsequently, the efficiencies of $\alpha$ delSNAP and $\alpha$ SNAP with respect to disassembly were compared on liposomes. First, I employed the concentrations of $\alpha$ delSNAP and $\alpha$ SNAP which led to comparable kinetics in solution. On liposomes, 600nM of $\alpha$ SNAP disassembled the SNARE complexes significantly faster than $3,6 \mu \mathrm{M}$ of $\alpha$ delSNAP (figure 4.34). At lower $\alpha$ SNAP concentrations, which are more appropriate for $\alpha$ SNAP disassembly on liposomes, the differences between $\alpha$ delSNAPand $\alpha$ SNAP-mediated kinetics at a ratio of $6: 1$ are even more pronounced: $280 \mathrm{nM}$ $\alpha$ delSNAP do not permit any disassembly at all, whereas $45 \mathrm{nM}$ of $\alpha \mathrm{SNAP}$ mediate fast and complete disassembly (figure 4.35). Even after increasing the concentration of $\alpha$ delSNAP up to $2,3 \mu \mathrm{M}$ the rate of $\alpha$ delSNAP mediated disassembly was slower than that of the wildtype reaction (data not shown). These results are in line with the outcome of the FRET assay, that $\alpha$ delSNAP does not preferentially disassemble on liposomes. 


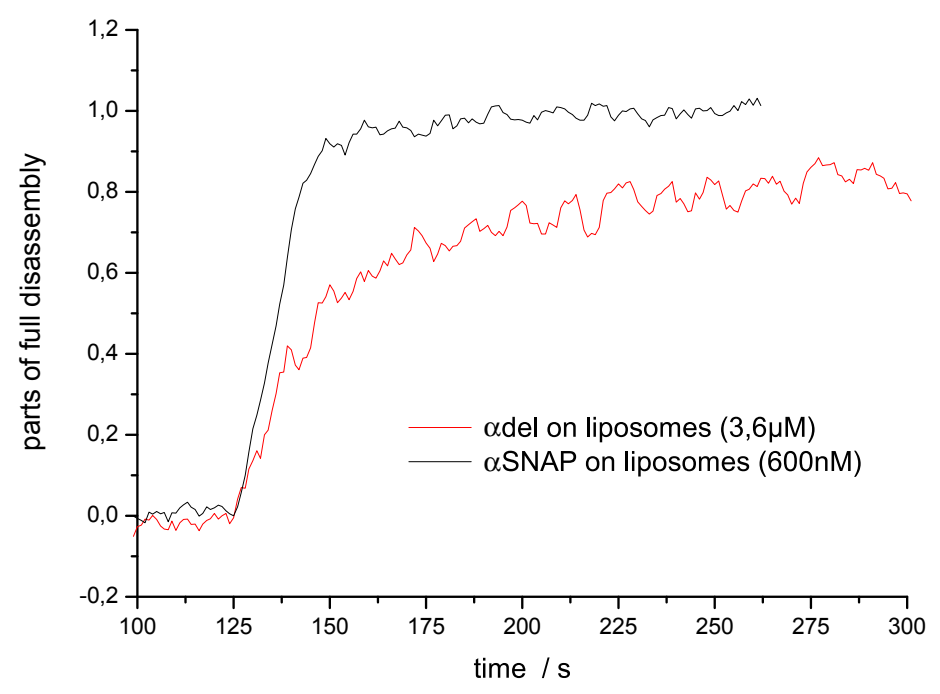

Figure 4.34: The difference between $\alpha$ delSNAP and $\alpha$ SNAP is far more pronounced on liposomes than in solution. Anisotropy changes during disassembly of $\sim 70 \mathrm{nM}$ liposomal SNARE complexes (H3/SNAP25/Sb $28 T R$ TMD) at $3,6 \mu \mathrm{M} \alpha$ del or $600 \mathrm{nM} \alpha \mathrm{SNAP}$ in the presence of $5 \mathrm{nM}$ NSF. Disassembly was triggered at $130 \mathrm{~s}$ by $\mathrm{MgCl}_{2}$. Anisotropy was normalized to parts of full disassembly to ease quantification.

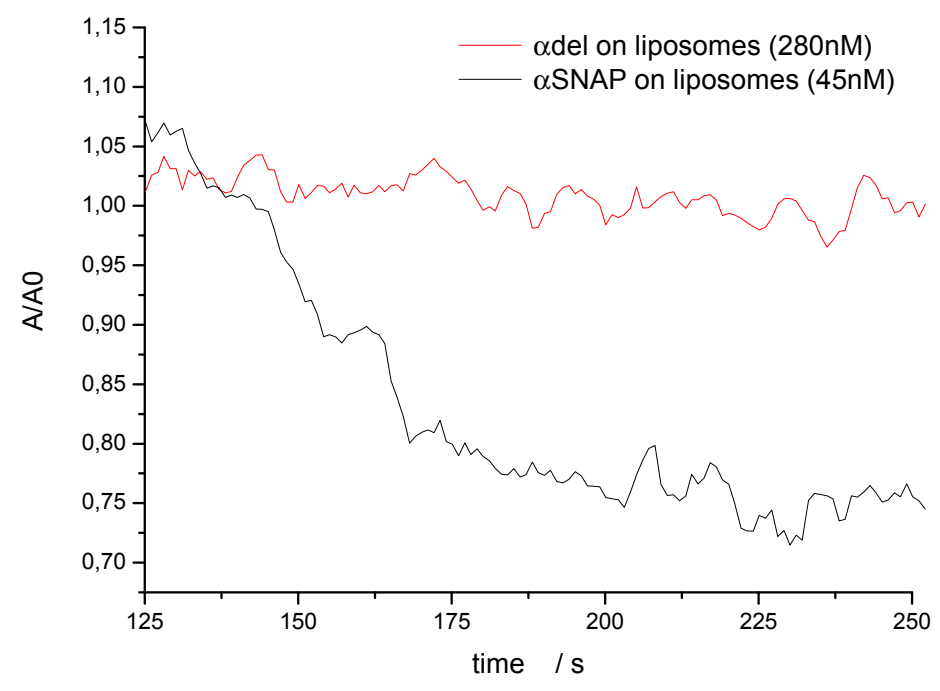

Figure 4.35: The low $\alpha$ delSNAP efficacy on liposomes is even more obvious at low SNAP concentrations. Anisotropy traces of SNARE-complex disassembly (H3/SNAP25/Sb $\left.{ }^{28 T R} \mathrm{TMD}\right)$ at $280 \mathrm{nM} \alpha$ delSNAP or $45 \mathrm{nM} \alpha \mathrm{SNAP}$ in the presence of $5 \mathrm{nM}$ NSF. Disassembly was triggered at $125 \mathrm{~s}$ by $\mathrm{MgCl}_{2}$.

Theoretically, from the findings we have so far, one can predict the factor by which wt- $\alpha$ SNAP should be more efficient than $\alpha$ delSNAP during SNARE-complex 


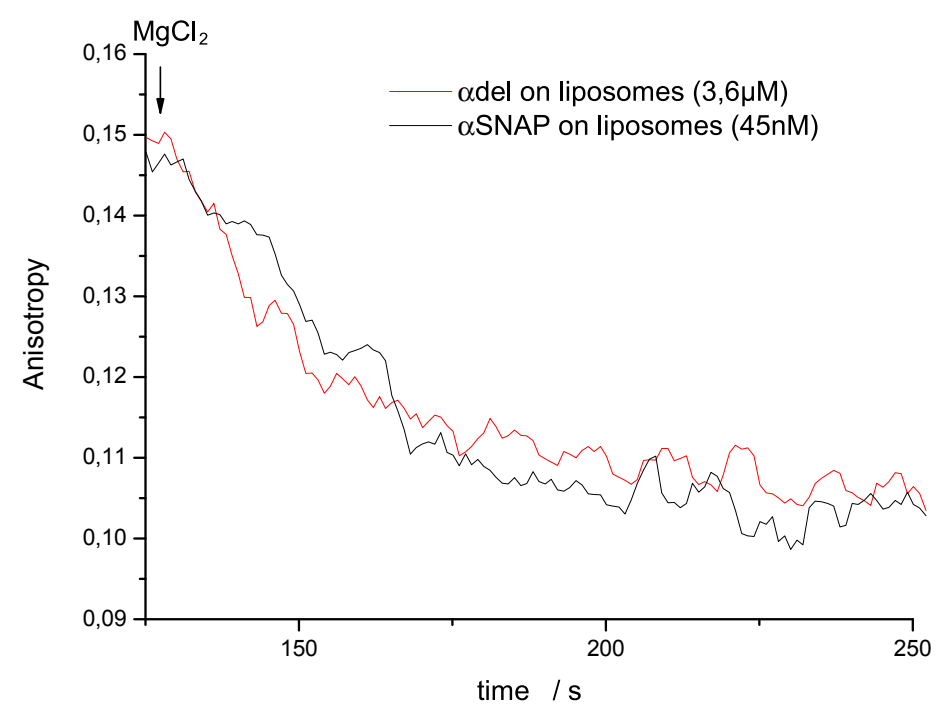

Figure 4.36: $45 \mathrm{nM} \alpha$ SNAP disassemble liposomal SNARE complexes at a comparable speed as 3,6 $\mu \mathrm{M}$ of $\alpha$ delSNAP. Anisotropy traces of liposomal SNARE-complex disassembly (H3/SNAP25/Sb $\left.{ }^{28 T R} \mathrm{TMD}\right)$ at $3,6 \mu \mathrm{M} \alpha \mathrm{del}$ or $45 \mathrm{nM} \alpha \mathrm{SNAP}$ in the presence of $5 \mathrm{nM}$ NSF. Disassembly was triggered at $125 \mathrm{~s}$ by $\mathrm{MgCl}_{2}$ as indicated.

disassembly on liposomes. We have seen in this section that $\alpha$ delSNAP is approximately six fold less efficient than $\alpha$ SNAP in solution, and in section 4.3.3 that the efficiency of wildtype $\alpha \mathrm{SNAP}$ is increased by a factor of 10-20 in the presence of membranes. According to this, an efficacy factor in the range of 60-120 (roughly six times ten to twenty) would be expected on liposomes. Indeed, figure 4.36 illustrates that $45 \mathrm{nM} \alpha \mathrm{SNAP}$ disassemble liposomal SNARE complexes at similar speed as 3,6 $\mu \mathrm{M}$ of $\alpha$ delSNAP, indicating an 80-fold higher efficiency for $\alpha$ SNAP. These findings strongly suggest that the N-terminal arm-like structure observable in the crystal structure of $\alpha \mathrm{SNAP}$ is essential for a protein/lipid-interaction, which mediates the potentiation of complex disassembly at low $\alpha \mathrm{SNAP}$ concentrations.

\subsubsection{Potentiation Through Membrane Anchorage - Gen- eral Feature of SNAPs or $\alpha$ SNAP-Specific Phenomenon?}

The adaptor $-\alpha$ SNAP versus its brain specific isoform $\beta$ SNAP

As described in the introduction, $\alpha$ SNAP is not the only adaptor protein of NSF. Two more $\alpha$ SNAP isoforms are known, called $\beta$ - and $\gamma$ SNAP, respectively. $\gamma$ SNAP is ubiquitously expressed like $\alpha$ SNAP, whereas $\beta$ SNAP is a brain specific isoform. Up to date there have been contradictory results about $\beta$ SNAP function and its ability to disassemble SNARE complexes in particular and it yet remains unclear whether $\beta$ SNAP functions as a positive or negative regulator or simply provides redundancy despite relatively low sequence homology [32, 33, 31, 34]. 
With the following experiments I sought to answer, whether $\beta$ SNAP is capable of disassembling SNARE-complexes and if so, whether its properties differ from those of $\alpha$ SNAP. For instance, it is as yet not known, whether $\alpha$ SNAP has the same affinity for all three binding sites and whether all three binding sites need to be occupied to allow for disassembly. If the SNAP binding sites are not exchangeable there might be differences of $\beta$ - and $\alpha$ SNAP affinities to any of the binding sites. If so, it is for instance feasible that $\alpha$ - and $\beta$ SNAP are more or less efficient in different concentration ranges, respectively and that mixing of both SNAP isoforms would lead to different kinetics than the sum of both isoforms alone. Furthermore, an interesting question would be, whether the membrane boost observed for $\alpha$ SNAP on liposomes is conserved between SNAP isoforms.

The properties of $\alpha$ - and $\beta$ SNAP are similar in solution To first establish, whether $\beta$ SNAP disassembles neuronal SNARE complexes in general, I purified recombinant $\beta$ SNAP and used it for disassembly experiments exploiting the fluorescence anisotropy.

As can be seen in figure 4.37, $\beta$ SNAP was able to disassemble the SNARE complex. Like previously observed for $\alpha$ SNAP, the binding of $\beta$ SNAP to the SNAREcomplex results in an increase of fluorescence anisotropy. All experiments were also performed with $\alpha$ SNAP as a direct comparison. It should be noted that $\beta$ SNAP turned out to be fragile during purification, making it impossible to estimate the true power of $\beta$ SNAP as compared to $\alpha$ SNAP. The following experiments are hence not intended to provide absolute numbers but only aim to investigate whether there are mechanistical differences between the SNAP isoforms.

As is shown in figure $4.37,3,4 \mu \mathrm{M} \beta \mathrm{SNAP}$ disassembled the complexes with the same kinetics as $600 \mathrm{nM} \alpha \mathrm{SNAP}$. The $\beta$ SNAP preparation used was hence about four times less effective than $\alpha$ SNAP at this concentration. To see, whether this difference is reproducible at much lower concentrations, a second experiment using only $600 \mathrm{nM}$ $\beta$ SNAP and $150 \mathrm{nM} \alpha \mathrm{SNAP}$ was carried out. Again, the kinetics were comparable. Further experiments were pursued using different $\beta$ - and $\alpha$ SNAP concentrations, all of which lead to comparable kinetics of $\alpha$ - and $\beta$ SNAP-containing reactions, when for times more $\beta$ SNAP preparation than $\alpha$ SNAP was used (data not shown). Furthermore, mixing of limiting amounts $\alpha$ SNAP and $\beta$ SNAP resulted in a reaction rate which would be expected for the sum of the two isoforms (data not shown). None of these solution-experiments hence pointed towards a different function of $\beta$ SNAP and $\alpha \mathrm{SNAP}$, rather suggesting that both isoforms are mechanistically exchangeable in solution. Whether the efficiencies of the isoforms differ, can not be judged from these experiments, as pointed out above. 

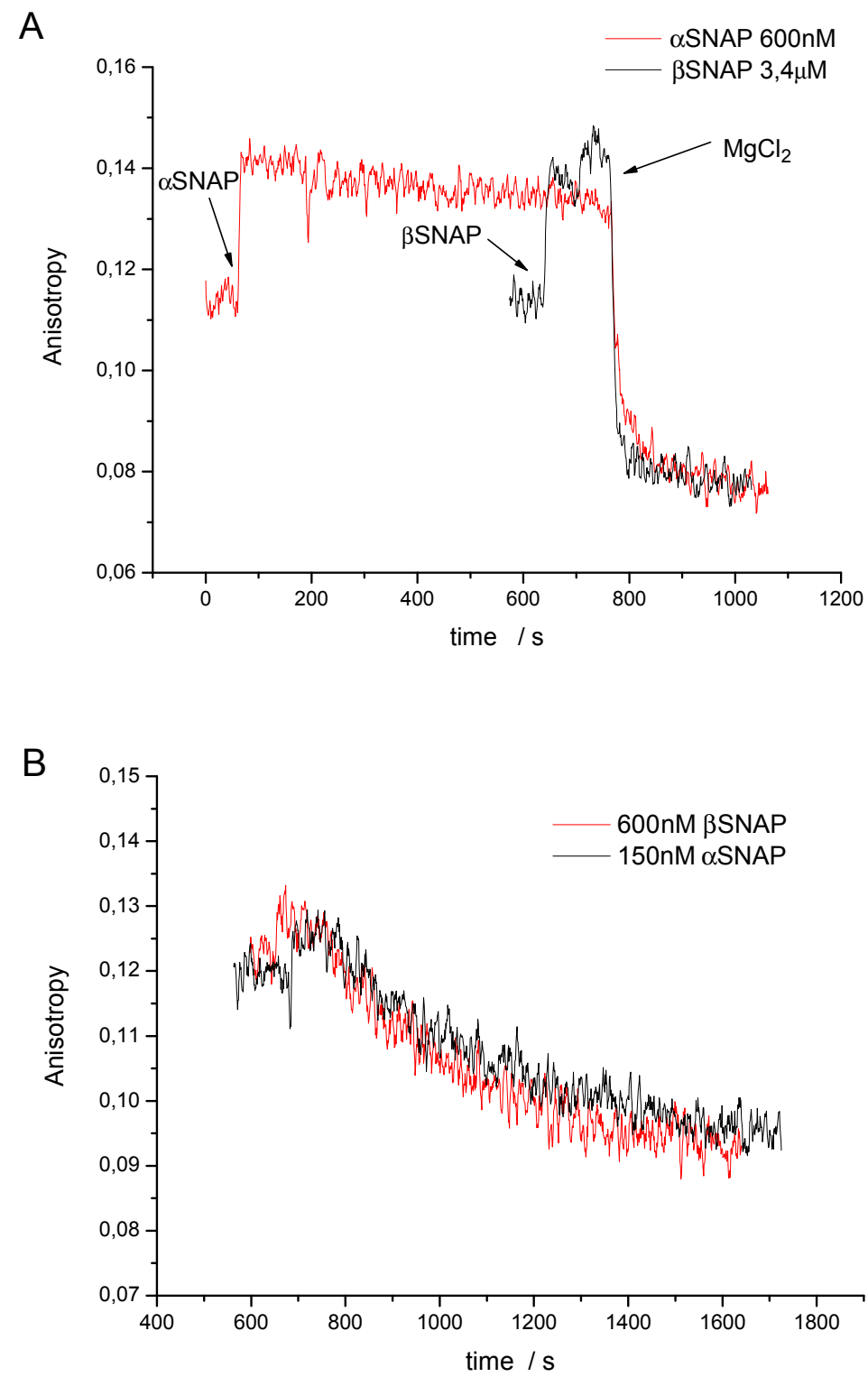

Figure 4.37: $\quad \beta$ SNAP is approximately four times less effective than $\alpha$ SNAP during SNARE-complex disassembly in solution. Anisotropy changes during disassembly of $\sim 70 \mathrm{nM}$ FRET-complex $\left(\mathrm{Sb}^{28 T R} / H 3 / S N A P 25\right)$ at high adaptor-protein concentrations (as indicated in A) and low adaptor-protein concentrations (as indicated in B) with $6 \mathrm{nM}$ NSF are shown. The reactions were triggered by $\mathrm{MgCl}_{2}$ at $780 \mathrm{~s}(\mathrm{~A})$ or $650 \mathrm{~s}(\mathrm{~B})$.

The membrane boost observed for $\alpha$ SNAP is conserved for $\beta$ SNAP To now address the question of whether the potentiation of $\alpha$ SNAP efficiency by incorporation of the complexes into liposomes is conserved for $\beta$ SNAP, disassembly of the two SNAP isoform was compared on liposomes as well. In order to exclude that putative differences between liposomes and solution are caused by different qualities 
of the preparations used, the same preparations of $\alpha$ SNAP and $\beta$ SNAP as in the last experiment were used and reactions carried out on the same day. As illustrated in figure 4.38, $45 \mathrm{nM}$ of $\alpha \mathrm{SNAP}$ disassembled almost as efficiently as $160 \mathrm{nM} \beta \mathrm{SNAP}$. The rates of disassembly in presence of $\alpha$ - or $\beta$ SNAPs are thus comparable when about four times as much $\beta$ SNAP as $\alpha$ SNAP was used like previously observed in solution. The fact that the efficiency-difference between the two SNAPs is the same on liposomes as in solution, indicates that the efficiency of $\beta$ SNAP on liposomes increases to the same extent as has earlier been determined for $\alpha$ SNAP. The positive effect of complex incorporation into liposomes hence is conserved amongst at least two of the SNAP isoforms, namely $\alpha \mathrm{SNAP}$ and $\beta$ SNAP.

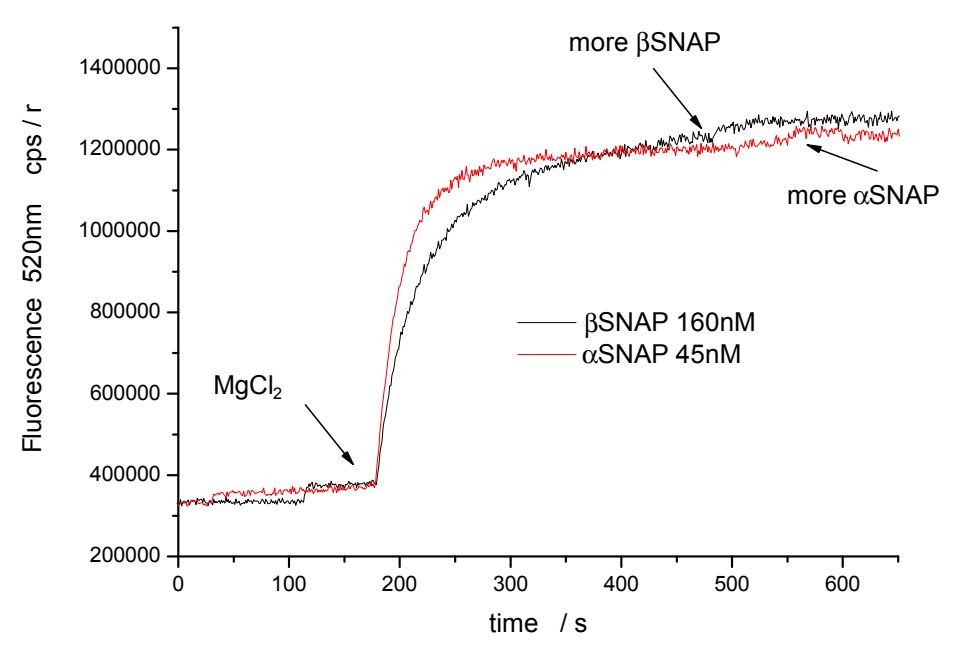

Figure 4.38: $\beta$ SNAP versus $\alpha$ SNAP on liposomes. $160 \mathrm{nM} \beta$ SNAP or $45 \mathrm{nM} \alpha \mathrm{SNAP}$ were pre-incubated with $\sim 70 \mathrm{nM}$ FRET-complex (H3/SNAP25 ${ }^{130 O G} / \mathrm{Sb}^{28 \text { Alexa594}} \mathrm{TMD}$ ), NSF and ATP and the disassembly initiated at $\mathrm{t}=180 \mathrm{~s}$ by $\mathrm{MgCl}_{2}$. 


\subsection{How is the Reaction Being Regulated?}

The newly discovered SNAP potentiation through membrane anchorage now opens up possibilities to investigate putative regulatory mechanisms of SNARE disassembly which act on the level of the SNAP adaptor protein.

\subsubsection{Influence of Complexin1 on Core-Complex Disassem- bly}

Besides SNAP isoforms, putative competitors of $\alpha$ SNAP with respect to SNAREcomplex binding have been suggested, one of these proteins being Complexin1 (Cpx1). Complexin has originally been reported to replace $\alpha$ SNAP from SNARE complexes and meanwhile its crystal structure bound to the SNARE complex has been solved. Gel-based experiments investigating the effect of Complexin on SNARE disassembly have been performed [41] but did not confirm the proposed inhibitory role. Anyhow, as mentioned earlier, such experiments only have a limited time resolution and are thus mainly suited to trace 'all-or-nothing' defects. Therefore, I re-investigated the Complexin influence on SNARE disassembly using the fluorescence assays. First, as shown in figure 4.39, I tested whether Complexin can influence SNARE disassembly in the first place. Even though both, $\alpha$ SNAP and Complexin, were used at very high concentrations compared to the SNARE substrate $(3,6 \mu \mathrm{M}$ and $2 \mu \mathrm{M}$, respectively at $90 \mathrm{nM}$ complex), Complexin was able to slightly inhibit SNARE complex disassembly. The extend of inhibition increased, when the $\alpha$ SNAP concentration was reduced to levels which make $\alpha$ SNAP limiting when disassembling in solution $(1,1 \mu \mathrm{M})$. As a result, the half-time of the reaction increased by a factor of five from 20s to 100s. Complexin was added first, to allow for binding, in all cases.

To now estimate the ratio of Complexin- to $\alpha$ SNAP-concentration at which Complexin is able to modulate $\alpha \mathrm{SNAP}$-function in solution, increasing amounts of $\alpha$ SNAP were titrated into a FRET complex under disassembly conditions, either in presence or absence of 150nM Complexin1 (data not shown). Comparison of the two resulting donor spectra indicated that the sample containing Complexin was less efficiently disassembled at equal $\alpha$ SNAP concentrations up to an $\alpha$ SNAP concentration of $625 \mathrm{nM}$. A more than four-fold excess of $\alpha \mathrm{SNAP}$ was hence needed to fully compensate the inhibition. Figure 4.40 illustrates that 370nM Complexin are sufficient to strongly inhibit SNARE disassembly at an $\alpha$ SNAP concentration of $1,1 \mu \mathrm{M}$. 

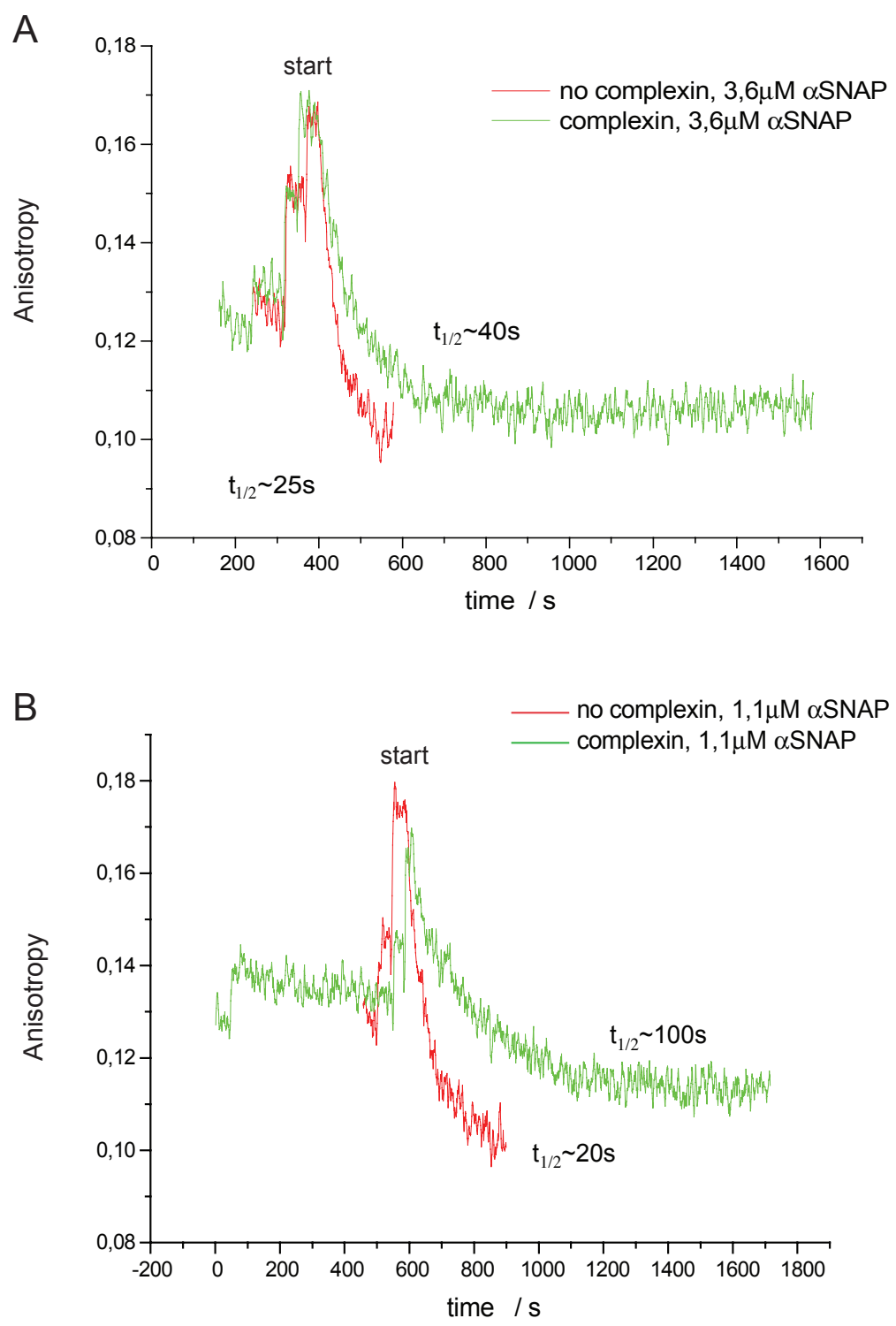

Figure 4.39: Influence of Complexin on core complex disassembly. Fluorescence anisotropy was recorded during SNARE-complex disassembly (90nM of purified SNAREcore complex labelled with Texas red at $\mathrm{Sb}^{\text {cys28 }}$ ) either with or without $2 \mu \mathrm{M}$ of Complexin1 (1-134). 3,6 $\mu \mathrm{M}$ (A) versus $1,1 \mu \mathrm{M}$ (B) of $\alpha \mathrm{SNAP}$ are compared. Proteins were added as follows: (A) Complexin was added after 240s, and can be seen as a small increase in the green graph. Subsequently, $\alpha$ SNAP was added to both reactions at 320 s. Note that both reaction show a similar increase, independent of whether they contain Complexin or not. Finally NSF was added at $380 \mathrm{~s}$ and $\mathrm{MgCl}_{2}$ at $420 \mathrm{~s}$. (B) Complexin was added after $40 \mathrm{~s}$, $\alpha$ SNAP after $520 \mathrm{~s}$, NSF after 580 s in the red graph; $\alpha$ SNAP at 500 s and NSF at 550 s in the red graph. The reactions were triggered by $\mathrm{MgCl}_{2}$ at $620 \mathrm{~s}$. 


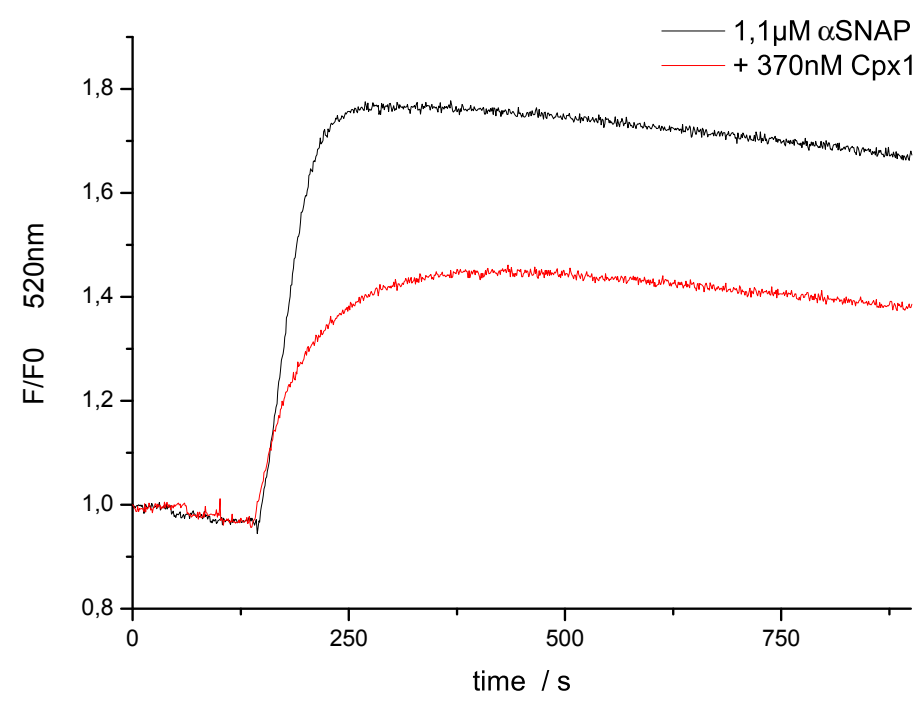

Figure 4.40: Complexin is dominant over $\alpha$ SNAP in FRET experiments in solution. Disassembly at $1,1 \mu \mathrm{M} \alpha \mathrm{SNAP}$ was triggered after $140 \mathrm{~s}$ in presence or absence of $370 \mathrm{nM}$ Complexin. The reactions included $4,5 \mathrm{nM}$ NSF and $\sim 75 \mathrm{nM}$ complex (H3/SNAP2 $\left.5^{130 O G} / \mathrm{Sb}^{28 T R}\right)$.
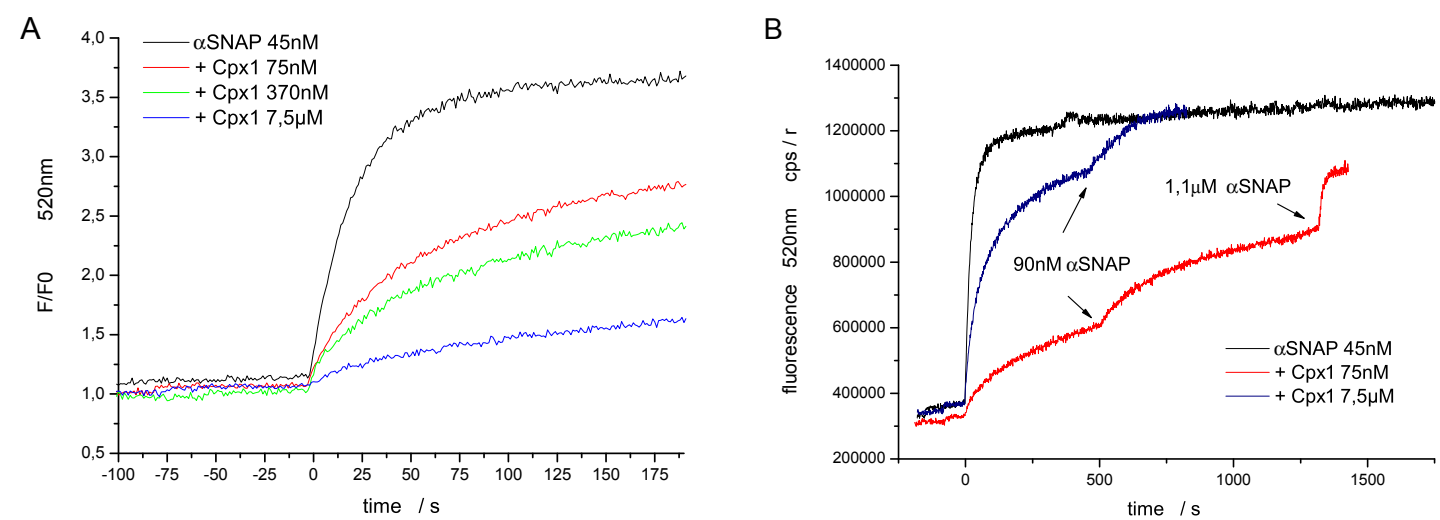

Figure 4.41: Influence of Complexin on disassembly of liposomal complexes. FRET spectra of SNARE complex (H3/SNAP2 $5^{130 O G} / \mathrm{Sb}^{28 T R}$-TMD) disassembly were recorded at $45 \mathrm{nM} \alpha \mathrm{SNAP}$ and various concentrations of Cpx1.

If $\alpha$ SNAP and Complexin really were to compete in the cell, performing competition experiments in presence of the membrane would naturally be more reasonable. These might lead to a completely different outcome than in solution, depending on whether the membrane influences Complexin's efficiency during disassembly in a similar way as $\alpha$ SNAP or not. An additional advantage of investigating the Complexin/SNAP interplay on liposomes is the possibility to employ stoichiometric amounts of $\alpha$ SNAP. As pointed out earlier, this is not possible in solution experiments, because $\alpha$ SNAP needs to be employed in a $\mu \mathrm{M}$ range even at low substrate 


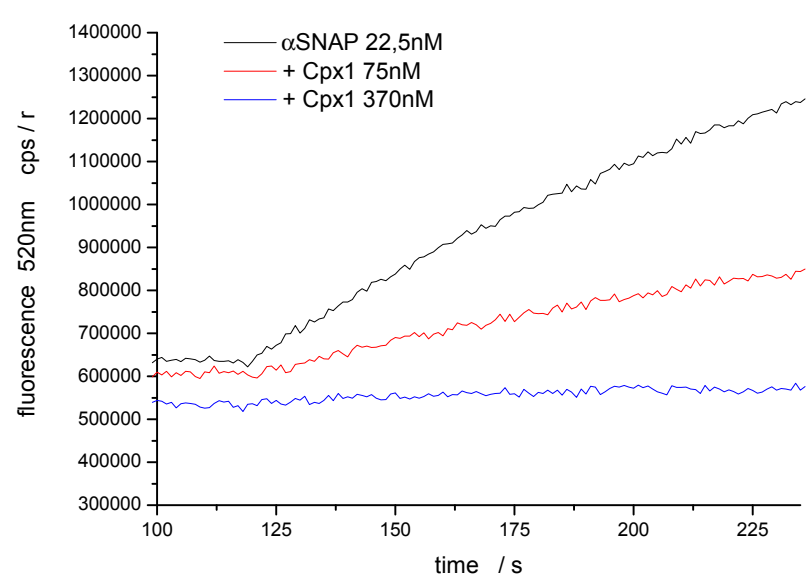

Figure 4.42: Full block of disassembly via an excess of Complexin at very low amounts of $\alpha$ SNAP $(22,5 \mathrm{nM})$. The donor fluorescence of FRET experiments looking at disassembly of $22,5 \mathrm{nM} \alpha \mathrm{SNAP}$ in presence of various amounts of $\mathrm{Cpx} 1$ is shown. All reactions were mediated by $5 \mathrm{nM}$ of $\mathrm{NSF}$ and triggered by $\mathrm{MgCl}_{2}$ at $120 \mathrm{~s}$. Note that disassembly is blocked completely at $370 \mathrm{nM}$ of Complexin.

concentration in order to function efficiently. Hence, competition experiments were also performed on liposomes as shown in figure 4.41. To this end, SNARE complexes were incorporated into liposomes and disassembled in the presence or absence of Complexin1. Here, $75 \mathrm{nM}$ of Complexin partially inhibited disassembly mediated by $45 \mathrm{nM} \alpha \mathrm{SNAP}$, and $7,5 \mu \mathrm{M}$ of Complexin inhibit disassembly almost completely. Nevertheless, the inhibition by $75 \mathrm{nM}$ Cpx1 is overcome by raising the $\alpha \mathrm{SNAP}$ concentration to $90 \mathrm{nM}$. Even the more pronounced inhibition caused by $7,5 \mu \mathrm{M}$ of $\mathrm{Cpx} 1$ is partially recovered by $90 \mathrm{nM} \alpha \mathrm{SNAP}$ and almost completely relieved when $\alpha \mathrm{SNAP}$ concentration is raised to $1,1 \mu \mathrm{M}$.

Interestingly, Complexin completely blocked SNARE disassembly when very low amounts of $\alpha \mathrm{SNAP}$ were used $(22,5 \mathrm{nM})$ as illustrated in figure 4.42 . Yet, this block mediated by 370nM Cpx1 was partially relieved, as soon as the $\alpha$ SNAP concentration was increased to $45 \mathrm{nM}$ (A). Interestingly, the extent and the rate of this reaction are identical to the rate in presence of $75 \mathrm{nM} \mathrm{Cpx} 1$ and $22,5 \mathrm{nM} \alpha \mathrm{SNAP}$, maybe indicating that the extend of inhibition is confined to discrete levels. On the contrary to what has been observed in solution, 450nM $\alpha$ SNAP (in other words a $30 \%$ excess of $\alpha \mathrm{SNAP}$ with respect to Complexin) then suffice to completely relieve the block. A significantly higher ratio of Complexin: $\alpha$ SNAP is hence needed on liposomes than in solution, to successfully impair disassembly. 

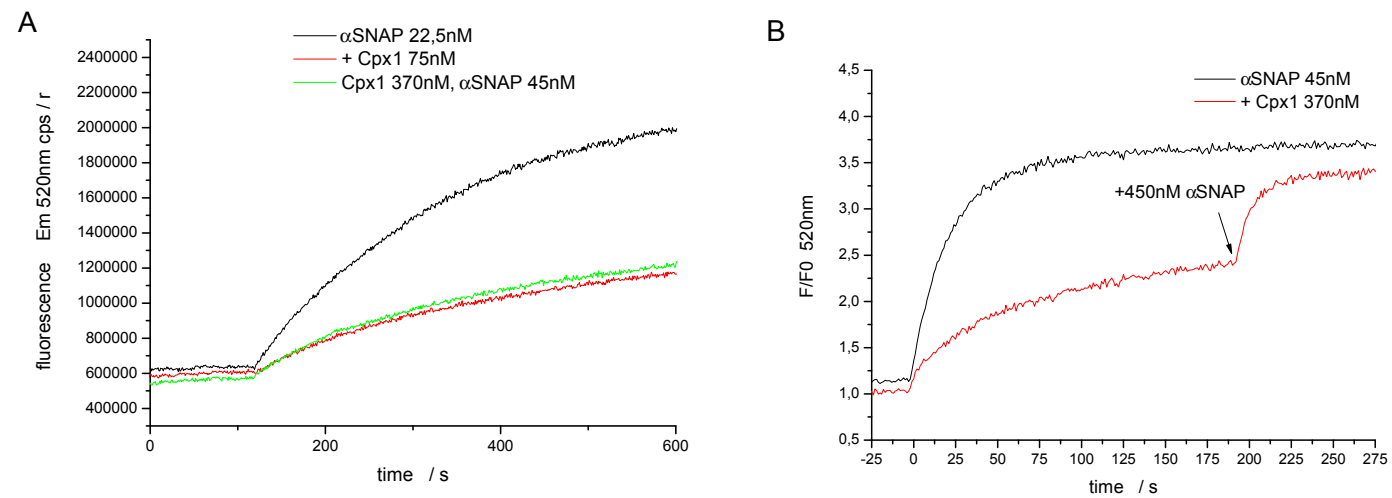

Figure 4.43: $\alpha$ SNAP is dominant over Complexin on liposomes. FRET SNARE-complex and NSF concentration are identical to figure 4.42, the $\mathrm{Mg}^{2+}$-trigger was added after 130s. (A) Complexin/ $\alpha$ SNAP ratios of 3:1 (75nM Cpx:22,5nM $\alpha$ SNAP) and 6:1 (370nM Cpx:45nM $\alpha$ SNAP) inhibit disassembly to the same extent. (B) Addition of $450 \mathrm{nM}$ $\alpha$ SNAP at $\mathrm{t}=200$ s to the reaction containing $370 \mathrm{nM}$ Cpx1 completely relieves the inhibition.

Altogether, the data suggest that $\alpha$ SNAP function is not as fragile with respect to Cpx1 as in solution, where even a 3-fold excess of $\alpha$ SNAP could not recover normal disassembly speeds. This is probably due to the fact that much lower amounts of $\alpha$ SNAP are needed for disassembly on membranes compared to solution. The affinity of Complexin hence does not seem to vary between liposomal and soluble complexes. Complexin therefore has a selective advantage over $\alpha$ SNAP in solution.

\subsubsection{Does Phosphorylation of NSF Have an Impact on Func- tion?}

The competition between $\alpha$ SNAP and Complexin discussed in the last section affects the interface between the SNAPs and the SNARE complex. An additional level of regulation is feasible at the interaction sites between the SNAPs and NSF. For example, phosphorylation of NSF at a tyrosine at position 83 has been reported to reduce NSF's affinity for $\alpha$ SNAP [36]. This hypothesis is best to be tested under conditions which allow for the use of limiting amounts of $\alpha$ SNAP, making the fluorescence assays on liposomes ideally suited.

To this end, two NSF mutants were expressed and purified: First, a wildtype construct of NSF was expressed in an E. coli strain in which proteins can be in vitrophosphorylated directly inside the bacteria during expression. The strain contains a second plasmid coding for a kinase whose expression is under control of a tryptophane operon and can hence be induced independently. Expression of the kinase is triggered by changing the expression medium to a medium containing indole-acrylicacid (IAA) as an inducing agent after three hours of NSF production. The kinase then phosphorylates NSF which can afterwards be purified according to the protocols used for unphosphorylated NSF. Phosphorylation was subsequently confirmed 
by Western blotting (data not shown). Using this approach the degree of phosphorylation can however not be determined. Therefore, we also employed a second approach to have a control which should behave like NSF phosphorylated to saturation: A phosphomimetic point-mutant $\left(\mathrm{NSF}^{Y 83 E}\right)$ of NSF was expressed, which was purified according to the protocols for wildtype NSF as well. During purification of $\mathrm{NSF}^{Y 83 E}$ it became evident that the monomeric peak was relatively higher, whereas the hexameric was relatively smaller compared to wildtype NSF preparations (data not shown). This may have a physiological background, but could as well simply be due to an increased fragility of the mutant under the conditions used during purification.

As shown in section 4.1.2, the phosphomimetic mutant indeed performed less efficient than wtNSF in the gel-based disassembly assay. However, as mentioned in section 4.1 .2 as well, this approach does not allow to differentiate between incomplete disassembly and partial re-assembly. The interpretation of such a finding is thus more difficult than in the 'real-time' fluorescence approach.

A

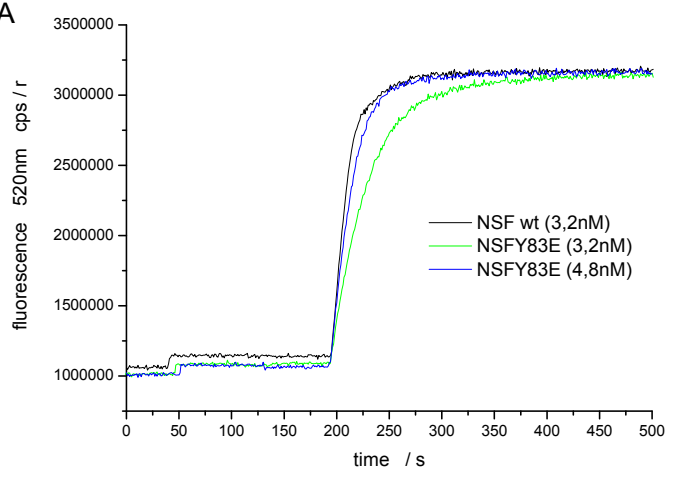

Figure 4.44: $\quad \mathrm{NSF}^{Y 83 E}$ efficiently

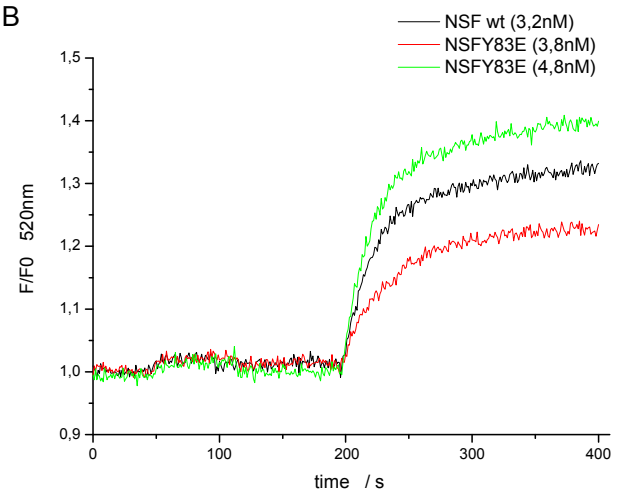
disassembles liposomal complexes
liposomes via the Sb-TMD (A) or liposomes via the Sb-TMD (A) or the H3-TMD (B), respectively. FRET complexes were disassembled using 60nM $\alpha$ SNAP and $\mathrm{NSF}$ or $\mathrm{NSF}^{Y 83 E}$ as indicated. The reaction was triggered by $\mathrm{MgCl}_{2}$ after $180 \mathrm{~s}$.

Consequently, both of these mutants were tested in the FRET assay, where they behaved similarly. Therefore, disassembly on liposomes is exemplified only for $\mathrm{NSF}^{Y 83 E}$ (figure 4.44). Liposomal SNARE complexes were incorporated either via the $\mathrm{Sb}$ - or the H3-transmembrane domain and afterwards disassembled by either wildtype NSF or $\mathrm{NSF}^{Y 83 E}$. For both complexes the mutant performed slightly less efficient than the wildtype. In case of the Sb-TMD complex, disassembly was only slightly slowed down whereas for the H3-TMD complex, only half of the complex was disassembled by the mutant at all. Increasing the mutant's concentration by $50 \%$ anyhow led to disassembly comparable to, or in case of the H3-TMD complex even better than, the wildtype indicating that the difference was not very pronounced.

On liposomes, the differences between wildtype and phosphorylated NSF can be diminished by increasing the mutant's concentration by $50 \%$, a difference which 
might as well result from reduced stability of the mutant. At this stage it should again be pointed out that NSF is known to be a fragile enzyme and it is difficult to compare the preparations of the wildtype and the point mutation quantitatively, since it can never be excluded that differences between the enzymes stem from buffering conditions which, even though optimized for NSF as described in section 4.1.3, do not mimic the enzymes natural environment.

To next investigate, whether the reported phosphorylation-mediated defect of NSF function is dependent on the Habc domain of Syntaxin, the experiments were also performed with liposomal complexes containing full-length Syntaxin, again once incorporated via the Sb- and once via the Syntaxin-transmembrane domain. Again, using 1,5-fold the amount of mutant sufficed to disassemble at comparable speeds as the wildtype, indicating that the Sx Habc domain does not influence the reaction (see figure 4.45).
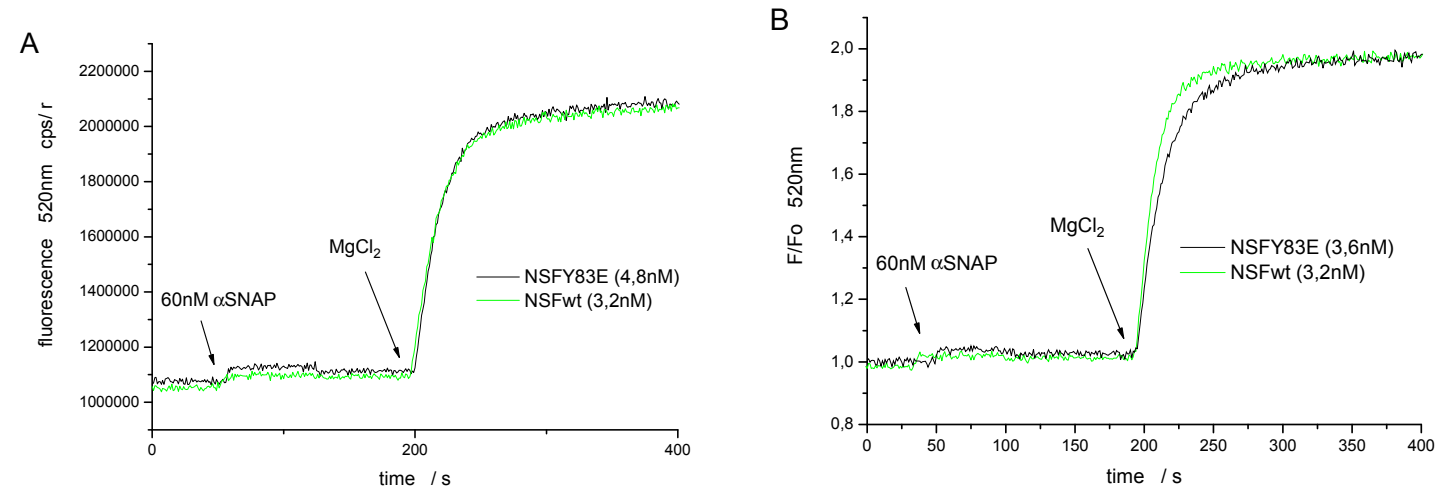

Figure 4.45: $\mathrm{NSF}^{Y 83 E}$ efficiently disassembles liposomal complexes containing full-length Syntaxin. Everything was carried out as in figure 4.44 except that complexes contained full-length Syntaxin (including the Habc domain) (Sx/SNAP25 $5^{130 O G} / \mathrm{Sb}^{28 T R}$ ) (A) or the Sx-TMD (B), respectively.

To clarify, whether the disassembly differences observed in the gel-based assay only show up in solution, the FRET assay was carried out in solution next. As shown in figure 4.46, phosphorylated NSF once more disassembled slower and less efficiently than wildtype when used at identical concentrations. As opposed to the liposomal findings however, this deficiency could not be overcome by adding more phosphorylated NSF. Here, it is therefore less likely that the imperfect disassembly by pNSF is due to a lower concentration or stability. Due to the reported defect in $\alpha$ SNAP binding, I subsequently tested whether disassembly would improve in presence of more $\alpha$ SNAP. Indeed, the defect is almost completely abrogated by increasing the $\alpha \mathrm{SNAP}$ concentration from $1,1 \mu \mathrm{M}$ to $2,2 \mu \mathrm{M}$. Whether this is due to a reduced $\alpha \mathrm{SNAP}$ affinity of $\mathrm{NSF}^{Y 83 E}$ in solution remains to be elucidated. Further experiments hence need to be performed in order to get further insights into whether the phosphorylation indeed leads to a mechanistical difference of the mutant or whether the mutant is simply less stable in my hands than the wildtype. 


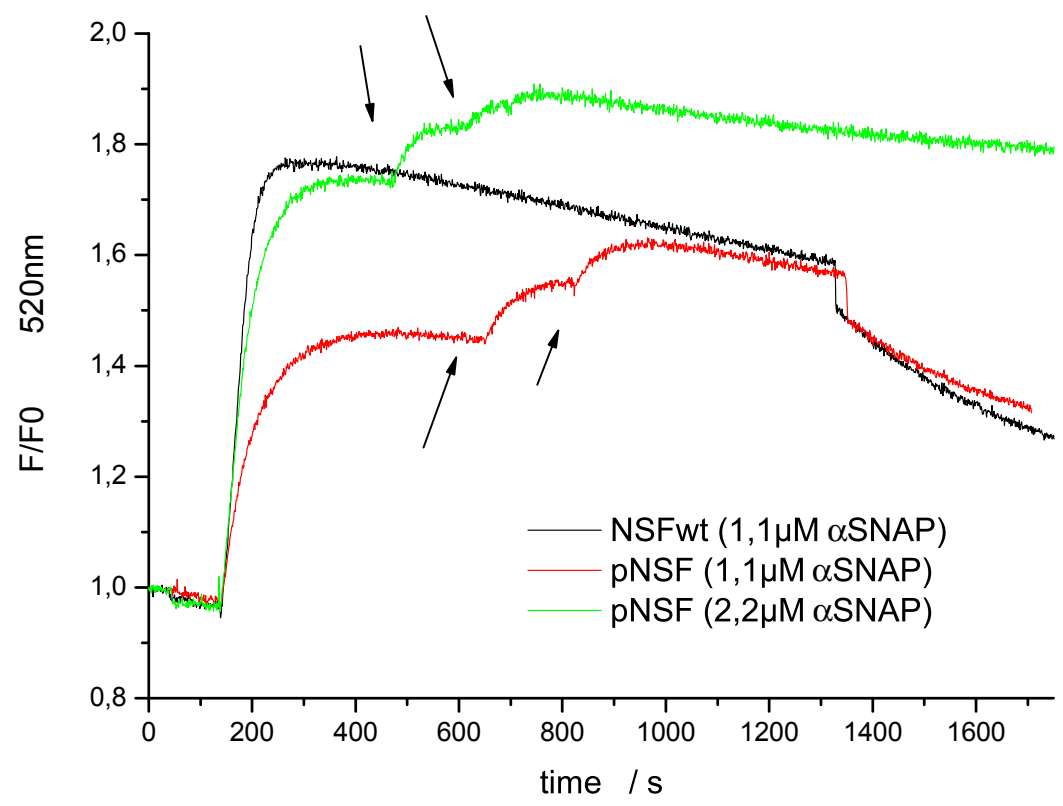

Figure 4.46: pNSF performance at $1,1 \mu \mathrm{M} \alpha \mathrm{SNAP}$ is less efficient in solution. Everything was carried out as in figure 4.44 except that soluble complexes and NSF and $\alpha$ SNAP as indicated were used. The reaction was triggered by $\mathrm{MgCl}_{2}$ after 130 s. The Black arrows indicate further additions of pNSF after equilibrium has been reached. 


\subsection{How Conserved is the Reaction Mechanism?}

\subsubsection{SNARE Disassembly Using Yeast Homologues}

The fact that $\alpha$ SNAP and NSF are ubiquitously expressed in all tissues and mediates the recycling of most if not all intracellular SNARE proteins involved in intracellular fusion processes suggests that the mechanism of the different disassembly reactions throughout the cell is strongly conserved. Considering that the four-helix-bundle structures of the different SNARE complexes show a high degree of similarity, the overall sequence homology between the different complexes studied thus far is surprisingly low. It is hence possible that the SNAP/SNARE machinery does not care about conservation of specific residues but rather recognizes its target proteins on the basis of their quaternary structure. To elucidate, whether even between two organisms as distant as yeast and mammals the degree of conservation is high enough to provide functionality of one machinery in the other organism, components of the mammalian machinery were tested on yeast SNARE complexes and vice versa. Subsequently disassembly experiments were performed using various combinations of the yeast and the mammalian components, to determine which of the parts or interfaces are especially conserved and whether the mechanisms found for the neuronal SNARE complex (as e.g. the 'membrane boost') are conserved in yeast.

\subsubsection{Disassembly of Neuronal Complexes Using the Yeast Machinery}

To this end, the yeast homologues of $\alpha$ SNAP and NSF (Sec17 and Sec18, respectively) were expressed and purified according to the protocols used for their mammalian counterparts. Unfortunately, Sec18 activity could not be recovered after gel filtration. Furthermore, the ratio between monomeric and hexameric Sec18 after gel filtration varied drastically between preparations and was shifted towards monomeric enzyme with respect to the average NSF preparation. This could indicate that Sec18 is either less stable than NSF under the purification conditions used or that the equilibrium is naturally shifted to the monomeric (i.e. inactive) fraction in case of Sec18. Anyhow, since activity was not recovered after gel filtration, Sec18 was directly used after Ni/NTA-chromatography for all of the following experiments. Sec17 did not express well and still contained impurities after purification. Furthermore, it soon became evident that both, Sec17 as well as Sec18, tend to loose activity very rapidly (in a day or two from purification). Sec17 was therefore also employed directly after elution from the Ni-beads, omitting the second chromatographic step. All experiments using the yeast machinery, therefore do not aim to determine absolute activities or rates of disassembly. Even though the true efficiency of the yeast machinery can not be determined that way, qualitative studies can be carried out. As will be shown in the next section, some questions regarding the degree of conservation of the disassembly mechanism can be resolved merely on the basis of comparative measurements. 


\section{'Component Swapping' between the yeast and the mammalian reaction}

First, all possible combinations $(\alpha \mathrm{SNAP} / \mathrm{NSF}$, Sec17/Sec18, $\alpha \mathrm{SNAP} / \mathrm{Sec} 18$ and Sec17/NSF) were tested for disassembly neuronal SNARE complexes. $\alpha$ SNAP and NSF were most efficient, followed by the complete yeast machinery (Sec17 and Sec18) and the combination of Sec18 and $\alpha$ SNAP, which worked equally efficient. As mentioned above, this does not allow to draw the conclusion that the yeast machinery is less efficient than the mammalian in general, but what can be seen from this experiment is that the yeast machinery is capable of disassembling neuronal complexes. Furthermore, the efficient disassembly mediated by $\alpha$ SNAP and Sec18 indicates that the interface and basic mechanism on the level of the adaptor-enzyme interplay seems to be conserved (figure 4.47). To ensure that Sec17 is not limiting, a second reaction (data not shown) using a four-fold excess of Sec17 was performed, which led to the same kinetics. What is also striking in this experiment is the fact that the combinations $\operatorname{mammalian}_{\text {complex }} / \operatorname{mammalian}_{\text {adaptor }} /$ yeast $_{\text {enzyme }}$ and $\operatorname{mammalian}_{\text {complex }} /$ yeast $_{\text {adaptor }} /$ yeast $_{\text {enzyme }}$, both containing one interface between non-cognate proteins, display similar kinetics. Interestingly, the combination of Sec17 and NSF did not promote disassembly of the neuronal complex at all, even though used at the same concentrations as in the other combinations which all led to efficient disassembly (see figure 4.47). Spontaneously, two scenarios are feasible: Either the interface between Sec17 and NSF has diverged so much during evolution that the two cannot productively interact and disassemble at all. Alternatively, the connection could only be weakened but still be able to function, as long as the cognate substrate for Sec17 were provided. This would result in a combination containing only one interface between non-cognate proteins and might successfully disassemble like the other combinations with one incorrect interface. When disassembling the non-cognate neuronal complex however, disassembly might already be hampered by an imperfect interaction between Sec17 and the neuronal complex and then completely be abolished by the second impeded interaction between the non-cognate adaptor and enzyme.

\section{The membrane boost is conserved for the yeast adaptor protein Sec17}

To now investigate, whether the membrane-caused potentiation of efficiency found for $\alpha$ SNAP is conserved for the yeast adaptor protein Sec17, further experiments using more Sec17 were carried out, in order to see whether or not the combination of Sec17 and NSF could not be brought to function at least partially. If this were to be the case, the same amounts of Sec17 to NSF could be applied to disassemble SNARE complexes in solution, and the kinetics compared. Indeed, the combination of Sec17 and NSF mediated slow and partial disassembly when used at high amounts. Using the same amounts afterwards to disassemble soluble complexes was not successful (Figure 4.48). It can thus be concluded that Sec17 efficacy and in analogy to $\alpha$ SNAP probably affinity are higher when the target complexes are incorporated into membranes. 


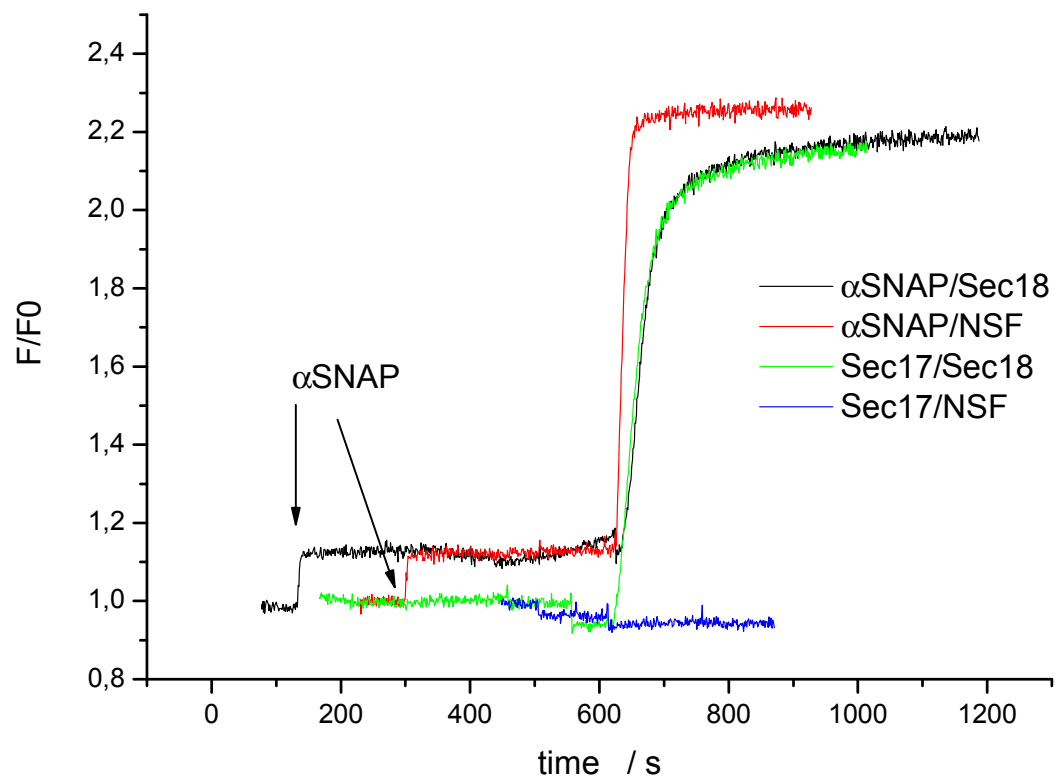

Figure 4.47: All except one combination of the yeast and the mammalian disassembly machinery efficiently disassemble neuronal SNARE complexes. Approx. 40nM of SNARE complexes $\left(\mathrm{H} 3 / \mathrm{Sb}^{28 T R} \mathrm{TMD} / \mathrm{SNAP} 25^{130 O G}\right)$ were disassembled with the adaptor/enzymepairs as indicated. Concentrations used were $600 \mathrm{nM}$ for $\alpha$ SNAP, $6 \mathrm{nM}$ NSF and roughly estimated (from the gel) to be $350 \mathrm{nM}$ for Sec17 and 200nM for Sec18 after Ni/NTA-elution (hence still including the monomeric fraction). The reaction was triggered by $\mathrm{MgCl}_{2}$ at $630 \mathrm{~s}$. The arrows indicate $\alpha \mathrm{SNAP}$ addition which results in a small increase of donor signal. This rise is not observed for the addition of Sec17. 


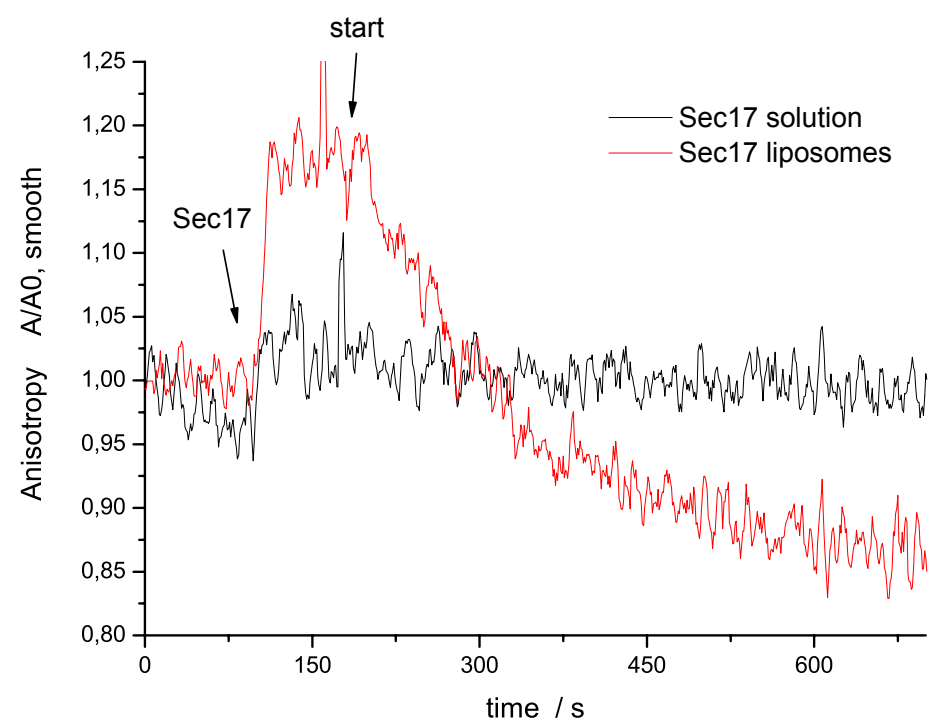

Figure 4.48: The 'imperfect' adaptor protein Sec17 also shows membrane dependence. Roughly 700nM Sec17 (as estimated from the gel) and 1,5nM NSF were used to disassemble $40 \mathrm{nM}$ of liposomal SNARE complexes (H3/Sb $\left.{ }^{28 T R} \mathrm{TMD} / \mathrm{SNAP} 25^{130 O G}\right)$. The time point of Sec17-application as well as the start of the reaction by addition of $\mathrm{MgCl}_{2}$ are indicated. 


\subsubsection{Disassembling the Yeast SNARE Complex}

As stated in the last section 4.6.2, recombinant Sec17 and Sec18 are less efficient in disassembling the neuronal SNARE complexes than NSF and $\alpha$ SNAP. Considering the fact that their activities are also less reproducible between preparations than those of the mammalian machinery, the lower efficiency is presumably at least partially caused by a lower intrinsic activity. This might be improvable by further optimization of the purification protocols. Nevertheless it is impressive that the yeast machinery disassembles the neuronal complex at all, considering that this do not represent its cognate target. It would hence be interesting to know, whether the yeast machinery performs better when disassembling yeast SNARE complexes. Likewise the question arises, whether the target recognition of the mammalian machinery is robust enough to disassemble yeast SNARE complexes as well. To address these questions, yeast SNARE complexes were assembled and subsequently disassembled by either the mammalian or the yeast disassembly machinery. The yeast SNAREs Sec9 (residues 403-651), Sec9 $9^{c y s 587 T R}$, Snc2 (residues 1-93) with and without TMD, Snc2 ${ }^{c y s 24}$ as well as Sso1 (residues 179-264) with and without TMD were expressed, purified and kindly provided by Xiong Chen.

\section{The machineries do not preferentially disassemble their cognate com- plexes}

As illustrated in figure 4.49, both machineries are able to disassemble both complexes. Again, as has been observed for the neuronal complex earlier, the yeast machinery disassembles less efficiently than the mammalian, even when it comes to disassembling its cognate complex. Whether this is only due to a lower stability in the purification buffers or also has a physiological meaning remains unclear at this point. Interestingly, the kinetics do not vary significantly with respect to whether the respective cognate or non-cognate SNARE complex is disassembled. From this one can deduce that SNARE-complex recognition by the disassembly machinery indeed is either very robust or very conserved between yeast and mammals.

\section{'Component swapping'}

Finally, the disassembly components were also 'swapped' as previously shown for the disassembly of the neuronal SNARE complexes. Knowing from section 4.6.2 that Sec17/NSF do not perform well together when disassembling neuronal complexes, using the yeast SNAREs opens up the possibility to further elucidate the reasons for this incompatibility. If Sec17 and NSF simply do not fit together, they would be expected to perform least efficiently of all combinations during yeast-complex disassembly as well. If on the other hand the reason for their imperfect performance is due to a synergistic defect caused by two non-cognate binding sites as discussed in section 4.6.2, they would be expected to disassemble yeast SNARE complexes more efficiently. 


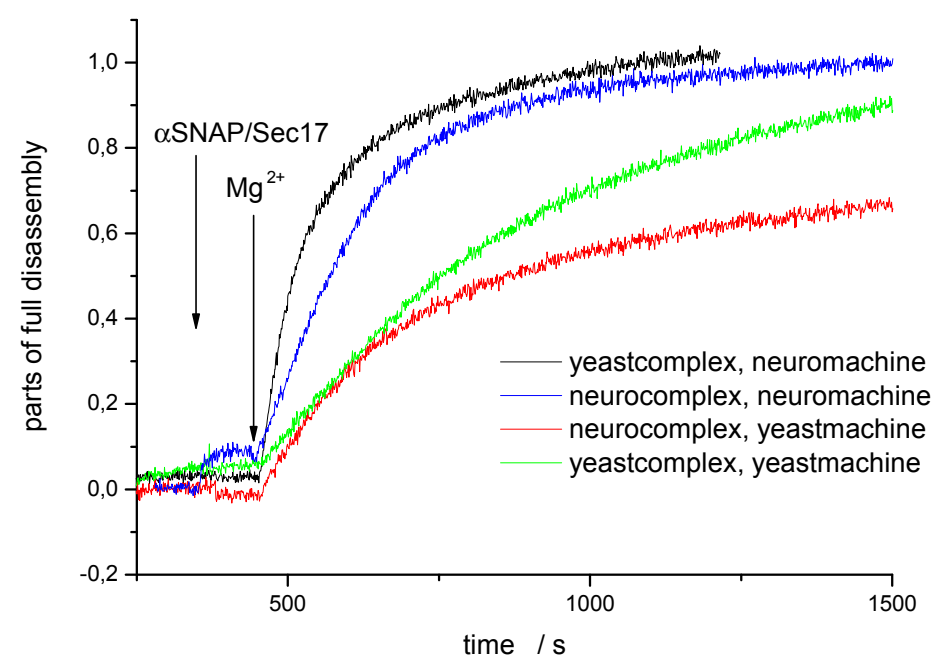

Figure 4.49: Disassembly of yeast vs. neuronal SNARE complexes. Approximately $40 \mathrm{nM}$ of either yeast (Sec9 $5780 G / \mathrm{Snc} 2^{24 T R} / \mathrm{Sso1TMD}$ ) or neuronal (SNAP25 ${ }^{130 O G} \mathrm{Sb}^{28 T R} \mathrm{TMD} / \mathrm{H} 3$ ) SNARE complex were disassembled by the machinery as indicated. $90 \mathrm{nM}$ of $\alpha \mathrm{SNAP}$ and $\sim 3 \mathrm{nM}$ of NSF were used and the reaction triggered at $450 \mathrm{~s}$ as marked. The amounts of yeast machinery used were identical between the reactions and roughly estimated (by appearance on the gel) to be $250 \mathrm{nM}$ for Sec17 and 100nM for Sec18 (still including monomeric fraction).
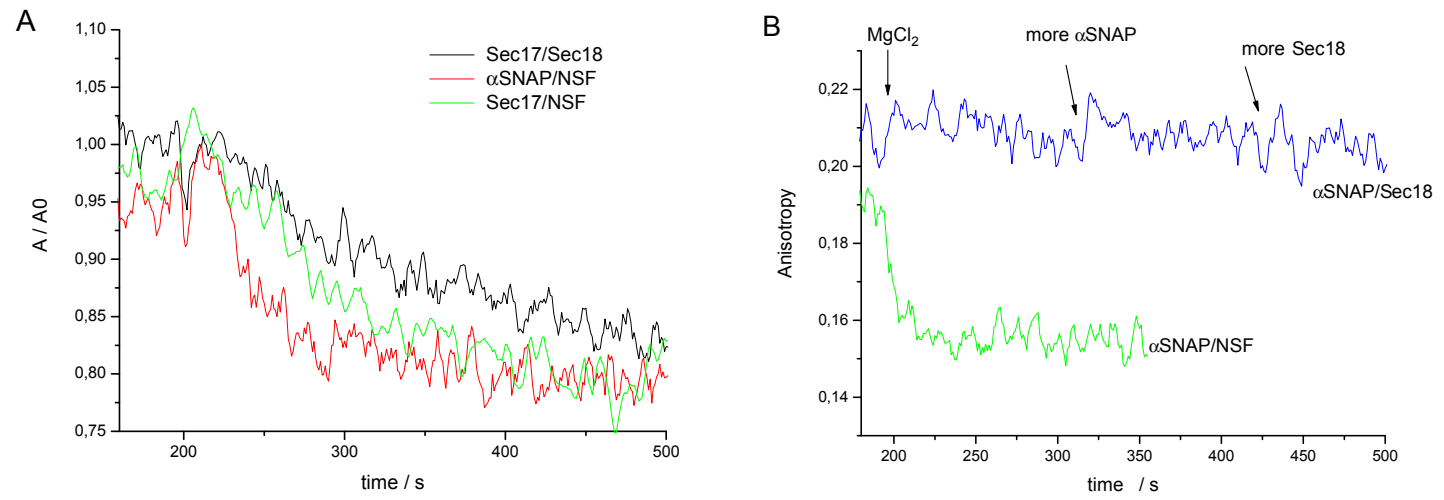

Figure 4.50: All except one combination of the yeast- and the mammalian disassembly machinery efficiently disassemble yeast SNARE complexes. (A) Anisotropy changes upon disassembly of $\sim 40 \mathrm{nM}$ of yeast SNARE complexes (Snc2TMD/Sec $9^{T R} / \mathrm{sso} 1 \mathrm{p}$ ) with the adaptor/enzyme-pairs as indicated. Concentrations used were 120nM for $\alpha \mathrm{SNAP}, 6 \mathrm{nM}$ NSF and roughly estimated to be $350 \mathrm{nM}$ for Sec17 and 100nM for Sec18 from Ni elution (hence still including the monomeric fraction). The reaction was triggered by $\mathrm{MgCl}_{2}$ at 220 s. (B) $\sim 40 \mathrm{nM}$ of yeast SNARE complexes ( $s s 01 \mathrm{pTMD} / \mathrm{Sec} 9 / \mathrm{Snc} 2^{T R}$ ) were disassembled by $120 \mathrm{nM} \alpha$ SNAP and either $6 \mathrm{nM}$ NSF or 100nM Sec18 as indicated.

As shown in figure 4.50, the second theory seems to be true. Sec17/NSF medi- 
ated yeast disassembly is not severely hampered during yeast-complex disassembly. On the contrary, they rather efficiently disassemble the yeast SNARE complex like the combinations Sec17/Sec18 and $\alpha$ SNAP/NSF (4.50). Strikingly, the combination of $\alpha$ SNAP and Sec18 however does not mediate disassembly of the yeast complex under the same conditions used in the other experiments. Note that this combination (yeast complex $/ \operatorname{mammalian}_{\text {adaptor }} /$ yeast $_{\text {enzyme }}$ ) correlates to the least efficient combination in the last section ( $\operatorname{mammalian}_{\text {complex }} /$ yeast $_{\text {adaptor }} / \operatorname{mammalian}_{\text {enzyme }}$ ) in the sense that both contain two interfaces between non-cognate proteins. In summary it can be concluded that both of the protein interfaces between target, adaptor and enzyme seem to be conserved well enough between yeast and mammals to allow proteins of the respective other organism to mediate disassembly. Notwithstanding the fact that disassembly occurs even with exchanged machineries, the kinetics of disassembly may be slightly impeded by two non-cognate proteins at any of the interaction interfaces and are almost completely abolished when both interactions are mediated by non-cognate proteins. 


\section{Chapter 5}

\section{Discussion}

The aim of this study was to investigate the molecular basis of SNARE disassembly including SNAP homologues, putative regulatory factors and the degree of functional conservation.

Since its discovery almost 30 years ago, surprisingly little progress has been made in deciphering the molecular details of NSF's action in the cell. In part, this may be due to the fact that its function was originally misinterpreted to be the completion of fusion as such [68, 69, 70]. Only years later did it become evident that it is the SNARE assembly which leads to membrane merger, whereas the role of NSF is the recycling of dead-end SNARE-complexes after fusion instead. Nevertheless, its catalytic activities provide the fuel for intracellular membrane fusion by ATPhydrolysis. NSF hence functions as the motor of membrane fusion, even though its activity is temporally uncoupled from membrane merger. The disassembly reaction thus bears a high degree of fascination, again leading to the question of why it has not been studied more extensively.

One reason probably is that the most efficient approach to get direct molecular insights into time-dependent protein interactions, such as enzymatic reactions, is to look at them using in vitro systems. A prerequisite for such studies is the availability of recombinant proteins. NSF however, being an enzyme, needs to retain its activity during isolation and handling to allow for its use in quantitative functional studies. Due to its fragility, its activity is easily lost under various buffering conditions. This difficulty adds onto the usual demands of protein purification, making it an especially challenging protein to be produced recombinantly.

A second difficulty when working with fragile enzymes, is to find easy ways of judging whether the enzyme is reproducibly active at the time of experiment or not. A read-out of disassembly which is simple enough to allow for direct conclusions about NSF-activity opens up this possibility. We therefore employed the strategy of developing time-resolved assays for NSF-mediated disassembly and used these for quality control of NSF already during various purification stages in order to help optimization of NSF purification. Since fluorescence spectroscopy of SNARE proteins is well established in our lab, it was the most obvious option to also employ this technique for the investigation of their disassembly. To this end, fluorescence- 
based experimental read-outs were established, which can directly monitor SNAREcomplex interactions under disassembly conditions in a time-resolved manner.

During the time of my thesis, a second fluorescence-based in vitro assay using SNAREs fused to GFP-analoga as substrates was published $[62,67]$. The authors successfully used the method to contradict previously described SNARE disassembly defects, which had been claimed to be caused by mutations in the SNARE-complex 0-layer $[64,65]$. In the hands of Lauer et al., core SNARE complexes mutated in the 0-layer were disassembled equally well as wildtype SNARE core complexes in a time scale of seconds. Surprisingly, even though the amounts of NSF used were reasonably low for enzymatic reactions, the EC50 of $\alpha$ SNAP with respect to SNARE complex binding was $5 \mu \mathrm{M}$, which is unexpectedly high considering that the complex concentration was only 100nM. Such low $\alpha$ SNAP-affinities have also been observed by other groups pursuing in vitro affinity-matrix based binding studies of $\alpha$ SNAP to SNARE complexes [71, 37]. Besides the fact that such high $\alpha$ SNAP concentrations are probably not physiological, their need is also difficult to reconcile with earlier experiments [72, 73], in which optimal functional reconstitution of $\alpha$ SNAP dependent processes was achieved using much lower amounts of $\alpha$ SNAP $(\sim 0.6 \mu \mathrm{M})$. Since, as mentioned above, some of the regulatory mechanisms might aim at the adaptor-level (namely $\alpha$ SNAP), these might stay hidden in a system requiring unnaturally high amounts of $\alpha$ SNAP for function.

Yet, in order to be able to also assess regulatory mechanisms and factors which do not act in an 'all-or-nothing' but rather a modulatory fashion, the conditions during the experiment should be as close to nature as possible. For this it is e.g. desirable, that none of the proteins involved is required in unreasonably high amounts for optimal function. As a prerequisite to addressing regulatory and mechanistic questions on the level of all proteins involved, including the SNAP-adaptor protein, this in vitro system should thus preferably lead to satisfactory reaction rates with lower amounts of $\alpha \mathrm{SNAP}$ than those reported by Lauer et al. [62].

I hence sought to also understand, why the $\alpha$ SNAP affinity seems to be so low in some assays (and thus conditions), in order to get as close to physiological conditions in my in vitro studies as possible. The succesful optimization of the assays finally made it possible to study the mechanisms of NSF-catalyzed SNARE-dissociation on a molecular basis.

\subsection{Part 1 - Characterization of the SNARE Re- action Using Fluorescence Spectroscopy}

The two main approaches used to characterize disassembly in this study, are FRET spectroscopy and fluorescence anisotropy. These fluorescence based read-outs depend on different physical properties. FRET spectroscopy exploits the fact that the amount of FRET (fluorescence resonance energy transfer) is proportional to the inverse power of six of the distance between two fluorophores. If two FRETcompatible fluorophores are attached to different proteins, the interaction of these 
proteins can be seen in the fluorescence spectra of the fluorophores (as detailed in section 4.1.3).

The fluorescence anisotropy varies with the rotational motion of the fluorescence scattering of fluorophores in solution as detailed in section 4.1.3. This property may often be correlated to the size of the protein carrying the fluorophore, and conclusively serve as an indicator of protein binding.

To introduce the fluorescence into the system, we used small maleimide-conjugated fluorescent dyes, which specifically react with cysteine residues to form covalent bondages. An advantage of using these small fluorophores over the use of GFPanaloga is their small size and the possibility to specifically couple them to any cysteine in the molecule under study (provided that the mutation of the remaining cysteines does not impair the molecules' functionality). The spectral properties are therefore better defined and thus enable more defined distance measurements.

Disassembly using FRET spectroscopy. In short, for FRET spectroscopy the three neuronal SNAREs, two of them labelled, were pre-incubated to allow for FRET-complex formation. Subsequent addition of $\alpha$ SNAP and NSF under disassembly conditions then initiated the reaction (see figure 4.2). Several FRET pairs known to be suited for visualization of assembly were tested for their ability to also monitor disassembly. Of course, one would expect the same residues to get close during assembly which are taken apart again during disassembly and conclusively the FRET changes of these reaction should be the reverse of each other. Nevertheless, the labelling or the cysteine-mutations of certain residues might inhibit the disassembly reaction, which had to be ruled out first.

Doing this, two FRET pairs (Sb ${ }^{\text {cys } 28} / \mathrm{SNAP} 25^{\text {cys } 130}$ and $\mathrm{Sb}^{\text {cys } 61} / \mathrm{H} 3^{\text {cys } 225}$ ) were soon found to be suited to monitor disassembly of ternary complexes (figure 4.2 and figure 4.13). It should be pointed out at this stage, that the $\mathrm{Sb}^{\text {cys61OG }} / \mathrm{H}^{\text {cys } 225 T R} / \mathrm{SNAP} 25$ complex turned out to have strong disassembly defects in combination with a range of additional mutations (figure 4.14), which like the $\mathrm{Sb}^{c y s 61} / \mathrm{H}^{c y s 225}$ FRET pair did not noticably inhibit disassembly on their own. Besides the putatively interesting mechanistic implications of these findings, which I will touch on again in the next section, they should also be seen as a reminder of how important it is to have several read-outs (e.g. different label positions) leading to identical results before any findings should be interpreted.

Disassembly and $\alpha$ SNAP binding using fluorescence anisotropy. Similarly, different labelling positions were tested for changes of fluorescence anisotropy during disassembly (figure 4.3). Again several labelling positions were observed to give rise to significant signal changes after addition of NSF, $\alpha \mathrm{SNAP}$ and $\mathrm{Mg}^{2+} / \mathrm{ATP}$.

Basic characteristics of the disassembly reaction. The fluorescence changes indicating SNARE disassembly were shown to be NSF-, $\alpha$ SNAP-, $\mathrm{Mg}^{2+}$ - and ATPdependent in both assays, making it very likely that they in fact represent ongoing 
disassembly. Neither the mutant $\alpha \mathrm{SNAP}^{L 294 A}$ nor a hydrolysis-mutant of NSF, $\mathrm{NSF}^{E 329 Q}$ (data not shown), were able to promote disassembly. Furthermore, an instantaneous block of the reaction could be provoked by addition of AlF3, a compound known to freeze AAA ATPases in a transition state.

In line with reports mentioning a salt-sensitivity of the $\alpha$ SNAP/SNARE-complex interaction $[28,71,67]$, kinetics slowed down extremely in presence of as little as $150 \mathrm{mM} \mathrm{NaCl}$ (data not shown). A salt combination of $120 \mathrm{mM} \mathrm{KGlu}$ and $20 \mathrm{mM}$ KAc, which better mimicks physiological conditions, however did not negatively influence disassembly, which is why I used this saltcomposition for all experiments shown in the thesis.

Detection of $\alpha$ SNAP binding via fluorescence anisotropy. In addition to disassembly, some of the labelling positions can also be used to monitor $\alpha$ SNAP binding to the SNARE complex via fluorescence anisotropy. This was demonstrated for the SNARE complex labelled at $\mathrm{Syb}^{\text {cys } 28}$, where $\alpha$ SNAP binding leads to a sharp increase of anisotropy (section 4.1.5).

The $\alpha \mathrm{SNAP}$ mutant $\alpha \mathrm{SNAP}^{L 294 A}$, which has been reported to bind, but not to disassemble complexes, led to the same anisotropy increase upon addition to the fluorescent complex as wildtype $\alpha$ SNAP. But in contrast to wildtype $\alpha$ SNAP, this mutant did not induce the anisotropy decrease representing disassembly in this method, even when NSF, $\mathrm{Mg}^{2+}$ and ATP were present. These are further evidences for the notion that the rise of anisotropy upon $\alpha \mathrm{SNAP}$-addition actually represents binding, whereas the decrease upon addition in presence of magnesium, ATP and NSF indicates the disassembly.

Quality control of NSF. As mentioned above, the fluorescence read-outs were employed to optimize NSF purification procedures until reproducibly active NSF could be purified. NSF activity was monitored at various stages and different purification conditions, in order to get a better understanding of how NSF may be expressed and purified in a reproducibly active manner. The optimal procedure to produce recombinant NSF was found to be Ni/NTA- followed by gel-filtration chromatography as explained in section 4.1.1 in more detail. NSF purified according to these methods was found to disassemble $\sim 15(+/-2)$ SNARE-complexes per min per $\mu \mathrm{gNSF}$ under conditions in which $\alpha$ SNAP is saturating as calculated by the half times of the reaction. This is similar to the published activity found in a fluorescence based in vitro assay using SNAREs fused to GFP-analoga as substrates. [62, 67]

ATP, NSF and $\alpha$ SNAP dependence. The ATP requirements for disassembly were shown to be in the range of $75 \mu \mathrm{M}$ (figure 4.5). This finding is in line with Matveeva et al. [74], who found that one NSF monomer contains two distinct ATP binding sites with a 100-fold difference in affinity. According to that study, the low affinity binding site exhibits a $\mathrm{K}_{d}$ of $40 \mathrm{nM}$ and is not sufficient to stimulate ATPase activity, whereas the high affinity site with a $\mathrm{K}_{d}$ of $15-20 \mu \mathrm{M}$ stimulates ATP hydrolysis. 
NSF activity was constant when employed in a range of 1nM to 16nM (figure 4.4), which is in agreement with data published with the fluorescence based assay of Lauer et al. [62], where $1 \mathrm{nM}$ to $10 \mathrm{nM}$ were reportet to result in disassembly in a linear range.

As mentioned above, the $\alpha$ SNAP requirements for saturation of SNARE complex binding vary between different sources of literature, many of which report surprisingly low $\alpha$ SNAP affinities. I thus also determined the amounts of $\alpha$ SNAP needed for disassembly in the FRET and the anisotropy read-out and quantified $\alpha$ SNAP binding to SNARE complexes using anisotropy.

It soon became evident that in these assays the $\alpha$ SNAP efficiency is very low as well: More than a seven fold excess of $\alpha$ SNAP did not saturate the kinetics of disassembly (figure 4.6). This may even be an underestimate, because at higher $\alpha$ SNAP concentrations the disassembly speeds reach the time resolution limit of the assay. This is astonishing if one considers that only three $\alpha$ SNAPs are supposed to bind one complex according to previous findings. Later, using lower amounts of NSF and thereby slowing down reaction kinetics, I could demonstrate that even $1.1 \mu \mathrm{M}$ of $\alpha$ SNAP are still limiting when disassembling $\sim 100 \mathrm{nM}$ SNARE complex. The titration experiment shown in section 4.16 indicated that more than $17 \alpha$ SNAP molecules per SNARE complex are needed to saturate binding, when 70nM of SNARE complex are used.

Having observed this low $\alpha$ SNAP efficiency in the fluorescence assays, the question came up, whether there was a problem with the recombinant $\alpha$ SNAP used for the experiments.

On the one hand, as indicated above, the high $\alpha$ SNAP requirements in my assays are in line with Lauer et al.[62], who determined the EC50 for $\alpha$ SNAP binding to SNARE-complexes to be $5 \mu \mathrm{M}$ in their hands, at a complex concentration of only 100nM. Such apparently low $\alpha$ SNAP-affinities have also been observed by other groups persueing in vitro affinity-matrix based binding studies of $\alpha$ SNAP to SNARE complexes.[71, 37].

On the other hand, besides the fact that the high $\alpha$ SNAP concentrations needed to saturate SNARE complex binding are probably not physiological, their need is also difficult to reconcile with earlier experiments [72, 73], in which optimal functional reconstitution of $\alpha \mathrm{SNAP}$-dependent processes was achieved using much lower amounts of $\alpha$ SNAP (less than $\sim 0.6 \mu \mathrm{M}$ ). Furthermore, according to a semiquantitative Western blot performed in our lab, the $\alpha$ SNAP concentration in the cell was estimated to be much lower (T. Lang, personal communication).

It was important to address this question, because excessive needs of one component for optimal function might also point to an imperfect interaction of this component under the conditions used. If this were to be the case for $\alpha \mathrm{SNAP}$, it would not be possible to study the reaction in a meaningful manner, because the reduced binding or the excess amounts of $\alpha$ SNAPs might shift equilibria of the remaining interaction parameters. This might lead to a scenario in which other bottle-necks of the reaction as well as regulatory mechanisms might be overlooked, especially if these act on the adaptor level. 


\subsubsection{The Membrane is a SNAP Receptor}

Possible reasons for low $\alpha$ SNAP efficiency In principle, the low $\alpha$ SNAP efficiency could be inherent to the recombinant $\alpha$ SNAP, possibly due to degradation of only a few residues which would not be visible on a gel or posttranslational modifications present in vivo but absent when expressed in E.coli. Degradation might for instance produce $\alpha$ SNAP which is no longer able to bind SNARE complexes. Consequently, only a fraction of the presumably high amount of $\alpha$ SNAP used in the experiments would be active. Alternatively, the low efficiency might be caused by non-optimal conditions in the fluorescence assays.

The recombinant $\alpha$ SNAP binds SNARE complexes in a 3:1 stoichiometry.

As shown in section 4.3.1, I also investigated $\alpha$ SNAP binding to the SNARE complex using isothermal titration calorimetry. The resulting enthalpies however were very low $(\sim 4 \mathrm{kcal} /$ mole of injectant), making the accurate determination of released energies and consequently of the affinity impossible. The stoichiometry of the SNARE complex could nevertheless be determined to be three $\alpha$ SNAPs per complex in ITC experiments. Notably, this is exactly what is expected and hence allows us to conclude that the majority of the recombinant $\alpha$ SNAP is able to bind SNARE complex, excluding the possibility that degradation is the reason for the low potency. The main difference between the ITC conditions and those during the fluorescence assays being the much higher protein concentrations during ITC measurements, two possible explanations remained: Either does the recombinant $\alpha$ SNAP have a reduced affinity as opposed to endogenous, or a factor important for binding is missing in the fluorescence assay. Notwithstanding the low enthalpy values of the reaction, I still tried to fit the reaction in order to roughly estimate the $\alpha$ SNAP affinity. The thermodynamic data could not be properly fitted using a binding model, which assumes that $\alpha$ SNAP has the same affinity to all binding sites on the SNARE complex. Using a three-site sequential fit however, the curve could nicely be fitted (see figure 4.17). The affinities resulting from this fit were 10nM for the first, $50 \mathrm{nM}$ for the second and $380 \mathrm{nM}$ for the third binding site. Nevertheless, keeping in mind the low enthalpies and also considering that only a low number of data points per binding site were recorded, the affinities of $\alpha$ SNAP to the SNARE complex determined might not be very reliable.

The recombinant $\alpha$ SNAP has a high SNARE-affinity on membrane sheets.

To investigate the efficacy of the recombinant $\alpha$ SNAP under the more physiological conditions of a different system, we performed disassembly measurements using the membrane sheet assay introduced in section 4.3.2.

Surprisingly, in the membrane sheet assay $\sim 100 \mathrm{nM}$ of $\alpha$ SNAP were sufficient to mediate disassembly equally well as $2 \mu \mathrm{M}$, indicating that the $\alpha$ SNAP concentration of 100nM saturates the assay. Of course the amount of SNARE target in these assays is not known, not permitting a conclusion about the stoichiometry of $\alpha$ SNAP and SNARE complexes in this assay. Nevertheless, together with the ITC measurements 
which indicated a molar ratio of $3: 1$, these findings strongly suggest that a factor missing in the FRET and anisotropy experiments but present on membrane sheets is responsible for the higher $\alpha \mathrm{SNAP}$-affinity.

The $\alpha$ SNAP efficiency is enhanced when disassembling liposomal complexes. The most obvious difference between the in vitro fluorescence spectroscopy and the membrane-sheet assay was the absence or presence of lipids, respectively. Since the disassembly reaction in the cell is most likely supposed to occur only after fusion has completed and all three SNAREs are located on one membrane, it is of course tempting to speculate that the membrane is of some importance during the disassembly reaction. One possible way of communication between the disassembly machinery and the membrane would be an interaction of $\alpha \mathrm{SNAP}$ with the membrane in addition to its interaction with the SNARE-complex, which consequently might increase $\alpha$ SNAP/SNARE affinity. Such a scenario is especially feasible, since $\alpha \mathrm{SNAP}$ is an amphiphilic protein which has been reported to bind to plastic-surfaces [51]. Furthermore, $\alpha$ SNAP was shown to bind lipids in a SNARE-independent manner [50].

To test the hypothesis that $\alpha$ SNAP might be more efficient in the presence of membranes, FRET-spectroscopy and anisotropy measurements were carried out using SNARE complex as a target which was incorporated into liposomes prior to the experiment. Indeed, the $\alpha$ SNAP efficacy was found to be increased by a factor of $\sim 10$ to 20 compared to its efficiency in solution (figure 4.22). As little as $45 \mathrm{nM}$ of $\alpha$ SNAP mediated disassembly of $\sim 50 \mathrm{nM}$ liposomal SNARE complex and 120nM were sufficient to saturate the assay (see figure 4.21). The reasons for the improved $\alpha \mathrm{SNAP}$ efficacy in the presence of liposomes were not known at this stage.

NSF activity is not enhanced on liposomes. To investigate, whether the membrane boost is only due to a higher $\alpha$ SNAP affinity or whether the incorporation of complexes into liposomes also influences NSF activity, NSF-action on liposomal and soluble SNARE complexes was compared at $\alpha$ SNAP concentrations promoting optimal disassembly, respectively. Doing so, NSF proved to be as effective on liposomes as in solution, indicating that its activity or SNARE/SNAP affinity are not influenced by membrane incorporation of the SNARE targets. Furthermore, the same amount of an antibody against the N-terminal domain of NSF was needed to block NSF activity on liposomes as in solution, further strengthening the conclusion.

Neither of the SNARE transmembrane domains provides the additional $\alpha$ SNAP binding site. Next, the reasons for the membrane caused potentiation of $\alpha$ SNAP efficiency were elucidated. Alternatively to the lipids now present in the reaction, one of the transmembrane domains used to anchor the SNARE complexes to the liposomes might also be responsible for the improved $\alpha$ SNAP potency. To exclude this possibility, reactions were performed using either only the Synaptobrevin or only the Syntaxin transmembrane domain (figures 4.21 and 4.26). Both showed 
identical $\alpha$ SNAP affinities, demonstrating that neither of the transmembrane domains is responsible for the improved disassembly.

The mere presence of lipids does not suffice. $\quad \gamma$ SNAP and Sec17 have been reported to have highly flexible C-termini, which is why for both of these proteins the very C-terminal residues are not included in the solved crystal structures [30, 29]. One could now argue that this apparent flexibility might hamper function and that the lipids present during the liposome experiments might simply stabilize the $\alpha$ SNAP C-terminus and thereby improve its affinity. The observed potentiation effect would thus not be caused by the membrane anchorage but only result from an optimized stabilization of the recombinant $\alpha$ SNAP. To exclude this possibility, I disassembled SNARE complexes in a mixed micelle solution, where a fraction of SNAREs would be expected to reside on liposomes, whereas the remainder would be in solution. Here, part of the disassembly reaction required only low amounts of $\alpha$ SNAP to proceed, while the remainder only occured at a high $\alpha$ SNAP concentration (see figure 4.29). The fraction of disassembly at low and high $\alpha$ SNAP concentrations correlated with the fractions expected to be liposomal or solubilized, respectively (figure 4.30).

The membrane boost is independent of the lipid composition. Various protein/lipid interactions are known to depend on a specific lipid component. I therefore tested, whether the improved $\alpha$ SNAP function on liposomes is dependent on the lipid composition. To do so, I reduced the heterogeneity of the liposomes, which had been constituted of PC, PE, PI, PS and cholesterol to closely resemble neuronal plasma membrane content in the earlier experiments. Interestingly, disassembly of SNARE complexes on liposomes consisting of only PC and PE showed a similar $\alpha$ SNAP dependence as those on the more heterogeneous liposomes used previously (figure 4.28). Even liposomes consisting of PC only were disassembled at $\alpha$ SNAP concentrations of less than 100nM (data not shown).

The N-terminus of $\alpha$ SNAP mediates lipid binding. These data made it tempting to speculate that a direct interaction between $\alpha \mathrm{SNAP}$ and the membrane lipids is responsible for the increased efficacy on liposomes. If this were true, it should be possible to map the lipid-binding property to a certain region of $\alpha$ SNAP.

Deletion of this region should then abolish the membrane interaction and consequently the observed boost of $\alpha$ SNAP effectivity on membranes. Since no crystal structure of $\alpha$ SNAP has been solved so far, we used the Sec17 crystal structure as a reference to predict the putative $\alpha$ SNAP region responsible for lipid binding (figure 5.1A). The C-terminus of $\alpha \mathrm{SNAP}$ is believed to mediate the interaction with NSF, which is believed to bind to the SNAP/SNARE acceptor-complex from the cytosolic site. According to the current state of evidence, $\alpha$ SNAP and the SNARE complex are thus likely to align in an anti-parallel fashion, which places the $\mathrm{N}$ terminus of $\alpha$ SNAP close to the membrane. Based on the Sec17 structure and 
$\alpha$ SNAP/SNARE-complex interaction studies using various point-mutated $\alpha$ SNAPs, Marz et al. have constructed a model of $\alpha$ SNAP bound to the SNARE complex [67]. An adapted version of this model is shown in figure 5.1 B. Here one can see an armlike structure at the N-terminus of $\alpha \mathrm{SNAP}$ pointing away from the complex, the most far-out region of which (residues 27-32 in $\alpha$ SNAP) includes mostly hydrophobic amino acids. This region corresponds to the loop between helices $\alpha 1$ and $\alpha 2$, designated $\alpha 1^{\prime}$. If this region were the interaction site of $\alpha$ SNAP with the membrane, its deletion should abolish membrane binding and hence the higher $\alpha$ SNAP efficiency on membranes. I therefore cloned an $\alpha$ SNAP-mutant in which I deleted the first 32 residues (designated $\alpha$ delSNAP), in which the critical region would be abrogated. 
A
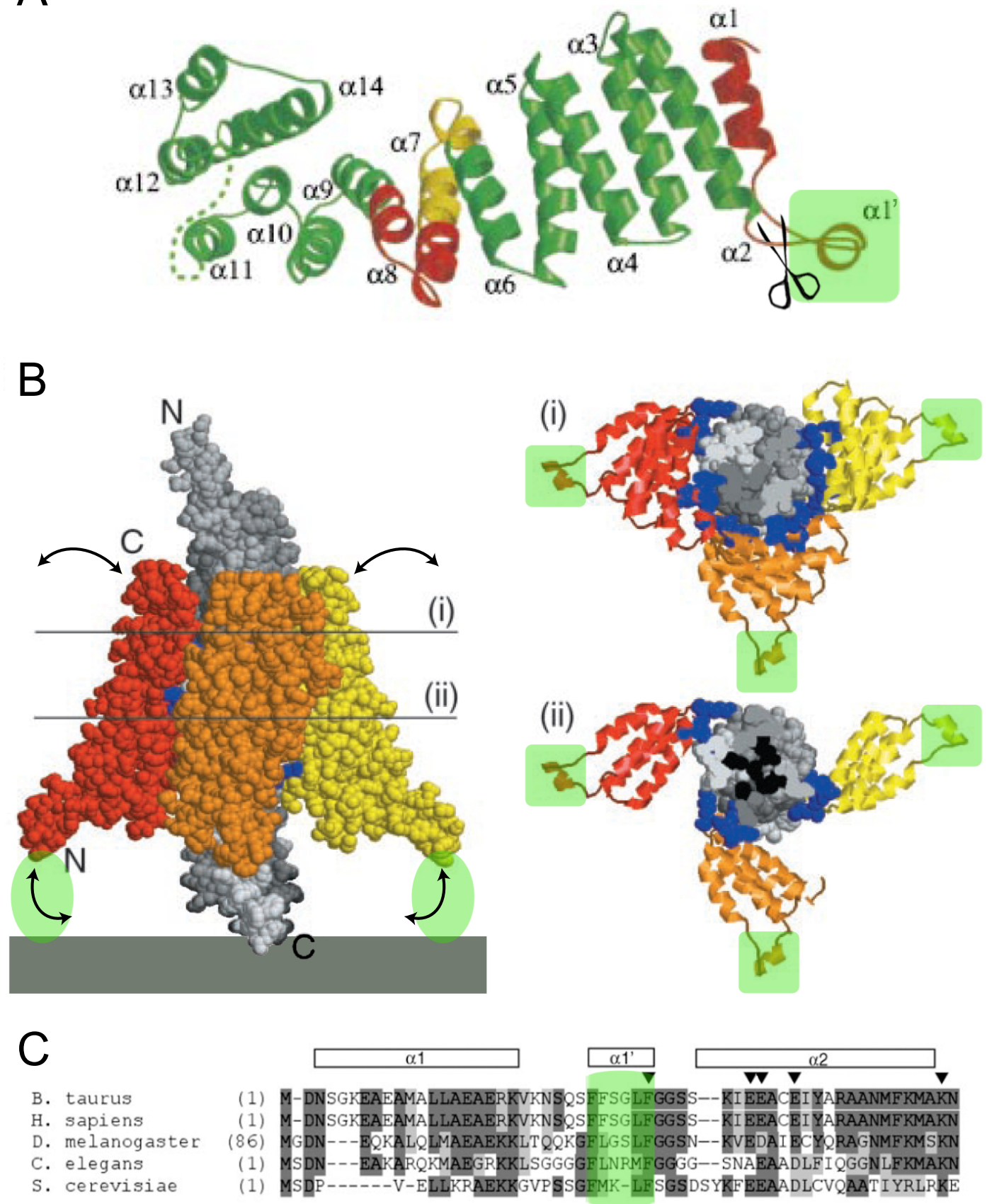

Figure 5.1: The hypothetical lipid binding site of $\alpha$ SNAP. (A) Structure of Sec17. Helix $\alpha 1$ and loop $\alpha 1^{\prime}$ are missing in the $\alpha$ SNAP mutant $\alpha$ delSNAP as indicated by the pair of scissors, (adapted from Rice et al. [30]) (B) Model of $\alpha$ SNAP/SNARE-interaction adapted from [67]. The SNARE complex is depticted in grey and the $\alpha$ SNAPs modelled on top are shown in red, orange and yellow. The $\alpha 1$ ' loop of $\alpha$ SNAP points away from the SNARE complex towards the membrane. This putative $\alpha \mathrm{SNAP} /$ lipid interaction site is highlighted in green. (C) Sequence alignment of several $\alpha$ SNAP homologues. Dark gray shading indicates strong conservation, gray shading conservation. The hydrophobic residues F27 and F32 are highlighted in green and conserved throughout all species. 
First of all it was shown that $\alpha$ delSNAP is able to disassemble, even though at a lower efficiency than $\alpha$ SNAP. This reduced overall efficiency is in line with findings by Hayashi et al [71], who found a $75 \%$ reduction of $\alpha$ SNAP binding to SNARE complexes after deletion of the first 28 amino acids using a GST-binding assay.

As opposed to $\alpha$ SNAP, the kinetics of $\alpha$ delSNAP-mediated disassembly in solution and on liposomes were identical, as we had predicted which is shown in section 4.4.4. As a matter of fact, deleting the first 32 amino acids hence abolished the potentiation of $\alpha \mathrm{SNAP}$ efficiency at the membrane.

Secondly, I directly compared $\alpha$ SNAP and $\alpha$ delSNAP efficiencies on liposomes. Theoretically, it should be predictable at this stage, how much more efficient the wildtype $\alpha$ SNAP should be on liposomes. Now knowing that $\alpha$ delSNAP has an approximately six fold lower efficiency than $\alpha$ SNAP in solution, and from my additional findings (figure 4.22

) that the efficiency of wildtype $\alpha$ SNAP increases by a factor of $10-20$ in the presence of membranes, the efficiency difference on liposomes can be estimated. Accordingly, an efficacy factor in the range of 60-120 (roughly six times ten to twenty), would be expected. As a matter of fact, experiments carried out to confirm this prediction (and hence also confirm the hypothesis that $\alpha$ delSNAP has indeed lost the ability to be boosted by membranes) indicated a 80 -fold higher efficiency for $\alpha$ SNAP.

Taken together, these findings strongly suggest that the N-terminal arm-like structure observable in the crystal structure of $\alpha$ SNAP is essential for a protein/lipidinteraction which mediates the potentiation of complex disassembly at low $\alpha$ SNAP concentrations. An additional $\alpha$ SNAP binding site is hence provided by the membrane lipids, presumably leading to a drastic enhancement of the overall $\alpha$ SNAP affinity to its acceptor site. This might be a mechanism of cooperative binding of the SNARE complex and the membrane, which stabilizes $\alpha$ SNAP at the site of catalysis.

\section{Strategy to further confine the $\alpha$ SNAP residues constituting the lipid} binding site. According to the above-mentioned SNAP/SNARE binding model proposed by Lauer et al. [62], there is a loop close to the N-terminus of $\alpha \mathrm{SNAP}$, which points towards the direction of the membrane. This loop $\alpha 1$ ' contains hydrophobic residues at the positions homologous to F27, F28, L31 and F32 in $\alpha$ SNAP throughout all sequences shown in the alignment in figure $5.1 \mathrm{C}$, two of which are strictly conserved (F27 and F32). Lauer et al. state [62] that the C-terminal globular bundle of $\alpha \mathrm{SNAP}$ was left out of their model in order to allow for the tight binding of $\alpha$ SNAP along the whole SNARE-complex surface. They argue that the globular bundle, if fitted into the model according to the bend observed in the Sec17 structure, would have crashed with the SNARE complex. They justify the removal with the proposal that this C-terminal globular bundle might flexibly bend away upon interaction with the complex. There is however no evidence for this notion apart from the fact that the two structures would collide if the bundle would not have been omitted. Considering the low enthalpy-changes during my ITC measurements 
of $\alpha$ SNAP-binding to the SNARE complex, the interaction between SNAP and the SNARE complex might on the other hand also be weak, so that the two proteins might not be bound as tightly as indicated in the model. Now imagining that the Cterminal bundle of $\alpha$ SNAP were present and would not bend away, one can picture the upper $\alpha$ SNAP portion shown in the model to tilt outwards. This would bring the loop $\alpha 1$ ' even closer to the membrane (which I schematically introduced into the picture) and hence make a interaction of the SNAP and the membrane at this site highly probable (as depicted by arrows). In order to test, whether the lipid binding site of $\alpha \mathrm{SNAP}$ is as actually confined to this loop constituted of 6 residues, an $\alpha$ SNAP mutant could be constructed accordingly and tested as previously described for $\alpha$ delSNAP.

Putative mechanism of the 'membrane boost'. Having shown that an additional binding site between $\alpha$ SNAP and the membrane strongly increases the affinity of $\alpha$ SNAP to the SNARE acceptor, the question still remains, which mechanistic implications the increased $\alpha$ SNAP affinity has for SNARE disassembly. Spontaneously, one can think of two possibilities. For one, the lipids might simply function as a kind of SNAP collector, binding to $\alpha \mathrm{SNAP}$ and thereby increasing its local concentration on the membrane and thus near the complex. The increased local concentration would then support SNAP binding. Alternatively, the SNAP/lipid interaction might actually support the strength of $\alpha$ SNAP binding and maybe even increase the stability of SNAP/SNARE-binding during disassembly. A combination of both scenarios is also conceivable.

The SNAP isoforms $\alpha$ - and $\beta$-SNAP are exchangeable - Even the membrane boost is conserved. Since $\alpha$ - and $\beta$-SNAP have $83 \%$ sequence identity [34], one possibility is that they are simply functionally similar iso- forms. This view is supported by the finding of a single SNAP in Drosophila as well as squid [75] with sequences, which are equally related to those of both mammalian $\alpha$ - and $\beta$-SNAPs.

There have been contradictory findings with respect to $\beta$ SNAP's interchangeability with $\alpha$ SNAP $[33,32,31]$. None of these groups, however, have directly compared $\beta$ SNAP and $\alpha$ SNAP disassembly in a time-resolved experiment. During this thesis, I compared $\alpha$ - and $\beta$ SNAP-mediated SNARE disassembly in solution as well as on liposomes. Due to ongoing degradation problems during purification, the efficiencies of both proteins can not be compared in an absolute manner, but $\beta$ SNAP could be shown to efficiently disassemble neuronal SNARE complexes. In addition I found that the relative potentiation of efficiency is identical from solution to liposomes for both proteins. It can thus be concluded that the membrane boost found for $\alpha$ SNAP is conserved for its isoform $\beta$ SNAP. 


\subsubsection{Summary - Advantages of the Fluorescence-Based Ex- periments over Conventional Disassembly Assays}

The fluorescence assays presented here have major advantages over other published in vitro assays of SNARE disassembly.

Quantitative monitoring of disassembly with high time resolution. First, the efficiency of disassembly can directly be related to the changes during SNAREassembly, providing a direct possibility to quantify the amount of SNARE complex disassembly. Second, signal changes can be recorded online, making the time needed for efficient mixing (approx. 5s, depending on the viscosity of the buffer) the limiting parameter. The reaction can thus be monitored on-line, which additionally allows for the accurate quantification of reaction rates. At the same time, the 'real-time' recordings also make the observation of reversible changes possible. If, for example, there were a point in a reaction under study, at which one of the disassembling factors got limiting and reassembly took over, this would be visible in the spectrum. Adding excess amounts of an unlabelled SNARE of the same type as one of the labelled SNAREs in the complex to the solution can be used to make the reassembly invisible. If Synaptobrevin, which does not bind $\alpha$ SNAP [71] and should thus not interfere with the disassembly reaction, is used for this purpose, different kinetics in the presence or absence of unlabelled Synaptobrevin point towards ongoing reassembly. As opposed to read-outs with a limited time resolution, this approach can therefore be employed to differentiate between defects in disassembly and an enhanced rate of assembly. Additionally, the use of FRET pairs at different positions in the complex also allows to see, whether the SNARE helices or certain parts of these significantly change their position already upon $\alpha$ SNAP or NSF binding. Major structural rearrangements upon docking of the disassembly machinery should hence be observable. However, I did neither observe drastic changes in FRET intensities upon addition of $\alpha$ SNAP and NSF nor when I employed $\mathrm{AlF}_{3}$ which supposedly freezes NSF in a transition state, indicating that (at least with respect to the distance between residue 130 in SNAP25 and residue 28 in Synaptobrevin) no major structural rearrangements take place before the actual dissociation in presence of $\mathrm{Mg}^{2+}$ occurs.

The incorporation of SNAREs into liposomes dramatically increases $\alpha$ SNAP efficiency. The use of liposomes for anchorage of the SNARE complexes is a major improvement of the assay, also with respect to the fluorescence based assay published by Lauer et al. [62]. The additional $\alpha$ SNAP binding site provided by the membrane strongly increases $\alpha$ SNAP affinity to the SNARE-complex compared to the affinity found in membrane-free experimental set-ups. The fluorescence systems established during this thesis hence are the first fully reconstitutedin vitro assays to directly monitor SNARE-complex disassembly, in which the complete high-affinity $\alpha$ SNAP binding site is present. We can thus use stoichiometric amounts of $\alpha$ SNAP for our studies, which is essential to solve questions concerning the $\alpha$ SNAPs/SNARE complex-interactions during disassembly. This is especially interesting, since only 
little is known about the precise mechanism of SNAP/SNARE-interactions during disassembly. Even though three $\alpha$ SNAPs have been determined to bind to one SNARE-complex in purified 20S complexes as determined by quantitative amino acid analysis [61], it is not known whether three $\alpha$ SNAP actually have to be bound for functional disassembly. It would for instance also be possible that one or two $\alpha$ SNAPs suffice to promote disassembly, maybe at a lower speed. All results collected in this study are in line with such a scenario, as will be explained in the next section.

\subsection{Part 2 - Modulating the Reaction}

\subsubsection{Robustness of the Reaction - Bottlenecks and Puta- tive Regulatory Targets}

The second part will focus on different approaches to increase the current understanding of the molecular interactions of NSF, the SNAPs and the SNARE complex during the disassembly reaction. To this end, I applied several strategies to target different components of the reaction, most of which aim for a better understanding of the SNAP/SNARE interface. As mentioned above, one major insight into the molecular details of this interface was the additional $\alpha$ SNAP binding site provided by the membrane lipids, which leads to a dramatically increased SNAP/SNARE affinity.

However, it as yet remains unclear, which parts of the SNARE complex are important for disassembly. Likewise, with respect to the assumed three sites of $\alpha \mathrm{SNAP} / \mathrm{SNARE}$ - and even six sites of NSF/ $\alpha$ SNAP-interaction, it is not known to which degree the machinery is robust in the sense that the specific interruption of single interaction-sites only decreases reaction rates or leads to a complete abolishment of disassembly.

Obviously, one could argue that it should be easy to address this question, especially now that we are able to apply stoichiometric amounts of $\alpha$ SNAP. For instance, one might simply use less than three $\alpha$ SNAPs per complex and quantify the reduction of disassembly. Unfortunately, this is not as easy as it sounds, because so little is known about the characteristics of the SNAP binding site. Neither is it known, whether the SNAP affinity to all sites is equally high or whether they display two or three unequally affine binding sites. Nor do we know, whether there is cooperativity between the different sites, which might lead to the occupation of two or all three sites on one complex before the first site of the next complex will be occupied. When using limiting amounts of $\alpha \mathrm{SNAP}$ we would thus never be sure about whether only part of the complexes is occupied or whether every complex is occupied incompletely. Kinetically, this can not be distinguished either, because due to the enzymatic properties of the reaction, the complexes would simply be disassembled one after another in the first scenario, whereas the second scenario might lead to a reduction of the speeds of the disassembly as such - both would hence result in 
slower total disassembly kinetics.

The ITC measurements performed in section 4.3.1 pointed to a sequential binding (figure 4.17), but as the enthalpies of $\alpha$ SNAP/SNARE complex binding were very low, it is difficult to interpret the measurements and the affinites for the different sites could not be reliably determined and cooperativity thus not be investigated. The anisotropy approaches can not be used to differentiate between different sites of binding either. Even though the anisotropy read-out of $\mathrm{Sb}^{\text {cys28-labelled complexes }}$ serves an an indicator of $\alpha$ SNAP binding, we do not know whether the signal increase seen results from the binding of only one $\alpha \mathrm{SNAP}$ in close vicinity to the label position or whether all three SNAPs contribute to the signal. The collective term of 'the $\alpha$ SNAP affinity' used so far is hence an average property of the amount of $\alpha$ SNAPs needed for optimal disassembly, which does not differentiate between the distinct $\alpha \mathrm{SNAP} / \mathrm{SNARE}$ interaction sites.

Due to the lack of direct possibilities to address the properties of the distinct $\alpha$ SNAP binding sites, indirect approaches may help. If, for example, certain deletions in the SNARE complex led to inhibited disassembly, this might result from an ablation of certain $\alpha$ SNAP binding sites. It should nevertheless be kept in mind that SNARE deletions leading to inhibited disassembly do not necessarily always result from an interrupted interaction site but may as well directly (e.g. sterically) inhibit the mechanics of the disassembly reaction. Anyways, a better knowledge about such coherences might provide clues about the relation between $\alpha$ SNAP binding sites and SNARE-disassembly.

Hence, NSF and the SNARE-complex were selectively altered in order to see, whether the alterations would affect disassembly. It should be pointed out that the experiments discussed in the following section were performed before the discovery of the $\alpha \mathrm{SNAP} /$ lipid interaction and were thus carried out in solution. Since in these experiments either NSF or the SNARE complex, but not $\alpha$ SNAP were modified, $\alpha$ SNAP was always present in excess during the reactions. Alterations completely abrogating the interaction with $\alpha$ SNAP should lead to inhibited disassembly even in the presence of excess $\alpha$ SNAP. Slight alterations of SNAP/SNARE- or NSF/SNAPbinding on the other hand might be overlooked taking this approach in solution.

\section{Inhibition on the level of NSF - Disturbing the NSF/SNAP interaction?}

First, the interaction interface between NSF and the SNAPs was addressed. Experiments performed with the monoclonal anti-N antibody (type IgG2) generated in the context of this study led to a complete block of disassembly. It is likely that the antibody interferes with NSF binding to $\alpha$ SNAP, which has been reported to depend on the NSF N-terminal domain [22]. Since all $\alpha$ SNAP/NSF connection sites presumably are structurally identical, a structural change in one of the proteins is likely to affect all SNAP/NSF interactions equally, and consequently the complete abolishment of SNARE disassembly is not surprising. It should nevertheless be noted, that the antibody might also inhibit the reaction by different means, e.g. steric hindrance of the NSF molecule during disassembly. The antibody was shown to specifically recognize only NSF of all the proteins present in cytosol, and might 
hence also serve useful as a tool to specifically block disassembly in in vivo studies in the future.

Alterations on the target-level. The minimal SNARE regions required for SNARE disassembly [6] as well as various mutants which may or may not inhibit SNARE disassembly have been described [71, 64, 62, 65]. SNARE assembly has been shown to exhibit an N-terminal nucleation making the N-terminal residues obligatory for efficient assembly. It is possible that for disassembly analogous obligatory residues exist. For example, the conservation of the ' 0 '-layer residues has originally been suggested to be essential for disassembly. This led to the hypothesis, that the '0'-layer might be the area of attack of the disassembly machinery [64, 65]. In the scope of this thesis, I re-investigated some of these findings as well as some additional SNARE complex alterations, exploiting the increased time resolution of the fluorescence assays as compared to the approaches used in the original publications.

As pointed out earlier, due to the asymmetric structure of the SNARE complex, the interaction between the SNAPs and the SNARE-complex target is mediated by at least two, and most likely three, structurally different sites. Losses of interaction at any of these three sites as well as their consequences can thus be addressed independently.

The disassembly reaction tolerates 'single-locus' target alterations. First, I ensured that the complexes containing only the H3 domain of Syntaxin are equally prone to disassembly as full-length Syntaxin, which supports earlier findings from our lab gathered with the gel-based disassembly assay [6]. Knowing this, I used only the $\mathrm{H} 3$ domain for all remaining experiments performed in solution. To elucidate whether certain regions of the SNARE complex are essential for disassembly, I next investigated various SNARE complex mutants.

Amongst these were the Synaptobrevin '0'layer mutant $\left(\mathrm{Sb}^{R 56 P}\right)$ as well as the Syntaxin '0'layer mutant which have both led to controversial results between different approaches in the literature. In my hands, both mutants did not inhibit disassembly in the fluorescence assays as demonstrated in figure 4.9, which is in line with the findings of Lauer et al. [62] that were published during this work. Both mutants have been reported to inhibit disassembly before [64, 65], but these findings might also stem from an enhanced re-assembly capacity of the mutant SNAREs, which would not be differentiated in their gel-based disassembly assays. Alternatively, a slightly reduced $\alpha$ SNAP affinity to the mutated complexes might be the reason for the disassembly defects observed by Scales et al. and Martinz-Arca et $a l$., which might not be apparent in my fluorescence measurements performed in the presence of excess $\alpha$ SNAP. Indeed, a slightly reduced affinity of $\alpha$ SNAP to the Syntaxin $^{R 226 Q}$ containing complex was reported by Lauer et al. [62].

Furthermore, C-terminal deletions of SNAP25 as well as point mutations exchanging the two amino acids which SNAP25 contributes to the '5'-layer of the neuronal SNARE-complex (SNAP25 $5^{M 71 A, I 192 A}$ ) did not inhibit SNARE-complex disassembly as shown in figure 4.10. Likewise, a C-terminal Synaptobrevin deletion (Sb1- 
70) did not impair disassembly at all, whereas a C-terminal Sx deletion (Sx183-240) led to a slight inhibition (figure 4.11). The Sx mutant however did not reduce total amounts of disassembly but only slightly impaired the reaction rate at the later stages. Even when both, Sb and H3, were concertedly deleted, the inhibitory effect did not increase.

In summary, none of these mutants lead to efficient inhibition of SNARE disassembly. These findings lead to the conclusion, that the interaction interface between the disassembly machinery and the SNARE complex is not confined to a single site (as e.g. the '0'-layer or the C-terminus) on the SNARE complex but probably covers a larger portion of the SNARE surface. Partial interruption of the interface might then weaken the interactions but not abolish it completely.

Even though it is interesting that the disassembly reaction seems to be robust enough to tolerate these various target alterations, the experiments pursued this far did not lead to any mechanistic insights concerning the putative bottlenecks or protein/protein interactions critical for the performance of disassembly. When searching for efficient ways to disturb disassembly, as an alternative to selectively deleting target-regions one can add factors which might impede either target/adapterrecognition or hinder the reaction sterically. Good candidates for proteins whose attachment might influence function are antibodies. To this end, I tested three antiSNARE complex antibodies for their effectiveness to block disassembly. Two of these antibodies (132.1 and 132.2, see figure 4.2.3) indeed partially inhibited disassembly. However, as also observed for the deletion mutants, both of these antibodies did not inhibit disassembly completely. According to the SNAP/SNARE interaction-model proposed by Lauer et al., the $\alpha$ SNAPs cover almost the whole surface of the complex when all three are bound. If this model is correct, it is highly unlikely that none of the many complex alterations or antibodies tested interfered with any of the SNAP/SNARE binding sites. This might indicate that disassembly may be able to proceed, even when less than three $\alpha$ SNAPs are bound. At the same time it is also possible, that the single mutations do not completely abrogate SNAP/SNARE binding at one site but only reduce the interface of binding and thus destabilize the interaction. The two $\alpha$ SNAPs bound to the remaining SNAP/SNARE interfaces might then be sufficient to compensate for certain destabilizations at one site by a mechanism of cooperativity. The only conclusion that can thus be drawn from these experiments is that the disassembly reaction is robust in the sense that deletions at single interaction interfaces can be tolerated.

Interference at two distant sites can result in severe inhibition. To further strengthen the notion that less than three SNAPs may be able to disassemble one SNARE complex, dual target alterations at non-proximal sites were investigated next, aiming at a possible disruption of more than one SNAP/SNARE binding site which might lead to more pronounced disassembly defects.

As a matter of fact, even though none of the mutations or antibodies discussed in the last paragraph led to severe impairment of disassembly, the reaction could ultimately be blocked in several cases, when two of these mutants or antibodies were 
combined. For one, in the presence of both of the two partially inhibitory antibodies, 132.1 and 132.2, the reaction came to a complete halt. Similarly, the combination of the FRET-pair $\mathrm{Sb}^{\text {cys } 61} / \mathrm{H}^{225}$ and any of the C-terminal or 5-layer SNAP mutants abrogated disassembly almost completely. Conclusively, one defect on the target level does not seem to be enough to abrogate disassembly, whereas the combination of two mutations can strongly interfere with disassembly, even for deletions which are completely silent with respect to disassembly when present independently.

\section{Conclusion: The disassembly mechanism might tolerate partial impair-} ment of $\alpha$ SNAP binding. Interestingly, none of the various single-site mutations of the SNARE complex described above severely inhibited SNARE disassembly, most did not even disturb disassembly at all. Even the combination of two C-terminal SNARE deletion mutants (Sb and H3) did not increase the slight inhibition witnessed for $\mathrm{H} 3$ alone. Only when two different loci on the SNARE complex were disturbed, severe inhibition of the reaction could be observed.

It can not be excluded that slight impairments on the level of $\alpha$ SNAP binding, which might lead to less disassembly, are overseen in these experiments, because they were performed using excess amounts of $\alpha$ SNAP. Alterations which strongly interfere with $\alpha$ SNAP binding should be observable, but we would not know if mutations led to a sightly changed $\alpha$ SNAP affinity. The single-site mutants found to function like wildtype here, can hence not be excluded to have slightly changed $\alpha$ SNAP bindingcharacteristics compared to the wildtype complex. To elucidate whether this is the case for several of the single-site mutants and if so, whether this has an impact on the kinetics of disassembly when more physiological amounts of $\alpha$ SNAP are used, one could carry out these experiments again, this time on liposomes which allow for the use of stoichiometric and substoichiometric amounts of $\alpha$ SNAP.

What can nevertheless be seen from these experiments, is that even in the presence of excess amounts of $\alpha$ SNAP, the complex can be disrupted severely enough to abolish disassembly. Strikingly, these severe inhibitions are brought upon only when several mutations are introduced together, the mutations can thus have synergistic effects. Interestingly, only when two different loci on the SNARE complex were disturbed, severe inhibition of the reaction could be observed, whereas for instance two nearby deletions at the C-terminus (shortened Syntaxin and Synaptobrevin) did not show synergistic effects. It is tempting to speculate that the defects result from ablations of $\alpha \mathrm{SNAP} / \mathrm{SNARE}$ complex binding. If one thinks along these lines, the observations suggest that the NSF machinery indeed might be robust enough to function even though one of the interaction sites between substrate and machinery is weakened or blocked. Considering that the antibodies have been mapped to different regions of the complex, it is possible that the two synergistic clones 131.1 and 131.2 interfere with different SNAP/SNARE interaction sites. Similarly, the other structural alterations of the neuronal SNARE complex which also showed synergistic effects, are located such that they might interfere with different SNAP binding sites. This might indicate that even if one site is weakened or blocked, other sites might be able to compensate for the defect and suffice to mediate disassembly. 
Notwithstanding the fascination of this hypothesis, it might as well be, that the SNARE-complex alterations do not interfere with $\alpha$ SNAP binding but rather change the target structure in such a way that the conformational changes required for efficient disassembly can not occur sufficiently. This possibility is easy to picture when thinking of bound antibodies to the SNARE complex, which might lead to steric hindrance. For the defects caused in the $\mathrm{Sb}^{61 O G} \mathrm{H} 3^{225 T R}$-complexes combined with the SNAP25-mutations this possibility is albeit less plausible, because the strong defects occured when an additional deletion as opposed to an attachment was introduced.

To further elucidate the question, whether less than three $\alpha$ SNAPs per SNARE complex can mediate disassembly, I will now switch perspectives, away from the target- to the adapter-level. Whereas the previous experiments were aiming at interrupting SNARE/SNAP interactions via complex-alterations, I now searched for a way to directly hinder $\alpha$ SNAP-binding without the need of destroying the SNARE complex.

\subsubsection{Inhibition on the $\alpha$ SNAP Level}

To specifically inhibit the SNARE/SNAP interaction face via changes on the level of $\alpha$ SNAP, several possibilities exist. Obviously, in analogy to the experiments using altered SNAREs described in the last section, one could delete or mutate specific regions or residues of $\alpha$ SNAP and afterwards look at these mutants' effects on disassembly. This strategy has for instance been employed by Marz et al [67], who mutated various acidic and basic $\alpha$ SNAP residues and could thereby show that the SNARE/SNAP interaction is mediated by mostly ionic interactions. The model constructed hereafter is illustrated in figure $5.1 \mathrm{~B}$. These studies furthermore showed that the SNAP/SNARE interaction involves residues distributed along almost the whole stretch of the nine N-terminal $\alpha$ SNAP helices rather than being restricted to a small region. Even though these are interesting findings, this strategy is not useful when trying to distinguish between the different SNAP/SNARE interaction sites. A mutation in the $\alpha$ SNAPs itself might, but most probably will not, selectively inhibit one specific SNAP/SNARE interaction but would rather affect all three of them. Even if, theoretically, a mutant would interfere with binding at one specific SNARE site only, we would not be able to tell from the spectral data. A better strategy to ablate binding of $\alpha$ SNAP at only one or two sites, would therefore be to employ a mixture of $\alpha$ SNAP together with an $\alpha$ SNAP competitor, which competes for only one or two of the SNAP binding sites.

Complexins inhibit SNARE disassembly in vitro. One putative candidate for such a protein is Complexin. Complexins are small soluble proteins, which bind as an $\alpha$-helix to the SNARE complex four-helix-bundle in an anti parallel fashion $[39,38,41,76,42]$. Additionally, single molecule studies have recently shown that Complexin may bind to and thereby stabilize Syntaxin/SNAP25 acceptor complexes [77]. However, it does not bind the individual SNAREs or the 2:1 SNARE complex 
consisting of two Syntaxins and one SNAP25 as has been shown by isothermal titration calorimetry [41]. It is therefore highly probable that their functional target in the cell is the assembled SNARE complex and/or the Syntaxin/SNAP25 1:1 acceptor complex, but the precise role of Complexins is not understood up to date.

Complexins have originally been reported to displace $\alpha$ SNAP from the SNARE complex when added in excessive amounts, leading to speculations that they might have inhibitory properties with respect to SNARE disassembly [39]. Notwithstanding these findings, the gel-based in vitro assay monitoring SNARE disassembly introduced in section 4.1.2, did not show any negative influence of Complexin1 or Complexin2 on SNARE-complex disassembly [40]. However, due to a time resolution in the range of several minutes, these experiments are preferably suited for comparing 'all-or-nothing' disassembly defects rather than minor kinetic differences which could hence not be ruled out.

Recently, three different studies resulted in findings which all led to an interpretation according to which Complexin might act as a so-called fusion clamp, binding the SNARE complex in a partially zippered state prior to complete fusion and thereby 'clamping' it until the signal for the final fusion step is received $[43,44,45]$. If this were so, Complexin might preferentially act on 'trans' rather than 'cis' complexes and might conclusively act at a different stage in the SNARE cycle than $\alpha$ SNAP. Hence, even though Complexin seems to be able to replace $\alpha$ SNAP from SNARE complexes [39], a resulting inhibition of the disassembly in in vitro reaction would not necessarily imply that the two competing proteins ever meet in the cell and it would hence be difficult to draw physiological conclusions.

Anyhow, as pointed out above, in vitro experiments using Complexin might serve as a tool to address the question, whether less than three $\alpha$ SNAPs suffice to mediate efficient SNARE disassembly. I therefore tested SNARE disassembly in the presence of Complexin1 (see figure 4.39) using the fluorescence assays and found that the rate of disassembly indeed decreased. The inhibition dramatically increased under conditions in which $\alpha$ SNAP is limiting (figure $4.39 \mathrm{~B}$ and 4.40 ), and the ratio of $\alpha$ SNAP to Complexin was crucial for the degree of inhibition. These findings strongly suggest that the disassembly defect witnessed in presence of Complexin indeed results from a direct competition with $\alpha$ SNAP.

Evidence for SNARE disassembly mediated by less than three $\alpha$ SNAPs?

Conveniently, the structure of Complexin bound to the neuronal SNARE complex has been solved [42], enabling for speculations about the nature of the possible $\alpha$ SNAP/Complexin1 competition with respect to the different binding sites. The Complexin/SNARE-complex binding site overlaps with only one of the three hypothesized SNAP binding sites, according to the SNAP/SNARE model by Marz et al. discussed above. This of course does not necessarily mean that the other two SNAP binding sites are unaffected by Complexin binding, because mechanisms of cooperativity might complicate the picture of the molecular interactions at the SNAP binding sites.

Complexin was found to bind the SNARE complex with a very high affinity in 
solution (less than 10nM) [41], suggesting that it should be dominant over $\alpha$ SNAP with respect to SNARE complex binding at the one overlapping binding site in solution. On membranes however, the affinity of Complexin to SNARE complexes might be much lower $(\sim 680 \mathrm{nM})$, as has been reported by Bowen et al [78] who performed single molecule FRET measurements on deposited lipid bilayers.

The fact that Complexin does not abolish disassembly completely, even though its SNARE binding site overlaps with one of the $\alpha$ SNAP's, may be seen as a further evidence for the considerations that less than three $\alpha$ SNAPs per complex can mediate disassembly. On the other hand, the partial disassembly could also be due to Complexin binding to only a part of the complexes, the remainder of the complexes carrying three $\alpha$ SNAPs and thus being dissociated. Along these lines, a similar amount of inhibition would relate to a similar ratio of Complexin-containing to Complexin-free SNARE complexes. If the reasons for an intermediate inhibition were due to an equilibrium of $\alpha \mathrm{SNAP}$ and Complexin at one site, one should hence be able to shift the equilibrium towards Complexin-bound complexes by increasing the Complexin concentration.

One general observation I made during the experiments however was that the degree of disassembly does not seem to be continuous, but rather seems to be restricted to discrete levels. For example, as shown in figure 4.41, even a five fold increase of Complexin ( $75 \mathrm{nM}$ to $370 \mathrm{nM})$ at a constant $\alpha$ SNAP concentration $(45 \mathrm{nM})$ only slightly increases the degree of inhibition. Both of these different Complexin concentrations led to an intermediate block of SNARE disassembly. Likewise, lowering the $\alpha \mathrm{SNAP}$ concentration to $22,5 \mathrm{nM}$ at $75 \mathrm{nM}$ Complexin results in identical speeds as $45,5 \mathrm{nM} \alpha \mathrm{SNAP}$ and $370 \mathrm{nM}$ of Complexin.

A stepwise dependence of disassembly speeds in presence of varying amounts of $\alpha$ SNAP and Complexin would match nicely with the idea, that discrete rates of disassembly might reflect a different number of $\alpha$ SNAPs bound. Depending on how close the affinities between the sites are to each other, the transitions might be more or less discrete at different occupation levels. Discrete levels however are difficult to reconcile with a view in which the reaction rate only relates to the portion of complexes occupied by Complexin.

These considerations might hence suggest that $\alpha$ SNAP is still able to mediate disassembly even though one SNAP/SNARE interaction site is blocked by Complexin. On the other hand, as already discussed for the SNARE deletion experiments, the site of Complexin binding might, even though it overlaps with one of the proposed $\alpha$ SNAP binding sites, not abrogate SNAP binding to this site completely but only partially. The partial binding of SNAP to the SNARE complex might still suffice for disassembly, maybe through a stabilizing effect mediated by the remaining SNAPs.

Since we do not know whether $\alpha$ SNAP binding to the different sites is cooperative, we do not know either, whether the physical ablation of one SNAP/SNARE interaction mechanistically influences the other SNAP/SNARE interaction. It is for instance conceivable that the affinities to the remaining $\alpha$ SNAP/SNARE sites increases after the first $\alpha \mathrm{SNAP}$ has bound. If now this first site were occupied by Complexin, the two additional sites might display a lower affinity. The fact that 
the impediment of disassembly is only partial however indicates that at least one $\alpha \mathrm{SNAP}$ is still able to bind to the complex even when Complexin is present.

Interestingly, when very low amounts of $\alpha$ SNAP $(22,5 \mathrm{nM})$ are used, which in the absence of Complexin promote only slow disassembly, Complexin can even abolish disassembly completely 4.42 . This may either indicate that the affinities as a matter of fact differ between the three SNAP binding sites, the Complexin binding site at the same time being the one displaying the highest affinity for $\alpha$ SNAP. If then this site were occupied by Complexin, $\alpha$ SNAP might not be able to bind the complex at all, when present only at concentrations sufficient for binding to the first binding site.

In summary, it is hence possible that the reduced rate of disassembly caused by Complexin reflects the kinetics of a reaction where only one or two as opposed to the possible three SNAPs are bound. This would, in accord with the SNARE complex mutation experiments discussed earlier, point to a possible existence of finetuning mechanisms of SNARE-complex disassembly, which act via a modulation of the number of $\alpha$ SNAPs bound per complex. Such a mechanism, which allows for the independent action of one or two SNAPs rather than depending on a concerted action of all three SNAP proteins, would resemble a feature recently discovered for another triple A ATPase called ClpX [27]. Here the authors could show that the single subunits of one ClpX-hexamer were capable to exert their catalytic function independently from each other, whereas a concerted action of several subunits apparently increased the enzymatic efficiency in a proportional dependence. If NSF also were to act in such an 'asymmetric' fashion, there might be a correspondent dependence of NSF activity on the number of SNAPs bound per SNARE complex.

However, all the inhibitory effects described might as well be explained by an influence of Complexin on $\alpha \mathrm{SNAP}$ activity, which is not caused by the mere occupation of an $\alpha$ SNAP interaction site, which can not be excluded at this stage. In order to further address the model of potential discrete levels of $\alpha$ SNAP modulation depending on the amount of functional SNARE/SNAP interfaces, further Complexinand $\alpha \mathrm{SNAP}$-titration experiments are necessary.

A modulatory rather than dominant negative function of Complexin as suggested by the experiments shown, might also help to abate some of the confusion amongst Complexin investigators. For a long time, seemingly contradictory outcomes of knock-out experiments and over-expression studies in various animals and cell-types have complicated the determination of Complexin function, leaving a range of proposed models in the field up to now. Most of these studies report a reduction of exocytosis in presence of excess Complexin, which would be in line with an negative impact on fusion. However, at the same time the absence of Complexin appears to result in impaired exocytosis as well. These apparently contradictory results could in principle also be explained by a fine-tuning capability which for instance might depend on a physiological Complexin concentration. If this is the case, too little as well as too much Complexin would harm the balanced fusion process and might lead to similar phenotypes. 
$\alpha$ SNAP has a selective advantage over Complexin on liposomes as opposed to solution. Recently, three different studies resulted in findings which led to a similar interpretation, according to which Complexin might act as a socalled fusion clamp, binding the SNARE complex in a partially zippered state prior to complete fusion and thereby 'clamping' it until the signal for the final fusion step is received $[43,44,45]$. Even though this scenario primarily envisions Complexin to function as an inhibitor of the last step of SNARE assembly, it does not exclude the possibility that the 'Complexin clamp' serves as a protection against unwanted disassembly at the same time. If for instance, SNARE-complex assembly has almost completed, $\alpha$ SNAP might already recognize its binding sites and induce NSF-mediated disassembly of 'trans' complexes. At this stage, Complexin might have to efficiently compete with $\alpha$ SNAP. After fusion however, it would be logical if $\alpha \mathrm{SNAP}$ were able to overcome the Complexin mediated inhibition of disassembly in order to allow for fast recycling of cis SNARE complexes.

In line with the observation that the $\alpha$ SNAP affinity is strongly enhanced on membranes compared to solution, whereas complexin apparently displays a higher affinity to SNARE complexes in solution than on membranes [41, 79], the ratio of Complexin to $\alpha$ SNAP needed to efficiently block SNARE disassembly in the fluorescence assays, differs significantly between experiments pursued in solution and on liposomes. In solution, sub-molar amounts of Complexin compared to $\alpha$ SNAP already impair disassembly. On liposomes, the amount of Complexin needed to inhibit disassembly is in the same range as in solution, whereas the power of $\alpha$ SNAP increases by a factor of $\sim$ twenty due to the membrane potentiation. As opposed to $\alpha$ SNAP, complexin function hence does not seem to be boosted by the membrane anchorage SNARE complex.

Complexin vs. $\alpha$ SNAP at physiological $\alpha$ SNAP concentrations. According to my knowledge, there are no published data about the physiological concentration of Complexin 1 and $\alpha$ SNAP in the cell, or more precisely, at the sites of SNARE action. According to semi-quantitative Western blotting carried out in our lab, the cellular $\alpha$ SNAP concentration was determined to be in the range of $\sim 20-50 \mathrm{nM}$ (T. Lang, personal communication). This of course does not imply the $\alpha$ SNAP concentration at the membrane is identical. Yet it is likely that the low amounts ( $\sim 100 \mathrm{nM}$ or less), seen to promote optimal disassembly on membranes but not in solution, are closer to physiological concentrations than the high amounts required for optimal disassembly in solution $(\sim 1 \mu \mathrm{M}$ or more). At $100 \mathrm{nM} \alpha \mathrm{SNAP}$, as much as 370nM Complexin did not suffice to block disassembly on liposomes, whereas the same amount of Complexin even impairs disassembly mediated by more than $1 \mu \mathrm{M} \alpha \mathrm{SNAP}$ in solution. One might of course argue that the characteristics of disassembly in solution are anyways of no physiological significance. Still, what we do not know so far, is whether the membrane boost observed for $\alpha$ SNAP is only applicable when the complex resides in 'cis' and all three $\alpha$ SNAPs can thus attach to the same membrane, or whether the boost would also occur with regard to binding of complexes docked in a 'trans' state. If the anchorage of all SNAREs to 
the same membrane were a prerequisite for the high $\alpha$ SNAP efficiency, Complexin would be dominant over $\alpha$ SNAP before, whereas in turn $\alpha$ SNAP would be superior after fusion. However, since we do neither know the physiological concentration of Complexin nor whether these two proteins ever meet during the SNARE cycle, this at the moment is mere speculation and requires further investigation.

Summary of Complexin function in SNARE disassembly. Taken together, the data show that Complexin inhibits SNARE disassembly and the amount of inhibition is dependent on the $\alpha \mathrm{SNAP}$ concentration. Furthermore, the Complexin block is only partial at intermediate $\alpha$ SNAP concentrations but can be complete, when $\alpha$ SNAP is reduced to a minimum. At this point, no definite conclusions can be drawn with regard to whether Complexin binding completely abolishes one or more $\alpha$ SNAP/SNARE interaction sites. Finally, as opposed to $\alpha$ SNAP, Complexin does not show an increased affinity to liposomal 'cis' complexes over ones in solution, leading to an inversion of masteries between Complexin and $\alpha$ SNAP, depending on whether the target complex is on liposomes or in solution. Yet, soluble SNARE complexes do not represent a physiological target, so that a physiological relevance of possible interpretations of this finding can not be concluded.

\subsubsection{Phosphorylation of NSF as a Regulatory Mechanism?}

Another level of regulation might be modifications of the enzyme itself. Several reversible modification mechanisms have been proposed, which might have an impact on NSF activity. For instance, one NSF modification which has been suggested to regulate the disassembly reaction is phosphorylation at residue Y83. According to Huynh et al. [36], the defect is caused by a reduced affinity to $\alpha$ SNAP. To validate the notion that phosphorylation of NSF at this site alters SNARE disassembly, I employed in vitro phosphorylated NSF and a phosphomimetic NSF mutant NSF $\left({ }^{Y 83 E}\right)$ for disassembly in the in vitro assays. Indeed, when I tested the mutant $\mathrm{NSF}^{Y 83 E}$ in the gel based disassembly assay, the mutant seemed to perform less efficiently than the wild type (figure 4.1.2). Since however, as pointed out earlier, the gel experiment is only semi-quantitative and the reasons for a changed outcome are more difficult to assess, such a difference in band intensities does not suffice for mechanistic interpretations.

In this context, I disassembled different complexes, incorporated into the membrane either via the Syntaxin or the Synaptobrevin domain and containing either full length Syntaxin or only the Syntaxin H3 domain, to ensure that no defect only evident for one of these complexes would be overseen (figures 4.45 and 4.44).

$\mathrm{NSF}^{Y 83 E}$ rapidly disassembles on liposomes. Even though there were slight kinetic differences between wild type and $\mathrm{NSF}^{Y 83 E}$ on liposomes, increasing the mutant's concentration by $50 \%$ completely abrogated the differences so that disassembly proceeded with the same kinetics as wild type NSF. A difference as subtle as that is not significant enough to definitely assign a defect to NSF phosphorylated 
at position 83. The fact that such a low increase of the mutant concentration can overcome the differences, might for instance be due to slightly less active enzyme in the mutant's preparation. This for instance might result from reduced stability of the mutant. At this stage it should again be pointed out that NSF is known to be a fragile enzyme and it is difficult to compare the preparations of the wild type and the point mutation quantitatively. It can never be excluded that differences between the enzymes stem from buffering conditions which even though optimized for NSF as described in section 4.1.3, do not mimic the enzymes natural environment. Furthermore, the conditions used in the fluorescence assays were optimized for NSF which does not necessarily imply that they are also the best possible for modified NSF variants, as $\mathrm{NSF}^{Y 83 E}$. It can hence not be excluded that the purification procedures differentially affect the active state of the two enzymes, e.g. by changing the ratio of the hexameric to the monomeric pool and the subtle differences observed do therefore not necessarily represent physiological modulations. These experiments thus do not prove a significant change of disassembly kinetics upon phosphorylation of NSF.

\section{$\mathrm{NSF}^{Y 83 E}$ disassembly might be slightly impaired under certain conditions} in solution. In solution, phosphorylated NSF once more disassembled slower and less efficiently than wildtype when used at identical concentrations at $1,1 \mu \mathrm{M}$ $\alpha$ SNAP (figure 4.46). As opposed to the liposomal findings however, this deficiency could not be overcome by more phosphorylated NSF. Instead, the defect was almost completely abrogated by increasing the $\alpha \mathrm{SNAP}$ concentration from $1,1 \mu \mathrm{M}$ to $2,2 \mu \mathrm{M}$. In the gel-based disassembly assay, NSFY $83 E$ also performed less efficiently than wildtype NSF. I next examined, whether this is due to a reduced $\alpha \mathrm{SNAP}$ affinity of $\mathrm{NSF}^{Y 83 E}$ in solution, as has been suggested by Huynh et al. [36]. To do so, I performed disassembly experiments using $\mathrm{NSF}$ wt and $\mathrm{NSF}^{Y 83 E}$, respectively, at very low $\alpha$ SNAP concentrations. However, the kinetics of NSF and $\mathrm{NSF}^{Y 83 E}$-disassembly in these experiments were similar (data not shown). Similarly, the use of very low enzyme concentrations (NSF or phosporylated NSF, respectively) led to comparable kinetics between wildtype and mutant. A general lower $\alpha$ SNAP affinity can thus be excluded, because this would have been evident under conditions in which $\alpha$ SNAP is limiting.

How may this be interpreted? If there is a significant difference between the two mutants, it thus only seems to come to the fore in a specific range of $\alpha$ SNAP concentrations. If one keeps in mind that the disassembly reaction using wildtype NSF is quite robust, phosphorylated NSF might for instance be more fragile with respect to alterations or inhibitory factors at the SNARE- or $\alpha$ SNAP interaction site. One could speculate that the level of NSF-activity induced by $\alpha$ SNAP might depend on the number or tightness of the $\alpha$ SNAP molecules bound to the complex and this induction pattern might for instance be different after phosphorylation. It is for instance possible that complexes occupied by one or three $\alpha$ SNAPs stimulate phosphorylated NSF as efficiently as wildtype NSF, whereas complexes occupied by 
two $\alpha$ SNAPs induce the activity of phosphorylated to a smaller extent than that of wildtype NSF. Anyhow, since at this point I have not even been able to prove that wildtype disassembly is indeed possible with less than three $\alpha$ SNAPs, these are mere speculations and further experiments, covering a whole range of $\alpha$ SNAP concentrations, need to be performed in order to finally elucidate the function of NSF phosphorylation. Alternatively to a disassembly defect, kinetic differences of disassembly recordings in solution may always as well be caused by different speeds of reassembly. Instead of the proposed disassembly defect of phosphorylated NSF, it might also be less efficient than NSF in keeping the freed SNAREs from re-assembly or only disassemble up to a state of partial disassembly, from which re-assembly may occur faster. Since high $\alpha$ SNAP levels negatively influence SNARE complex assembly kinetics, the apparently better performance of $\mathrm{NSF}^{Y 83 E}$ in the presence of high amounts of $\alpha$ SNAP might as well be caused by a reduction of re-assembly upon raising the $\alpha$ SNAP concentration. Since all these thoughts are speculative, further studies are essential to gain insights into whether the phosphorylation indeed leads to a mechanistic difference of the mutant or whether the mutant is simply less stable in my hands than the wildtype.

\subsubsection{Conservation of the Reaction Mechanism in Yeast}

The fact that $\alpha \mathrm{SNAP}$ and NSF are ubiquitously expressed in all tissues and mediate the recycling of most (if not all) SNARE proteins involved in intracellular fusion processes points at a strong mechanistic conservation of the different disassembly reactions throughout the cell. Considering that the four-helix-bundle structures of the different SNARE complexes show a high degree of similarity, the overall sequence homology between the different complexes studied thus far is surprisingly low. It is hence possible that the SNAP/SNARE machinery does not care about conservation of specific residues but rather recognizes its target proteins on the basis of their quaternary structure. Several studies have already focused on the question, of whether even between two organisms as distant as yeast and mammals the degree of conservation is high enough to provide functionality of one disassembly machinery in the other organism. Some of these have come to the conclusion that single components of the machineries seem to be interchangeable [20], but others have come to apparently contradictory results $[28,49]$

The homologues of $\alpha$ SNAP and NSF in yeast are Sec17 and Sec18, respectively. The structures of Sec17 as well as the Sec18 N-terminal domain have been solved crystallographically $[30,55]$. In analogy to the neuronal SNARE complex, in vitro binding studies of Sec17 yeast complex coupled to GST-beads revealed that the stoichiometry of Sec17 to SNARE complex is 3:1 [53]. The crystal structures of the yeast and the neuronal SNARE complex as well as of NSF and the Sec18 Nterminal domains reveal a remarkable degree of similarity $[54,55,56,29]$, suggesting that the mode of interaction with their respective SNAP adapters might also be conserved. NSF and Sec18p may however not be directly interchangeable, because NSF is unable to complement the Sec18-1 mutation in yeast [49]. Nevertheless, 
functional homology between NSF and Sec18p has been proposed after it was shown that yeast cytosols can reconstitute NSF activity to NEM-treated mammalian in vitro transport assays $[57,80]$. Yeast cytosol yet might include additional factors to Sec18, so that the recovery here can not be reliably be ascribed to Sec18 function. When studying the interchangeability of the adapter proteins, yeast cytosol was unable to bind NSF to mammalian Golgi-membranes [28], but Sec17 could replace SNAP activity in mammalian intra-Golgi assays [49]. NSF efficiently bound to plastic-coupled Sec17 and Sec18 to $\alpha$ SNAP [20]. Some functional homology thus seems to exist, but to which extent and whether for all of the proteins involved in the mechanism, remains unclear. No time-resolved experiments have yet been made, which have directly assessed the influence of exchanging yeast and mammalian proteins during SNARE complex disassembly.

I hence disassembled yeast SNARE complexes using components of the mammalian machinery and vice versa. Additionally, I used various combinations of the yeast and the mammalian components, to determine which of the parts or protein interfaces are especially conserved and whether the mechanisms found for the neuronal SNARE complex (as e.g. the 'membrane boost') are conserved in yeast.

A quantitative comparison of the actual complex turnover by the different combinations of disassembly machineries could not be made, because Sec18 as well as Sec17 turned out to be less stable under the purification conditions used than $\alpha$ SNAP and NSF. It soon became evident that both, Sec17 as well as Sec18, tend to loose activity very fast (in a day or two from purification). The experiments using the yeast machinery, therefore do not provide absolute activities or rates of disassembly. Nevertheless, qualitative interpretations can be made and some questions regarding the grade of conservation of the disassembly mechanism could be resolved merely on the basis of comparative measurements.

Target recognition is highly conserved. Strikingly, both the yeast and the disassembly machinery were able to disassemble both SNARE complexes (figures 4.49 and $4.47 \mathrm{~A}$ ). For both complexes, the yeast machinery disassembles less efficiently than the mammalian. Whether this is only due to a lower stability in the purification buffers or also has a physiological meaning remains unclear at this point.

Interestingly, both machineries dissociated their non-cognate complexes comparably fast and efficiently as their respective cognate complexes. This finding is in line with the hypothesis that rather the quaternary structure than the sequence conservation may be important for target recognition.

The adapter/enzyme interface is highly conserved. Disassembly of neuronal complexes by the yeast machinery (Sec17 and Sec18) and the combination of Sec18 and $\alpha$ SNAP worked equally efficient. The efficient disassembly mediated by $\alpha$ SNAP and Sec18 indicates that the interface and basic mechanism on the level of the adapter-enzyme interplay seem to be conserved (figure 4.47). In line with 
that proposal, Sec17 and NSF also efficiently disassembled the yeast complex, again pointing to a conservation of the adapter/enzyme interface.

Two non-cognate protein interactions strongly impair disassembly. Interestingly, the combination of Sec17 and NSF did not promote disassembly of the neuronal complex when used at the same concentrations as in the other combinations which all led to efficient disassembly (figure 4.47 B). Similarly, the combination of $\alpha$ SNAP and Sec18 did not disassemble the yeast complex under the same conditions used in the other experiments (figure $4.50 \mathrm{~B}$ ).

It can be excluded that the interfaces between Sec17 and NSF as well as between $\alpha$ SNAP and Sec18 have diverged so much during evolution, that enzyme and adapter cannot productively interact any longer, because these combined machineries should not be able to disassemble any SNARE complexes then. Both combinations however were able to promote disassembly of the respective other SNARE complex, the neuronal in the case of Sec18/ $\alpha$ SNAP and the yeast in the case of NSF/Sec17 as described in the last paragraph.

If one pictures the two connections between SNARE complex, SNAP adapter and enzyme, one notices that in both of these inefficient combinations both of the protein/protein interfaces are between non-cognate proteins, whereas all the other combinations discussed so far included only one non-cognate interaction interface. All these findings indicate that each of the protein/protein interfaces involved in the disassembly process is conserved enough to allow for exchange of proteins between yeast and mammals at one interface, but exchanges at two interfaces lead to synergistic defects of disassembly. The exchange of only the adapter protein, which brings about two non-cognate interfaces, therefore strongly impedes disassembly.

The membrane boost is conserved. The membrane boost observed for $\alpha \mathrm{SNAP}$ was found to be conserved for the yeast adapter protein Sec17. An amount of Sec17 and NSF, which did not at all disassemble SNARE-complexes in solution, did mediate disassembly on liposomes (4.48). It can thus be concluded that like for $\alpha$ SNAP, Sec17-efficacy is higher when the target complexes are incorporated into membranes. Similarly, the high efficiency of $\alpha$ SNAP when disassembling membranebound complexes can as well be observed for the disassembly of yeast complexes. Here, like for the neuronal complexes, $120 \mathrm{nM}$ of $\alpha$ SNAP suffice for efficient disassembly (see figure 4.49), when the yeast complex is incorporated into liposomes. This implies that the potentiation of $\alpha$ SNAP efficacy on membranes is independent of the neuronal SNARE complex as substrate.

Summary. It can be concluded that both of the protein interfaces between target, adapter and enzyme seem to be conserved well enough between yeast and mammals to allow proteins of the respective other organism to mediate disassembly. Notwithstanding the fact that disassembly occurs even with exchanged machineries, the kinetics of disassembly may be slightly impeded by two non-cognate proteins 
at any of the interaction interfaces, whereas they are strongly hampered when both interactions are mediated by non-cognate proteins. Additionally, the high $\alpha$ SNAP efficiency on membranes described earlier is independent of the nature of the SNARE target and the membrane boost conserved for the yeast adapter protein Sec17. 


\section{Chapter 6}

\subsection{Summary}

SNAREs (SNAP receptors) are small membrane-bound proteins which are key mediators of intracellular membrane fusion. The actual fusion step is believed to occur through complex formation of SNAREs sitting on opposing membranes, first bringing the membranes into close apposition and finally enabling them to fuse. SNARE complex spontaneously form upon mixing of the single SNAREs in solution and are extremely stable. After membrane merger, the complexed SNAREs need to be dissociated and recycled again to allow for further rounds of fusion. Since the dissociation of SNARE complexes requires energy, this so-called SNARE disassembly is mediated by an enzyme, the AAA ATPase NSF (N-ethyl maleimide sensitive fusion protein) and its cofactors $\alpha$ SNAP. Presumably three $\alpha$ SNAPs bind to one SNARE complex to form a binding site for the hexameric NSF. This then binds to the $\alpha$ SNAPs and catalyzes the reaction via hydrolysis of ATP.

The aim of this study was to investigate the molecular basis of SNARE disassembly including SNAP isoforms, putative regulatory factors and the degree of functional conservation. To this end, I established two fluorescence assays (based on FRET and fluorescence anisotropy) which allow for monitoring of the disassembly reaction in vitro. During the characterization of the disassembly reaction, it soon became evident that the recombinant $\alpha$ SNAP performed far less efficient when disassembling soluble SNARE complexes using these methods, than it did during disassembly of 'ex vivo' SNARE complexes using so-called plasma membrane sheets. The use of liposome-incorporated SNARE complex for disassembly sufficed to annihilate the observed discrepancy between the ex vivo and the in vitro preparation, indicating that the lipid membrane increases the potency of $\alpha$ SNAP during SNARE complex disassembly. I could further demonstrate, that an N-terminal deletion in the $\alpha$ SNAP molecule abolishes the membrane caused potentiation of efficacy observed for $\alpha$ SNAP. This finding suggests, that the N-terminus of $\alpha$ SNAP interacts with the membrane to increase the affinity or effectivity of $\alpha$ SNAP during disassembly. In addition to the long established interaction between $\alpha \mathrm{SNAP}$ and the membrane-bound SNARE complex, the membrane thus serves as a second SNAP receptor which increases $\alpha$ SNAP efficiency. Additionaly I demonstrated that the potentiation of SNAP efficiency in the presence of membranes is conserved in the 
SNAP isoform $\beta$ SNAP and in yeast.

Furthermore I performed disassembly reactions using different conditions, mutations and putative inhibitory proteins in order to gain molecular insights into the protein/protein interactions involved and putative regulatory mechanisms of SNARE disassembly. An antibody against the NSF N-terminal domain raised in the scope of this study eliminated SNARE disassembly completely and might serve as a useful tool to specifically block NSF action in in vivo experiments in the future. Three anti SNARE complex antibodies blocked disassembly to a certain extent but a complete block was possible only when two antibodies, one recognizing the $\mathrm{N}$ terminal end and the other recognizing the C-terminal end, were used in concert. Similarly, other SNARE complex mutants only abrogated SNARE disassembly when two alterations were introduced at non-proximal sites. These data indicate that the disassembly process is very robust and the disassembly machinery does not have a preference for either end of the complex.

The SNARE-complex interacting protein Complexin1 significantly inhibited SNAREcomplex disassembly in the fluorescence assays in an $\alpha$ SNAP concentration-dependent manner. This indicates that Complexin and $\alpha$ SNAP compete with each other for SNARE complex binding under in vitro conditions. The inhibition of disassembly by Complexin was more pronounced in solution than on liposomes, suggesting that Complexin affinity to the SNARE complex in the presence of membranes is not increased to the same extent as witnessed for $\alpha$ SNAP. A phosphorylation mutant of NSF, which has previously been suggested to have a reduced affinity to $\alpha$ SNAP, could not be definitely shown to exert a significant defect with regard to the disassembly reaction.

I further showed, that between yeast and mammals, both of the protein interfaces between target, adapter and enzyme seem to be conserved well enough to allow proteins of the respective other organism to mediate disassembly. Notwithstanding the fact that disassembly occurs even with exchanged machineries, the kinetics of disassembly may be slightly impeded by two non-cognate proteins at any of the interaction interfaces, whereas they are strongly hampered when both interactions are mediated by non-cognate proteins. Additionally, the high $\alpha$ SNAP efficiency on membranes described earlier is independent of the nature of the SNARE target and the membrane boost conserved for the yeast adapter protein Sec17. 


\subsection{Zusammenfassung}

Für die Fusion intrazellulärer biologischer Membranen sind Vertreter der konservierten Familie der SNARE-Proteine von essentieller Bedeutung. Man vermutet, dass gerichtete Komplexbildung zwischen SNAREs auf gegenüberliegenden Membranen dazu führt, dass die zu fusionierenden Mebranen in räumliche Nähe gezogen werden und schließlich verschmelzen. SNARE-Komplexe bilden sich spontan, wenn die einzelnen SNAREKomponenten in Lösung vermengt werden und sind äußerst stabil. Es muss daher Energie aufgewendet werden, um die SNARE-Komplexe nach der Membranfusion wieder in ihre Einzelteile zu dissoziieren um damit die einzelnen SNAREs für weitere Fusionsereignisse zur Verfügung zu stellen. Deshalb wird die Dissoziierung der SNARE-Komplexe durch ein Enzym, die AAA ATPase NSF sowie ihren Kofaktor $\alpha$ SNAP, vermittelt. Dazu binden vermutlich drei $\alpha$ SNAP-Moleküle einen SNARE-Komplex und bilden so ein Podest, das als Angriffsstelle für das hexamere, ringförmige NSF dient.

Das Ziel der vorliegenden Arbeit war es, die molekularen Grundlagen der SNAREKomplex-Dissoziierung inklusive der SNAP-Isoform $\beta$ SNAP und möglicher regulatorischer Faktoren, sowie den Grad der funktionellen Koservierung zu untersuchen. Hierzu wurden zwei fluoreszenzbasierte in vitro Methoden (FRET und FluoreszenzAnisotropy) zur direkten Messung des SNARE-Komplex Dissoziation durch NSF und $\alpha$ SNAP etabliert. Während der Charakterisierung der Reaktion zeigte sich, dass das verwendete rekombinante $\alpha$ SNAP deutlich effizienter auf einer Plasmamembranpräparation 'ex vivo' als in den FRET- und Anisotropie- in vitro-Ansätzen agierte. Es reichte aus, die rekombinanten SNARE-Komplexe vor der Reaktion in Liposomen zu inkorporieren, um die beobachtete Diskrepanz zwischen der ex vivo- und der in vitro-Präparation zu eliminieren. Dieses deutet darauf hin, dass Lipidmembranen die Fähigkeit von $\alpha$ SNAP, im Verbund mit NSF SNARE-Komplexe zu dissoziieren, erhöhen.

Weiter konnte ich den Unterschied zwischen der Effizienz des $\alpha$ SNAPs in Lösung und auf Liposomen aufheben, indem ich N-terminale Deletion des $\alpha$ SNAP-Proteins vornahm. Dieser Befund lässt darauf schließen, dass die N-terminale Interaktion von $\alpha$ SNAP mit der Membran dazu führt, dass sich die Affinität oder Effektivität von $\alpha$ SNAP während der SNARE-Komplex-Dissoziation steigert. Zusätzlich zu dem membrangebundenen SNARE-Komplex scheint die Membran selbst demnach als zweiter, kooperativer SNAP-Bindungspartner zu fungieren. Zudem konnte ich zeigen, dass die Verstärkung der SNAP-Effizienz in Gegenwart von Membranen auch für die Isoform $\beta$ SNAP und das Hefe-Homolog Sec17 konserviert ist.

Außerdem habe ich mithilfe verschiedener Mutanten und Inhibitoren mögliche regulatorische Angriffspunkte charakterisiert. Ein Antikörper gegen die N-terminal Domäne von NSF, der auch im Rahmen dieser Arbeit hergestellt wurde, vermochte die Dissoziationsreaktion komplett zu blockieren und könnte zukünftig für in vivoExperimente eingesetzt werden. Drei Antikörper gegen den SNARE-Komplex hemmten die Dissoziationsreaktion teilweise, aber eine komplette Inhibition gelang nur, wenn zwei Antikörper gemeinsam eingesetzt wurden, einer gegen den N-Terminus und 
der andere gegen den C-Terminus von $\alpha$ SNAP. Analog dazu blockierten SNAREKomplex-Mutanten die Dissoziationsreaktion nur, wenn zwei entfernte Positionen mutiert worden waren. All dies lässt darauf schließ, dass die Reaktion sehr robust ist.

Das SNARE-Komplex-bindende Protein Complexin1 inhibierte die Reaktion in Abhängigkeit von der $\alpha$ SNAP-Konzentration. Die Ergebnisse zeigen, dass Complexin und $\alpha$ SNAP unter in vitro-Bedingungen miteinander um die SNARE-KomplexBindung konkurrieren. Der inhibitorische Effekt von Complexin war in Lösung stärker als auf Liposomen, sodass davon ausgegangen werden kann, dass die ComplexinAffinität zum SNARE-Komplex sich in Gegenwart von Membranen nicht im selben Maße steigert wie die von $\alpha$ SNAP.

Schließlich konnte ich zeigte, dass beide Protein-Schnittstellen zwischen Substrat, Adaptor und Enzym zwischen Hefen und Säugern stark genug konserviert sind um den Austausch einzelner Komponenten zu ermöglichen, ohne dass die Reaktion zum Erliegen kommt. Dabei führt der Austausch des Adaptors zu einem weitaus stärkeren Defekt als der Austausch des Enzyms oder des Komplexes. Offenbar wird die Reaktion demnach nur dann schwer gestört, wenn sich an beiden Schnittstellen Proteine aus verschiedenen Organismen treffen. 


\section{Bibliography}

[1] Glick Bonifacino JS. The mechanisms of vesicle budding and fusion. Cell, 116(2):153-66, Jan 2004.

[2] J Faix, M Steinmetz, H Boves, R Kammerer, F Lottspeich, U Mintert, J Murphy, A Stock, U Aebi, and G Gerisch. Cortexillins, major determinants of cell shape and size, are actin-bundling proteins with a parallel coiled-coil tail. Cell, 86(4):631-42, Aug 1996. 0092-8674 Journal Article.

[3] D Fasshauer, D Bruns, B Shen, R Jahn, and A.T Brünger. A structural change occurs upon binding of syntaxin to snap-25. J. Biol. Chem., 7:4582-4590, 1997.

[4] D Fasshauer, H Otto, W Eliason, R Jahn, and A Brunger. Structural changes are associated with soluble n-ethylmaleimide- sensitive fusion protein attachment protein receptor complex formation. J. Biol. Chem., 272(44):28036-28041, 1997.

[5] T Weber, B Zemelman, J McNew, B Westermann, M Gmachl, F Parlati, T Sollner, and J Rothman. Snarepins: minimal machinery for membrane fusion. Cell, 92(6):759-772, 1998.

[6] D Fasshauer, R Sutton, A Brunger, and R Jahn. Conserved structural features of the synaptic fusion complex: Snare proteins reclassified as q- and r-snares. Proc. Natl. Acad. Sci. U S A, 95(26):15781-15786, 1998.

[7] K Fiebig, L Rice, E Pollock, and A Brunger. Folding intermediates of snare complex assembly. Nature Struct. Biol., 6(2):117-123, 1999.

[8] P I Hanson, R Roth, H Morisaki, R Jahn, and J E Heuser. Structure and conformational changes in nsf and its membrane receptor complexes visualized by quick-freeze/deep-etch electron microscopy. Cell, 90(3):523-35, Aug 1997.

[9] J Hay and R Scheller. Snares and nsf in targeted membrane fusion. Curr Opin Cell Biol, 9(4):505-12, 1997.

[10] J Hazzard, T Sudhof, and J Rizo. Nmr analysis of the structure of synaptobrevin and of its interaction with syntaxin. J. Biomol. NMR, 14(3):203-207, 1999. 
[11] M Margittai, D Fasshauer, S Pabst, R Jahn, and R Langen. Homo- and heterooligomeric snare complexes studied by site-directed spin labeling. J. Biol. Chem., 276(16):13169-77., 2001.

[12] D Fasshauer, W Antonin, V Subramaniam, and R Jahn. Snare assembly and disassembly exhibit a pronounced hysteresis. Nat Struct Biol, 9(2):144-51, Feb 2002. 1072-8368 Journal Article.

[13] H Otto, P.I Hanson, and R Jahn. Assembly and disassembly of a ternary complex of synaptobrevin, syntaxin, and snap-25 in the membrane of synaptic vesicles. Proc Natl Acad Sci USA, 1997.

[14] J Rothman. Mechanisms of intracellular protein transport. Nature, 372(6501):55-63, 1994.

[15] W Antonin, D Fasshauer, S Becker, R Jahn, and T Schneider. Crystal structure of the endosomal snare complex reveals common structural principles of all snares. Nat Struct Biol, 9(2):107-11, Feb 2002. 1072-8368 Journal Article.

[16] W Antonin, C Holroyd, D Fasshauer, S Pabst, G Von Mollard, and R Jahn. A snare complex mediating fusion of late endosomes defines conserved properties of snare structure and function. EMBO J., 19(23):6453-6464, 2000.

[17] D Zwilling, A Cypionka, W Pohl, D Fasshauer, P Walla, M Wahl, and R Jahn. Early endosomal snares form a structurally conserved snare complex and fuse liposomes with multiple topologies. Embo J, 26(1):9-18, Jan 2007.

[18] S Hwang and J Lee. Neuron cell type-specific snap-25 expression driven by multiple regulatory elements in the nematode caenorhabditis elegans. $J \mathrm{Mol}$ Biol, 333(2):237-47, Oct 2003. 0022-2836 Journal Article.

[19] R Jahn and T.C Südhof. Membrane fusion and exocytosis. Annu. Rev. Biochem., 68:863-911, 1999.

[20] G Steel, A Laude, A Boojawan, D Harvey, and A Morgan. Biochemical analysis of the saccharomyces cerevisiae sec18 gene product: implications for the molecular mechanism of membrane fusion. Biochemistry, 38(24):7764-72, 1999.

[21] S Whiteheart, K Rossnagel, S Buhrow, M Brunner, R Jaenicke, and J Rothman. N-ethylmaleimide-sensitive fusion protein: a trimeric atpase whose hydrolysis of atp is required for membrane fusion. Journal of Cell Biology, 126(4):945-54, 1994.

[22] E Nagiec, A Bernstein, and S Whiteheart. Each domain of the nethylmaleimide-sensitive fusion protein contributes to its transport activity. $J$ Biol Chem, 270(49):29182-8, 1995. 
[23] K Fleming, T Hohl, R Yu, S Muller, B Wolpensinger, A Engel, H Engelhardt, A Brunger, T Sollner, and P Hanson. A revised model for the oligomeric state of the n-ethylmaleimide- sensitive fusion protein, nsf. J Biol Chem, 273(25):1567581, 1998.

[24] D Wilson, S Whiteheart, M Wiedmann, M Brunner, and J Rothman. A multisubunit particle implicated in membrane fusion. Journal of Cell Biology, 117(3):531-8, 1992.

[25] EH Egelman. Bacterial helicases. J Struct Biol., 124(2-3):123-8, Dec 1998.

[26] Lupas Schmidt M. Structure and mechanism of atp-dependent proteases. Curr Opin Chem Biol., 3(5):584-91, 1999.

[27] Andreas Martin, Tania A Baker, and Robert T Sauer. Rebuilt aaa + motors reveal operating principles for atp-fuelled machines. Nature, 437(7062):1115-20, Oct 2005.

[28] D Clary, I Griff, and J Rothman. Snaps, a family of nsf attachment proteins involved in intracellular membrane fusion in animals and yeast. Cell, 61(4):70921, May 1990. 0092-8674 Journal Article.

[29] Eduard Bitto, Craig A Bingman, Dmitry A Kondrashov, Jason G McCoy, Ryan M Bannen, Gary E Wesenberg, and George N Phillips. Structure and dynamics of gamma-snap: insight into flexibility of proteins from the snap family. Proteins, 70(1):93-104, Jan 2008.

[30] L Rice and A Brunger. Crystal structure of the vesicular transport protein sec17: implications for snap function in snare complex disassembly. Mol Cell, 4(1):85-95, 1999.

[31] Xu Xu J. Differential regulation of exocytosis by alpha- and beta-snaps. $J$ Neurosci., 22(1):53-61, Jan 2002.

[32] G Stenbeck. Soluble nsf-attachment proteins. Int J Biochem Cell Biol., 30(5):573-7, May 1998.

[33] A W Sudlow, B W McFerran, H Bodill, R J Barnard, A Morgan, and R D Burgoyne. Similar effects of alpha- and beta-snap on ca $(2+)$-regulated exocytosis. FEBS Lett, 393(2-3):185-8, Sep 1996.

[34] S Whiteheart, I Griff, M Brunner, D Clary, T Mayer, S Buhrow, and J Rothman. Snap family of nsf attachment proteins includes a brain-specific isoform [see comments]. Nature, 362(6418):353-5, 1993.

[35] E Matveeva, S Whiteheart, T Vanaman, and J Slevin. Phosphorylation of the n-ethylmaleimide-sensitive factor is associated with depolarization-dependent neurotransmitter release from synaptosomes. J Biol Chem, 276(15):12174-81, Apr 2001. 0021-9258 Journal Article. 
[36] Huong Huynh, Nunzio Bottini, Scott Williams, Vera Cherepanov, Lucia Musumeci, Kan Saito, Shane Bruckner, Eric Vachon, Xiaodong Wang, Joshua Kruger, Chung-Wai Chow, Maurizio Pellecchia, Edvard Monosov, Peter A Greer, William Trimble, Gregory P Downey, and Tomas Mustelin. Control of vesicle fusion by a tyrosine phosphatase. Nat Cell Biol, 6(9):831-9, Sep 2004 .

[37] H Hirling and R H Scheller. Phosphorylation of synaptic vesicle proteins: modulation of the alpha snap interaction with the core complex. Proc Natl Acad Sci USA, 93(21):11945-9, Oct 1996.

[38] T Ishizuka, H Saisu, S Odani, and T Abe. Synaphin: a protein associated with the docking/fusion complex in presynaptic terminals. Biochem Biophys Res Commun, 213(3):1107-14, Aug 1995.

[39] H McMahon, M Missler, C Li, and T Sudhof. Complexins: cytosolic proteins that regulate snap receptor function. Cell, 83(1):111-9, 1995.

[40] S Pabst, J Hazzard, W Antonin, T Sudhof, R Jahn, J Rizo, and D Fasshauer. Selective interaction of complexin with the neuronal snare complex: determination of the binding regions. J Biol Chem, 275(26):19808-18, 2000.

[41] S Pabst, M Margittai, D Vainius, R Langen, R Jahn, and D Fasshauer. Rapid and selective binding to the synaptic snare complex suggests a modulatory role of complexins in neuroexocytosis. J Biol Chem, 277(10):7838-48, Mar 2002. 0021-9258 Journal Article.

[42] X Chen, D Tomchick, E Kovrigin, D Arac, M Machius, T Sudhof, and J Rizo. Three-dimensional structure of the complexin/snare complex. Neuron, 33(3):397-409, Jan 2002. 0896-6273 Journal Article.

[43] C Giraudo, W Eng, T Melia, and J Rothman. A clamping mechanism involved in snare-dependent exocytosis. Science, 313(5787):676-80, Aug 2006.

[44] Johanna R Schaub, Xiaobing Lu, Blair Doneske, Yeon-Kyun Shin, and James A McNew. Hemifusion arrest by complexin is relieved by ca2+-synaptotagmin i. Nat Struct Mol Biol, 13(8):748-50, Aug 2006.

[45] Jiong Tang, Anton Maximov, Ok-Ho Shin, Han Dai, Josep Rizo, and Thomas C Südhof. A complexin/synaptotagmin 1 switch controls fast synaptic vesicle exocytosis. Cell, 126(6):1175-87, Sep 2006.

[46] K Hu, J Carroll, C Rickman, and B Davletov. Action of complexin on snare complex. J Biol Chem, Aug 2002. 0021-9258 Journal article.

[47] TJ Siddiqui, O Vites, A Stein, R Heintzmann, R Jahn, and D Fasshauer. Determinants of synaptobrevin regulation in membranes. Mol Biol Cell., 18(6):203746, 2007. 
[48] Rong Guan, Han Dai, and Josep Rizo. Binding of the munc13-1 mun domain to membrane-anchored snare complexes. Biochemistry, 47(6):1474-81, Feb 2008.

[49] I Griff, R Schekman, J Rothman, and C Kaiser. The yeast sec17 gene product is functionally equivalent to mammalian alpha-snap protein. J. Biol. Chem., 267(17):12106-15, 1992.

[50] G Steel, G Buchheim, J Edwardson, and P Woodman. Evidence for interaction of the fusion protein alpha-snap with membrane lipid. Biochem J, 325( $\mathrm{Pt}$ 2):511-8, 1997.

[51] S Whiteheart, M Brunner, D Wilson, M Wiedmann, and J Rothman. Soluble n-ethylmaleimide-sensitive fusion attachment proteins (snaps) bind to a multisnap receptor complex in golgi membranes. J. Biol. Chem., 267(17):12239-43, 1992.

[52] Pavel Strop, Stephen E Kaiser, Marija Vrljic, and Axel T Brunger. The structure of the yeast plasma membrane snare complex reveals destabilizing waterfilled cavities. J Biol Chem, 283(2):1113-9, Jan 2008.

[53] G Rossi, A Salminen, L Rice, A Brunger, and P Brennwald. Analysis of a yeast snare complex reveals remarkable similarity to the neuronal snare complex and a novel function for the c terminus of the snap-25 homolog, sec9. J Biol Chem, 272(26):16610-7, 1997.

[54] R Yu, R Jahn, and A Brunger. Nsf n-terminal domain crystal structure: models of nsf function. Mol Cell, 4(1):97-107, 1999.

[55] S M Babor and D Fass. Crystal structure of the sec18p n-terminal domain. Proc Natl Acad Sci USA, 96(26):14759-64, Dec 1999.

[56] A May, K Misura, S Whiteheart, and W Weis. Crystal structure of the aminoterminal domain of n-ethylmaleimide- sensitive fusion protein. Nat Cell Biol, 1(3):175-82, 1999.

[57] D Wilson, C Wilcox, G Flynn, E Chen, W Kuang, W Henzel, M Block, A Ullrich, and J Rothman. A fusion protein required for vesicle-mediated transport in both mammalian cells and yeast. Nature, 339(6223):355-9, 1989.

[58] P Woodman. The roles of nsf, snaps and snares during membrane fusion. Biochim Biophys Acta, 1357(2):155-72, 1997.

[59] U Laemmli. Cleavage of structural proteins during the assembly of the head of bacteriophage t4. Nature, 227(259):680-5, 1970.

[60] M Margittai, H Otto, and R Jahn. A stable interaction between syntaxin 1a and synaptobrevin 2 mediated by their transmembrane domains. FEBS Lett, 446:40-44, 1999. 
[61] C Wimmer, T Hohl, C Hughes, S Muller, T Sollner, A Engel, and J Rothman. Molecular mass, stoichiometry, and assembly of $20 \mathrm{~s}$ particles. J Biol Chem, 276(31):29091-7, Aug 2001. 0021-9258 Journal Article.

[62] Joshua M Lauer, Seema Dalal, Karla E Marz, Michael L Nonet, and Phyllis I Hanson. Snare complex zero layer residues are not critical for n-ethylmaleimidesensitive factor-mediated disassembly. J Biol Chem, 281(21):14823-32, May 2006 .

[63] D Fasshauer, W Eliason, A Brunger, and R Jahn. Identification of a minimal core of the synaptic snare complex sufficient for reversible assembly and disassembly. Biochemistry, 37(29):10354-10362, 1998.

[64] S Scales, B Yoo, and R Scheller. The ionic layer is required for efficient dissociation of the snare complex by alpha-snap and nsf. Proc Natl Acad Sci U S A, 98(25):14262-7, Dec 2001. 0027-8424 Journal Article.

[65] S Martinez-Arca, S Arold, R Rudge, F Laroche, and T Galli. A mutant impaired in snare complex dissociation identifies the plasma membrane as first target of synaptobrevin 2. Traffic, 5(5):371-82, May 2004. 1398-9219 Journal Article.

[66] S Whiteheart, T Schraw, and E Matveeva. N-ethylmaleimide sensitive factor (nsf) structure and function. Int Rev Cytol, 207:71-112, 2001. 0074-7696 Journal Article Review Review, Academic.

[67] K Marz, J Lauer, and P Hanson. Defining the snare complex binding surface of alpha -snap: implications for snare complex disassembly. J Biol Chem, Apr 2003. 0 1083-351x Journal article.

[68] V Malhotra, L Orci, B Glick, M Block, and J Rothman. Role of an nethylmaleimide-sensitive transport component in promoting fusion of transport vesicles with cisternae of the golgi stack. Cell, 54(2):221-7, Jul 1988. 0092-8674 Journal Article.

[69] T Sollner, S Whiteheart, M Brunner, H Erdjument-Bromage, S Geromanos, $\mathrm{P}$ Tempst, and J Rothman. Snap receptors implicated in vesicle targeting and fusion. Nature, 362(6418):318-24, 1993.

[70] T Hayashi, H McMahon, S Yamasaki, T Binz, Y Hata, T Sudhof, and H Niemann. Synaptic vesicle membrane fusion complex: action of clostridial neurotoxins on assembly. EMBO J., 13(21):5051-5061, 1994.

[71] T Hayashi, S Yamasaki, S Nauenburg, T Binz, and H Niemann. Disassembly of the reconstituted synaptic vesicle membrane fusion complex in vitro. EMBO J, 14(10):2317-25, May 1995. 
[72] M Gmachl and C Wimmer. Sequential involvement of p115, snares, and rab proteins in intra-golgi protein transport. J Biol Chem, 276(21):18178-84, May 2001. 0021-9258 Journal Article.

[73] A Morgan and R D Burgoyne. A role for soluble nsf attachment proteins (snaps) in regulated exocytosis in adrenal chromaffin cells. EMBO J, 14(2):232-9, Jan 1995.

[74] E A Matveeva, P He, and S W Whiteheart. N-ethylmaleimide-sensitive fusion protein contains high and low affinity atp-binding sites that are functionally distinct. J Biol Chem, 272(42):26413-8, Oct 1997.

[75] W DeBello, V O'Connor, T Dresbach, S Whiteheart, S Wang, F Schweizer, H Betz, J Rothman, and G Augustine. Snap-mediated protein-protein interactions essential for neurotransmitter release. Nature, 373(6515):626-30, 1995.

[76] A Bracher, J Kadlec, H Betz, and W Weissenhorn. X-ray structure of a neuronal complexin-snare complex from squid. J Biol Chem, 277(29):26517-23, Jul 2002. 0021-9258 Journal Article.

[77] Keith Weninger, Mark E Bowen, Ucheor B Choi, Steven Chu, and Axel T Brunger. Accessory proteins stabilize the acceptor complex for synaptobrevin, the 1:1 syntaxin/snap-25 complex. Structure, 16(2):308-20, Feb 2008.

[78] M Bowen, K Weninger, A Brunger, and S Chu. Single molecule observation of liposome - bilayer fusion thermally induced by snares. Biophys J, Sep 2004. 0006-3495 Journal article.

[79] M Bowen, K Weninger, J Ernst, S Chu, and A Brunger. Single-molecule studies of synaptotagmin and complexin binding to the snare complex. Biophys J, 89(1):690-702, Jul 2005. 0006-3495 Journal Article.

[80] P G Woodman, L Rodriguez, and C J Stirling. Functional conservation of cytosolic proteins required for endosomal vesicle fusion. Yeast, 12(12):1251-62, Sep 1996. 


\section{Chapter 7}

\section{Appendix}

\section{List of Publications}

Marcin Barszczewski, John J. Chua, Alexander Stein, Ulrike Winter, Rainer Heintzmann, Felipe E. Zilly, Dirk Fasshauer, Thorsten Lang, and Reinhard Jahn:

A Novel Site of Action for $\alpha$ SNAP in the SNARE Conformational Cycle Controlling Membrane Fusion Molecular Biology of the Cell, Vol. 19, 776784 , March 2008

Ulrike Winter and Dirk Fasshauer:

Mechanism of SNARE complex assembly and disassembly

Chapter in 'SNARE proteins', Landes Bioscience, in press

Dana Bar-On ${ }^{\star}$, Ulrike Winter ${ }^{\star}$, Esther Nachliel, Menachem Gutman, Uri Ashery, Dirk Fasshauer and Thorsten Lang:

Cortical membrane sheets An 'ex vivo' platform for imaging kinetics of plasmalemmal protein interactions

submitted.

^ These authors contributed equally 


\subsection{Abbreviations and Symbols}

\begin{tabular}{ll} 
aa & Amino acid \\
APS & Ammonium persulfate \\
ATP & Adenosine triphosphate \\
BSA & Bovine serum albumine \\
cmc & Critical micelle concentration \\
DOM & Dodecylmaltoside \\
DTT & Dithiothreitol \\
DNA & Deoxyribonucleic acid \\
E. coli & Escherichia coli \\
EDTA & Ethylendiaminetetraacetic acid \\
FRET & Fluorescence resonance energy transfer \\
GST & Glutathion-S-transferase \\
GTP & Guanosine triphosphate \\
IgG & Immunoglobulin G \\
IAA & Indole acryl acetic acid \\
IPTG & Isopropyl- $\beta$-D-thiogalactoside \\
kDa & Kilo Dalton \\
LB & Luria Bertani medium \\
mS & Milli Siemens \\
NEM & N-ethyl-maleimide \\
Ni-NTA & Nickel-nitrilo-triacetate \\
NSF & N-ethylmaleimide-sensitive factor \\
PAGE & Polyacrylamide gel electrophoresis \\
PBS & Phosphate buffered saline \\
PCR & Polymerase chain reaction \\
PC & Phosphatidylcholine \\
PE & Phosphatidylethanolamine \\
PMSF & Phenymethylsulfonylflouride \\
PS & Phosphatidylserine \\
pH & Negative logarithm of H ${ }^{+}$concentration \\
rpm & Revolutions per minute \\
\hline
\end{tabular}


RT Room temperature

S Svedberg

$\mathrm{Sb} \quad$ Synaptobrevin

Sx Syntaxin

SDS Sodium dodecyl sulfate

SEM Standard error of the mean

SNAP Soluble-NSF-attachment protein

SNAP25 Synaptosome-associated protein of $25 \mathrm{kDa}$

SNARE SNAP-receptor

TB Terrific broth

TEMED N,N,N',N'-tetramethylethylene diamine

TMD Transmembrane domain

Tris Trisand-aminomethane

$\mathrm{V} / \mathrm{v} \quad$ Volume/volume

$\mathrm{W} / \mathrm{v} \quad$ Weigth/volume 


\subsection{Eidesstattliche Erklärung}

I hereby declare that the Ph.D. thesis entitled 'Biophysical Characterization of

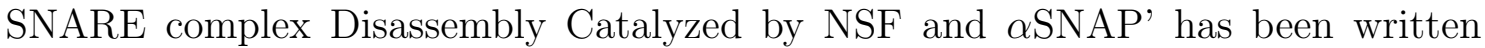
independently and with no other sources and aids than quoted.

Hiermit erkläre ich an Eides statt, dass ich die vorliegende Arbeit selbst und ohne weitere als die durch Zitate angegebene Hilfe angefertigt habe.

Ulrike Winter 


\section{Lebenslauf}

Name:

Geburtstag:

Geburtsort:

1985-1989:

1989-1998:

1995/96:

1998-2003:

September 2000-März 2001:

November 2003:

Januar-März 2004:

Seit April 2004:
Ulrike Winter

14.04.1979

Kiel

Grundschule Döhrnstrasse in Hamburg

Helene-Lange-Gymnasium in Hamburg

El Toro High School, Lake Forest, Kalifornien, USA

Studium der Biochemie an der Christian-AlbrechtsUniversität Kiel
Praktikum beim Laborjournal in Freiburg

Promotion am Max-Planck-Institut für biophysikalische Chemie, Göttingen, Abteilung Neurobiologie 


\subsection{Acknowledgements}

I am very grateful to Dr. Dirk Fasshauer for his excellent supervision and competent advice. In addition to creating a cooperative working athmosphere, he has always been very helpful and supportive in answering my questions and at the same time gave me so much freedom to try things on my own.

I would like to express my gratitude to Prof. Dr. Reinhard Jahn for being my thesis examiner as well as the opportunity to perform my $\mathrm{PhD}$ within the Dept. of Neurobiology and for providing a very stimulating working environment.

I would like to thank Prof. Dr. Ralf Ficner for being the co-referee of my thesis. I would like to thank Prof. Dr. Ralf Heinrich of the Dept. of Zoology, and Prof. Dr. Jörg Stülke of the Dept. of Molecular Microbiology, for being my oral examiners. I would additionally like to acknowledge the two further members of my thesis committee, Prof Dr. Robbert Gradstein and Prof Dr. Ivo Feussner.

Moreover, thank you very much to all the people who have been working with me and all those that have honoured me with their friendship and their presence. In particular, I would like to thank the colleagues who shared proteins, equipment and protocols with me and who helped me find my ways around the lab especially in the beginning.

Special thanks to Wolfgang Berning-Koch who often expressed and purified proteins for me. Furthermore, I would like to express my gratitude to Gottfried Mieskes, without whom the department would collapse. He always readily helps with technical problems of any kind. Thanks to Constanze Riel for careful proof-reading.

Thank you to Thorsten Lang and Dana Bar-On for collaboration on the membrane sheet experiments and Claudia Tomes for collaboration on phosphorylated NSF. Also, I would like to acknowledge my lab-rotation students Barbara Cokic, Hannes Uchtenhagen, Sebastian Gliem, Georg Raiser and Seong Joo Koo for working with me and sharing the excitement of the disassembly reaction.

I feel especially indebted to Georg Zeller for all his support and a wonderful year. Moreover I would like to thank him for critical reading of the manuscript and stimulating discussions.

Finally, I wish to say an enormous 'Dankeschön' to my family and my friends for constant support and encouragement throughout my $\mathrm{PhD}$ and previous studies. 